\title{
EXCITING MODELS
}

Exploring the applicability of human neuronal cell models for in vitro neurotoxicity screening and seizure liability assessment

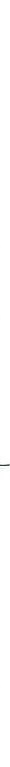




\section{EXCITING MODELS}

Exploring the Applicability of Human Neuronal Cell Models for In Vitro Neurotoxicity Screening and Seizure Liability Assessment

Anke Tukker 
Exciting models. Exploring the applicability of human neuronal cell models for in vitro neurotoxicity screening and seizure liability assessment.

Copyright (C) 2020 Anke Tukker

ISBN/EAN: 978-90-393-7267-8

Layout and printing: proefschrift-aio.nl

Cover design: Pelle van Braak

Illustrations: Bob Cooper

The research described in this thesis was performed at the Neurotoxicology Research Group, Toxicology Division, at the Institute for Risk Assessment Sciences (IRAS), Faculty of Veterinary Medicine, Utrecht University.

Printing of this thesis was financially supported by the Institute for Risk Assessment Sciences. 


\title{
EXCITING MODELS
}

\section{Exploring the Applicability of Human Neuronal Cell Models for In Vitro Neurotoxicity Screening and Seizure Liability Assessment}

\section{PRIKKELENDE MODELLEN}

Het Verkennen van de Toepasbaarheid van Humane Neuronale Celmodellen voor In Vitro Neurotoxicologisch Onderzoek en het Detecteren van Insults

(met een samenvatting in het Nederlands)

\section{Proefschrift}

\author{
ter verkrijging van de graad van doctor aan de \\ Universiteit Utrecht op gezag van de \\ rector magnificus, prof. dr. H.R.B.M. Kummeling, \\ ingevolge het besluit van het college voor promoties \\ in het openbaar te verdedigen op \\ dinsdag 6 oktober 2020 des avonds te 6.00 uur
}

door

Anke Marije Tukker

geboren op 9 november 1986

te Almere 


\section{Promotor:}

Prof. dr. ir. J. Legler

\section{Copromotor:}

Dr. R.H.S. Westerink

Dit proefschrift werd (mede) mogelijk gemaakt met financiële steun van het National Centre for the Replacement, Refinement and Reduction of Animals in Research (NC3Rs; projectnummer 50308-372160) en ZonMW (projectnummer 114027001: InnoSysTox). 
"If a solution fails to appear...

and yet we feel success is just around the corner, try resting for a while.

... Like the early morning frost, this intellectual refreshment withers the

parasitic and nasty vegetation that smothers the good seed. Bursting forth at last is the flower of truth."

\section{Santiago Ramón y Cajal,}

Advice for a Young Investigator, MIT press, p35 


\section{Table of contents}

CHAPTER 1

General Introduction

PART I

29

METHODS FOR IN VITRO NEUROTOXICITY TESTING AND SEIZURE

LIABILITTY ASSESSMENT

CHAPTER 2

31

Electrophysiological measurement of $\mathrm{GABA}_{\mathrm{A}}$ receptor modulation

CHAPTER 3

37

In vitro techniques for assessing neurotoxicity using human iPSC-derived neuronal models

PART II

SINGLE RECEPTOR TO FULL NETWORK

CHAPTER 4

Perfluorooctane sulfonate (PFOS) and perfluorooctanoate (PFOA) acutely affect human $\alpha_{1} \beta_{2} \gamma_{2 \mathrm{~L}} \mathrm{GABA}_{\mathrm{A}}$ receptor and spontaneous neuronal network function in vitro

PART III

HUMAN IPSC-DERIVED NEURONAL MODELS

CHAPTER 5

Is the time right for in vitro neurotoxicity testing using human iPSC-derived neurons?

CHAPTER 6

117

Human iPSC-derived neuronal models for in vitro neurotoxicity assessment 
HUMAN IPSC-DERIVED NEURONAL MODELS FOR IN VITRO SEIZURE

\section{LIABILITY ASSESSMENT}

\section{CHAPTER 7}

Towards animal-free neurotoxicity screening: applicability of hiPSC-derived neuronal models for in vitro seizure liability assessment

CHAPTER 8

Applicability of hiPSC-derived neuronal co-cultures and rodent primary cortical cultures for in vitro seizure liability assessment

PART V

SUMMARY, GENERAL DISCUSSION AND CONCLUSIONS

CHAPTER 9

Summary, general discussion and conclusions

APPENDICES

REFERENCES

NEDERLANDSE SAMENVATTING

ACKNOWLEDGEMENTS

ABOUT THE AUTHOR 


\section{Abbreviations}

\begin{tabular}{|c|c|c|c|}
\hline 4-AP & 4-aminopyridine & FWHH & full width at half height \\
\hline \multirow[t]{2}{*}[\mathrm{Ca}^{2+}]{$_{\mathrm{i}}$} & intracellular calcium & GABA & gamma-aminobutyric acid \\
\hline & concentration & $\mathrm{GABA}_{\mathrm{A}}-\mathrm{R}$ & ionotropic GABA receptor type $A$ \\
\hline ACh & acetylcholine & GFAP & glial fibrillary acidic protein \\
\hline AM & acetoxymethyl & hESC & human embryonic stem cell \\
\hline AMPA & $\begin{array}{l}\alpha \text {-amino-3-hydroxyl-5-methyl- } \\
4 \text {-isoxazolepropionic acid }\end{array}$ & hiPSC & $\begin{array}{l}\text { human induced pluripotent } \\
\text { stem cell }\end{array}$ \\
\hline ANOVA & analysis of variance & $\mathrm{Hz}$ & Hertz \\
\hline AP & action potential & IBI & inter-burst-interval \\
\hline $\mathrm{Ca}^{2+}$ & calcium & $\mathrm{IC}_{50}$ & effective concentration \\
\hline cDNA & complementary DNA & & inhibiting $50 \%$ of maximum \\
\hline CFDA & 5-carboxyfluorescein diacetate & & response \\
\hline CI & confidence interval & ICH & $\begin{array}{l}\text { International Conference on } \\
\text { Harmonisation }\end{array}$ \\
\hline CNS & central nervous system & ISI & inter-spike interval \\
\hline $\mathrm{Cl}^{-}$ & chlorine ion & & notassium \\
\hline CoV & coefficient of variation & $\mathrm{K}_{\mathrm{v}}$ & potassium channel \\
\hline CPZ & chlorpromazine & LCIS & life cell imaging solution \\
\hline DAPI & 4',6-diamidino-2-phenylindole & LOEC & $\begin{array}{l}\text { lowest observed effect } \\
\text { concentration }\end{array}$ \\
\hline DIC & differential interface contrast & mACh & muscarinic acetylcholine \\
\hline DIV & days in vitro & MAP2 & micro-tubule-associated \\
\hline DMSO & dimethyl sulfoxide & & protein 2 \\
\hline $\mathbf{E C}_{20}$ & effective concentration evoking & MEA & micro-electrode array \\
\hline & $20 \%$ of maximum response & MeHg & methylmercury \\
\hline $\mathbf{E C}_{50}$ & $\begin{array}{l}\text { effective concentration evoking } \\
50 \% \text { of maximum response }\end{array}$ & MBR & mean burst rate \\
\hline$E_{100}$ & effective concentration evoking & MNBR & mean network burst rate \\
\hline & $100 \%$ of maximum response & MSR & mean spike rate \\
\hline EMA & European Medicine Agency & $\mathrm{mV}$ & millivolt \\
\hline Em & emission & mwMEA & multi-well micro-electrode array \\
\hline EtOH & ethanol & n.a. & not applicable \\
\hline Ex & excitation & nACh & nicotinic acetylcholine \\
\hline $\mathbf{F}_{340}$ & $340 \mathrm{~nm}$ excitation wavelength & nACh-R & nicotinic acetylcholine receptor \\
\hline $\mathbf{F}_{380}$ & $380 \mathrm{~nm}$ excitation wavelength & $\mathrm{Na}_{\mathrm{v}}$ & sodium channel \\
\hline FBS & foetal bovine serum & NBA & Neurobasal ${ }^{\oplus}-\mathrm{A}$ \\
\hline FDA & Food and Drug Administration & NMDA & N-methyl-D-aspartic acid \\
\hline
\end{tabular}




\begin{tabular}{|c|c|}
\hline NPC & neural progenitor cell \\
\hline n.s. & non-significant \\
\hline NT2 & NTERA-2 \\
\hline PBS & phosphate buffered saline \\
\hline PCA & principal component analysis \\
\hline PEI & polyethyleneimine \\
\hline PFA & paraformaldehyde \\
\hline PFAS & polyfluoroalkyl substances \\
\hline PFOS & perfluorooctane sulfonate \\
\hline PFOA & perfluorooctanoic acid \\
\hline PFS & perfect focus system \\
\hline PLL & poly-L-lysine \\
\hline PLO & poly-L-ornithine \\
\hline PND & post-natal day \\
\hline PTX & picrotoxin \\
\hline PTZ & pentylenetetrazol \\
\hline rms & root mean square \\
\hline ROI & region of interest \\
\hline RT & room temperature \\
\hline S100 $\beta$ & S100 calcium binding protein $\beta$ \\
\hline SD & standard deviation \\
\hline SEM & standard error of the mean \\
\hline TR & treatment ratio \\
\hline vGAT & vesicular GABA transporter \\
\hline VGCC & voltage-gated calcium channel \\
\hline vGLuT & vesicular glutamate transporter \\
\hline $\mathbf{V}_{\mathrm{m}}$ & membrane potential \\
\hline WHO & World Health Organization \\
\hline X-Rhod-1 & X-rhodamine-1 \\
\hline
\end{tabular}


Chapter 1

General Introduction 


\subsection{CURRENT NEUROTOXICITY TESTING AND SEIZURE LIABILITY ASSESSMENT}

All over the world people suffer from mental illnesses such as depression, anxiety, bipolar disorders, or schizophrenia. The World Health Organization (WHO) estimated that mental disorders affect one in four people, placing them amongst the leading causes of ill-health worldwide (World Health Organization, 2013). Mental illnesses have a great impact on the day to day life of patients. Therefore, people suffering from this type of diseases often require medication to keep their condition stable and to reduce the disease burden. Schizophrenia, depression, anxiety or bipolar disorders all affect the brain and originate in the brain. Another group of diseases that affect the brain are neurodegenerative diseases like Parkinson's, Huntington's or Alzheimer's disease. The prevalence of these diseases is increasing, partially due to the extension of human lifespan. Alzheimer's disease is one of the most prevalent neurodegenerative diseases. It was estimated that in 2015 almost 47 million people worldwide suffered from this disease and the number is expected to almost double every 20 years (Prince et al., 2015). The increasing number of patients suffering from mental illnesses and neurodegenerative disorders combined with the high disease burden translates into an increasing demand for drugs that target the brain and thus the central nervous system (CNS).

\subsubsection{Drug development and neurotoxicity testing}

The drug research and development process lasts around 15 years and is very costly (Roses, 2008). Out of the over 1000 compounds, 250 make it to the preclinical studies of which 5 enter clinical trials, hopefully resulting in 1 approved drug (Fig. 1). The International Conference on Harmonisation (ICH) has formulated guidance for the pharmaceutical industry that should be followed during pharmaceutical product development and later on while registering the drug for market access. Before drugs can enter the Western market, they need to meet the safety requirements set by regulatory authorities, the European Medicine Agency (EMA) for Europe and the US Food and Drug Administration (FDA) for the United States of America. These regulatory authorities and the ICH guidelines both rely heavily on animal studies in their requirements. One of the legal requirements for all drugs and thus CNS drugs is that they must pass neurotoxicity studies (ICH, 2001). In these studies, the potential of the drug to harm or interfere with the nervous system is investigated. Assessment of potential functional impairments such as learning and memory deficiency and behavioural changes is done using in vivo experiments with associated neuropathological endpoints. 


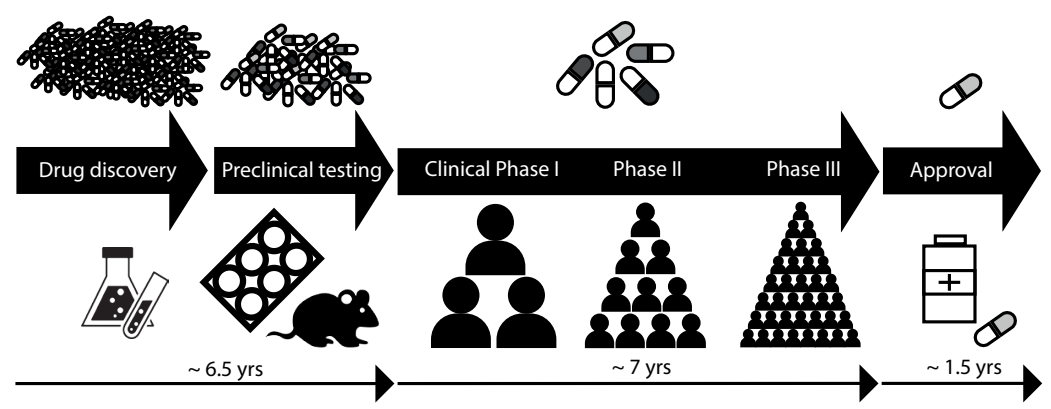

Figure 1. Schematic overview of the drug development pipeline. Drug discovery starts with over 1000 compounds. Entering the preclinical stage, about 250 compounds are left. Following in vitro and in vivo testing around 5 compounds are left. These compounds enter the clinical test phases. Ultimately, one compound will be registered on the market. The drug development pipeline can take up to 15 years.

\subsubsection{Seizure liability assessment}

In addition to neurotoxicity studies, potential new drugs must undergo seizure liability assessment. Drug-induced seizures and convulsions are the most frequently encountered CNS-related adverse drug reactions (Authier et al., 2016). Seizures are life threatening events in which there is a temporary dysfunction of the brain. They are characterised by periods of excessive synchronous neuronal discharge (Gotman, 2011). During a seizure, neurons fire abnormally and in a hyper-synchronised manner, exhibiting uncontrolled hyper-excitability (Easter et al., 2009; Jiruska et al., 2013). Especially drugs targeting the CNS are likely to cause seizures as an adverse drug reaction. The increased risk arises from the fact that the drug must enter the CNS to reach its target and has thus a higher brain penetration and higher affinity for nervous system targets (Gao et al., 2016). Seizure liability assessment is carried out late in the pre-clinical drug discovery process, often using low-throughput in vivo experiments (Easter et al., 2009). This means that once the potential of a compound to cause seizures is discovered, a large amount of time and money has already been invested, at the expense of many animals. Despite the severity of this adverse drug reaction, there are no official guidelines outlining the procedure that should be followed. However, a survey from the Safety Pharmacology Society held in the pharmaceutical industry showed that the ex vivo hippocampal brain slice assay is the most frequently used technique for in vitro seizure liability testing (Authier et al., 2016). Though this assay accurately mimics the in vivo organisation of the brain with active and intact 
networks (Grainger et al., 2018) and different cell types, the life span of slices is relatively short (Buskila et al., 2015) making it a low throughput assay. Also, this assay requires specific expertise and equipment. Preparation of the slices can result in cellular damage or metabolic changes. This problem can be overcome by using organotypic slice assays. These slices are derived from neonatal rodents and can be cultured in vitro for weeks (Lein et al., 2011). Organotypic slices resemble the in vivo structure containing most neuronal subtypes present in the brain retaining intrinsic properties of brain tissue. Due to the long culture duration, recovery from preparation insults is possible and necrotic cells and debris disappear over time (Lein et al., 2011). However, a downside of this model is that its derived from neonatal tissue leading to the concern that it may not resemble the adult brain. Another frequently used model system is the rodent primary cortical culture (Grainger et al., 2018). Similar to the brain slice assay, this model contains most of the components of the in vivo cortex. However, the structure of the brain is lost. A benefit of this model is that it certainly has a higher throughput than slice assays. A downside is that it can take several weeks before the culture reaches a mature state. However, several studies have shown that these cultures can efficiently be used to detect seizures in vitro (Kreir et al., 2018; Bradley and Strock, 2019). Thus, although a number of assays has been developed, their disadvantages currently prevent their wide use as in vitro alternatives.

\subsubsection{Challenge of inter-species translation}

The aforementioned assays all come with challenges related to inter-species translation, hampering full predictivity for human outcome. Rodents diverged from humans 60 million years ago, resulting in very different brain structures (Clowry et al., 2010). For example, rodents have far less developed prefrontal and temporal cortices, whereas these areas form the majority of the human cortex (Dolmetsch and Geschwind, 2011). Cortical neurons are involved in seizures (Martín-López et al., 2017) and play thus a crucial role in in vitro seizure liability assessment. It is known that neurons from different species, even closely related ones, exhibit different electrophysiological behaviour (Steffenhagen et al., 2011). The forthcoming problem of inter-species translation is reflected in the fact that despite all drug development guidelines and recommendations, potential drug candidates frequently fail due to CNS related adverse drug reactions not detected in preclinical studies (Arrowsmith and Miller, 2013). On top of that, $10 \%$ of the drug attrition rate is due to CNS related adverse drug reactions and safety concerns (Fung et al., 2001). This clearly indicates that current test strategies and models used for seizure liability assessment are not predictive enough. 
Additionally, studies with animals or animal tissue are costly, time consuming and not amenable for high-throughput testing as well as ethically debated. Thus, there is a clear need for improvement of pre-clinical test strategies given the growing demand for pharmaceuticals targeting the CNS, the lack of adequate high-throughput models with sufficient predictivity and ethical concerns related to animal testing.

\subsection{ALTERNATIVE IN VITRO MODELS}

Concerns around the predictivity and utility of animal models (in vivo and ex vivo) as well as associated animal welfare concerns have stimulated scientists to look for better and more predictive alternative test strategies. These strategies preferably make use of human cells to minimalize animal testing and to prevent the need for inter-species translation. Human pluripotent stem cells might provide a solution. There are two main types of pluripotent stem cells: human embryonic stem cells (hESCs) and human induced pluripotent stem cells (hiPSCs).

\subsubsection{Human embryonic stem cells}

ESCs, whether human or from other species, are derived from the inner cell mass of blastocyst-stage embryos (Martin, 1981; Thomson et al., 1998). In the case of humans, this corresponds with a 2-4 days old embryo. ESCs are pluripotent and can thus still differentiate into any somatic cell (Evans and Kaufman, 1981). Cells maintain a stable genetic background for a prolonged time when kept in vitro (Thomson et al., 1998). Because these cells maintain their replicative capacity, they are an unlimited source of specific cell types with the same genetic background. Thus, they hold great promise for research purposes. It has been shown that these cells in vitro can develop into neural progenitor cells (NPCs; Reubinoff et al., 2001) and further into different neuronal subtypes such as, for example, dopaminergic neurons (Perrier et al., 2004), interneurons (Maroof et al., 2013), and motor neurons (Li et al., 2005). The derived NPCs can also differentiate into supporting cells such as oligodendrocytes (Nistor et al., 2005) and astrocytes (Emdad et al., 2012). Even though these cells hold great promise for developmental studies and therapies, they are subject to considerable ethical, legal and social controversy due to their embryonic origin (Robertson, 2010). Controversy goes so far that funding for research with hESCs is uncertain and depends on politicians in charge (Owen-Smith et al., 2012), creating a huge drawback for usage of these 
cells and thus progress in this field. It also means that hESCs are not (yet) a suitable alternative for animal experiments.

\subsubsection{Human induced pluripotent stem cell-derived neuronal cultures}

The discovery that pluripotent stem cells could be generated from fibroblasts through the addition of reprogramming factors (Takahashi and Yamanaka, 2006) changed the stem cell field. It led to the introduction of hiPSC-derived cells that circumvent the legal and ethical issues surrounding hESCs. The benefit of hiPSCs is that they can be derived from adult fibroblasts via a skin biopsy, thus without any major invasive procedures and ethical debate. Human iPSCs exhibit the same characteristics as hESCs, including self-renewal and the potential to differentiate into any other somatic cell type (Robinton and Daley, 2012). Also, they retain the genotype of the original fibroblast, and thus any cell generated from these iPSCs share the same genetic background. This makes iPSCs an ideal disease model because they allow for the comparison between patients with a certain disease (and genetic mutation) and healthy individuals.

Through a combination of growth factors and cell culture conditions, hiPSCs can be differentiated into NPCs (Dolmetsch and Geschwind, 2011). From these NPCs, different neurons such as dopaminergic (Swistowski et al., 2010), glutamatergic (Zeng et al., 2010), cholinergic (Hu et al., 2016), serotonergic (Vadodaria et al., 2018) and GABAergic (Liu and Zhang, 2011) neurons can be derived (Fig. 2). They can also be differentiated towards supportive cells such as astrocytes (Emdad et al., 2012; Juopperi et al., 2012) and microglia (Abud et al., 2017).

Human iPSCs thus offer a very practical way of investigating development and function of living human neurons, while circumventing ethical debates and interspecies translation. However, even though this model seems promising, there are some pitfalls in the usage of these cells for high-throughput neurotoxicity screening that must be overcome. Key challenges include batch to batch variation (Schwartzentruber et al., 2017; Little et al., 2019) and reproducibility, as small changes in growth conditions can result in unwanted differentiation, and the time it takes to generate mature neurons is problematically long. Differentiation protocols can take up to months (Odawara et al., 2016; Little et al., 2019) making it a labour intensive and expensive process. Although efforts are made to reduce culture duration (Qi et al., 2017), the introduction of commercially available hiPSC-derived neurons may make these cells more attractive for efficient screening. 


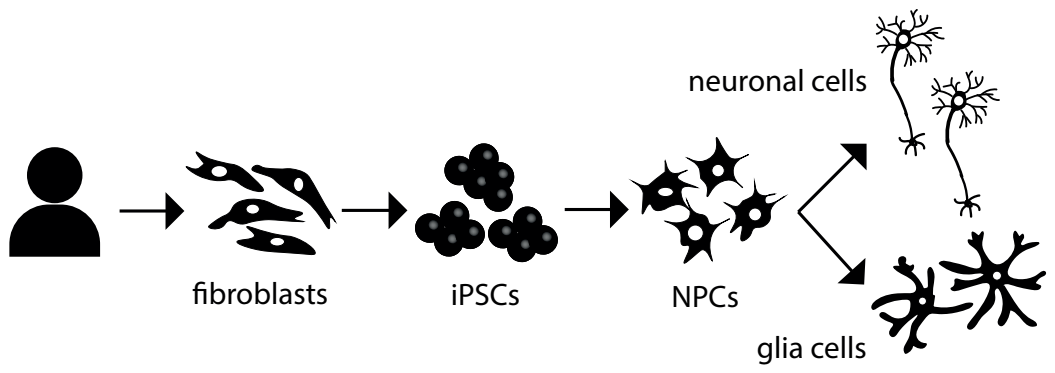

Figure 2. Schematic overview of the generation of hiPSC-derived neurons and glia cells. Fibroblasts are taken from individuals via a skin biopsy. With reprogramming factors, the cells are transformed into iPSCs. Growth factors and culture conditions will direct cells towards NPCs and finally towards neurons or glia cells.

Also, commercially available cells are produced in large quantities that are purity and quality controlled. Due to their recent introduction, only a small number of screens have been published (Richards et al., 2006; Xu et al., 2013; Sherman and Bang, 2018). However, a study showed that hits in a screen using human neuronal cells had different effects compared to rat primary cortical neurons (Richards et al., 2006), emphasising the difference between human and rodent models. These commercially available iPSCs may thus offer an ideal model for in vitro neurotoxicity testing and seizure liability assessment. The aim of this thesis is therefore to develop an alternative hiPSC-derived neuronal cell model that can be used for in vitro neurotoxicity testing and seizure liability assessment using commercially available cells.

\subsection{NEURONAL COMMUNICATION AND SEIZURES}

The first step in assessing the suitability of hiPSC-derived neurons for in vitro seizure liability assessment is to investigate whether these cells exhibit neurophysiological properties comparable to the human brain.

\subsubsection{Healthy neuronal function}

The nervous system is a sophisticated structure, which main function is to send, receive, integrate and interpret signals from all over the body. The brain forms the centre of this system. It consists of millions of neurons and supporting cells. In order to receive and send signals, neurons have two types of extensions to their 
cell body. On the one side there are numerous dendrites; on the other end, there is one axon. Together they are able to conduct and transmit signals via electrical impulses, also called action potentials. Dendrites are highly branched and receive signals, whereas the axon transmit the signal towards the synaptic terminal(s) from where the presynaptic neuron sends (chemical) signals to the postsynaptic (receiving) neuron (Fig. 3; left).

At membrane resting potential, sodium channels $\left(\mathrm{Na}_{v} \mathrm{~s}\right)$ are deactivated (closed). When a neuron receives an excitatory signal, it becomes activated and generates an action potential. During the generation of an action potential, $\mathrm{Na}_{v} \mathrm{~s}$ become active and open, thereby allowing an influx of sodium ions that cause an increase in membrane potential. This increase leads to a depolarisation. At some point, $\mathrm{Na}_{v} \mathrm{~s}$ close and potassium channels $\left(\mathrm{K}_{v} \mathrm{~s}\right)$ open, facilitating efflux of potassium ions. This brings the membrane to a state of repolarisation, followed by a hyperpolarisation in which ion levels are restored and the membrane is brought back to its resting potential. The action potential travels along the axon towards the presynaptic terminal. Once it reaches the synaptic terminal, voltage-gated calcium channels (VGCCs) open. These VGCCs are vital for neurotransmission since they trigger the neurotransmitter release machinery. Opening of VGCCs upon arrival of the action potential at the synaptic terminal, results in an influx of calcium ions $\left(\mathrm{Ca}^{2+}\right)$ through the presynaptic membrane as result of the intracellular and extracellular $\mathrm{Ca}^{2+}$ gradient. This results in an increase in intracellular $\mathrm{Ca}^{2+}$ concentration $\left(\left[\mathrm{Ca}^{2+}\right]_{\mathrm{i}}\right)$, triggering vesicles loaded with neurotransmitter to fuse with the presynaptic membrane (Neher and Sakaba, 2008). These vesicles thereby release neurotransmitter into the synaptic cleft. Neurotransmitters are endogenous chemicals that cause the conversion of the electrical signal of the action potential into a chemical signal. Following their release, neurotransmitters diffuse through the synaptic cleft and bind to neurotransmitter receptors on the postsynaptic neuron (Fig. 3; right). Depending on the type of receptor present, the chemical signal will be translated into a new action potential or it activates intracellular signalling pathways. However, a sufficient number of receptors must be activated to ensure signal transduction. Signal transduction is terminated via degradation or reuptake of the neurotransmitter from the synaptic cleft (Westerink, 2006). 


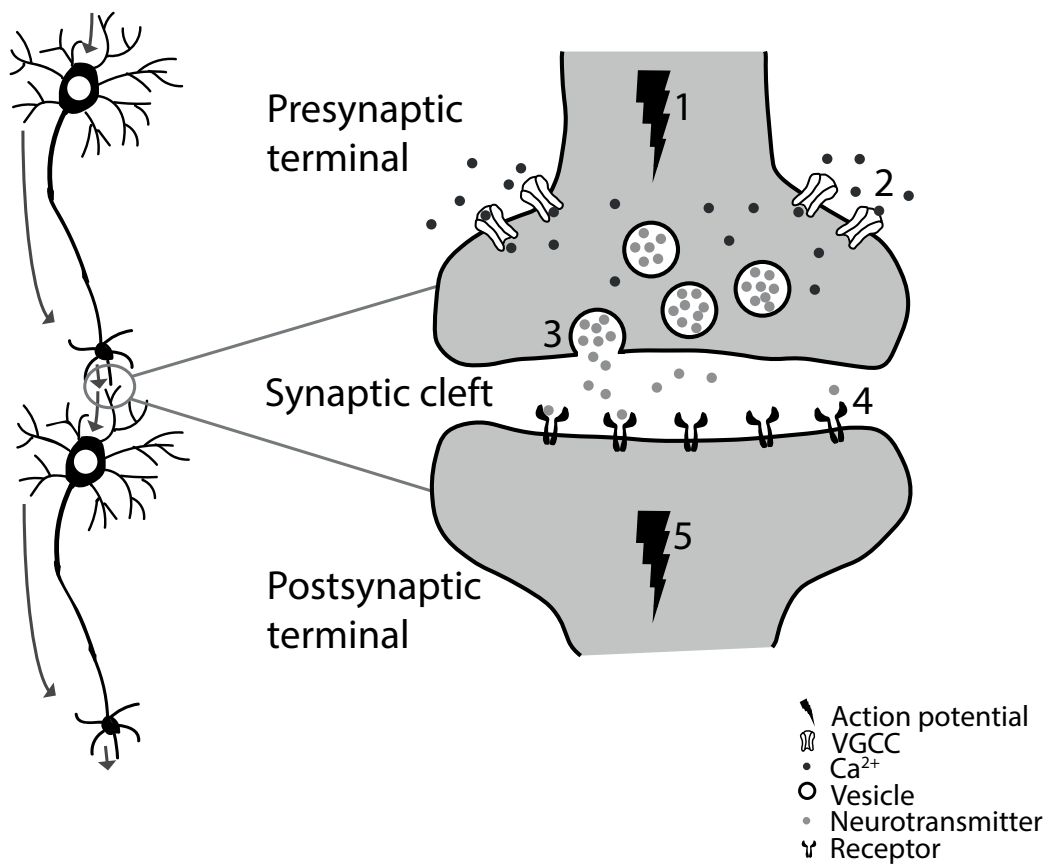

Figure 3. Schematic representation of neuronal signalling. Signals are received at dendrites and travel to the cell soma, where an action potential is generated. The action potential travels via the axon towards the presynaptic terminal, where the cell makes synaptic contact with the postsynaptic neuron (left side of the picture). Arrows indicate action potential travel direction. When an action potential reaches the presynaptic terminal (1; right side picture), VGCCs open allowing an increase of the $\left[\mathrm{Ca}^{2+}\right]_{\mathrm{i}}(2)$. This triggers the fusion of neurotransmitter containing vesicles with the cell membrane and neurotransmitters are released in the synaptic cleft (3). The released neurotransmitters migrate across the cleft to activate their corresponding receptor located at the postsynaptic membrane through binding (4). Depending on the receptor type, a new action potential is generated (5) or intracellular signalling pathways are activated.

\subsubsection{Neurotransmitter systems}

Activation of a neurotransmitter receptor by its corresponding neurotransmitter can result in neuronal excitation or inhibition, depending on the receptor type present. The different types of neurotransmitters and receptors systems are critically involved in a variety of processes such as anxiety, feeding behaviour, circadian rhythm, cognition, synaptic plasticity, learning and memory (D'Hulst et al., 2009; Picciotto et al., 2012; Meyerson et al., 2014; Chakraborty et al., 2019). 
The most abundant inhibitory neurotransmitter in the human cortex is gammaaminobutyric acid (GABA; Petroff, 2002; D'Hulst et al., 2009). Inhibitory GABAergic synapses contain either ligand-gated ion channel (ionotropic) $\mathrm{GABA}_{\mathrm{A}}$ receptors or G-protein-linked (metabotropic) $\mathrm{GABA}_{\mathrm{B}}$ receptors. When an agonist binds to the $\mathrm{GABA}_{\mathrm{A}}$ receptor, the receptor channel opens, resulting in an influx of chlorine ions $(\mathrm{Cl})$. In the mature brain, this creates a hyperpolarisation of the cell membrane, making it harder for the cell to reach an action potential, thereby inhibiting the neuron. In a developing brain, $\mathrm{GABA}_{\mathrm{A}}$ receptors have an excitatory function due to the reversed $\mathrm{Cl}^{-}$driving force in immature neurons (Ben-Ari, 2002). $\mathrm{GABA}_{\mathrm{A}}$ receptors consist of different combinations of $a, \beta, \gamma, \delta, \varepsilon, \theta, \pi$ and $\rho$ subunits. The $a_{1} \beta_{2} \gamma_{2 L}$ subunit combination is the most abundant and widely distributed in the brain (Olsen and Sieghart, 2008). GABA $A$ receptors have two GABA binding domains and several modulatory binding sites such as the benzodiazepine and picrotoxin site (D'Hulst et al., 2009; Carpenter et al., 2013). This is in contrast with the $\mathrm{GABA}_{B}$ receptor, which is a heterodimer composed of a B1 subunit that binds GABA and a B2 subunit that forms the G-protein coupling mechanism and contains a modulatory binding site (Pin and Prezeau, 2007). Binding of GABA to this receptor results in modulation of calcium and potassium channels.

Inhibition of the CNS can also occur via glycine that binds to the glycine receptor. This receptor is a ligand-gated chloride channel and belongs to the same super family of ion channels as the $\mathrm{GABA}_{\mathrm{A}}$ receptor (Cleland and Griggs, 2007).

The main excitatory neurotransmitter in the CNS is glutamate (Petroff, 2002). There are ionotropic and metabotropic glutamate receptors. Ionotropic glutamate receptors are ion channels mediating fast excitatory synaptic transmission. They consist of a combination of four subunits and can be divided in three groups: NMDA ( $\mathrm{N}$-methyl-D-aspartate), AMPA(a-amino-3-hydroxy-5-methyl-4-isoxazolepropionic acid) and kainate receptors (Meldrum, 2000). Binding of an agonist to either of the receptor types opens the channel creating a sodium or calcium influx (depending on the subtype) and potassium efflux (Traynelis et al., 2010). The channel of the NMDA receptor is blocked with a magnesium ion at resting membrane potential. Once a depolarisation occurs, the channel will open and magnesium is removed leading to calcium influx into the postsynaptic cell. However, NMDA receptors are also permeable to sodium and potassium. If the agonist binds to a metabotropic glutamate receptor, of which there are also three subtypes (mGluR group 1, mGluR group 2 and mGluR group 3), a slow synaptic response occurs and second messenger signalling pathways are activated (Niswender and Conn, 2010). 
Acetylcholine (ACh) is the main excitatory neurotransmitter in the peripheral nervous system and has a modulatory role in the CNS (Picciotto et al., 2012). The influence of the cholinergic system in the brain is neither purely excitatory nor inhibitory. Signals from the ionotropic nicotinic ACh (nACh) receptor are always excitatory, whereas signals from the metabotropic muscarinic ACh (mACh) receptor can be either excitatory or inhibitory.

Another neurotransmitter system is the dopaminergic system. This system receives input from the GABAergic system as well as the glutamatergic system. There are two classes of dopaminergic receptors, the D1-like (D1, D5) and the D2-like (D2, D3, D4), but they are all G-protein coupled receptors (Romanelli et al., 2010). Di activation is linked to regulation of $\mathrm{Na}_{v} \mathrm{~s}, \mathrm{VGCCs}$ and $\mathrm{K}_{\mathrm{v}} \mathrm{s}$, amongst other processes, and results in an increase of dopaminergic vesicle release (Neve et al., 2004). D2 receptors, also called auto-receptors, inhibit dopaminergic neurotransmission following activation (Neve et al., 2004). Dopaminergic neurons receive input from the glutamatergic and GABAergic system and depending on the input, dopaminergic neurotransmission increases or decreases.

In order to maintain normal brain function, the balance between these diverse excitatory and inhibitory signals must be precisely regulated. A schematic overview of the different neurotransmitter receptor types is given in figure 4. This figure also depicts whether they are ionotropic or metabotropic and, when applicable, inhibitory or excitatory. 


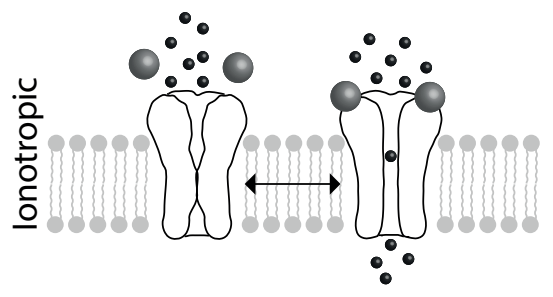

Inhibitory Excitatory

- $\mathrm{GABA}_{A}-\mathrm{R} \quad$-NMDA-R

- Glycine-R - AMPA-R

- Kainate-R

$\cdot \mathrm{nACh}-\mathrm{R}$

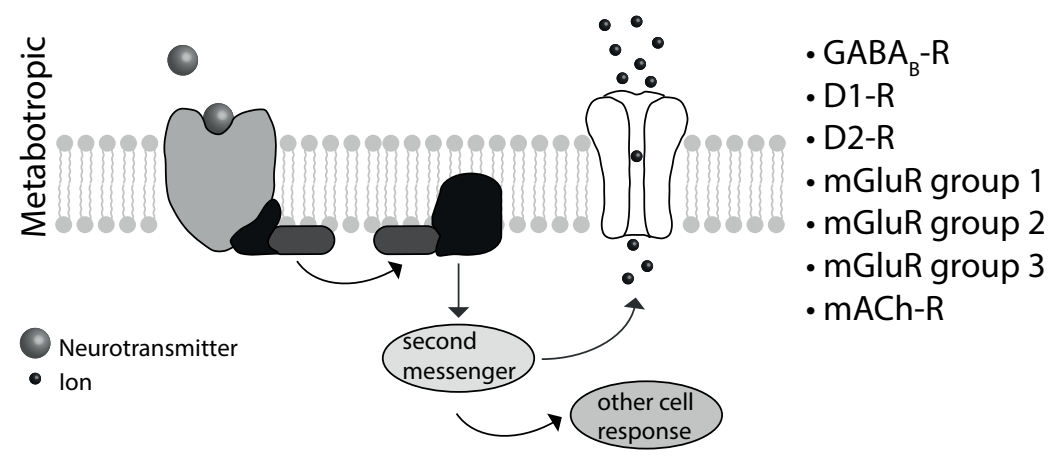

Figure 4. Schematic overview of the different neurotransmitter receptor types. Ionotropic receptors (top) undergo a confirmation change when their corresponding neurotransmitter binds. This allows for the passage of an ion through the channel. Inhibitory ionotropic receptors $(-R)$ are $G_{A B A}-R$, and glycine-R. Excitatory receptors are NMDA-R, AMPA-R, kainate- $R$ and $\mathrm{nACh}-\mathrm{R}$. Following binding of a neurotransmitter to a metabotropic receptor (bottom) a G-protein gets activated. The activated G-protein then activates an enzyme that either activates a second messenger system that triggers other cellular response or opens an ion channel. Metabotropic receptors are $\mathrm{GABA}_{\mathrm{B}}-\mathrm{R}$, D1-R, D2-R, mGluR group 1, mGluR group 2, mGluR group 3 and mACh-R.

\subsubsection{Physiology of seizures}

At the most elementary basis, seizures can be seen as a result of a disruption in the delicate balance between neuronal excitation and inhibition. It is thus logical that disruption of a mechanism that maintains the balance between inhibitory and excitatory activity of neurons is crucial for development of seizurogenic events (Lerche et al., 2001; Scharfman, 2007). Since GABA is the main inhibitory neurotransmitter in the brain and glutamate the main excitatory neurotransmitter, these systems play an important role in the development of seizures. 
Normally, cells have a resting membrane potential of $\sim-60 \mathrm{mV}$. This is the result of the balance between the intracellular potassium and extracellular sodium concentration. A perturbation in this balance can result in alterations in membrane potential, and thus alterations in the generation of action potentials and neuronal activity. Ion balance is normally maintained by pumps in the plasma membrane. Defective pumps could perturb the balance and increase the risk of seizures. Besides via pumps, extracellular ion concentrations are also controlled by astrocytes (Fellin and Haydon, 2005), which for example are involved in potassium clearance (Walz, 2000). Changes in sensitivity of potassium and sodium channels could also enhance neurotransmitter discharge. Also, changes in extracellular calcium are related to seizures (Antonio et al., 2016). Additionally, seizurogenic compounds can excite astrocytes (Fellin and Haydon, 2005) that then start to release glutamate, resulting in activation of the excitatory system.

An excessive discharge of neurotransmitters is not enough to cause a seizure. Synchronous firing must also occur. It has been shown that seizurogenic compounds create a paroxysmal depolarising shift: a network driven burst that enhances synchronisation (Johnston and Brown, 1984). Another way in which networks can be synchronised is via gap-junctions. They create a current flow from one cell to another (Scharfman, 2007). Via this current, neurons can synchronise rapidly.

Seizures thus cause changes in neuronal function occurring at the level of ion channels, ion homeostasis and network activity. Hence, functional analysis of hiPSC-derived neurons for seizure liability must be able to detect these changes.

\subsection{FUNCTIONAL IN VITRO SEIZURE LIABILITY ANALYSIS OF HIPSC-DERIVED NEURONAL CULTURES}

Ideally, an in vitro model captures the in vivo situation as closely as possible with sufficient complexity necessary to answer the research question, without overcomplicating the model (Westerink, 2013). For seizure liability assessment, it is important that cells form functional networks with a physiology mimicking the in vivo brain. In this case, the composition and ratio of various cell types present should resemble the brain regions involved in seizures. Targets for convulsants or seizurogenic compounds to act upon must be present and most importantly, the 
system must be able to display human seizure-like activity upon receiving triggers. As a starting point for testing the ability of a model to exhibit seizures, seizurogenic activity must occur following exposure to known seizurogenic compounds such as picrotoxin (PTX), 4-aminopyridine (4-AP) and strychnine. These compounds cause a dominating excitatory signal and induce a seizure-like discharge in neurons. This discharge in the neurons then forms hypersynchronous bursts in the in vitro network and is thought to be the main trigger for seizures (Ishii et al., 2017).

Seizures disrupt nervous system physiology, often in the absence of morphological changes. Therefore, seizure liability tests should be fast enough to detect action potentials and should assess ion channels, calcium changes or network responses. Several techniques exist and they are described in more detail in chapters 2 and 3.

Briefly, patch clamping techniques are accurate and efficient for measuring ionic currents (Sakmann and Neher, 1984) and can provide in depth information at receptor level. However, they are poorly amenable for high-throughput screens, technically demanding and invasive. Fluorescent imaging of changes in intracellular calcium levels or membrane potential has already a higher throughput and is less invasive. The technique enables visualisation of respectively free intracellular $\mathrm{Ca}^{2+}$ and membrane potential, and can be used to detect electrical and oscillatory activity, synchrony and even network activity (Smetters et al., 1999). Following exposure, changes in calcium or membrane potential can be assessed and the seizurogenic potential of the compound can be determined. Activity can also be monitored at network level. Micro-electrode arrays (MEA) allow for simultaneous extracellular recordings from multiple points within the network at the same time (Johnstone et al., 2010b). MEA measurements are non-invasive, and changes in activity following exposure can be measured in real-time.

Capturing and defining an in vitro seizure is complex due to the number of cellular mechanisms that can be involved. However, certain in vitro changes and observations can be indicative of in vivo seizures. An increased number of action potentials, thus an increased activity, can be seen as an increased spike frequency (Fig. 5). Increased activity is also reflected in increased bursting (Fig. 5) or increased calcium oscillations. Network synchronicity is reflected in synchronised calcium oscillations or in an increased network burst activity. Further indicators for seizurogenicity are increased (network) burst duration. 


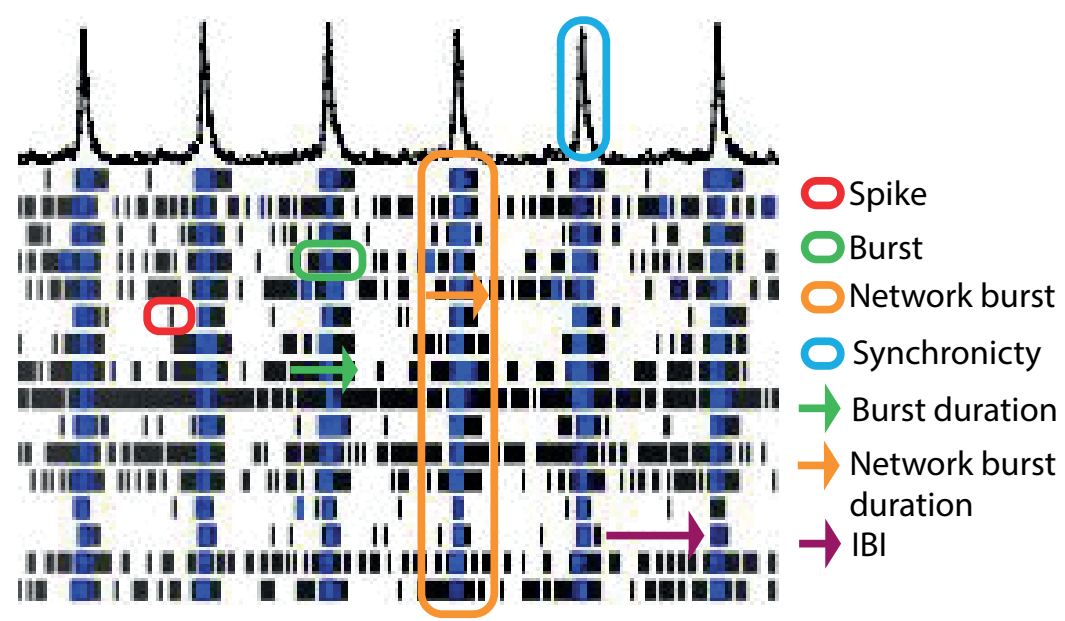

Figure 5. Raster plot illustrating significant MEA metric parameters. The picture depicts one well and each horizontal line represent one electrode and each tick mark (red circle) depicts one spike in a $\sim 100 \mathrm{~s}$ interval. An example of a burst is encircled in green and network burst in orange. Burst duration and network burst duration are depicted with a green and orange arrow respectively whereas an inter-burst-interval (IBI) is marked with a purple arrow. The cumulative trace above the raster plots indicates the synchronised activity between the different electrodes. The blue circle thus represents synchronicity. 


\subsection{THESIS OUTLINE}

The current test strategies for seizure liability assessment are not sufficient. They are not always predictive for outcomes in humans, often time consuming, not amenable for high throughput and/or ethically debated. Given the increasing number of people that depend on medication targeting the central nervous system, there is a clear need for improvement of test strategies. The research presented in this thesis aims at developing an alternative in vitro test system for seizure liability assessment using hiPSC-derived neurons. In this thesis, the main questions asked are:

- Does the model form a neuronal network resembling the in vivo complexity?

- Does the network become spontaneously active exhibiting synchronised bursting behaviour?

- Does the model exhibit seizure-like activity following exposure to a seizurogenic compound?

- How does the model compare to the current gold standard of rat primary cortical cultures?

This thesis consists of 5 parts. Part I outlines the different methods that were used. In Part II, a proof of concept of the methods used is given. Part III describes the different hiPSC-derived neuronal models that were tested and mainly focusses on questions 1 and 2. In Part IV, their suitability for seizure liability is assessed and data is compared with the current gold standard. This part of the thesis focusses on questions 3 and 4 . In the final part, Part V, the data presented in Part I-IV are summarised and discussed.

More specifically, detailed method descriptions can be found in Part I in which Chapter 2 explains the two-electrode voltage clamp technique in depth. Chapter 3 outlines different techniques that can be used to identify whether neuronal cells form spontaneously active networks that exhibit (network) bursting behaviour and how these techniques can be used for in vitro seizure liability assessment. Immunocytochemistry, calcium imaging and micro-electrode array recordings are discussed in more detail. 
Chapter 4, Part II, explores the potential of the techniques described in Part I for in vitro neurotoxicity testing, thereby linking single receptor studies to full network investigations. In order to do this, the effects of two environmental pollutants, perfluorooctane sulfonate (PFOS) and perfluorooctanoic acid (PFOA), on $\mathrm{GABA}_{\mathrm{A}}$ receptor function and neuronal network activity was investigated.

The next part of this thesis, Part III, moves away from methods and focusses on model development. In Chapter 5, the focus is mainly on mono-cultures. However, it is known that different types of neurons influence the behaviour of the culture. Also, astrocytes play an important role in the development and susceptibility of neurons. On top of that, to more closely resemble the in vivo brain, incorporation of astrocytes is a must. Therefore, the models become more complex in Chapter 6 . This chapter describes the development of different co-cultures and the effect of different ratios of glutamatergic to GABAergic neurons.

Whether the developed models are suitable for in vitro seizure liability assessment is outlined in Part IV. In Chapter 7, three different hiPSC-derived neuronal cocultures are exposed to the known seizurogenic compounds PTX, 4-AP and strychnine. The obtained data is compared with the current gold standard for MEA recordings, rat primary cortical cultures. One of these models is selected for further testing in Chapter 8 and exposed to known seizurogenic and nonseizurogenic compounds. Again, data is compared with data obtained from rat primary cortical cultures.

At the end of this thesis in Part $\mathbf{V}$, the findings of the presented work are summarised and placed in the context of the available literature in a general discussion in Chapter 9 . This is followed by conclusions and recommendations for future research. 


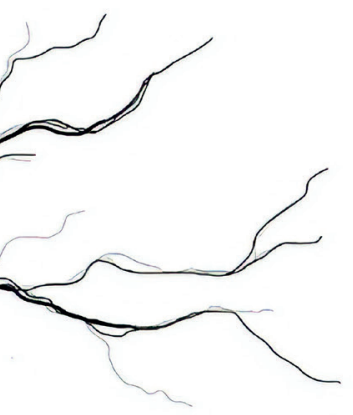




\section{METHODS FOR IN VITRO}

NEUROTOXICITY TESTING

AND SEIZURE LIABILITTY

ASSESSMENT 
Chapter 2

Electrophysiological measurement of $\mathrm{GABA}_{\mathrm{A}}$ receptor modulation 


\section{HUMAN GABA ${ }_{A}$ RECEPTORS}

Human $\mathrm{GABA}_{\mathrm{A}}$ receptors are ionotropic receptors that open upon binding of the neurotransmitter GABA or another agonist. GABA receptors are build up out of five subunits in various combinations of the different subunit types. Together, these subunits form an ion pore. The most common $\mathrm{GABA}_{\mathrm{A}}$ receptor is made up out of two $\alpha_{1}$, two $\beta_{2}$ and one $\gamma_{2}$ subunit (Fig. 1), forming the $\alpha_{1} \beta_{2} \gamma_{2}$ subtype (Sigel and Steinmann, 2012). Each such receptor contains two GABA binding sites on the $\alpha-\beta$ interface and several allosteric binding sites distributed over the receptor (D'Hulst et al., 2009). Example of an allosteric binding site is the benzodiazepine site located at the $\mathrm{a}-\mathrm{g}$ interface. Opening of the receptor's pore results in an influx of chloride ions and a hyperpolarisation of the cell membrane. Functional responses of the receptor can be measured with electrophysiological techniques, such as two-electrode voltage-clamp. The receptor can be expressed in oocytes of the South African clawed frog Xenopus laevis. The possibilities of this oocyte for experimental use were introduced in the 1950s (Brown, 2004).

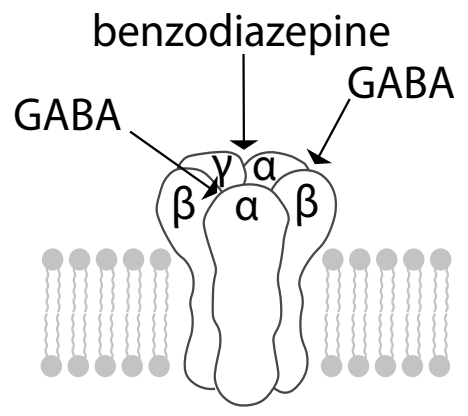

Figure 1. The $\mathrm{GABA}_{\mathrm{A}}$ receptor is inserted in the membrane and contains two GABA binding sites located at the interface of the $\alpha$ and $\beta$ subunits. The benzodiazepine binding site is located at the interface of the $\gamma$-a subunits.

\section{RECEPTOR EXPRESSION IN XENOPUS LAEVIS OOCTYES}

Oocytes isolated from the female Xenopus laevis frog can express functional foreign proteins on their membrane following microinjection of complementary DNA (cDNA) or mRNA in the oocyte's nucleus (Barnard et al., 1982). This cDNA or mRNA is transcribed and translated after which fully functional proteins are assembled and translocated to the cell membrane. This process is quite rapid, because a single oocyte has the same number of mitochondria and ribosomes as 
hundred thousand somatic cells and four times higher level of RNA polymerases (Brown, 2004). These oocytes are precursors for mature egg cells. Because they are normally deposited in harsh environments, they are resistant and equipped with all substrates needed for growth. Therefore, they do not need to take up anything from their surrounding environment. Oocytes thus express hardly any endogenous membrane transport proteins (Sigel and Minier, 2005), reducing background and making them an efficient model for electrophysiological recordings.

Xenopus laevis frogs are easy to keep in the laboratory. During a small surgical procedure, fully grown oocytes (stage V-VI oocytes) are isolated from adult female ovarian lobes. These oocytes are large cells (about $1.1-1.3 \mathrm{~mm}$ ), which makes them easy to handle. They are surrounded by a non-cellular fibrous vitelline layer and follicular cells. Only the follicular cells are removed by manual defolliculation and collagenase treatment. The vitelline layer provides stability during the experiment (Sigel and Minier, 2005). Once oocytes are clean, cDNA coding for the $\mathrm{GABA}_{\mathrm{A}}$ receptor with $\alpha_{1} \beta_{2} \gamma_{2}$ subunits can be injected into the nucleus. An incubation period of $4-6$ days at $21^{\circ} \mathrm{C}$ is required before the receptors are expressed at the oocyte's membrane (Fig. 2 upper part).

\section{TWO-ELECTRODE VOLTAGE-CLAMP TECHNIQUE}

With the two-electrode voltage-clamp technique it is possible to artificially control, or clamp, the membrane potential $\left(\mathrm{V}_{\mathrm{m}}\right)$ of large cells in order to study properties of electrogenic membrane receptors (Guan et al., 2013). Therefore, it allows for the identification of agonistic or antagonistic effects of compounds on a receptor. The voltage-clamp method was developed by Cole and Marmont. Later on, Hodgkin, Huxley and Katz modified it in the late 1940s to the twoelectrode voltage-clamp method for their giant axons of squid experiment (Hodgkin et al., 1952). This method can also be used on Xenopus laevis' oocytes.

Following the required incubation period, oocytes are placed in a custommade tube and injected with two glass micro-electrodes (Fig. 2 lower part). One electrode records the membrane potential and the other delivers the current (I). The membrane potential recording electrode connects to a feedback amplifier. The measured signal is then compared to the set potential, the voltage clamp command often set at $-60 \mathrm{mV}$. The differences between the measured value and the set potential is given as a current through the current delivering electrode across 
the membrane of the oocyte and to the bath-grounding electrode (Wagner et al., 2000). All changes in ion levels are now measured as compared to the baseline current. Oocytes that express a functioning $\mathrm{GABA}_{\mathrm{A}}$ receptor will exhibit an inward chloride current that creates a less negative membrane potential (hyperpolarisation) when superfused with GABA due to opening of the ion channel and $\mathrm{Cl}^{-}$influx. The voltage recording electrode now measures a change in membrane potential and a sufficient amount of current is injected to maintain the clamped potential of -60 $\mathrm{mV}$. The magnitude of injected current is proportional to the number of opened ion pores of the $\mathrm{GABA}_{\mathrm{A}}$ receptors. This technique thus provides a direct measurement of receptor functionality and can be used to assess the effect of external compounds on the receptor functioning.

Once it is confirmed that the oocyte expresses $\mathrm{GABA}_{\mathrm{A}}$ receptors, it can be exposed to the test compound dissolved in saline following a desensitisation period. From this exposure it will become clear whether the compound itself is a $\mathrm{GABA}_{\mathrm{A}}$ receptor agonist. If an inward current occurs, the compound is able to open the ion pore. Compounds can also work in combination with GABA. Therefore, following another desensitisation period, oocytes can be superfused with a mixture of GABA and different compound concentrations. 


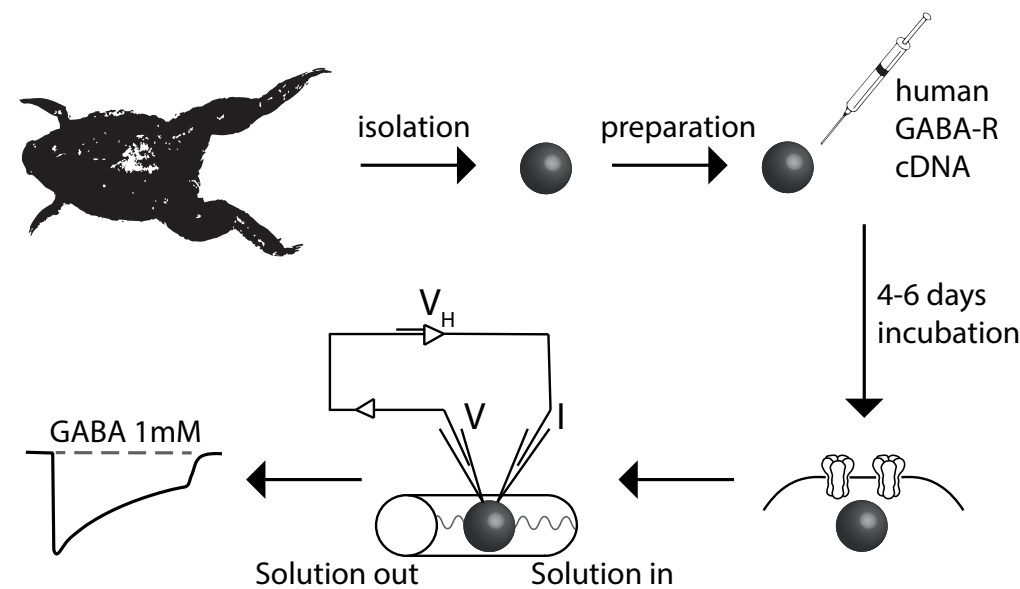

Figure 2. Oocytes are isolated from adult female Xenopus laevis frogs. Following preparation, they are injected with human cDNA coding for the different subunits of the $\mathrm{GABA}_{\mathrm{A}}$ receptor (GABA-R). Oocytes express the receptor on their membrane after $4-6$ days of incubation. Then, oocytes can be placed in a recording tube, where two micro-electrodes are injected into the oocyte and the membrane potential is clamped. Following superfusion with GABA, oocytes that express the GABA- $R$ will show an electrical response that is proportional to the number of activated $G_{A B A}$ receptors. 
Chapter 3

In vitro techniques for assessing neurotoxicity using human iPSC-derived neuronal models

Anke M. Tukker ${ }^{1}$, Fiona M.J. Wijnolts ${ }^{1}$, Aart de Groot ${ }^{1}$, Richard Wubbolts ${ }^{2}$, and Remco H.S. Westerink ${ }^{1}$

${ }^{1}$ Neurotoxicology Research Group, Toxicology and Pharmacology Division, Institute for Risk Assessment Sciences (IRAS),

${ }^{2}$ Centre for Cell Imaging (CCI), Department of Biochemistry and Cell Biology Faculty of Veterinary Medicine, Utrecht University, Utrecht, The Netherlands.

Chapter in: Aschner M., Costa L. (eds) Cell Culture Techniques. Neuromethods (2019), vol 145, pp. 17 - 35. Humana, New York, NY

doi:10.1007/978-1-4939-9228-7_2

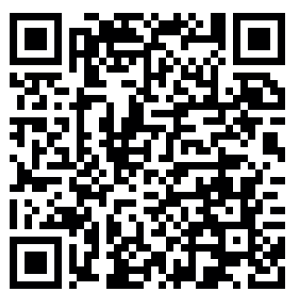




\section{ABSTRACT}

The central nervous system consists of a multitude of different neurons and supporting cells that form networks for transmitting neuronal signals. Proper function of the nervous system depends critically on a wide range of highly regulated processes including intracellular calcium homeostasis, neurotransmitter release and electrical activity. Due to the diversity of cell types and complexity of signalling processes, the (central) nervous system is very vulnerable to toxic insults.

Nowadays, a broad range of approaches and cell models is available to study neurotoxicity. In this chapter we show the applicability of human induced pluripotent stem cell (hiPSC)-derived neuronal co-cultures for in vitro neurotoxicity testing. We demonstrate that immunocytochemistry can be used to visualise networks of cultured cells and to differentiate between different cell types. Live cell imaging and electrophysiology techniques demonstrate that the neuronal networks develop spontaneous activity, including synchronized calcium oscillations that coincide with spontaneous changes in membrane potential as well as spontaneous electrical activity with defined (network) bursting. Importantly, as shown in this chapter, spontaneously active human iPSC-derived neuronal co-cultures are suitable for in vitro neurotoxicity assessment. Future application of live imaging and electrophysiological techniques on hiPSC from different donors and/or patients differentiated in different cell types holds great promise for personalized neurotoxicity assessment and safety screening.

Keywords: in vitro neurotoxicity screening, human induced pluripotent stem cell-derived neuronal models, single-cell fluorescent microscopy, calcium homeostasis, membrane potential, spontaneous neuronal activity, immunocytochemistry, multi-well microelectrode array 


\section{INTRODUCTION: NEURONAL NETWORK COMMUNICATION AND NEUROTOXICITY}

The (central) nervous system consists of sophisticated neuronal networks that controls body function, either via direct control or indirect via input in glands. The main function of neurons that make up the nervous system is to send and receive signals, a process called neurotransmission. To that aim, neurons have a typical structure with dendrites bringing the signal towards the cell body and an axon transmitting the signal away from the cell body. As soon as dendrites receive an excitatory chemical signal, the neuron becomes activated and translates this chemical input signal in an electrical signal, an action potential (AP). Via opening of voltage-gated sodium and potassium channels, the AP induces a change in membrane potential that travels via the cell body along the axon to the synapse at the axon terminal. There, the electrical signal (AP) is converted into a chemical signal that will be transferred to (an)other neuron(s). The first step in conversion from the electrical to the chemical signal involves opening of voltage-gated calcium channels (VGCC), resulting in a strong influx of calcium ions $\left(\mathrm{Ca}^{2+}\right)$. The resulting changes in the intracellular $\mathrm{Ca}^{2+}$ concentration $\left(\left[\mathrm{Ca}^{2+}\right]_{\mathrm{i}}\right)$ are involved in a variety of cellular processes such as excitability, plasticity, motility and viability (Westerink, 2006; Clapham, 2007). $\left[\mathrm{Ca}^{2+}\right]_{\mathrm{i}}$ is crucial for the regulation of neurotransmission as $\mathrm{Ca}^{2+}$ influx through VGCCs triggers the release of neurotransmitters from the presynaptic cell into the synaptic cleft (Barclay et al., 2005; Westerink, 2006; Südhof, 2014). Neurotransmitters are chemical signalling molecules that are stored in vesicles in the presynaptic neuron. There are different types of excitatory and inhibitory neurotransmitters, such as acetylcholine, dopamine, serotonin, glutamate and gamma-aminobutyric acid (GABA). After release into the synaptic cleft by fusion of the vesicles with the presynaptic plasma membrane, neurotransmitters can bind to receptors on the postsynaptic membrane. In the receiving cell, the signal can then be converted in a new AP or it can activate intracellular signalling pathways. The chemical signal is terminated by degradation or re-uptake of the neurotransmitters from the synaptic cleft (for review see (Westerink, 2006; Südhof, 2014).

Communication in neuronal networks thus critically depends on the structure of neurons, intact neuronal membranes and on regulation of cellular and molecular mechanisms underlying neurotransmission. Additionally, proper neuronal communication also depends on supporting cells such as oligodendrocytes, astrocytes and microglia. The multicellular nature of the networks can 
be confirmed with techniques as immunocytochemistry, whereas proper intracellular signalling can be studied with imaging techniques focusing on intracellular calcium levels and the membrane potential. Finally, the resulting network activity can be assessed with the use of multi-well micro-electrode arrays (mwMEA).

Its complexity and poor regenerative capacity make the nervous system vulnerable to toxic insults caused by chemical, physiological and biological agents that are present in the surrounding environment. Neurotoxicity is thus defined as an adverse effect caused by any of these agents on the structure and/or function of the nervous system. Nowadays, there is a broad range of approaches and cell models to study neurotoxicity in vitro.

\subsection{Methods to study neuronal network communication}

In vitro cell models should mimic the in vivo situation as closely as possible. For neurotoxicity testing this means that the in vitro model must form functional neuronal networks with both inhibitory and excitatory neurons as well as supporting cells. In order to circumvent interspecies translation, cells from human origin are the preferred option. Recently, human induced pluripotent stem cell (hiPSC)-derived neurons became commercially available. A benefit of these cells is that they do not require long differentiation into neural progenitor cells and ultimately into functional neurons, which can take several weeks (Görtz et al., 2004; Kuijlaars et al., 2016) till months (Odawara et al., 2016; Paavilainen et al., 2018). It has been shown that these hiPSC-derived neurons exhibit the behaviour and function of mature neurons (Odawara et al., 2016; Paavilainen et al., 2018). Therefore, we chose to use mixed hiPSC-derived neuronal models for the techniques described in this chapter.

First of all, it is important to determine whether the cultured cells form neuronal networks. This can be studied using fluorescent antibodies and confocal microscopy to detect specific target proteins in the cell or on the cell membrane. Besides studying network formation and complexity, immunofluorescent stainings can be used to differentiate between (neuronal) cell types in co-cultures.

Once the cells formed mixed neuronal networks, spontaneous network activity and the effect of toxic insults can be studied by looking at intracellular calcium homeostasis. Changes in $\left[\mathrm{Ca}^{2+}\right]_{i}$ can be analysed by loading the cultured cells with a high-affinity $\mathrm{Ca}^{2+}$-responsive fluorescent dye. Similarly, fluorescent 
voltage-sensitive dyes can be used to study changes in membrane potential as an indication for the occurrence of electrical activity.

Another way to look at spontaneous neuronal network activity and (network) bursting and toxic effects hereon is by the use of electrophysiological methods. The introduction of mwMEAs provided a way to grow cells on a culture surface with an integrated array of micro-electrodes. This allows for simultaneous and non-invasive recording of extracellular local field potentials at a millisecond time scale at different locations in the network grown in vitro (for review see (Johnstone et al., 2010)). Mammalian neuronal networks grown in vitro on mwMEA display many characteristics of in vivo neurons, including the development of spontaneous neuronal activity (Robinette et al., 2011) and synchronized bursting (Cotterill, Hall, et al., 2016). It has also been shown that these networks are responsive to neurotransmitters (Hondebrink et al., 2016), indicating the presence of a wide range of common neurotransmitter receptors. This technique offers consistent reproducibility across different laboratories (Novellino et al., 2011; Vassallo et al., 2017) and a high sensitivity and specificity (McConnell et al., 2012; Valdivia et al., 2014). For these reasons mwMEAs are seen as a suitable and consistent in vitro neurotoxicity screening method. Because measurements take place in a sterile environment, this technique allows for both acute (Nicolas et al., 2014; Hondebrink et al., 2017; Bradley et al., 2018) and chronic toxicity screening (Dingemans et al., 2016). Most mwMEA research was done with rat primary cortical cultures (Hogberg et al., 2011; Alloisio et al., 2015; Frank et al., 2017; Zwartsen et al., 2018), but recently it has been shown that also hiPSC co-cultures grow on mwMEA plates, develop spontaneous activity and bursting behaviour, and are suitable for neurotoxicity screening (Tukker et al., 2016, 2018; Odawara et al., 2018).

\section{MATERIALS}

\subsection{Cell culture}

For all techniques described in this chapter, we used commercially available hiPSC-derived neurons (iCell ${ }^{\circ}$ neurons and iCell $^{\circ}$ Glutaneurons) and astrocytes (iCell ${ }^{\circ}$ Astrocytes). All cells were obtained from Cellular Dynamics International (Madison, WI, USA). Cells were cultured at $37^{\circ} \mathrm{C}$ in a humidified $5 \% \mathrm{CO}_{2}$ incubator. From previous experiments, we know that these cells grow better on 
polyethyleneimine (PEI)-coated surfaces compared to poly-L-lysine (PLL)- or poly-L-ornithine (PLO)-coated materials. We therefore pre-coated all our cell culture surfaces with $0.1 \%$ PEI solution diluted in borate buffer $(24 \mathrm{mM}$ sodium borate/50 mM boric acid in Milli- $\mathrm{Q}$ pH adjusted to 8.4) unless stated otherwise. Co-cultures were grown in BrainPhys ${ }^{\mathrm{TM}}$ medium supplemented with $2 \%$ iCell Neuron supplement, $1 \%$ Nervous System supplement, $1 \%$ penicillin-streptomycin, $1 \%$ N2 supplement and $0.1 \%$ laminin (L2020 Sigma-Aldrich, Zwijndrecht, The Netherlands). Astrocytes (iCell ${ }^{\circ}$ ) were cultured in astrocyte medium (DMEM with high glucose and $10 \% \mathrm{FBS}, 1 \% \mathrm{~N} 2$ supplement and $1 \%$ penicillin-streptomycin).

Young astrocytes can proliferate rapidly and potentially overgrow neuronal cultures. Astrocytes were therefore passaged 2-3 times and stored in liquid nitrogen until use in co-culture with the hiPSC-derived neurons. Young astrocytes were thawed by gently swirling them for $2-3 \mathrm{~min}$ in a $37^{\circ} \mathrm{C}$ water bath. Then, the content of the vial was transferred to a sterile $50 \mathrm{~mL}$ tube. The vial was rinsed three times with astrocyte medium. Total volume in the $50 \mathrm{~mL}$ tube was brought to 10 $\mathrm{mL}$ and cells were centrifuged for $5 \mathrm{~min}$ at $1300 \mathrm{rpm}$. The cell pellet was dissolved in $6 \mathrm{~mL}$ astrocyte medium and cells were transferred to a $25 \mathrm{~cm}^{3} \mathrm{Geltrex}^{\mathrm{TM}}$-coated culture flask (Geltrex ${ }^{\mathrm{TM}}$ was added to cover the bottom of the flask and incubated for $45-60 \mathrm{~min}$ at $37^{\circ} \mathrm{C}$ in a humidified incubator, after which the Geltrex ${ }^{\mathrm{TM}}$ was removed). These flaks were used for a total of $1-1.4 \times 10^{6}$ cells. Astrocyte medium was replaced every 3-4 days. Cells were passaged by removing the medium, rinsing with PBS and adding $1 \mathrm{~mL} 0.0125 \%$ trypsin for $\sim 5 \mathrm{~min}$ to the flask. During trypsin incubation the flask was placed at $37^{\circ} \mathrm{C}$ in a humidified $5 \% \mathrm{CO}_{2}$ incubator. It is important to carefully check that cells are detached since astrocytes adhere strongly to the flask and may require more than $5 \mathrm{~min}$ incubation. Once the cells were detached, $9 \mathrm{~mL}$ of medium was added to the cells. Cells were counted and

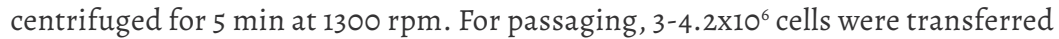
to a $75 \mathrm{~cm}^{3} \mathrm{Geltrex}^{\mathrm{TM}}$-coated culture flask and volume was brought to $20 \mathrm{~mL}$. In case cells were used for co-culturing and not for passaging, the cell pellet was dissolved in Complete iCell Neuronal Maintenance Medium supplemented with $1 \%$ penicillinstreptomycin, $2 \%$ iCell neuron medium supplement, $1 \%$ laminin (L2020, SigmaAldrich, Zwijndrecht, The Netherlands) into a 14.000 cells/ $\mu$ L solution.

Procedures for thawing of iCell ${ }^{\circ}$ neurons and iCell $^{\circ}$ Glutaneurons are comparable. The vial with neurons was thawed by gently swirling it for $2-3$ min in a $37^{\circ} \mathrm{C}$ water bath. The cell suspension was transferred to a $50 \mathrm{~mL}$ tube and the vial was rinsed 3 times with Complete iCell Neuronal Maintenance Medium. Total volume 
in the $50 \mathrm{~mL}$ tube was brought to $10 \mathrm{~mL}$ and the tube was turned upside down twice. A sample for cell counting was taken and cells were centrifuged for $5 \mathrm{~min}$ at $1300 \mathrm{rpm}$. The pellet of $\mathrm{iCell}^{\circ}$ neurons was dissolved in dotting medium (i.e. supplemented BrainPhys ${ }^{\mathrm{TM}}$ medium with $10 \%$ laminin) to a solution of 14.000 cells $/ \mu \mathrm{L}$. In parallel, $\mathrm{iCell}^{\circ}$ Glutaneurons were dissolved in dotting medium to a solution of 13.000 cells $/ \mu \mathrm{L}$.

When all cells were thawed or detached, we created two types of co-culture models that differ in the ratio of inhibitory neurons by addition or absence of iCell $^{\circ}$ neurons to create different profiles of neuronal activity (see Tukker et al., 2018 for details). First, a mixture was made of $\sim 13 \%$ iCell $^{\circ}$ astrocytes, $\sim 17 \%$ iCell $^{\circ}$ neurons and $\sim 70 \%$ iCell $^{\circ}$ Glutaneurons (culture model A) according to Table 1 . Cells were plated in $10 \mu \mathrm{L}$ droplets (Table 2). In order to get the total volume to $10 \mu \mathrm{L}, 4.36 \mu \mathrm{L}$ dotting medium was added. For culture model B, a mixture was made of $\sim 15 \% \mathrm{iCell}^{\circ}$ astrocytes and $\sim 85 \% \%$ iCell $^{\circ}$ Glutaneurons (according to Table 1). In this case, 4.65 $\mu \mathrm{L}$ dotting medium was added to bring the total plating volume to $10 \mu \mathrm{L}$. Following plating, droplets were allowed to adhere for $1 \mathrm{hr}$ after which medium was added (Table 2). On DIV1, 50\% of the medium was refreshed with room temperature (RT) supplemented BrainPhys ${ }^{\mathrm{TM}}$ medium. Hereafter, $50 \%$ medium changes took place 3 times a week up till DIV23.

Table 1. Composition of cell models and plating density.

\begin{tabular}{lllll} 
Culture & Cell type and $\%$ & N/well & Solution & $\mu \mathrm{L} /$ well \\
\hline A & $\sim 13 \%$ astrocytes & 10,000 & 14,000 cells $/ \mu \mathrm{L}$ & $0.71 \mu \mathrm{L}$ \\
& $\sim 17 \%$ iCell $^{\circ}$ neurons & 13,000 & 14,000 cells $/ \mu \mathrm{L}$ & $0.93 \mu \mathrm{L}$ \\
& $\sim 70 \%$ iCell $^{\circ}$ Glutaneurons & 52,000 & 13,000 cells $/ \mu \mathrm{L}$ & $4 \mu \mathrm{L}$ \\
B & $\sim 15 \%$ astrocytes & 11,250 & 14,000 cells $/ \mu \mathrm{L}$ & $0.80 \mu \mathrm{L}$ \\
& $\sim 85 \%$ iCell $^{\circ}$ Glutaneurons & 63,750 & 13,000 cells $/ \mu \mathrm{L}$ & $4.55 \mu \mathrm{L}$ \\
\hline
\end{tabular}

Table 2. Cell culture surfaces and medium volume.

\begin{tabular}{lll} 
Culture surface & Experiment & Total volume \\
\hline $\begin{array}{l}\text { 48-well MEA plate (Axion Biosystems Inc, Atlanta, GA, USA) } \\
\mu \text {-slide 8-well chambered coverslip (Ibidi BmbH, Planegg, }\end{array}$ & MEA recording & $300 \mu \mathrm{L} /$ well \\
$\begin{array}{l}\text { Germany) } \\
\text { Glass-bottom dishes (MatTek, Ashland MA, USA) }\end{array}$ & $\begin{array}{l}\text { Live fluorescence } \\
\text { imaging }\end{array}$ & $200 \mu \mathrm{L} /$ well \\
& & \\
\hline
\end{tabular}


Since both co-culture models require several days to develop functional neuronal networks, MEA and imaging experiments should not be performed before DIV11. In our experience, the optimum window for performing MEA and live imaging experiments ranges from DIV14-23. It should be noted that the optimum window for measurements differs between the various available commercial models as well as culture conditions (e.g., cell density and \% astrocytes).

It should be noted that using a high ratio of astrocytes may cause the cells to cluster, complicating imaging and MEA experiments. Developmental curves can be measured from DIV4 onwards and can be used to determine the optimum window for assessing acute neurotoxicity. We strongly recommend to always measure a developmental curve before performing neurotoxicity assessment when starting to work with new cells and/or new culture protocols.

\subsection{Confocal microscopy for immunocytochemistry}

Immunofluorescent images of chemically fixed samples were captured with a Leica DMI4000 TCS SPEII confocal microscope. To capture images, a 20x oil immersion objective (ACS APO IMM NA 0.6) was used. The 20x objective allows for visualisation of multiple neurons in one frame with the connecting dendrites and axons being clearly visible. When a 10x objective is used, too many details and sensitivity are lost, whereas a $40 x$ objective does not capture the complexity of the network structure. It is recommended to visually scan the complete chamber to make sure the selected area is representative for the culture. We noticed that the neuronal co-cultures tend to grow differently at the periphery of the culture area compared to the centre. In order to capture an area that best matches the region where MEA measurements take place, a region in the middle of the dish is chosen. Once a representative area is found, its recommended to define an upper and lower limit in order to make a picture based on z-stacks. We recommend imaging a $\mathrm{z}$-stack series, since the elaborate extensions of the cells are poorly captured in a single plane. An axially extended view provided by a maximal intensity projection visualises the structures better. Images were captured as .lif files using Leica Application Suite Advanced Fluorescence software (LAS AF version 2.6.0; Leica Microsystems GmbH, Wetzlar, Germany).

\subsection{Live cell [Ca2+]i changes using a temperature controlled microscopy unit} Live changes in $[\mathrm{Ca2}+] \mathrm{i}$ were monitored with the fluorescent dye Fura-2AM (Ex: 340 and 380/Em: 510, Life Technologies, Bleiswijk, The Netherlands) using an Axiovert $35 \mathrm{M}$ inverted microscope (Zeiss, Göttingen, Germany) as described 
previously (Heusinkveld et al., 2010). We used a $40 x$ oil immersion objective (PlanNeoFluar NA 1.30) to capture images. Light with an excitation wavelength of 340 and $380 \mathrm{~nm}$ evoked by a monochromator (TILL Photonics Polychrome IV; TILL Photonics GmBH, Gräfelfing, Germany) was directed to the sample via a $290 \mathrm{~nm}$ long-pass filter and beamsplitter. From there, emitted light with a wavelength of $510 \mathrm{~nm}$ was directed to a $440 \mathrm{~nm}$ long-pass filter and was collected every $0.5 \mathrm{~s}$, i.e. at a sample frequency of $2 \mathrm{~Hz}$ for each excitation wavelength, with an Image SensiCam digital camera (TILL photonics $\mathrm{GmBH}$ ). The degree of fluorescence in Fura-2-loaded neuronal co-cultures allowed us to sample with binning $2 \times 2$. TILLvisION (version 4.01) software was used to trigger the light source, camera and data acquisition.

For the cells to exhibit spontaneous network activity, experiments must take place at $37^{\circ} \mathrm{C}$. Below this temperature no spontaneous calcium oscillations are visible (data not shown). In order to keep our samples at a stable temperature, we equipped the microscope with a custom build heating system and bipolar temperature control unit (TC-202, Medical Systems Corp, Greenvale, New York, USA).

Changes in the ratio $\mathrm{F} 340 / \mathrm{F} 380$ of selected regions of interest reflecting the changes in [Ca2+]i were further analysed using custom-made MS Excel macros. Since the cells form networks, spontaneous synchronous calcium oscillations are seen in all regions.

\subsection{Simultaneous live cell imaging of calcium transients and membrane depolarisation}

To monitor calcium levels X-Rhod-1 (Ex: 580/Em: 602, AM-ester derivative, Life Technologies, Bleiswijk, The Netherlands) was used and for membrane depolarisation the dye FluoVolt (Ex: 488/Em: 515, Life Technologies, Bleiswijk, The Netherlands).

Live cell microscopic fluorescence recordings were performed on a commercial NIKON Ti system equipped with an EMCCD camera (iXon Ultra897, ANDOR) using wide field illumination. A 10x CFI Plan Fluor air objective (NA 0.3, WD $16 \mathrm{~mm}$ ) was applied to record the data. A LED light source (Lumencor Spectra X) illuminated the samples to deliver $470 / 24 \mathrm{~nm}$ and $575 / 25 \mathrm{~nm}$ light bandwidths sequentially. Emitted fluorescence light was collected through a quadband emission filter (Chroma, DAPI/FITC/TRITC/Cy5 Quad). Triggered synchronization of the LEDs and the camera was directed with the NIS elements 
software ND acquisition module (NIKON, version 4.6) via a NIDAQ communication board (National Instruments). Halogen illumination light path was regulated by a fast shutter (Lambda SC, SUTTER Instruments) to obtain differential interference contrast (DIC) images without the delay of slow on and off glowing rates of these lamps. Fast focus control is performed by a piezo stepping stage device (Mad City labs, Madison, WI, USA).

In the Spectra-X module of the NIS element software, the $470 \mathrm{~nm}$ and $575 \mathrm{~nm}$ light sources were controlled. Via the triggered acquisition module light levels and exposure times were set $(<3 \%, \mathrm{ND} 4$ and 8 filters were available for careful illumination modulation). The EMCCD camera capture area was cropped to $256 \times 256$ and $2 \times 2$ binning was applied at $20 \mathrm{~ms}$ exposures (sampling in frame transfer mode at $17 \mathrm{Mhz}$ horizontal pixel shift read out frequency with $300 \mathrm{~V}$ amplification gain.)

The system is equipped with temperature and $\mathrm{CO}_{2}$-control in a stage top setup with a small water container to provide a humidified culture environment (TOKAI HIT, INUBG2EH-TiZB). The lens heating option was omitted for the air objectives employed.

Both the automated focus control system of NIKON (perfect focus system or pfs) and the compressor that provides air for the vibration isolation table can create vibrations and were thus switched off for the recordings.

Following data acquisition, recorded data was exported to MS Excel. For further frequency data analysis one representative trace from a dish was chosen as all cells display synchronized oscillations in calcium levels and membrane potential. To calculate changes in peak amplitude, data was loaded in a custom-made MatLab program and averages of multiple responsive cells in the dish were calculated. Data are reported as mean \pm SEM form $n$ cells.

\subsection{Multi-well micro-electrode array recordings}

Cells were cultured on 48-well MEA plates (Fig. 1) as described previously (Nicolas et al., 2014; Hondebrink et al., 2016). Each well contains an electrode array of 16 nanotextured gold micro-electrodes that are $\sim 40-50 \mu \mathrm{m}$ in diameter with a $350 \mu \mathrm{m}$ centre-to-centre spacing. Each well contains 4 integrated ground electrodes. In total this yields 768 channels in one plate that can be recorded simultaneously. A Maestro 768-channel amplifier with an integrated heating system, temperature controller and data acquisition interface (Axion Biosystems Inc., Atlanta, GA, USA) was used 
for recordings of neuronal activity. Data acquisition was managed with Axion's Integrated Studio (AxIS version 2.4.2.13) and recorded as .RAW files. Files were obtained by sampling all channels simultaneously with a gain of $1200 \mathrm{x}$ and a sampling frequency of $12.5 \mathrm{kHz} /$ channel using a band-pass filter $(200-5000 \mathrm{~Hz})$. Notably, the large number of electrodes, sampled at high frequency, will yield a large amount of data ( $1 \mathrm{~GB} / \mathrm{min}$ recording). Consequently, sufficient storage space should be available.

For data analysis . RAW files were re-recorded to obtain Alpha Map files. During this re-recording, spikes were detected using the AxIS spike detector (Adaptive threshold crossing, Ada BandFIt v2). A variable threshold spike detector was used with a threshold set at $7 \mathrm{x}$ standard deviation of the internal noise level (rms) on each electrode. The obtained spike files were loaded in Neural Metric (version 2.04, Axion Biosystems). In this program, bursts were detected with the Poisson Surprise method (minimal surprise S=10 (Legéndy and Salcman, 1985)). Network bursts were extracted with the adaptive threshold method (min \# of spikes 10; min \% electrodes 25 ).

The Neural Metric output files (.csv files) were loaded in a custom-made MS Excel macro. We only used active electrodes (MSR $\geq 6$ spikes/min) from active wells ( $\geq 1$ active electrode) for further analysis. Electrodes were seen as bursting electrodes when the minimum burst rate was $\geq 0.001$ bursts/sec. Only wells with $\geq 2$ bursting electrodes were included for network bursting and synchronicity analysis. Effects of test compounds were determined by comparing the activity of the baseline of a well to the activity in that well following exposure. During data analysis it is important to correct for exposure artefacts (Hondebrink et al., 2016). To do so, the time it took to expose all wells was excluded from data analysis. For example, if exposing the plate took $2 \mathrm{~min}$, the first two min of the recording were not used for data analysis, but only the subsequent $30 \mathrm{~min}$. Then, treatment ratios per well for different metric parameters (Mean spike rate [MSR]; mean burst rate $[M B R]$ and mean network burst rate [MNBR]) were calculated by expressing the parameterexposure as a percentage change of parameterbaseline. Next, treatment ratios were normalized to vehicle control. Outliers were defined as not within average $\pm 2 \mathrm{x}$ standard deviation and excluded for further analysis. MEA data are expressed as mean \pm SEM. A one-way ANOVA was performed to determine statistical significant changes $(\mathrm{p}<0.05)$ in MSR, MBR and MNBR compared to vehicle control. 

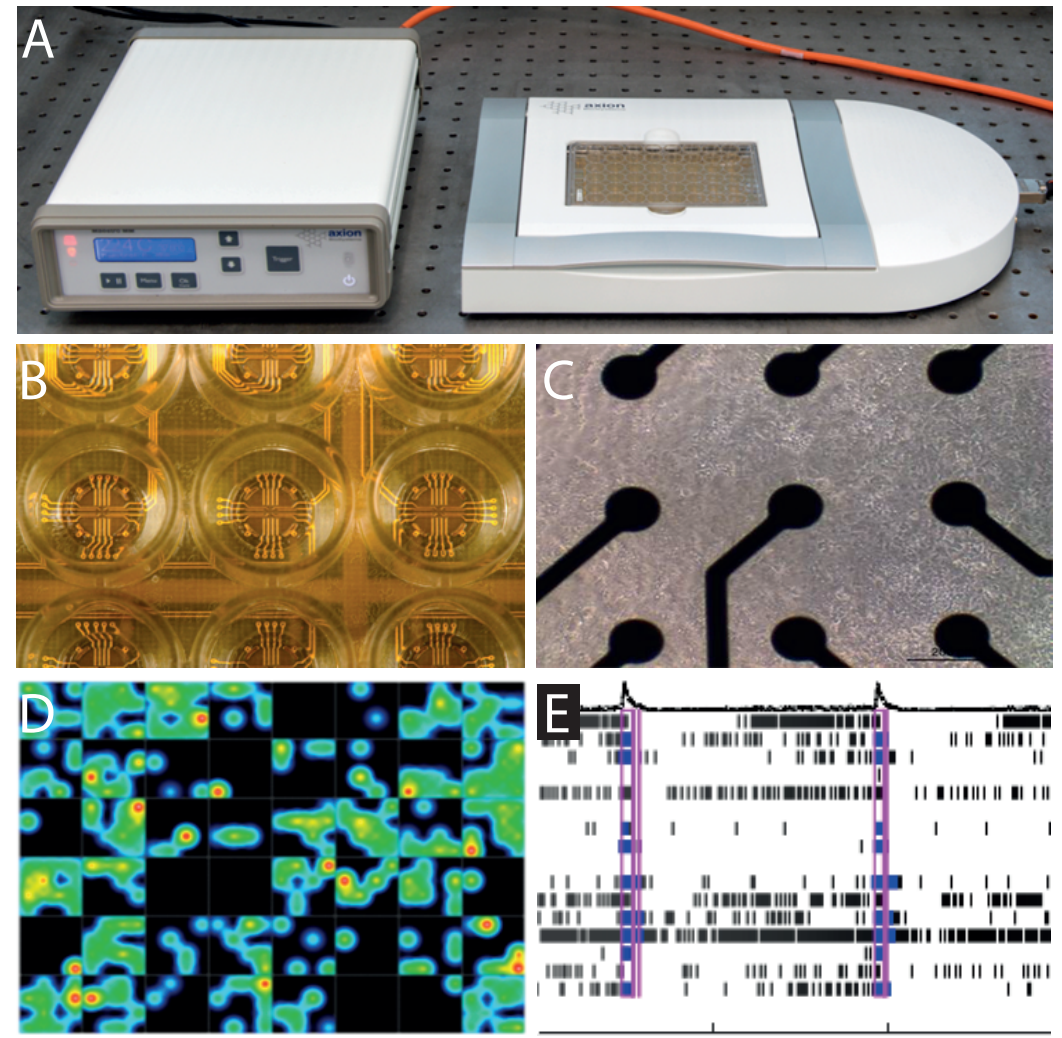

Figure 1. Experimental setup for measurements of spontaneous neuronal network activity. Axion's Maestro platform (A) was used to record neuronal activity of co-cultures grown in 48-well MEA plates (B). Each well contains an electrode grid with 16 electrodes/well (C) for non-invasive extracellular field recordings. Live heat maps are shown in AxIs software during recordings (D). Files are loaded in neural metric to determine (E) spikes (black), bursts (blue) and network bursts (pink squares). 


\section{METHODS AND RESULTS}

\subsection{Immunocytochemistry: visualising neuronal networks}

Neuronal networks of culture model A were chemically fixed and specific antibodies were used to demonstrate the presence of neurons and astrocytes. Neurons were identified using antibodies against class III $\beta$-tubulin, which is found almost exclusively in neurons. Anti-s1oo $\beta$ is a protein specific for glial cells and was used to identify astrocytes. Notably, many other antibodies can be used to gain additional insight in the composition of the neuronal network. For example, using antibodies against vGluT (vesicular glutamate transporter), vGAT (vesicular GABA transporter) or tyrosine hydroxylase (marker for dopaminergic neurons) will provide information on the types of neurons present.

In order to stain the cultures, a staining protocol described previously (Tukker et al., 2018) was used. The cultures were chemically fixed at DIV16 and 21 with $4 \%$ PFA in 0.1 M PBS (pH 7.4) for 15 min at RT. We found that longer fixation times reduced epitope recognition by the antibodies. Following fixation, chambered coverslips were quenched for PFA, permeabilized and incubated for $20 \mathrm{~min}$ at RT with $20 \mathrm{mM} \mathrm{NH} 4 \mathrm{Cl}$ in blocking buffer ( $2 \%$ bovine serum albumin and $0.1 \%$ saponin in PBS). Hereafter, chambers were incubated overnight at $4^{\circ} \mathrm{C}$ with the primary antibody. The following primary antibodies were used: mouse anti-S10o $\beta$ (final dilution1:500; Ab11178, Abcam, Cambridge, United Kingdom) to stain astrocytes and rabbit anti- $\beta$ III tubulin (final dilution 1:250; Ab18207, Abcam, Cambridge United Kingdom) to visualise the iCell ${ }^{\circledast}$ neurons and iCell ${ }^{\circledR}$ Glutaneurons. The following day, chambers were washed thrice with blocking buffer and incubated with donkey anti-rabbit Alexa Fluor ${ }^{\circledR} 488$ (final dilution 1:100; 715-545-152, Life Technologies, Bleiswijk, The Netherlands) and donkey anti-mouse Alexa Fluor ${ }^{\circledR} 594$ (final dilution 1:100; 715-585-151, Life Technologies, Bleiswijk, The Netherlands) for $45 \mathrm{~min}$ at RT in the dark. During the last 2-3 min of the incubation time $200 \mathrm{nM}$ DAPI (staining the nuclei) was added. Chambers were washed again $3 x$ with blocking buffer and sealed with 2-3 droplets of FluorSave (Calbiochem, San Diego, CA, USA). Now the chambers are ready for use. However, they can be stored for months at $4^{\circ} \mathrm{C}$ in the dark until use.

The images of the chemically fixed and stained co-cultures of culture model A show that mixed neuronal networks with a high degree of complexity are formed (Fig. $2 \mathrm{AB}$ ). It is clear that the network is already strongly developed at DIV16 (Fig. 2A) and this is maintained till DIV21 (Fig. 2B). The images also show that astrocytes 
do not overgrow the neuronal population. The ratio astrocytes to neurons remains comparable over time and is in line with the plated ratio. Altogether, these results indicate that this co-culture consists of a mixed cell population with neurons as well as supporting astrocytes that can be used for neurotoxicity testing.

\subsection{Live cell imaging: $\left[\mathrm{Ca}^{2+}\right]_{i}$ changes in hiPSC-derived co-cultures}

Both dyes used for the $\left[\mathrm{Ca}^{2+}\right]_{i}$ experiments described in this chapter contain an acetoxymethyl (AM) ester. The AM group allows the dye to cross the cell membrane, which allows the cells to be loaded in a non-invasive manner. Following membrane crossing, the AM group is cleaved from the dye by non-specific esterases in the cytosol. The cleaved dye is no longer able to cross the cell membrane and remains in the cytosol. The calcium-sensitive dye Fura-2 is fluorescent green (510 nm) and fluorescence increases upon $\mathrm{Ca}^{2+}$ binding following excitation at $340 \mathrm{~nm}$, but decreases fluorescence by excitation at $380 \mathrm{~nm}$. The resulting F340/380 ratio thus correlates directly with the $\left[\mathrm{Ca}^{2+}\right]_{\mathrm{i}}$.

Cells cultured in glass-bottom dishes of culture model B were loaded with 5 $\mu \mathrm{M}$ Fura-2AM for $1 \mathrm{hr}$ at $37^{\circ} \mathrm{C}$ in a humidified $5 \% \mathrm{CO}^{2}$ incubator. After loading, cells were washed 4 times with $37^{\circ} \mathrm{C}$ saline solution to remove excess dye. Then dishes with loaded cells were placed on the stage of the inverted microscope in the heating ring. The temperature sensor was placed in the dish and cells were allowed to warm till $37^{\circ} \mathrm{C}$. As soon as this temperature was reached, a $5 \mathrm{~min}$ recording was started to measure spontaneous calcium oscillations.

With this method it is possible to detect spontaneous calcium oscillations in hiPSC-derived neuronal co-cultures (Fig. 3) Sample traces indicate the presence of spontaneous calcium oscillations. Cells oscillate synchronously, indicating that cells are all part of a single network.

\subsection{Live cell imaging: simultaneous calcium oscillations and membrane depolarisation in hiPSC-derived co-cultures}

X-rhodamine-1 (X-Rhod-1) was used to study spontaneous calcium oscillations. This dye works in a comparable manner as Fura-2AM. However, the main difference is that Fura-2AM is a dual wavelength dye and X-Rhod-1 a single wavelength dye in the red colour range (emission $595 \mathrm{~nm}$ ). The latter has as advantage that it can be imaged at a higher sample frequency and allowing it to be used in combination with other sensor dyes. In order to study simultaneous changes in membrane depolarisation, we used the dye FluoVolt. 


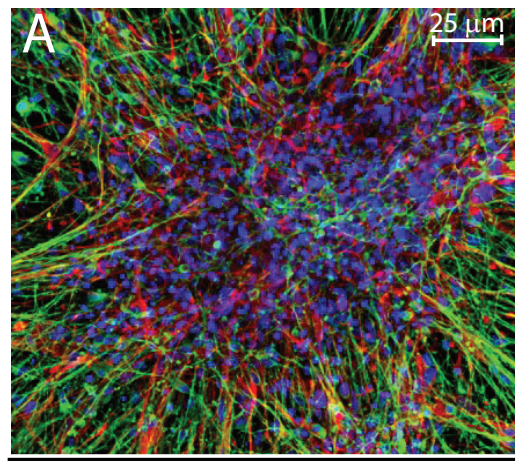

DIV $16-\beta(I I I) T u b u l i n$ / S100 $\beta$ / DAPI

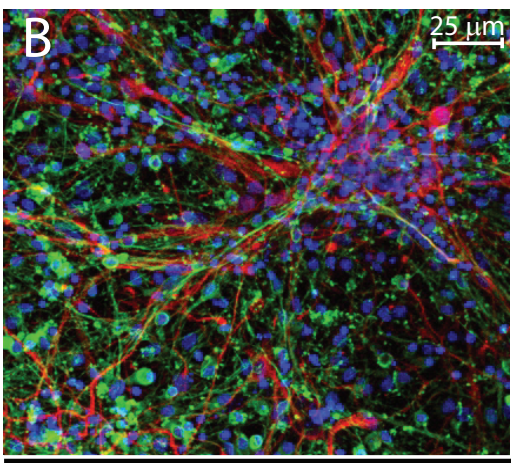

DIV 21 - $\beta$ (III)Tubulin / S100 $\beta$ / DAPI

Figure 2. Immunofluorescent stainings of co-culture model A. At DIV16 (A) and DIV21 (B) cultures were stained with $\beta($ III)tubulin (green) and $s 100 \beta$ (red) to identify respectively neurons and astrocytes in the co-culture. Nuclei were stained with DAPI (blue). Scale bar depicts $25 \mu \mathrm{m}$.

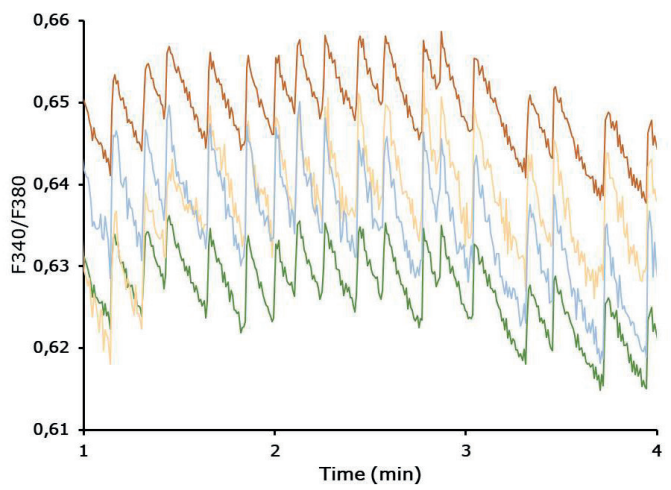

Figure 3. Sample traces of representative neuronal cells (DIV23) showing spontaneous calcium oscillations. Each trace represents the oscillations of a single cell over a 3 min time period. Cells oscillate synchronously, indicating they are part of a single network. 
In general, there are two types of probes that can be used to do this: fast or slow response probes. The first type reacts fast, but the magnitude of potentialdependent fluorescence change is small. The latter type reacts slower, but the magnitude of fluorescence fluctuation is high. FluoVolt is a dye that combines characteristics of fast and slow probes as it reacts fast (millisecond time scale) and yields a high magnitude of fluorescence with a small change in membrane potential $(\sim 25 \%$ change in fluorescence per $100 \mathrm{mV}$ change in membrane potential).

Cells of culture model B were cultured up till DIV21 in glass-bottom dishes and used to record changes in calcium transients and membrane voltage fluctuations. One day prior to measurements, cells were transferred to an incubator close to the imaging station to minimize transport stress and temperature variation at the time of the experiment. At the day of measurements, start the microscope peripheral modules (SpectraX, Mad City Lab Stage control, XY control joystick unit, halogen lamp and TOKAI HIT control unit) before powering the microscope stand and finally turn on the PC. Distilled water was placed in the basin of the TOKAI HIT sample holder. It is important to place a dummy sample in the holder to prevent condensation on optics below the table. At this point, the climate control unit ( $370 \mathrm{C}$ for the basin, $400 \mathrm{C}$ for the TOP deck heating, $5 \% \mathrm{CO} 2$ ) is switched on and the whole setup is left for acclimatization for $30 \mathrm{~min}$. All experiments took place at $37^{\circ} \mathrm{C}$.

During the acclimatization time, cultures were loaded with a mixture of $5 \mu \mathrm{M}$ $\mathrm{X}$-Rhod-1, $5 \mu \mathrm{M}$ FluoVolt and PowerLoad solution (a solubilizing agent provided in FluoVolt kit) in life cell imaging solution (LCIS; $140 \mathrm{mM} \mathrm{NaCl}, 2.5 \mathrm{mM} \mathrm{KCl}, 1.8 \mathrm{mM}$ $\mathrm{CaCl} 2,1.0 \mathrm{mM} \mathrm{MgCl}$, $20 \mathrm{mM}$ HEPES, $20 \mathrm{mM}$ glucose) for 15-20 min. Following incubation, cells were washed 4 times with $1 \mathrm{~mL}$ LCIS. After loading, the sample was placed in the TOKAI HIT holder (UNIVD35) to search for a representative area and select regions of interest (ROIs) to obtain live intensity preview plots (Fig. 4). Next, a 10 min baseline recording was made prior to addition of test compound and a subsequent $10 \mathrm{~min}$ exposure recording. For exposure recordings, a fresh stock solution of picrotoxin (PTX) in EtOH was prepared on the day of the experiment. Stock solution was further diluted in LCIS. Solvent concentration did not exceed $0.1 \% \mathrm{v} / \mathrm{v}$. 
Data again confirm that neuronal co-cultures form networks, since oscillations in membrane potential as well as calcium occur for all cells at the same time (Fig. 5A/B left). Exposure (dilution 1:10) to $10 \mu \mathrm{M}$ (PTX) does not affect the frequency of oscillations. However, it does result in a decrease of the amplitude of membrane potential peaks to $57.2 \%( \pm 3.4, \mathrm{n}=6$ ) of baseline (Fig. $5 \mathrm{~A})$. A stronger effect is seen on calcium oscillations, where amplitude decreases to $37.5 \%( \pm 3.2, n=6)$ compared to baseline (Fig. 5B). Basal fluorescence increases over time, likely as a result of ongoing de-esterification of dye that still contained the AM module. However, filter and/or subtraction methods can be used to eliminate this trend in fluorescence from data analysis.
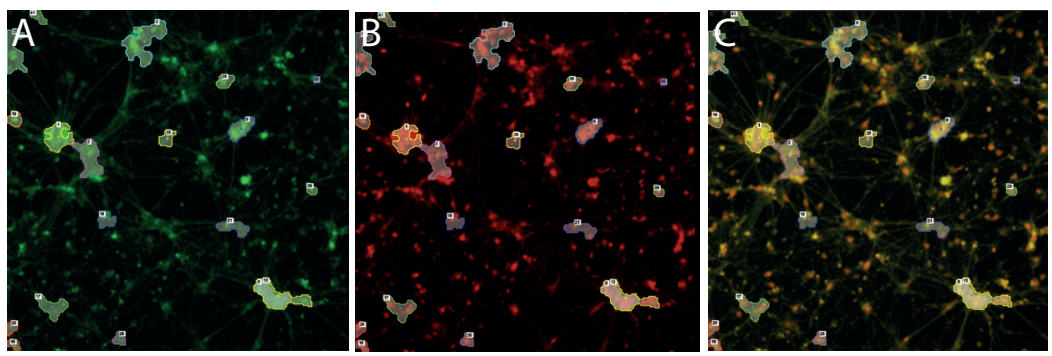

Figure 4. Captures of neuronal cells loaded with FluoVolt (A), X-Rhod-1 (B) and an overlay (C). Encircled areas are selected regions of interest for measurements as further illustrated in figure 5.

Table 3. Development of spontaneous neuronal activity and (network) bursting at different DIVs. Data are expressed as mean \pm SEM.

\begin{tabular}{lllllll} 
& \multicolumn{2}{c}{ MSR } & \multicolumn{2}{c}{ MBR } & \multicolumn{2}{c}{ MNBR } \\
& $\begin{array}{l}\text { Frequency } \\
\text { (Hz) }\end{array}$ & $\begin{array}{l}\text { \% active } \\
\text { wells }\end{array}$ & $\begin{array}{l}\text { Frequency } \\
(\mathbf{H z})\end{array}$ & $\begin{array}{l}\text { \% active } \\
\text { wells }\end{array}$ & $\begin{array}{l}\text { Frequency } \\
(\mathrm{Hz})\end{array}$ & $\begin{array}{l}\text { \% active } \\
\text { wells }\end{array}$ \\
\hline DIV7 & $1.53 \pm 0.14$ & 93.8 & $0.04 \pm 0.01$ & 26.7 & $0.01 \pm 0.01$ & 25 \\
DIV14 & $1.43 \pm 0.14$ & 93.8 & $0.02 \pm 0$ & 13.3 & $0.01 \pm 0.01$ & 100 \\
& & & & & & \\
DIV21 & $1.29 \pm 0.18$ & 93.8 & $0.05 \pm 0.01$ & 76.7 & $0.03 \pm 0.03$ & 65.2 \\
\hline
\end{tabular}



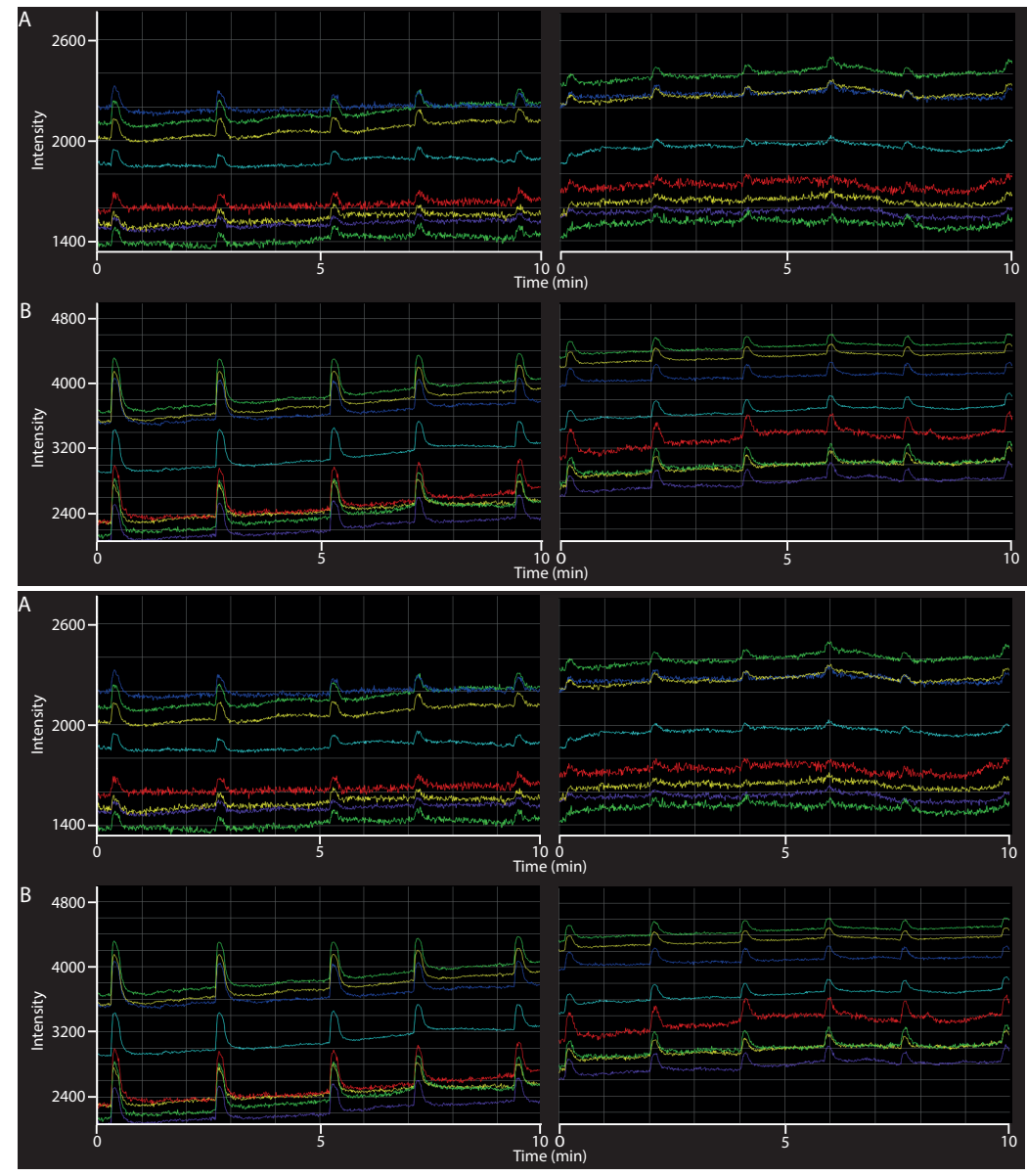

Figure 5. Selection of traces of cells selected in figure 4 for assessing changes in fluorescence of FluoVolt (A) and X-Rhod-1 (B) during baseline (top) and exposure (bottom) to $10 \mu \mathrm{M}$ PTX. Different cells oscillate simultaneously, indicating that changes in membrane potential (A) and $\left[\mathrm{Ca}^{2+}\right]_{i}(\mathrm{~B})$ occur at the same time and the cells are part of the same neuronal network. 


\subsection{MEA: assessing spontaneous neuronal network activity in hiPSC-derived co-cultures}

All MEA measurements took place at $37^{\circ} \mathrm{C}$ with culture model B. Experiments took place at DIV21 since this is the optimum window in terms of activity (Table 3). It should be noted that different culture models develop differently and may therefore have a different optimum window. For this reason, it is strongly recommended to always make a developmental curve before starting toxicity experiments. In order to determine effects of test compounds on spontaneous network activity and (network) bursting of the hiPSC-derived co-culture, a $30 \mathrm{~min}$ baseline recording was made. Prior to the $30 \mathrm{~min}$ recording, plates were allowed to equilibrate in the Maestro for $\sim 5 \mathrm{~min}$. Immediately following this $30 \mathrm{~min}$ baseline recording, cells were exposed (dilution 1:10) to the test compounds or the solvent control and another $30 \mathrm{~min}$ recording was made. Each well was exposed only once, since cumulative dosing may confound results due to e.g. receptor (de)sensitization. We prepared fresh PTX stock solutions in $\mathrm{EtOH}$ and of strychnine in supplemented BrainPhys $\mathrm{s}^{\mathrm{Tm}}$ medium prior to every experiment. Stock solutions of PTX were further diluted in supplemented BrainPhys ${ }^{\mathrm{TM}}$ medium such that solvent concentration never exceeded $0.1 \% \mathrm{v} / \mathrm{v}$.

Neuronal activity in culture model B can be modulated with strychnine (Fig. 6A). Exposure to the highest tested concentration strongly decreases MSR, MBR and MNBR (Fig. 6A, right) as compared to baseline activity (Fig. 6A, left). The inhibitory effect of strychnine on MSR is concentration-dependent (Fig. 6B). This in contrast to the MBR, which increases following exposure to low concentrations of strychnine and decreases following exposure to higher concentrations. MNBR decreases by all tested concentrations of strychnine. Exposure to PTX (Fig. 6B) has little effect on the MSR, except for exposure to $1 \mu \mathrm{M}$, which decreases the MSR. The lowest test concentration PTX causes a decrease in MBR, however with increasing concentrations, the MBR increases as well. On the other hand, all tested concentrations of PTX cause a decrease in MNBR.

The different effects that strychnine and PTX have on culture model B become clear from the inclusion of additional metric parameters illustrated by a heat map (Fig. 7). This heatmap also indicates that for proper MEA data analysis it is important to include more parameters than just MSR, MBR and MNBR. 

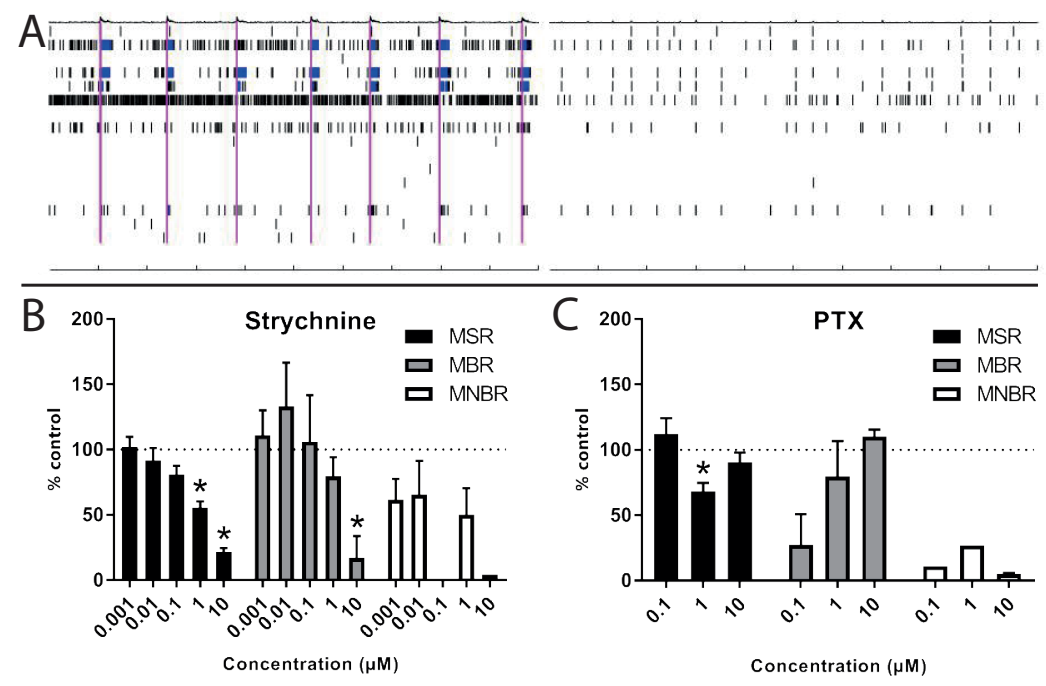

Figure 6. Toxicological modulation of spontaneous network activity, bursting and network bursting. Cultures were exposed at DIV21 to strychnine (A/B) or PTX (C). Spike raster plot depicting activity and (network) bursting before (left) and following exposure (right) to $10 \mu \mathrm{M}$ strychnine (A). Each row depicts one electrode, each tick representing one spike (field potential) in a $200 \mathrm{~s}$ interval, bursts are depicted in blue and network bursts encircled in pink squares. Data are expressed as MSR, MBR or MNBR as \% change relative to vehicle control; mean \pm SEM from $\mathrm{n}=1-12 ; " p<0.05(\mathrm{~B} / \mathrm{C})$.

\section{CONCLUSIONS}

Human iPSC-derived co-cultures develop functional and spontaneously active neuronal networks consisting of mature neurons (Hyysalo et al., 2017; Paavilainen et al., 2018). Our immunocytochemistry data demonstrate the mixed nature of our co-culture models consisting of neurons and astrocytes that form complex, multicellular networks (Fig. 2).

We have shown that hiPSC-derived neuronal co-cultures are amenable to multiple real-time recording techniques, including live cell imaging and electrophysiology. Our calcium imaging data indicate that the co-culture models develop spontaneous calcium oscillations and spontaneous changes in membrane potential (Fig. 3/5). Since calcium oscillations and changes in membrane potential occur in multiple cells at the same time (Fig. 3/5), it can be concluded that functional networks are 


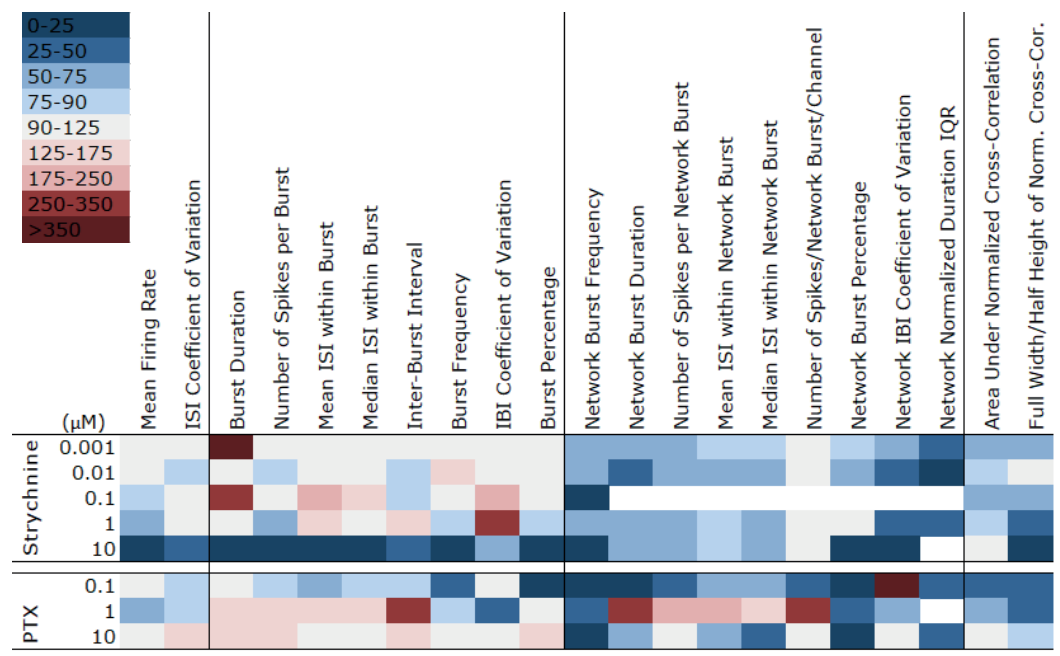

Figure 7. Heatmap of the effects of strychnine and PTX (concentration in $\mu \mathrm{M}$ ) on selected metric parameters on culture model B. Colour scaling is based on the magnitude of the $\%$ of change relative to the vehicle control. No average could be calculated for white cells.

formed. Moreover, MEA recordings demonstrate that neuronal co-cultures develop spontaneous network activity and (network) bursting (Table 3/ Fig. 6).

Spontaneously active human iPSC-derived neuronal co-cultures are suitable for (preliminary) neurotoxicity assessment (Tukker et al., 2016, 2018; Hondebrink et al., 2017; Ishii et al., 2017; Kasteel and Westerink, 2017) as is confirmed by our data (Fig. 5-7). However, it must be noted that model composition, e.g. the ratio of GABAergic and glutamatergic neurons and the presence of astrocytes, greatly influences the model's characteristics (Tukker et al., 2018). Therefore, a careful model characterization must be performed prior to toxicity testing.

The increasing availability of hiPSC from different donors and/or patients differentiated in different cell types, e.g. GABAergic, glutamatergic, dopaminergic neurons and astrocytes as well as peripheral neurons, holds great promise for future personalized toxicity and safety screening. Using hiPSC-derived neurons in combination with the techniques described here will provide a good starting point for neurotoxicity assessment. 


\section{ACKNOWLEDGEMENTS}

We gratefully acknowledge members of the Neurotoxicology Research Group for helpful discussions. This work was funded by a grant from the National Centre for the Replacement, Refinement and Reduction of Animals in Research (NC3Rs; project number 50308-372160), the Netherlands Organisation for Health Research and Development (ZonMW; InnoSysTox project number 114027001) and by the Faculty of Veterinary Medicine (Utrecht University, The Netherlands). 
S 
Part II

\section{SINGLE RECEPTOR}

TO FULL NETWORK 
Chapter 4

Perfluorooctane sulfonate (PFOS) and perfluorooctanoate (PFOA) acutely

affect human $\alpha_{1} \beta_{2} \gamma_{2 \mathrm{~L}} \mathrm{GABA}_{\mathrm{A}}$ receptor and spontaneous neuronal network function in vitro

Anke M. Tukker, Lianne M.S. Bouwman, Regina G.D.M. van Kleef, Hester S. Hendriks, Juliette Legler and Remco H.S. Westerink

Neurotoxicology Research Group, Toxicology Division, Institute for Risk Assessment Sciences (IRAS), Faculty of Veterinary Medicine, Utrecht University, P.O. Box 80.177, NL-3508 TD Utrecht, The Netherlands

Scientific Reports (2020), volume 10

DOI:10.1038/s41598-020-62152-2

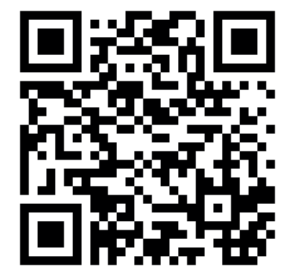




\begin{abstract}
Concerns about the neurotoxic potential of polyfluoroalkyl substances (PFAS) such as perfluorooctane sulfonate (PFOS) and perfluorooctanoic acid (PFOA) increase, although their neurotoxic mechanisms of action remain debated. Considering the importance of the $\mathrm{GABA}_{\mathrm{A}}$ receptor in neuronal function, we investigated acute effects of PFAS on this receptor and on spontaneous neuronal network activity.
\end{abstract}

PFOS (Lowest Observed Effect Concentration (LOEC) $0.1 \mu \mathrm{M}$ ) and PFOA (LOEC $1 \mu \mathrm{M}$ ) inhibited the GABA-evoked current and acted as non-competitive human $\mathrm{GABA}_{\mathrm{A}}$ receptor antagonists. Network activity of rat primary cortical cultures increased following exposure to PFOS (LOEC $100 \mu \mathrm{M}$ ). However, exposure of networks of human induced pluripotent stem cell (hiPSC)-derived neurons decreased neuronal activity.

The higher sensitivity of the $\alpha_{1} \beta_{2} \gamma_{2 \mathrm{~L}} \mathrm{GABA}_{\mathrm{A}}$ receptor for PFAS as compared to neuronal networks suggests that PFAS have additional mechanisms of action, or that compensatory mechanisms are at play. Differences between rodent and hiPSC-derived neuronal networks highlight the importance of proper model composition. LOECs for PFAS on $\mathrm{GABA}_{\mathrm{A}}$ receptor and neuronal activity reported here are within or below the range found in blood levels of occupationally exposed humans. For PFOS, LOECs are even within the range found in human serum and plasma of the general population, suggesting a clear neurotoxic risk.

Keywords: PFAS, GABA $A_{A}$ receptor, patch-clamp, spontaneous network activity, micro-electrode array (MEA), human induced pluripotent stem cell (hiPSC)derived neurons 


\section{INTRODUCTION}

Perfluorooctane sulfonate (PFOS) and perfluorooctanoic acid (PFOA) are wellknown perfluoroalkyl substances (PFAS) consisting of an eight-carbon chain in which hydrogen atoms have been substituted with fluorine. Their combined hydrophobic, hydrophilic, oleophobic and lipophobic properties make them ideal industrial surfactants for the manufacturing of consumer products, including paint, stain repellents, fire-fighting foams, and non-stick cookware coatings. The strong carbon-fluorine bond renders PFOS and PFOA highly persistent and studies have shown their presence in the environment, wildlife and even human blood (for reviews see Lindstrom et al., 2011; Grandjean and Clapp, 2015). By 2002, production of PFOS was phased out and production phase out of PFOA followed in 2006 (Environmental Protection Agency Federal Facilities Restoration and Office, 2017). Recent studies indicate that these efforts may be responsible for a reduction in human blood levels in some areas, but the long half-lives of PFOS and PFOA result in slow elimination from environment and humans (Olsen et al., 2007; Li et al., 2018).

Research has demonstrated that the (developing) nervous system is one of the most sensitive targets for PFOS and PFOA. In mice and rats exposed pre- and/ or neonatally to PFOS or PFOA, increased motor activity, decreased habituation and deficits in spatial learning and memory abilities have been observed (Fuentes et al., 2007; Johansson et al., 2008; Butenhoff et al., 2009; Onishchenko et al., 2011; Wang, Liu, et al., 2015; Goulding et al., 2017). Developmental neurotoxicity has also been observed in other species, including chicken (Pinkas et al., 2010) and zebrafish larvae (Ulhaq et al., 2013; Spulber et al., 2014). However, epidemiological studies have been inconclusive on the risks of PFAS exposure on neurodevelopment. Some studies indicate an association between prenatal PFOS and/or PFOA exposure and an increased risk on congenital cerebral palsy (Liew et al., 2014), neuro-behavioural development (Høyer et al., 2015) and visual motor abilities (Harris et al., 2018), whereas others reported no association between PFOS and PFOA exposure on psychomotor, cognitive and neurobehavioural development (Forns et al., 2015), IQ levels (Liew et al., 2018) or increased risk on ADHD and childhood autism (Liew et al., 2015). Notably, most early studies reported no or limited neurodevelopmental or neurological effects of PFAS exposure (Strøm et al., 2014; Forns et al., 2015; Høyer et al., 2015; Goudarzi et al., 2016; Quaak et al., 2016), although recent epidemiological studies indicate an association between PFAS exposure and child behaviour (Ghassabian et al., 
2018; Lenters et al., 2019). It is important to keep in mind that exposure to PFAS in these studies is continuous and/or based on prolonged exposure paradigms.

The mechanism(s) underlying the observed neurotoxicity have been studied in vitro in neuronal cells. At (high) micromolar concentrations, PFOS and/or PFOA can affect a range of (pre)-synaptic processes, including reduction of cell viability (Zhang et al., 2011; Reistad et al., 2013; Chen et al., 2014; Berntsen et al., 2017), altered neuronal differentiation (Slotkin et al., 2008), increased formation of reactive oxygen species (Lee et al., 2012; Reistad et al., 2013; Chen et al., 2014; Mashayekhi et al., 2015; Wang, Nie, et al., 2015; Sun et al., 2018), and increased intracellular calcium $\left(\mathrm{Ca}^{2+}\right)$ concentrations (Liu et al., 2011; Yang et al., 2015). In addition, PFOS and PFOA may also affect postsynaptic processes, including altered glutamate-activated currents and increase of potassium currents (Liao, Cui, et al., 2009; Liao, Wang, et al., 2009). By inducing influx of $\mathrm{Ca}^{2+}$ through voltage-dependent calcium channels, PFOS may exhibit acute excitotoxic effects on synaptic function and chronically inhibit synaptogenesis in rat brain hippocampal neurons (Liao et al., 2008). Although in vivo studies suggest the involvement of the cholinergic (Johansson et al., 2008) and glutamatergic system (Zhang et al., 2019) in PFOS and PFOA mediated neurotoxicity, additional studies on postsynaptic receptors and channels by PFOS or PFOA are lacking.

Previous studies have shown agonistic effects on the postsynaptic human $\mathrm{GABA}_{\mathrm{A}}$ receptor by several persistent organic pollutants, including polychlorinated biphenyls and brominated flame retardants (Antunes Fernandes, Hendriks, van Kleef, Reniers, et al., 2010; Antunes Fernandes, Hendriks, van Kleef, van den Berg, et al., 2010; Hendriks et al., 2010, 2012). The GABA A receptor is the main inhibitory neurotransmitter receptor in the central nervous system and is critical for brain development (Represa and Ben-Ari, 2005), long-term potentiation and synaptic plasticity (Fujii et al., 2000), but also for functional neuronal signal transduction. Because the $\mathrm{GABA}_{\mathrm{A}}$ receptor is the most important inhibitory receptor in the central nervous system, any effect of PFAS on this receptor may in turn affect neuronal signal transduction and spontaneous neuronal network activity.

In the present study we therefore used Xenopus oocytes that express the human $\alpha_{1} \beta_{2} \gamma_{2 L}$ GABA $_{A}$ receptor, which is the most abundant subunit combination (Olsen and Sieghart, 2009), to investigate the acute effects of PFOS and PFOA using the two-electrode voltage-clamp technique. Additionally, we investigated whether the effects of PFOS and PFOA on the $\alpha_{1} \beta_{2} \gamma_{2 L}$ GABA $_{A}$ receptor are reflected in 
the level of spontaneous neuronal network activity using micro-electrode array (MEA) recordings in rat primary cortical neuronal networks, the current gold standard for MEA assays. These experiments were followed by measurements in human iPSC-derived neuronal models to investigate whether they were similarly affected by PFAS exposure as the rodent model.

\section{RESULTS}

\subsection{Antagonistic effects of PFAS on human GABA $A_{A}$ receptor}

Oocytes expressing the human $\alpha_{1}, \beta_{2}, \gamma_{2 L}$ subunits were superfused with various concentrations of GABA to derive a concentration-response curve with an $\mathrm{EC}_{20}$, $\mathrm{EC}_{50}$ and Hill slope of $36 \mu \mathrm{M}, 103 \mu \mathrm{M}$ and 1.3, respectively (see Fig. S1 and in line with previous publications (Hondebrink et al., 2015)). The expression and functionality of the $\gamma_{2 \mathrm{~L}}$ subunit was demonstrated by co-exposing GABA-responsive oocytes to a GABA-diazepam co-exposure (see Fig. S1).

To investigate whether PFOS and PFOA are able to activate the human $\mathrm{GABA}_{\mathrm{A}}$ receptor, GABA-responsive oocytes were superfused with saline containing $0.1-100 \mu \mathrm{M}$ PFOS or PFOA. Neither PFOS nor PFOA induced any ion current, demonstrating that PFOS and PFOA by themselves are not agonists of the $\mathrm{GABA}_{\mathrm{A}}$ receptor (data not shown). To determine possible partial agonistic or antagonistic effects, PFOS and PFOA were co-applied with a low concentration of GABA ( $\tilde{E}_{20}$ ) and the lowest observed effect concentrations (LOEC; defined as the lowest test concentration that is significantly different from the control) of PFOS and PFOA were calculated. At this low effective GABA concentration, both PFOS (LOEC $0.1 \mu \mathrm{M}, \mathrm{IC}_{50} 0.28 \mu \mathrm{M}, \mathrm{CI} 0.22-0.34 \mu \mathrm{M}$, see Fig. 1a, e and Table 1; $\mathrm{F}(1,21)=10.2, p=0.004)$ and PFOA (LOEC $10 \mu \mathrm{M}, \mathrm{IC}_{50} 22 \mu \mathrm{M}$, CI 19.28-24.21 $\mu \mathrm{M}$, see Fig. Ib, f and Table 1; $F(1,22)=256, p=<0.0001)$ concentration-dependently inhibited the GABA-evoked ion current. These data demonstrate that both PFOS and PFOA exert antagonistic effects on the $\mathrm{GABA}_{\mathrm{A}}$ receptor. Notably, as shown in Fig. 1a, the PFOS-induced inhibition of the GABA-evoked ion current is not readily reversible. In contrast, the PFOA-induced inhibition is reversed within seconds (Fig. 1b). 

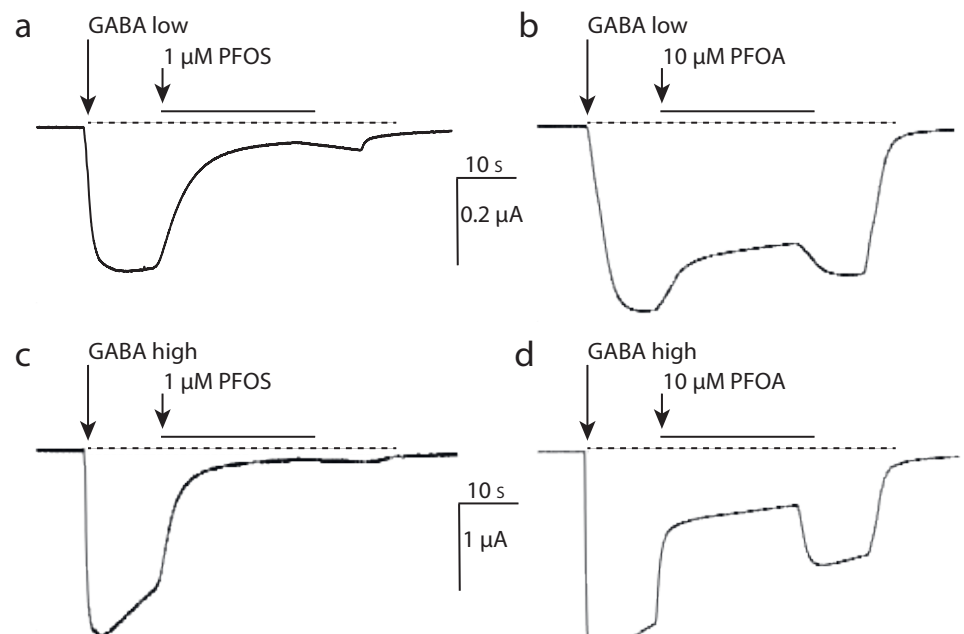

d GABA high
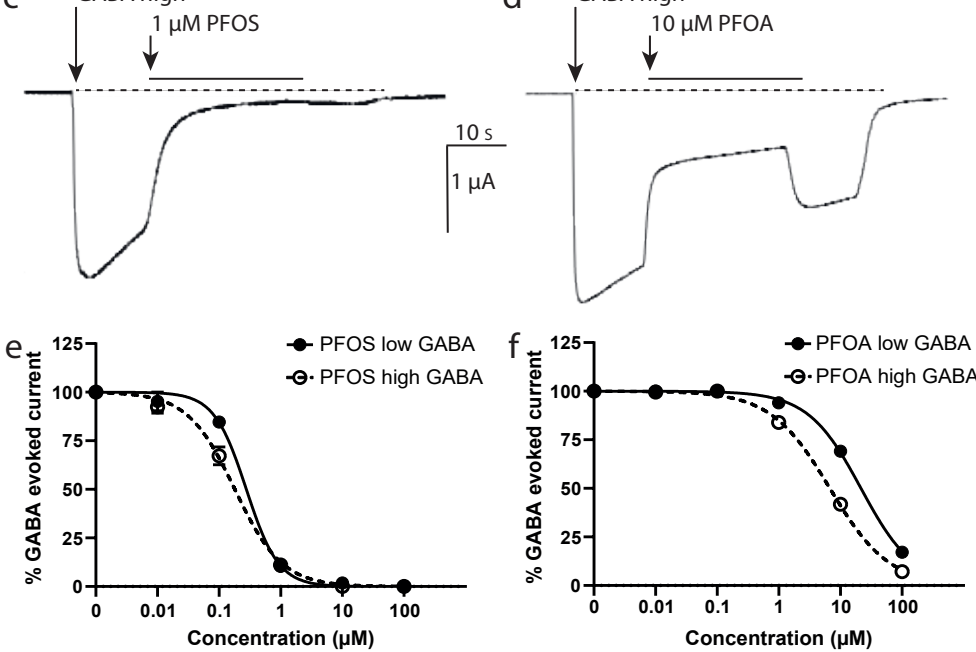

Figure 1. Antagonistic effects of PFOS and PFOA on the human $G_{A B A}$ receptor. Example recordings of inhibition of GABA-evoked ion currents by co-application of PFOS ( $1 \mu \mathrm{M}$, a and c) or PFOA (10 $\mu \mathrm{M}, \mathrm{b}$ and d) with GABA (at low and high effective GABA concentration, respectively). PFOS-induced inhibition is poorly reversible ( $a$ and $c$ ), whereas PFOA-induced inhibition is reversed within seconds ( $b$ and d). Scale bar applies to all traces. Concentration-response curves show the concentration-dependent inhibition of GABA-evoked responses by PFOS (e) and PFOA (f) on the human $\mathrm{GAB}_{\mathrm{A}}$ receptor with at low (e and f; solid line) and high (e and f; dashed line) effective GABA concentrations. Inhibition is presented as percentage of the GABA-evoked response (mean \pm SEM, $n=3-4$ oocytes per concentration from $\mathrm{N}=1-2$ batches). 
Table 1. Inhibition of GABA-evoked ion current by PFOS or PFOA. Mean percentage of inhibition of the current $( \pm$ SEM (n)) induced during exposure to PFOS or PFOA. Inhibitions were compared to control and p-values are given; n.s.: non-significant.

\begin{tabular}{|c|c|c|c|c|c|}
\hline & \multirow[b]{2}{*}{ Concentration $(\mu \mathrm{M})$} & \multicolumn{2}{|c|}{$\begin{array}{l}\text { Low effective GABA } \\
\text { concentration }\end{array}$} & \multicolumn{2}{|c|}{$\begin{array}{l}\text { High effective GABA } \\
\text { concentration }\end{array}$} \\
\hline & & \% Inhibition & $p$-value & \% Inhibition & $p$-value \\
\hline \multirow{5}{*}{$\begin{array}{l}\text { D } \\
\text { 㟔 } \\
\text { 2 }\end{array}$} & 0.01 & $4.8 \pm 4.8(4)$ & n.s & $7.6 \pm 3.3(4)$ & n.s \\
\hline & 0.1 & $15 \pm 1.8(4)$ & 0.002 & $33 \pm 4.6(4)$ & $<0.0001$ \\
\hline & 1 & $90 \pm 1.5(3)$ & $<0.0001$ & $89 \pm 0.9(3)$ & $<0.0001$ \\
\hline & 10 & $98 \pm 1.2(4)$ & $<0.0001$ & $100 \pm 0(3)$ & $<0.0001$ \\
\hline & 100 & $100 \pm 0(4)$ & $<0.0001$ & $100 \pm 0(4)$ & $<0.0001$ \\
\hline \multirow{5}{*}{ 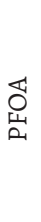 } & 0.01 & $0 \pm 0(4)$ & n.s. & $0.4 \pm 0.5(4)$ & n.s \\
\hline & 0.1 & $0 \pm 0(4)$ & n.s. & $0 \pm 0(3)$ & n.s \\
\hline & 1 & $6 \pm 0.4(4)$ & n.s & $16 \pm 0.4(4)$ & $<0.0001$ \\
\hline & 10 & $31 \pm 1.9(4)$ & $<0.0001$ & $58 \pm 1.5(4)$ & $<0.0001$ \\
\hline & 100 & $83 \pm 2.7(4)$ & $<0.0001$ & $93 \pm 2.7(4)$ & $<0.0001$ \\
\hline
\end{tabular}

Next, PFOS and PFOA were co-applied with a high concentration GABA (1 mM, $\mathrm{EC}_{100}$ ). PFOS induced a poorly reversible, concentration-dependent inhibition of the GABA-evoked ion current (LOEC $0.1 \mu \mathrm{M}, \mathrm{IC}_{50} 0.18 \mu \mathrm{M}, \mathrm{CI} 0.15-0.22$ $\mu \mathrm{M} ; \mathrm{F}(1,20)=10.4, p=0.004$; Fig. $1 \mathrm{C}$, e and Table 1 ), whereas the concentrationdependent PFOA-induced inhibition is rapidly reversed (LOEC $1 \mu \mathrm{M}, \mathrm{IC}_{50} 6.7 \mu \mathrm{M}$, CI $5.96-7.59 \mu \mathrm{M} ; \mathrm{F}(1,22)=66.2, p=<0.0001$; Fig. $1 \mathrm{~d}$, f and Table 1$)$.

The combined data indicate that PFOS and PFOA are potent, non-competitive $\mathrm{GABA}_{\mathrm{A}}$ receptor antagonists. As such, PFOS and PFOA may antagonize the inhibitory function of the CNS. To determine whether the effects of these PFAS also modulate neuronal network function, effects of PFOS and PFOA on spontaneous neuronal network activity were assessed.

\subsection{Effects of PFAS on rat cortical network activity}

Recently, non-invasive measurements of neuronal activity using MEA recordings have shown that rat primary cortical cultures develop spontaneous network activity (Robinette et al., 2011), which can be modulated by a diverse range of chemicals (McConnell et al., 2012; Nicolas et al., 2014; Dingemans et al., 2016; Vassallo et al., 2017). Currently, rat primary cortical neurons are the gold 
standard for MEA recordings. At DIV9, network bursts throughout the well can be observed. Therefore, we exposed rat primary cortical cultures at DIV9-11 to PFOS or PFOA to assess effects on spike, burst and network burst related parameters.

Acute exposure to PFOS or PFOA affected mean spike rate (MSR; Fig. 2a), with statistically significant differences between the concentrations (Welch's $\mathrm{F}(4,44.7)$ $=9.76, p=<0.0001$ for PFOS and Welch's $\mathrm{F}(4,46.5)=3.5, p=0.01$ for PFOA). Following exposure to $100 \mu \mathrm{M}$ PFOS, a marked increase in MSR could be observed $(175 \pm 13.1 \% ; p=<0.0001)$. PFOS also increased the mean burst rate (MBR; Fig. 2 b), with a statistically significant difference between concentrations (Welch's $F(4$, $46.0)=7.2, p=0.0001$ ). However, this increase was only significant compared to control following exposure to $100 \mu \mathrm{M}$ PFOS (175 $\pm 16.7 \% ; p=<0.0001)$. PFOS did not affect mean network burst rate (MNBR; Fig. 2c). PFOA only induced minor effects on spike and (network) burst rate. PFOA increased MNBR at $10 \mu \mathrm{M}$, but this increase was not significant. Neither PFOS nor PFOA affected burst duration (Fig. $2 \mathrm{~d}$ ), although there is a significant difference between groups for PFOA (Welch's $\mathrm{F}(4,45.8)=3.81, p=0.009$ ). Both PFAS did not affect network burst duration (Fig. 2e), but for PFOA the one-way Welch ANOVA was significant (Welch's $F(4,45.9$.) $=$ $3.67, p=0.011$ ). Effects observed at the highest test concentration are not a result of cytotoxicity (see supplemental data Fig. S2).

Overall, PFOS has a more profound effect on spontaneous neuronal network activity than PFOA. However, these data indicate that PFOS and PFOA induce only mild hyperexcitation in rat primary cortical cultures, which was unexpected considering the strong inhibition of human $\mathrm{GABA}_{\mathrm{A}}$ receptors. This apparent discrepancy may be the result of inter-species differences. To investigate whether this is the case, human induced pluripotent stem cell (hiPSC)-derived neurons can be used. It is known that there is variability between different hiPSC-derived neuronal cell lines (Little et al., 2019). This could be the result of the generation protocol or the maturation stage in which the neurons were frozen. To take these potential differences into account, two commercially available human induced pluripotent stem cell (hiPSC)-derived neuronal cell models were exposed to PFOS or PFOA: the iCell Glutaneuron - iCell Astrocytes co-culture and the SynFire iNS co-culture. 


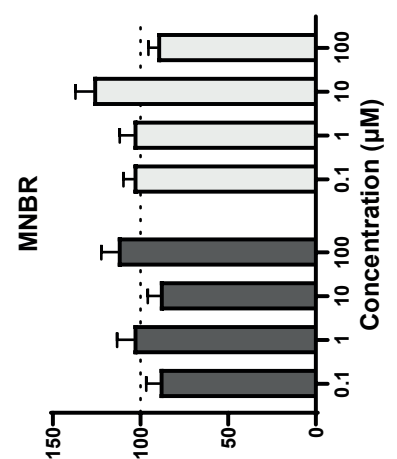

ןодиนоo s^ әбиечо \%
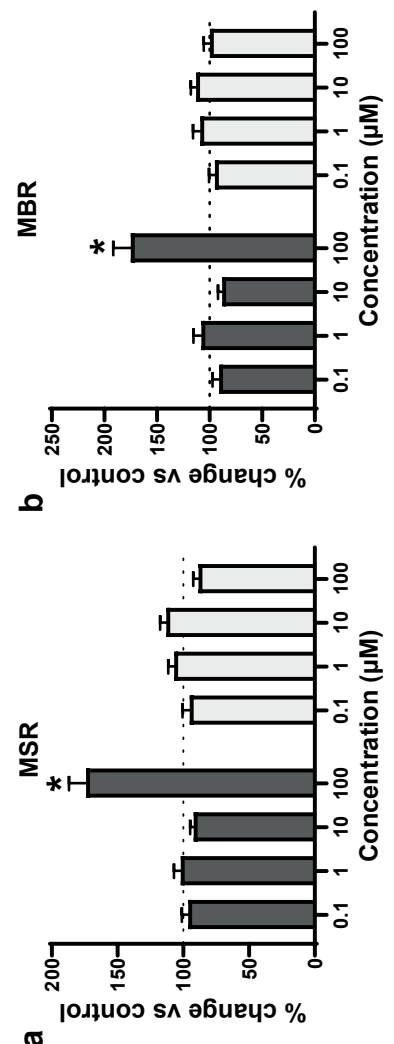

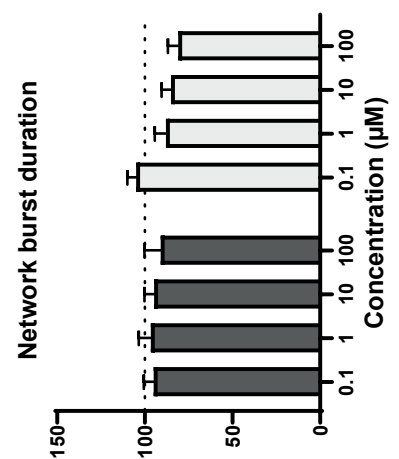

()

ןoגłuos sn әбuеyo \%

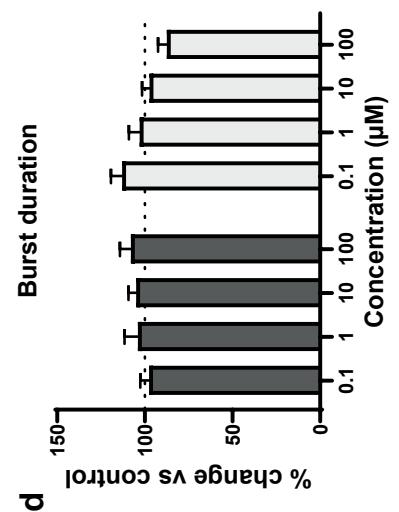




\subsection{Effects of PFAS on spontaneous hiPSC network activity}

Recent work in our laboratory has shown that hiPSC-derived neuronal co-cultures develop spontaneous activity and (network) bursting behaviour (Tukker et al., 2018). Acute exposure to PFOS (Welch's F $(4,34.9)=278, p=<0.0001$ ) and PFOA (Welch's $\mathrm{F}(4,32.9)=13, p=<0.0001$ ) affected MSR in the iCell Glutaneuron - iCell Astrocytes co-culture (Fig. 3a)). PFOS significantly decreased the MSR at $0.1 \mu \mathrm{M}$ (91.8 $\pm 3.18 \% ; p=0.04)$ and $100 \mu \mathrm{M}(8.2 \pm 3.71 \% ; p=<0.0001)$. For PFOA, MSR was significantly decreased at $1 \mu \mathrm{M}(88.9 \pm 2.64 ; p=0.02), 10 \mu \mathrm{M}(87.4 \pm 2.5 \% ; p$ $=0.02)$ and $100 \mu \mathrm{M}(59.7 \pm 6.49 ; p<0.0001)$ as compared to the control. Bursting was significantly affected following exposure to either PFAS (Welch's $\mathrm{F}(4,34.9)=$ $36.0, p<0.0001$ for PFOS and Welch's $\mathrm{F}(4,36.7)=6.67, p=0.0004$; Fig. 3b). PFOS exposure significantly decreased MBR at $100 \mu \mathrm{M}(9.43 \pm 4.2 \% ; p=<0.0001)$. For PFOA, MBR significantly decreased at $10 \mu \mathrm{M}(59.8 \pm 10.7 \% ; p=0.023)$ and 100 $\mu \mathrm{M}(49.5 \pm 8.73 \% ; p=0.0035)$. MNBR was decreased following exposure to PFOS (Welch's $\mathrm{F}(4,30.1)=51.0, p=<0.0001$ ) and PFOA (Welch's $\mathrm{F}(4,35.4)=15.6, p=<$ 0.0001 ; Fig. 3C). The decrease was significant following exposure to PFOS at 0.1 $\mu \mathrm{M}(54.8 \pm 9.1 \% ; p=0.002)$ and $100 \mu \mathrm{M}(2.58 \pm 1.9 \% ; p<0.0001)$ and for PFOA at $1 \mu \mathrm{M}(65.4 \pm 10.1 \% ; p=0.01), 10 \mu \mathrm{M}(44.7 \pm 9.03 ; p=0.0005)$ and $100 \mu \mathrm{M}(22.2 \pm$ 6.11; $p<0.0001)$. Exposure of the iCell Glutaneuron - iCell Astrocytes co-culture to either of the PFAS increased burst duration (Welch's $\mathrm{F}(4,24.0)=6.74, p=0.0009$ for PFOS and Welch's $\mathrm{F}(4,31.6)=6.71, p=0.0005$ for PFOA; Fig. 3d). The increase in burst duration following exposure to PFOS was significant at $10 \mu \mathrm{M}(206 \pm 39.3$; $p=0.002)$ and $100 \mu \mathrm{M}(245 \pm 41.3 \% ; p=0.0002)$. Following exposure to PFOA, the increase was significant at $1 \mu \mathrm{M}(194 \pm 18.9 \% ; p=<0.0001)$ and $100 \mu \mathrm{M}(174 \pm$ $30.6 ; p=0.01$ ). There was no significant effect on network burst duration (Fig. 3e), despite an apparent increase following exposure to $10 \mu \mathrm{M}$ PFOS. This change in activity pattern is further illustrated by spike raster plots that show the decrease in spiking and (network) bursting activity combined with prolonged (network) burst duration following exposure to $10 \mu \mathrm{M}$ PFOS (Fig. 4a) or $100 \mu \mathrm{M}$ PFOA (Fig. 4b).

Overall, effects are more pronounced following exposure to PFOS. However, PFOS and PFOA do not induce hyperexcitation in the iCell Glutaneuron - iCell Astrocytes co-culture model. Since the chemical sensitivity can differ between different hiPSC-derived neuronal cultures (Tukker et al., 2020), an additional hiPSC-derived cell model was tested, the SynFire iNS co-culture. 


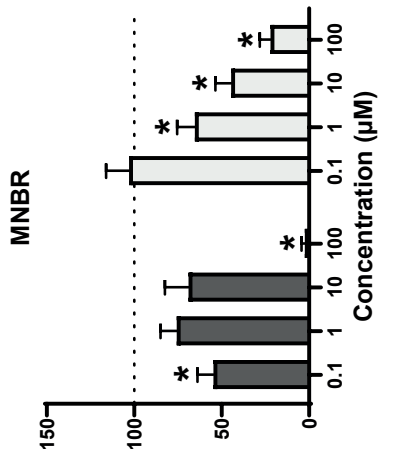

ןодио s^ әбиецว \%

0
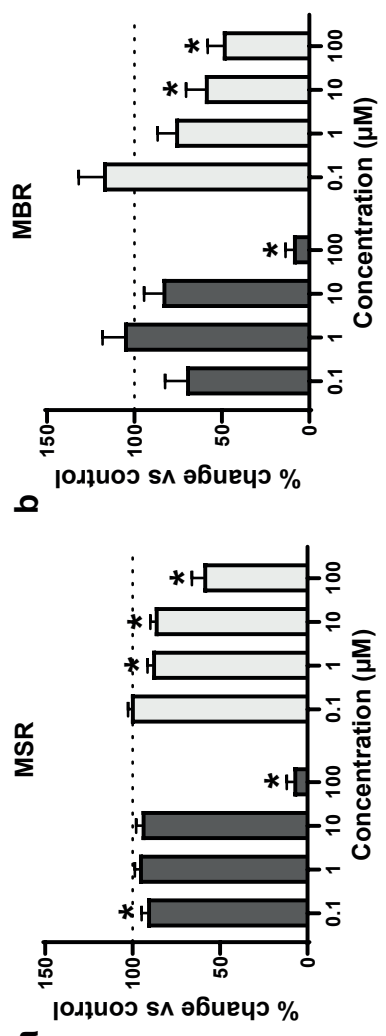

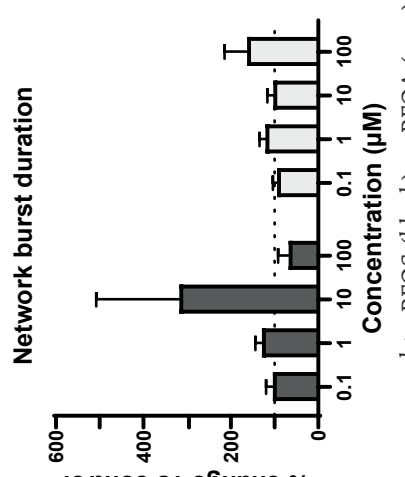

๘
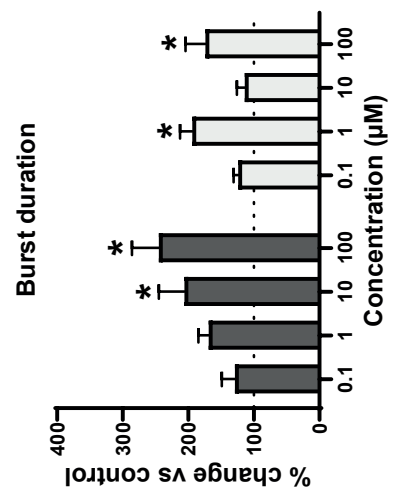

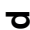

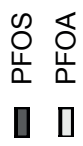



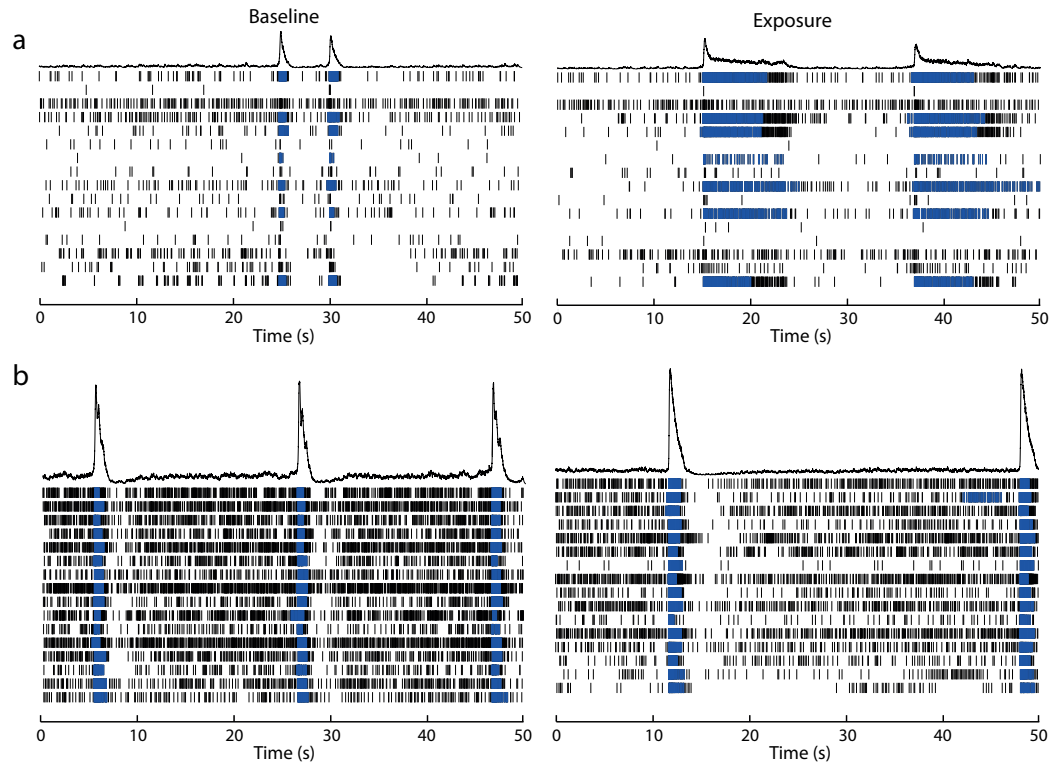

Figure 4. Spike raster plots illustrating the pattern of activity of a representative well of the iCell Glutaneuron - iCell Astrocytes co-culture before exposure (left) and the same well following exposure (right) to PFOS $10 \mu \mathrm{M}$ (a) or PFOA $100 \mu \mathrm{M}$ (b). Each row depicts one electrode in a well and each tick mark represents one spike in a $50 \mathrm{~s}$ interval. Spikes are depicted in black and bursts are depicted in blue.

The SynFire iNS co-culture contains a higher ratio of astrocytes compared to the iCell Glutaneuron - iCell Astrocytes co-culture and although the ratio glutamatergic to GABAergic neurons is comparable, the total cell number is much higher thus the number of glutamatergic neurons is also higher in this culture (Tukker et al., 2020). Therefore, the SynFire iNS co-culture is more active and excitable. Exposure of SynFire iNS co-culture to PFOS affected the MSR (Welch's $F(4,16.9)=3.18, \mathrm{p}=$ 0.04 for PFOS) with a significant decrease at $100 \mu \mathrm{M}$ (69.1 \pm 12 ; $\mathrm{p}=0.04$; Fig. 5a) as compared to control. Exposure to PFOA decreased the MSR at $100 \mu \mathrm{M}$, but this decrease was not significant. MBR was only significantly decreased by PFOS (Welch's $\mathrm{F}(4,18.2)=5.24, \mathrm{p}=0.006)$ at $100 \mu \mathrm{M}(58.3 \pm 8.13 ; \mathrm{p}=0.04$; Fig. 5b). Neither PFOS nor PFOA significantly disturbed MNBR (Fig. 5c). Burst duration and network burst duration were not significantly affected, although an increase could be observed following exposure to PFOS $100 \mu \mathrm{M}$ (Figs. 5d, e). 

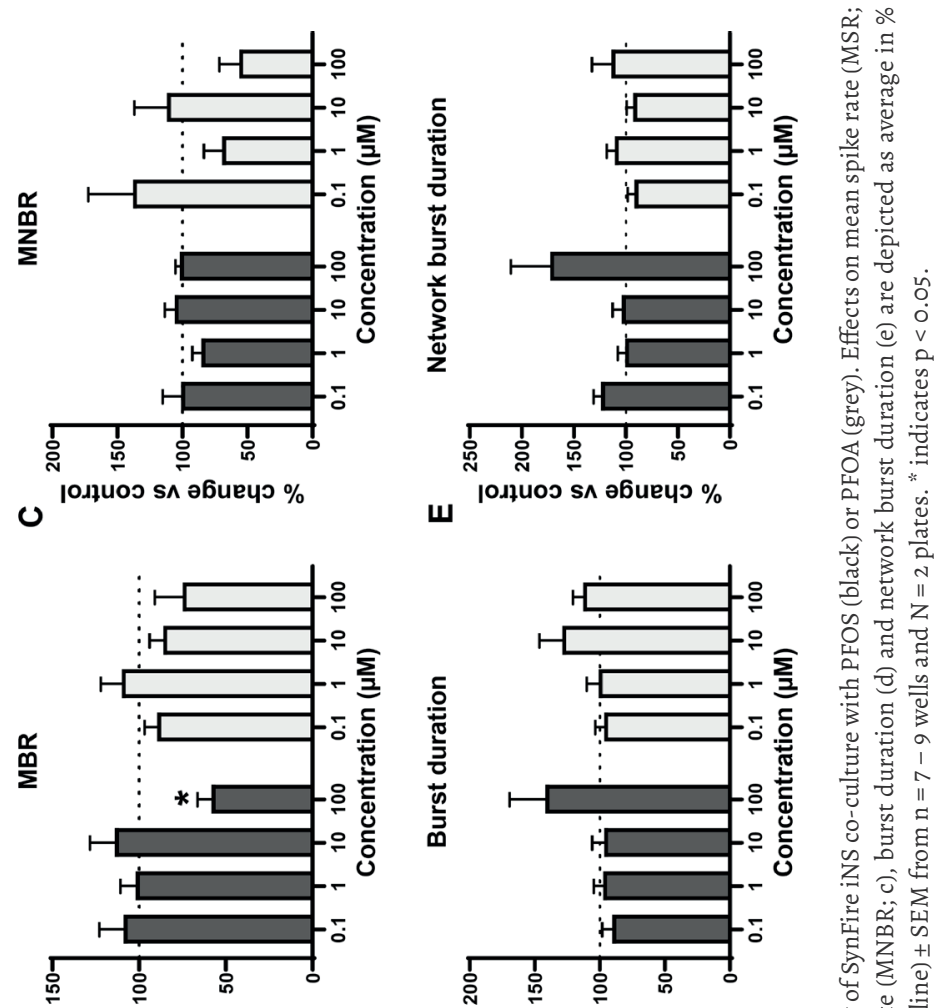

$m$ |одйоo sn әбиечо \%

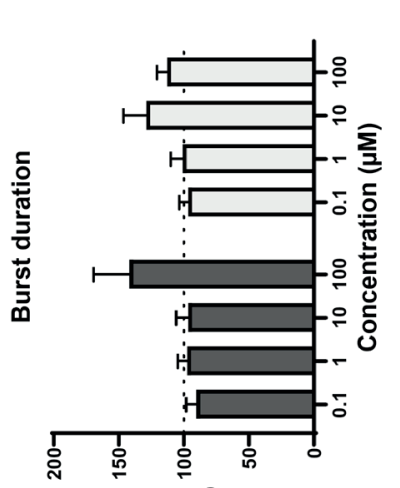

|од⿰ио s^ әбиецว \%
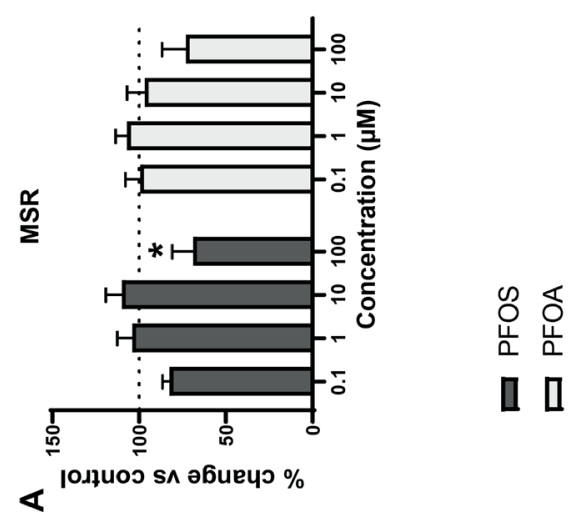


\subsection{Comparison of PFAS effects in different cell models}

LOECs for the activity metrics MSR, MBR and MNBR differ between the three different cell models (Table 2). The iCell Glutaneuron - iCell Astrocytes co-culture is the only model for which LOECs could be defined for PFOS and PFOA on all three metrics. This hiPSC-derived neuronal model is also the most sensitive of the three. The rat primary cortical neurons and SynFire iNS co-culture model show comparable LOECs, but respond differently to PFOS and PFOA exposure.

Table 2. LOECs of PFOS and PFOA for the different activity parameters (mean spike rate (MSR), mean burst rate (MBR) and mean network burst rate (MNBR)) on the different cell models. indicates no LOEC could be defined.

\begin{tabular}{lllllll} 
& \multicolumn{2}{c}{ MSR } & \multicolumn{2}{c}{ MBR } & \multicolumn{2}{c}{ MNBR } \\
& PFOS & PFOA & PFOS & PFOA & PFOS & PFOA \\
\hline $\begin{array}{l}\text { Rat primary cortical neurons } \\
\text { iCell Glutaneuron - iCellAstrocytes } \\
\text { co-culture }\end{array}$ & $100 \mu \mathrm{M}$ & - & $100 \mu \mathrm{M}$ & - & - & - \\
\begin{tabular}{l} 
SynFireins co-culture \\
\hline
\end{tabular} & $0.1 \mu \mathrm{M}$ & $1 \mu \mathrm{M}$ & $100 \mu \mathrm{M}$ & $10 \mu \mathrm{M}$ & $0.1 \mu \mathrm{M}$ & $1 \mu \mathrm{M}$ \\
\hline
\end{tabular}

To further illustrate the effect of PFOS and PFOA on the rat primary cortical neurons and the two hiPSC-derived neuronal models, we created a heatmap including 19 parameters that are descriptive for neuronal (network) activity (Fig. 6; Table 3). Although many changes (and some concentration-dependent changes) are visible in the heatmap and in Fig. 3, it should be noted that many of these changes do not reach statistical significance. Nevertheless, it is clear that the rat cortex is rather insensitive, though an increased activity can be observed following exposure to $100 \mu \mathrm{M}$. The iCell Glutaneuron - iCell Astrocytes coculture model is the most sensitive model. This can be seen from the profound decrease in MSR, MBR and MNBR and the increase in burst duration. The SynFire iNS co-culture is just as (in)sensitive as the rat primary cortical culture (Figs. 2, 5 and 6), though with an increase, in contrast to a decrease, in activity following exposure to $100 \mu \mathrm{M}$. It is also apparent that PFOS has a stronger and more excitatory effect than PFOA. In all three models, synchronicity parameters are least affected. Overall, this highlights that there are differences between human and rodent models. It also indicates that there are differences between the human model systems. Overall the model system used can to a large degree determine the type and degree of the effect. 


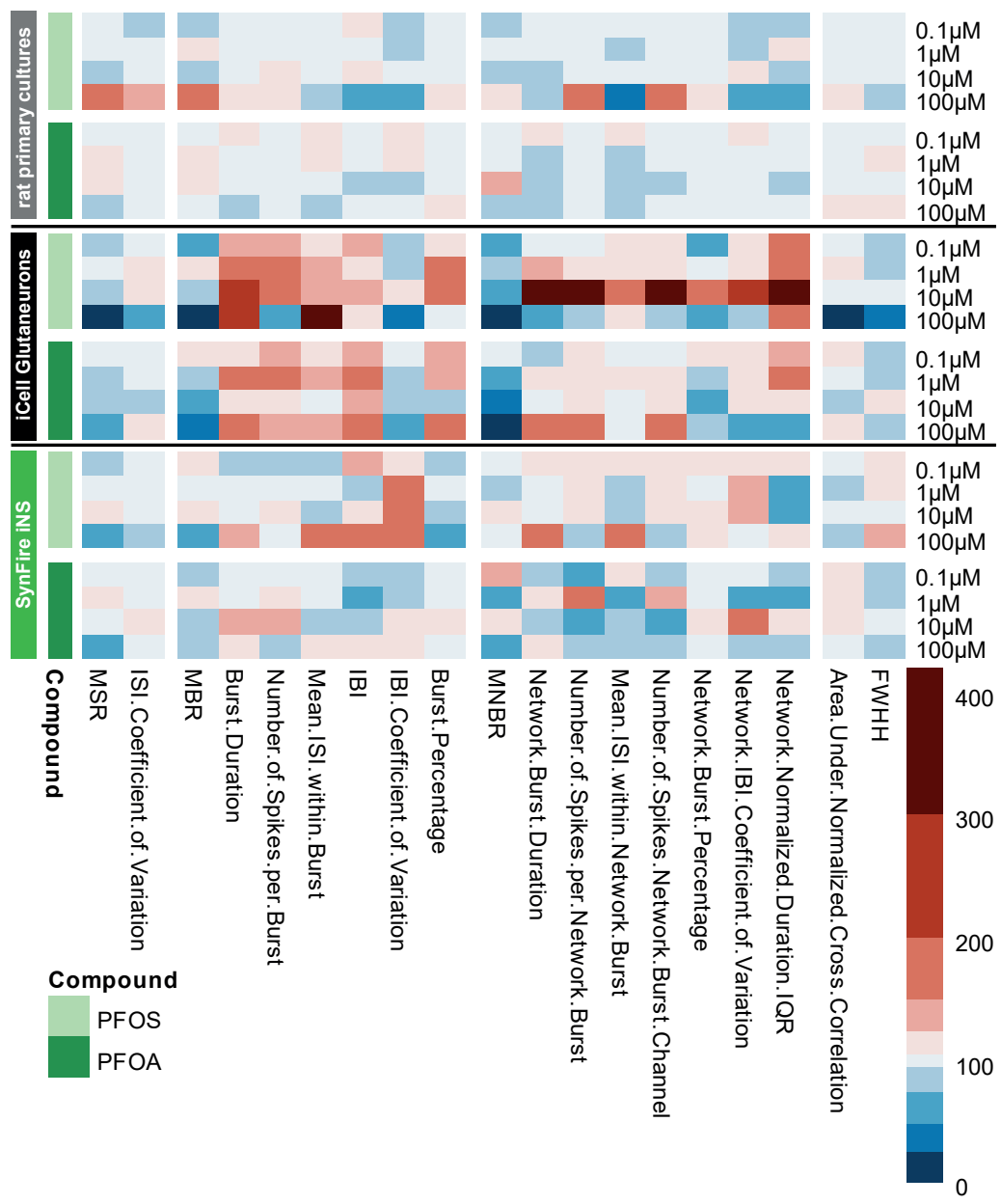

Figure 6. Heatmap of the effects of PFOS (light green) and PFOA (darker green) on selected metric parameters on rat primary cortical neurons (top; grey), iCell Glutaneuron - iCell Astrocytes (middle; black) and SynFire iNS co-culture (bottom; green). Colour scaling is based on the magnitude change in percentages relative to vehicle control based on $n=18-20$ wells and $\mathrm{N}=4$ plates for rat primary cortical neurons; $\mathrm{n}=3-17$ wells and $\mathrm{N}=3-5$ plates for iCell Glutaneuron - iCell Astrocytes; $\mathrm{n}=6-9$ wells $\mathrm{N}=2$ plates for SynFire iNS co-culture. 


\section{DISCUSSION}

PFOS and PFOA are omnipresent in the environment. In vivo and in vitro research have indicated the neurotoxic potential of both compounds (Mariussen, 2012). However, the underlying mechanisms of action are still debated. In the present study we show for the first time that PFOS and PFOA exhibit concentrationdependent antagonistic effects on the human $\alpha_{1} \beta_{2} \gamma_{2 L} G_{A B A}$ receptor at submicromolar concentrations (Fig. 1, Table 1). It is important to note that we tested only the most abundant $\alpha_{1} \beta_{2} \gamma_{2 L}$ receptor subunit combination. As many other $\mathrm{GABA}_{\mathrm{A}}$ receptor subtypes are present in the nervous system, PFAS may have different effects on these different receptor subtypes. The GABA-evoked current is rapidly restored once exposure to PFOA stops, but reversibility of the effect caused by PFOS is slow. This indicates that PFOA most likely has a lower receptor affinity than PFOS, which could be caused by the differences in head group. The reversibility could explain why at a high effective GABA concentration the LOEC of PFOA $(1 \mu \mathrm{M})$ is tenfold higher than the LOEC of PFOS $(0.1 \mu \mathrm{M})$. The observed inhibition of the GABA-evoked current occurs independently of the effective GABA concentration, implying that both PFAS are non-competitive GABA antagonists. This also indicates that neither PFOS nor PFOA binds to the GABA binding site located at the interface between the $\alpha$ and $\beta$ subunit, but are more likely to bind to modulatory binding sites. A variety of compounds, including convulsants and barbiturates, target these modulatory binding sites (D'Hulst et al., 2009). However, when positive or negative allosteric modulators bind to modulatory binding sites, the effect of the compound usually decreases with an increasing concentration of GABA. This is in contrast with our results. The agonistic effects of PFOS and PFOA could therefore be the result of a pore block through insertion of (a part of) the molecule into the receptor pore. This pore block would then prevent influx of chloride ions following binding of GABA to the GABA binding site. Full elucidation of the precise mode of action of PFAS on $\alpha_{1} \beta_{2} \gamma_{2 L} G A B A_{A}$ receptor function would require extensive mechanistic research, including measuring voltage-current curves in presence and absence of PFAS. Such experiments may elucidate whether PFOS and/ or PFOA cause a voltagedependent pore block, which is a likely mechanism of action given our results. However, regardless the exact molecular mechanism, the antagonistic effects of PFOS and PFOA on the human $\mathrm{GABA}_{\mathrm{A}}$ receptor can be hypothesized to cause hyperexcitation in neuronal networks. 
This hypothesis is further supported by evidence that PFOS induces the influx of extracellular calcium (Liao et al., 2008). However, when primary rat cortical neurons are exposed to PFAS, excitation is observed only in spike and burst activity at $100 \mu \mathrm{M}$ PFOS, whereas no significant excitatory effects of PFOA were found (Fig. 2 and 6). As there were no effects on primary rat cortical neurons at low or submicromolar levels of PFAS, as observed for human $\mathrm{GABA}_{\mathrm{A}}$ receptor, two different hiPSC-derived neuronal co-cultures were exposed to PFOS and PFOA to exclude inter-species differences. In contrast to the expected hyperexcitation, neither of the hiPSC-derived neuronal models showed hyperexcitation following acute exposure to PFOS or PFOA. In the iCell Glutaneurons - iCell Astrocytes co-culture, a strong inhibition of activity in combination with a prolonged burst duration was found following exposure to PFOS or PFOA (Fig. 3, 4 and 6). This model showed spike and network burst LOECs for PFOS (0.1 $\mu \mathrm{M})$ and PFOA $(1 \mu \mathrm{M})$ that were comparable to the human $\mathrm{GABA}_{\mathrm{A}}$ receptor studies. In contrast, in the SynFire iNS co-culture showed an apparent lack of sensitivity comparable to the rat primary cortical culture (Figs. 5 and 6). While the human iPSC models thus differ in sensitivity, they both show inhibition of neuronal activity in contrast to the rat primary culture. This hints towards a specific species-difference. However, giving the inhibitory effect on the human $\alpha_{1} \beta_{2} \gamma_{2 L} G A B A_{A}$ receptor, these findings also imply that PFAS may have additional targets that mask the inhibition of the $\mathrm{GABA}_{\mathrm{A}}$ receptor and/or that compensatory mechanisms are at play in neuronal networks to dampen the loss of $\mathrm{GABA}_{\mathrm{A}}$ receptor function. For example, PFAS could act on different neurotransmitter receptors, such as glutamate receptors, thereby masking the inhibition of the $\mathrm{GABA}_{\mathrm{A}}$ receptor. This idea is supported by research that showed that PFOS induces NMDA receptor mediated excitotoxicity (Berntsen et al., 2018). Toxicity results of a high influx of extracellular $\mathrm{Ca}^{2+}$, which in its turn over-activates the NMDA receptor. Although this study supports the argument that PFAS hit multiple targets, it should be noted that these results were obtained following $24 \mathrm{~h}$ exposure, as compared to our acute exposure study. Alternatively, the hyperexcitability effects of PFOS and PFOA may become apparent only after chronic exposure. These effects may then be the result of changes in for example the AMPA receptor composition or expression, as it has been previously shown that prolonged exposure of rat primary cortical neurons to $1 \mu \mathrm{M}$ PFOS lowers the expression of the AMPA receptor subunit GluR2, resulting in increased intracellular calcium concentrations and enhanced glutamate sensitivity(Ishida et al., 2017). It is also possible that the differences between the rodent and human neuronal model are due to an immature phenotype of the hiPSC-models. However, the used models have been shown to have a mature phenotype with 
hyperpolarizing GABA-responses and expression of mature transporters like KCC2 and NKCC (Cellular Dynamics International, n.d.; Neucyte Inc., 2019).

Compared to the two hiPCs-derived neuronal models, the rat primary cortical culture contains the highest ratio of astrocytes, about 45\% (Görtz et al., 2004; Tukker et al., 2016). Astrocytes increase the level of network activity (Tang et al., 2013; Ishii et al., 2017; Tukker et al., 2018) and modulate responses to toxicological insults (Wu, Yang, et al., 2017). It has been shown that astrocytes can also provide protection against adverse effects of PFOS on rat hippocampal neurons (Li et al., 2017). Therefore, the lack of an excitatory effect at lower concentrations could be due to the protective effect of the astrocytes present. When astrocytes are exposed for $24 \mathrm{~h}$ to concentrations higher than $50 \mu \mathrm{M}$ PFOS, astrotoxicity starts to occur, thereby increasing the risk on excitotoxicity (Li et al., 2017). Although exposure duration in our study was shorter and no cytotoxicity was observed, the increase in spike and burst activity following acute exposure of rat primary cortical neurons to a high PFOS concentration could be indicative of the onset of excitotoxicity. Another potential explanation for the inhibition of activity in the hiPSC-derived neuronal cultures could reside in the PFOS-induced shift in the activation curves of voltage-gated sodium channels towards hyperpolarization (Harada et al., 2006; Liao, Cui, et al., 2009), making it harder for the cell to reach an action potential. Future research to elucidate the possible counteracting mechanisms of action observed in our network study of PFOS and PFOA could focus on patch-clamp studies in neuronal networks following both acute and chronic exposure.

For PFOS and PFOA human serum to plasma ratios are 1:1(Ehresman et al., 2007). Reported plasma or serum concentrations for PFOA fall for the general population in the range between $0.001-0.2 \mu \mathrm{M}$ (Fromme et al., 2009; Wu, Sun, et al., 2017; Wang et al., 2018). In the occupationally exposed population, levels as high as $2.5 \mu \mathrm{M}$ are found (Ehresman et al., 2007). Thus, LOECs reported on $\mathrm{GABA}_{\mathrm{A}}$ receptor function, spike rate and network burst activity $(1 \mu \mathrm{M})$ are below this level. Importantly, PFOA can cross the blood-brain barrier (Wang et al., 2018). Therefore, PFOA could be a risk for the occupationally exposed population. PFOS can cross the blood-brain barrier less efficient than PFOA (Wang et al., 2018), but the LOECs reported here for PFOS are lower than for PFOA. Reported plasma or serum concentrations for PFOS fall in the range between $0.002-1.3$ $\mu \mathrm{M}$ in the general population (Fromme et al., 2009; Wu, Sun, et al., 2017; Wang et al., 2018) and in occupationally exposed workers the levels are even higher, 
ranging from 0.27 to $10 \mu \mathrm{M}$ (Ehresman et al., 2007; Fromme et al., 2009). LOECs on $\mathrm{GABA}_{\mathrm{A}}$ receptor data and on spike activity in an hiPSC-derived neuronal coculture reported in this study are within or below the range found in human plasma levels. It should be noted though that human exposure to PFAS is low dose, but continuous. Our in vitro study should therefore be considered mainly as hazard characterisation, highlighting the potential target(s) of PFOS and PFOA. Our findings, combined with the fact that PFAS can cross the blood-brain barrier, indicate that these compounds have a clear potential to cause neurotoxic effects in the occupationally exposed populations as well as in the general population.

\section{MATERIAL AND METHODS}

\subsection{Animals}

All experiments were conducted in accordance with Dutch law and approved by the Ethical Committee for Animal Experiments of Utrecht University. Animals were treated humanely and all efforts were made to alleviate suffering. Xenopus laevis oocytes were purchased from EcoCyte Bioscience (Castrop-Rauxel, Germany). Primary cultures of rat cortical neurons were prepared from postnatal day (PND) 0-1 Wistar rat pups (Envigo, Horst, The Netherlands).

\subsection{Chemicals}

$\mathrm{MgSO}_{4}, \mathrm{NaHCO}_{3}$ and $\mathrm{Ca}\left(\mathrm{NO}_{3}\right)$ were purchased from Merck (Darmstadt, Germany). Neurobasal-A medium, Dulbecco's Modified Eagle Medium (DMEM)-F12, penicillin - streptomycin $(5000 \mathrm{U} / \mathrm{mL}-5000 \mu \mathrm{g} / \mathrm{mL}$ for rat primary cortical culture media and $10.000 \mathrm{U} / \mathrm{mL}-10.000 \mu \mathrm{g} / \mathrm{mL}$ for hiPSC medium), B27 plus supplement, N2 supplement and L-glutamine were obtained from Life Technologies (Bleiswijk, The Netherlands). iCell Neural Supplement B and Nervous System Supplement were provided by Cellular Dynamics International (Madison, WI, USA). BrainPhys neuronal medium was obtained from StemCell Technologies (Cologne, Germany). SynFire seeding basal medium, short-term basal medium, long-term basal medium and accessory supplements were provided by NeuCyte (Sunnyvale, CA, USA). Laminin (L2020), 50\% polyethyleneimine (PEI) solution, sodium borate, boric acid and all other chemicals (unless stated otherwise) were obtained from Sigma-Aldrich (Zwijndrecht, The Netherlands). 
Stock solutions of PFOS (>98\% purity, Fluka, Zwijndrecht, The Netherlands) and PFOA (>96\% purity, Acros Organics, Geel, Belgium) were made in purity-checked dimethyl sulfoxide (DMSO) and stored at $4^{\circ} \mathrm{C}$. Final concentration of DMSO in PFOS- or PFOA-containing solutions was always kept below $0.1 \%$ (vol/vol). DMSO at concentrations up to $0.5 \%(\mathrm{vol} / \mathrm{vol})$ had no effect on $\mathrm{GABA}_{\mathrm{A}}$ receptormediated currents.

\section{$4.3 \alpha_{1} \beta_{2} \gamma_{2 L}$ GABA $A_{A}$ receptor expression in Xenopus laevis oocytes}

All procedures have been described previously (Hendriks et al., 2010; Hondebrink et al., 2015). Briefly, complementary DNA (cDNA) coding for the human $\alpha_{1}, \beta_{2}$ (Origene, Rockville, USA) and human $\gamma_{2 \mathrm{~L}}$ subunits (kindly provided by Dr Paul J. Whiting, Merck Sharp \& Dohme Research Laboratories, Neuroscience Research Center, Harlow, Essex, U.K.) was dissolved in distilled water at a 1:1:1 molar ratio and injected ( $23 \mathrm{~nL} /$ oocyte, $\sim 1 \mathrm{ng}$ of each subunit) into the nuclei of stage $\mathrm{V}$ or VI oocytes using a Nanoject Automatic Oocyte Injector (Drummond, Broomall, PA). Following injection, oocytes were incubated at $21^{\circ} \mathrm{C}$ in modified Barth's solution containing (in $\mathrm{mM}$ ) $88 \mathrm{NaCl}$, I KCl, $2.4 \mathrm{NaHCO}_{3}, 0.3 \mathrm{Ca}\left(\mathrm{NO}_{3}\right)_{2}, 0.4 \mathrm{CaCl}_{2}, 0.82$ $\mathrm{MgSO}_{4}, 15 \mathrm{HEPES}$, and $10 \mu \mathrm{g} / \mathrm{mL}$ neomycin (pH 7.6 with $\mathrm{NaOH}$ ).

\subsection{Electrophysiological recordings of $G A B A_{A}$ receptor currents}

Electrophysiological recordings were performed on oocytes following 4-6 days of incubation to ensure sufficient translation of injected cDNA and functional expression of $\alpha_{1} \beta_{2} \gamma_{2 \mathrm{~L}} \mathrm{GABA}_{\mathrm{A}}$ receptors in the membrane. Ion currents associated with $\mathrm{GABA}_{\mathrm{A}}$ receptor activity were measured with the two-electrode voltageclamp technique using a Gene Clamp 500B amplifier (Axon Instruments, Union City, CA) with high-voltage output stage as described previously (Hendriks et al., 2010; Hondebrink et al., 2015). Recording microelectrodes (0.1-1 $M \Omega$ ) were filled with $\mathrm{KCl}(3 \mathrm{M})$. Oocytes, placed in a custom-built Teflon oocyte recording chamber, were voltage-clamped at $-60 \mathrm{mV}$ and continuously superfused $(\sim 30 \mathrm{~mL} /$ $\mathrm{min}$ ) with saline solution containing (in $\mathrm{mM}$ ) $1 \mathrm{CaCl}_{2}, 10 \mathrm{HEPES}$ ( $\mathrm{pH} 7.2$ with $\mathrm{NaOH}$ ), $2.5 \mathrm{KCl}, 115 \mathrm{NaCl}$. Membrane currents were low-pass filtered (8-pole Bessel; $3 \mathrm{~dB}$ at $0.3 \mathrm{kHz}$ ), digitized (12 bits; 1024 samples per record), and recorded on disk using WinWCP (Strathclyde Electrophysiological Software, Glasgow, Scotland) for further analysis.

Aliquots of freshly thawed GABA stock solution in distilled water and PFOS or PFOA in DMSO were added to the saline immediately before the experiments. GABA-responsive oocytes were exposed to saline, saline containing GABA (34 $\mu \mathrm{M}$ 
or $1 \mathrm{mM}$ ), saline containing a PFAS or saline containing GABA in combination with a PFAS by switching the perfusate using a servomotor-operated valve. To determine whether oocytes were GABA responsive, oocytes were super-fused for $40 \mathrm{~s}$ with a saline solution containing GABA. If oocytes were responsive, they were exposed for $10 \mathrm{~s}$ to saline containing GABA, followed directly by a $20 \mathrm{~s}$ exposure of saline containing GABA and PFAS and finally to $10 \mathrm{~s}$ of saline containing GABA. Concentrations of $0.01-100 \mu \mathrm{M}$ PFOS or PFOA were chosen to overlap with earlier in vitro studies and extended to include human serum and plasma concentrations. A washout period of 4 min between each application was introduced, allowing receptors to recover from desensitization. Sham-injected oocytes did not show any ion current upon superfusion with GABA, PFOS, or PFOA at concentrations tested, alone or in co-application with GABA (data not shown). To minimize adsorption of PFOS or PFOA to the perfusion system, glass reservoirs and Teflon tubes (polytetrafluoroethylene, Rubber, Hilversum, The Netherlands) were used.

\subsection{Cell culture}

Primary rat cortical cultures and hiPSC-derived neuronal co-cultures were kept at $37^{\circ} \mathrm{C}$ in a humidified $5 \% \mathrm{CO}_{2}$ incubator. All cell culture surface materials were pre-coated with $0.1 \%$ PEI solution diluted in borate buffer ( $24 \mathrm{mM}$ sodium borate/50 $\mathrm{mM}$ boric acid in Milli-Q adjusted to $\mathrm{pH}$ 8.4).

Primary rat cortical cells were isolated from PNDO-1 Wistar rat pups as described previously (Dingemans et al., 2016; Tukker et al., 2016) with minor modifications. Briefly, PNDO-1 pups were decapitated and cortices were rapidly dissected on ice and kept in serum free dissection medium (Neurobasal-A supplemented with 25 $\mathrm{g} / \mathrm{L}$ sucrose, $450 \mu \mathrm{M}$ L-glutamine, $30 \mu \mathrm{M}$ glutamate, $1 \%$ penicillin/streptomycin and $2 \%$ B27 plus supplement, $\mathrm{pH} 7.4$ ) during the entire procedure. Cortices were dissociated to a single-cell suspension by mincing with scissors, trituration and filtering through a $100 \mu \mathrm{m}$ mesh (EASYstrainer, Greiner). The cell suspension was diluted to a $2 \times 10^{6}$ cells $/ \mathrm{mL}$ solution. Droplets of $50 \mu \mathrm{L}$ were placed on the electrode fields in wells of pre-coated 48-well MEA plates (Axion BioSystems Inc., Atlanta, GA, USA). Cells were left to adhere for $\sim 2 \mathrm{hr}$ before adding $450 \mu \mathrm{L}$ serum free dissection medium. At DIV4, $90 \%$ of the serum free dissection medium was replaced with serum free culture medium (Neurobasal-A supplemented with 25 g/L sucrose, $450 \mu \mathrm{M}$ L-glutamine, $1 \%$ penicillin/streptomycin and $2 \%$ B27 plus supplement, pH 7.4). Rat primary cortical neurons were exposed at DIV9-11. 
iCell Glutaneurons (Lot\# 103288, containing 70\% glutamatergic and $~ 30 \%$ GABAergic neurons); Cellular Dynamics International, Madison, WI, USA) and iCell Astrocytes (Lot\# 103956; Cellular Dynamics International, Madison, WI, USA) were thawed and cultured according to manufacturer's protocol. In short, each cell type was thawed separately in supplemented BrainPhys medium (BrainPhys medium supplemented with 2\% iCell Neural Supplement B, 1\% Nervous System Supplement, $1 \% \mathrm{~N} 2,1 \%$ penicillin - streptomycin and $0.1 \%$ laminin). Following centrifugation, the cell pellet was diluted in dotting medium (supplemented BrainPhys medium with $10 \%$ laminin) till $15 \times 10^{3}$ cells/ $\mu \mathrm{L}$ for iCell Glutaneurons and $6.7 \times 10^{3} \mathrm{cells} / \mu \mathrm{L}$ for iCell Astrocytes. Before plating, iCell Glutaneurons and iCell Astrocytes were premixed into a co-culture containing $120 \times 10^{3}$ iCell Glutaneurons and $20 \times 10^{3}$ iCell Astrocytes. Cells were plated in $11 \mu \mathrm{L}$ droplets (140 x $10^{3}$ cells/droplet with $85 \%$ iCell Glutaneurons and 15\% iCell Astrocytes) over the electrode field of pre-coated 48-well MEA plates. After plating, cells were allowed to adhere for $\sim 1 \mathrm{hr}$ following which $300 \mu \mathrm{L}$ of room temperature (RT) supplemented BrainPhys medium was added. 50\% Medium changes with RT supplemented BrainPhys medium took place at DIV1, 2, 4, 6, 8, 10, 12 and 14. The iCell Glutaneurons - iCell Astrocytes cultures were exposed at DIV14.

SynFire glutamatergic neurons (Lot\# 118B and 000124), SynFire GABAergic neurons (Lot\# 118B and 000124) and SynFire astrocytes (Lot\# 13029; all from NeuCyte Sunnyvalle, CA, USA) were thawed and cultured according to manufacturer's protocol. Briefly, each cell type was thawed separately in DMEM-F12. Each cell pellet was dissolved in complete seeding medium (containing the seeding supplement) till a density of $10 \times 10^{3} \mathrm{cells} / \mu \mathrm{L}$ following which a mixture was made containing $52 \%$ glutamatergic neurons, $22 \%$ GABAergic neurons and $26 \%$ astrocytes. This mixture was plated in $50 \mu \mathrm{L}$ droplets $\left(270 \times 10^{3}\right.$ cells per droplet) over the electrode field of pre-coated MEA plates. Cells were placed in the incubator and left overnight to adhere. The next day, $250 \mu \mathrm{L} /$ well of complete short-term maintenance medium (containing the supplement) was added. At DIV3 and 5, 50\% medium changes with short-term maintenance medium took place, after which culture medium was gradually replaced by complete long-term maintenance medium (containing supplements A and B) through 50\% medium changes at DIV7, 10, 13, 16, 19, 22, 25 and 25. SynFire iNS co-cultures were exposed at DIV28. 


\subsection{MEA measurements}

Each well of a 48-well MEA plate contains 16 nanotextured gold micro-electrodes $(\sim 40-50 \mu \mathrm{m}$ diameter; $350 \mu \mathrm{m}$ spacing) with 4 integrated ground electrodes yielding a total of 768 channels for simultaneous recording (for review see Johnstone et al., 2010). Spontaneous electrical activity was recorded as described previously (Nicolas et al., 2014; Tukker et al., 2016). Briefly, signals were recorded at the day of experiments (DIV9-11 for rat primary cortical cultures, DIV14 for iCell Glutaneurons - iCell Astrocytes co-cultures and DIV28 for SynFire iNS cocultures) using a Maestro 768-channel amplifier with integrated heating system and temperature controller and a data acquisition interface (Axion BioSystems Inc., Atlanta, GA, USA). Data acquisition was managed with Axion's Integrated Studio (AxIS 2.4.2.13) and recorded as .RAW files. All channels were sampled simultaneously with a gain of $1200 \mathrm{x}$ and a sampling frequency of $12.5 \mathrm{kHz} /$ channel with a 200-5000 Hz band-pass filter. Prior to the recording, MEA plates were allowed to equilibrate for 5-10 min in the Maestro.

In order to determine effect of reference compounds on spontaneous neuronal activity (spiking and (network) bursting behaviour) on the three different cell cultures, a $30 \mathrm{~min}$ baseline recording was made. Following this baseline recording, wells were exposed (10 x dilution for primary rat cortical cultures and SynFire iNS co-culture and $30 \mathrm{x}$ dilution for iCell Glutaneurons - iCell Astrocytes co-culture) to PFOS, PFOA or the solvent control and directly a 30 min exposure recording was made. In order to overlap with earlier in vitro experiments and to fall in the range of reported human serum and plasma levels of PFOS and PFOA, concentrations of $0.1-100 \mu \mathrm{M}$ were tested. Stock solutions of PFOS and PFOA in DMSO were diluted in culture medium to obtain desired concentrations. In all experiments, the solvent concentration never exceeded $0.1 \% \mathrm{v} / \mathrm{v}$. In order to prevent receptor (de)sensitization each well was exposed to a single concentration. For each experimental condition, MEA plates from at least two different plating rounds were used.

\subsection{Data analysis and statistics}

Peak amplitudes of GABA-evoked ion currents were measured and normalized to the maximal amplitude (at $1 \mathrm{mM}$ ) of GABA-evoked control responses to adjust for differences in receptor expression levels among oocytes and for small variations in response amplitudes over time as described previously (Hendriks et al., 2010; Hondebrink et al., 2015). Normalized ion currents were plotted against GABA concentration in each experiment. GABA concentration-effect curves were fitted 
to the data obtained in separate experiments using Prism (Graphpad Software, La Jolla, CA, USA). The percentage of PFOS- or PFOA-induced inhibition of the GABA-evoked ion current was calculated from the quotient of the maximum amplitude of the GABA-PFOS/PFOA co-application response and the maximum amplitude of the control (GABA) response.

To determine (modulation of) spontaneous activity, .RAW data files were rerecorded to obtain Alpha Map files. In this re-recording, spikes were detected with the AxIS spike detector (Adaptive threshold crossing, Ada BandFIt v2) using a variable threshold spike detector set at $7 \mathrm{x}$ (rat primary cortical cultures) or $5.5 \mathrm{x}$ (hiPSC cultures) standard deviation (SD) of internal noise level (rms) on each electrode. Post/pre-spike duration was set to 3.6/2.4 ms respectively. For further data analysis, spike files were loaded in NeuralMetric Tool (version 2.2.4, Axion BioSystems) and only active electrodes (MSR $\geq 6$ spikes/min) in active wells ( $\geq 1$ active electrode) were included. Since (network) bursting behaviour is crucial for in vivo neuronal communication(Izhikevich et al., 2003), it was analysed using the Poisson Surprise method(Legéndy and Salcman, 1985) with a minimal surprise of 10 and a minimum bursting frequency of 0.005 bursts/sec. An adaptive threshold algorithm was used for the extraction of network bursts.

The effects of PFOS and PFOA on spontaneous neuronal activity were determined by comparing the baseline activity with activity following exposure. A custom-made MS Excel macro was used to calculate treatment ratios (TR) per well for the different metric parameters (Table 3) by: (parameter $r_{\text {exposure }} /$ parameter $_{\text {baseline }}$ ) x 100\%. Hereafter, TRs were normalized to appropriate vehicle control (DMSO). To prevent inclusion of exposure artefacts, effect analysis was performed 20-30 min post-exposure.

Oocytes or wells that showed effect two times SD above or below average were considered outliers and removed for further data analysis (1.1\% for oocytes, $4.2 \%$ for rat primary cortical neurons, $4.7 \%$ for iCell Glutaneuron - iCell Astrocytes cocultures and 3.6\% for SynFire iNS co-cultures). For oocyte data, the concentrationdependent effects of PFOS and PFOA were determined by one-way ANOVA and a post hoc Bonferroni test. Best-fit concentration-response curves were plotted in PRISM (version 8.0.1) by nonlinear regression with a four-parameter variable slope with the bottom value constrained to 0 and top value to 100 to improve the fit. In the case of MEA data, concentration-dependent effects were determined by a one-way Welch ANOVA and a post hoc Dunnett test. P-values $<0.05$ were considered statistically significant. All statistical analyses were performed in $\mathrm{R}$ 
version 3.6.0 (R core team 2019) with base R or by using the DescTools package (by Signorell et al., 2019, version 0.99.28).

Data are presented as mean \pm standard error of the mean (SEM) from the number of oocytes or wells (n) indicated, derived from 1 to 2 independent batches (N) of oocytes, 2 plating rounds $(\mathrm{N})$ for the rat primary cortical culture, 5 plating rounds (N) for the iCell Glutaneuron - iCell Astrocytes co-culture and 2 independent plating rounds $(\mathrm{N})$ for the SynFire iNS co-culture unless stated otherwise. IC50 values are reported with confidence intervals (CI). LOECs are defined as lowest statistically significant concentrations. 
Table 3. Description of different metric parameters. Adapted from(Tukker et al., 2020)

\section{Metric parameter}

Mean spike rate (MSR)

ISI coefficient of variation

Mean burst rate (MBR)

Burst duration

Number of spikes per burst

Mean inter-spike interval (ISI) within burst

Inter-burst interval (IBI)

IBI coefficient of variation

Burst percentage

Mean network burst rate (MNBR)

Network burst duration

Number of spikes per network burst

Mean ISI within network burst

Number of spikes per network burst per channel

Network burst percentage

Network IBI coefficient of variation

Network normalized duration IQR

Area under normalized cross-correlation

Full width at half height (FWHH) of normalized cross-correlation

\section{Description}

Total number of spikes divided by recording time $(\mathrm{Hz})$

Standard deviation ISI (time between spikes) divided by the mean ISI. Measure for spike regularity: 0 indicates perfect spike distribution, >1 signals bursting Total number of bursts divided by recording time $(\mathrm{Hz})$ Average time from the first spike in a burst till the last spike (s)

Average number of spikes occurring in a burst

Mean inter-spike interval within a burst (s)

Time between the last spike of a burst and the first spike of a subsequent burst (s)

Standard deviation of IBI divided by the mean IBI. Measure for burst regularity

Percentage of total number of spikes occurring in a burst Total number of network bursts divided by recording time $(\mathrm{Hz})$

Average time from the first spike till the last spike in a network burst (s)

Average number of spikes occurring in a network burst Average of the mean ISIs within a network burst (s) Average number of spikes across the network bursts in the network burst divided by the number of electrodes participating in the network burst

Percentage of total spikes occurring in a network burst Standard deviation of network IBI divided by the mean network IBI. Measure of network burst rhythmicity: value is small when bursts occur at regular interval and increases when bursts occur more sporadic Interquartile range of network bursts durations. Measure for network burst duration regularity: larger values indicate wide variation in duration.

Area under inter-electrode cross-correlation normalized to the auto-correlations. The higher the value, the greater the synchronicity of the network

Width at half height of the normalized crosscorrelogram. Measure for network synchrony: the higher the value, the less synchronized the network is 


\section{DATA AVAILABILITY}

The datasets generated during and/or analysed during the current study are available from the corresponding author on reasonable request.

\section{ACKNOWLEDGEMENTS}

This work was funded by a grant from the National Centre for the Replacement, Refinement and Reduction of Animals in Research ( $\mathrm{NC}_{3} \mathrm{Rs}$; project number 50308-372160). Members of the Neurotoxicology Research Group are gratefully acknowledged for helpful discussions. We thank Fiona Wijnolts for her assistance with cell culture work and Barbara Vreede (University library, Utrecht University) for her help with R scripts.

\section{AUTHOR CONTRIBUTIONS}

All authors contributed to the study conception and design. Material preparation, data collection and analysis were performed by AT, LB and GK. The first draft of the manuscript was written by AT and $\mathrm{HH}$. RW and JL were involved with study design and helpful discussion concerning the results and manuscript. All authors commented on previous versions of the manuscript. All authors read and approved the final manuscript.

\section{CONFLICT OF INTEREST}

The authors declare no competing interests. 


\section{SUPPLEMENTAL DATA}

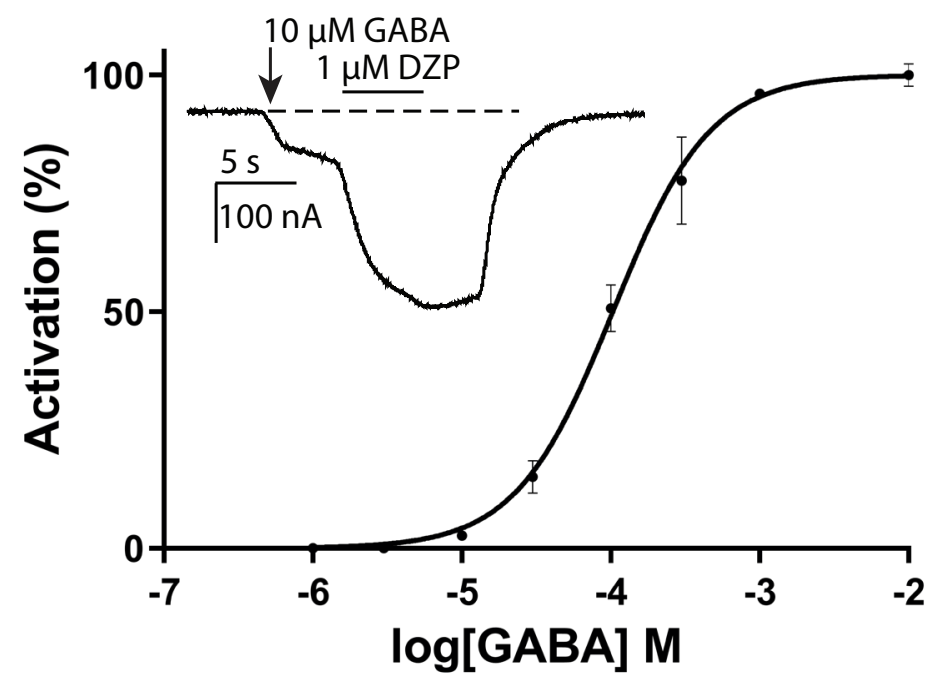

Figure S1. Concentration-effect curve of the GABA-evoked ion current in oocytes expressing the $\alpha_{1} \beta_{2} \gamma_{2 L}$ subunits forming the GABA receptor. Curve is fitted based on mean values $( \pm S D$ ) from $4-16$ oocytes. Co-exposure of GABA and $1 \mu \mathrm{M}$ diazepam (DZP) evokes a potentiation confirming the presence of a functional $\gamma_{2 L}$ subunit (insert). 


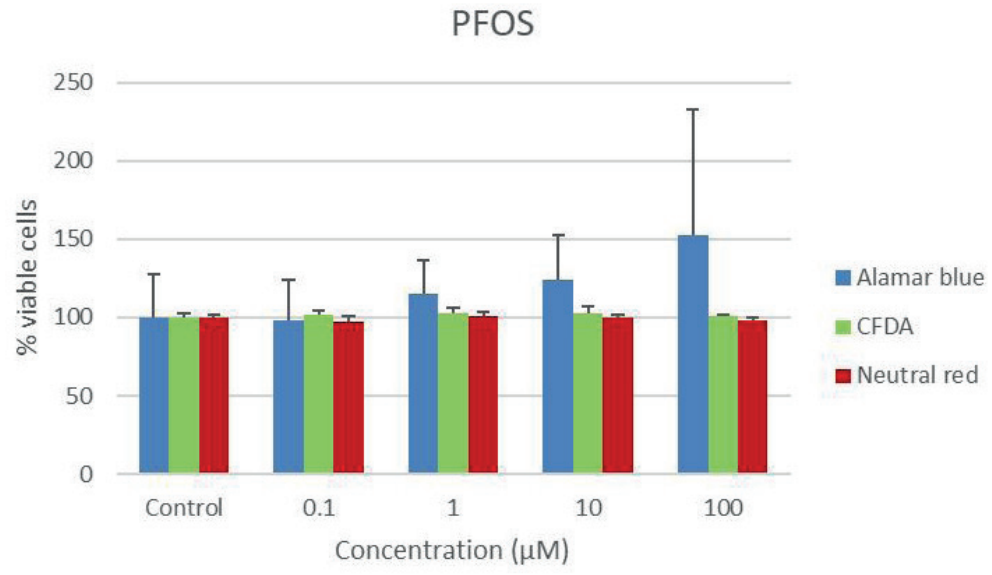

\section{PFOA}

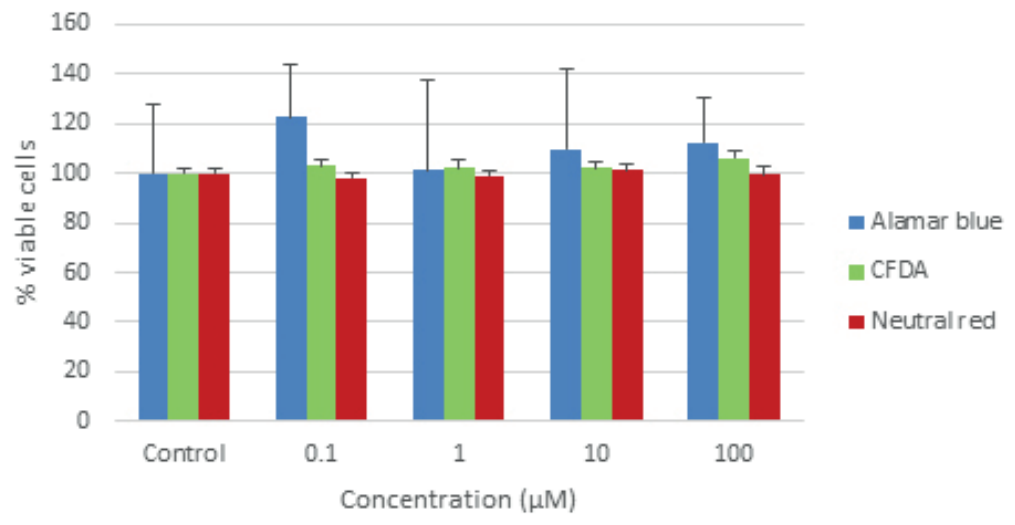

Figure S2. Cytotoxicity data of PFOS (top) and PFOA (bottom) from an alamar blue (blue), CFDA (green) and neutral red (red) assay measured $24 \mathrm{~h}$ after a 30 min exposure of rat primary cortical cultures. No cytotoxicity can be observed. $\mathrm{n}=6-12$ wells, $\mathrm{N}=2$ plates. Data is presented as mean $\pm \mathrm{SD}$. 


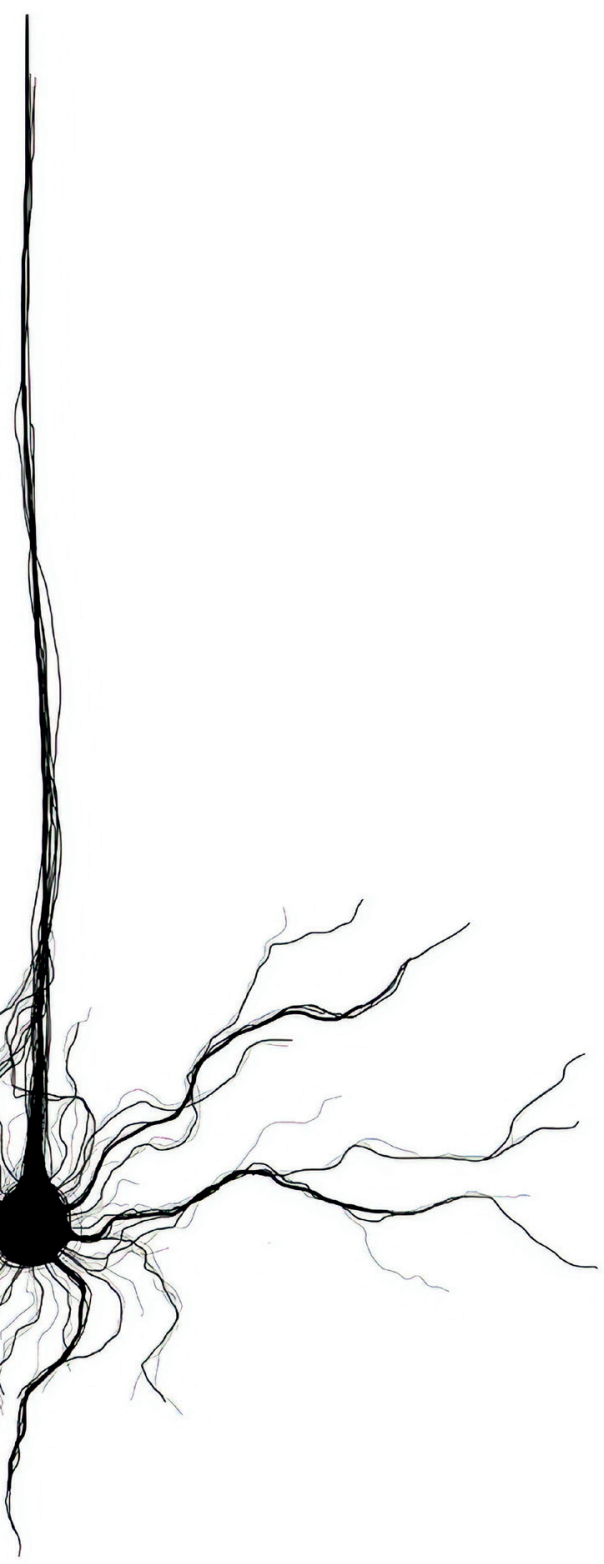


Part III

\section{HUMAN IPSC-DERIVED \\ NEURONAL MODELS}


Chapter 5

Is the time right for in vitro neurotoxicity testing using human iPSC-derived neurons?

Anke M. Tukker*, , , Martje W.G.D.M. de Groot", , Fiona M.J. Wijnolts', Emma E.J. Kasteel $^{1}$, Laura Hondebrink ${ }^{2}$ and Remco H.S. Westerink ${ }^{1}$

* Both authors contributed equally to this study

I Neurotoxicology Research Group, Toxicology Division, Institute for Risk Assessment Sciences (IRAS), Faculty of Veterinary Medicine, Utrecht University, P.O. Box 80.177, NL-3508 TD Utrecht, The Netherlands.

2 National Poisons Information Center (NVIC), University Medical Center Utrecht, P.O. Box 85500, NL-3508 GA, Utrecht, The Netherlands.

ALTEX (2016), volume 33, issue 3, pp. $261-71$

DOI: 10.14573/altex.1510091

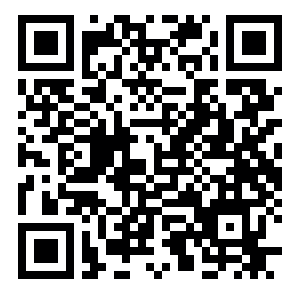




\section{ABSTRACT}

Current neurotoxicity testing heavily relies on expensive, time consuming and ethically debated in vivo animal experiments that are unsuitable for screening large number of chemicals. Consequently, there is a clear need for (high-throughput) in vitro test strategies, preferably using human cells as this increases relevance and eliminates the need for interspecies translation. However, human stem cell-derived neurons used to date are not well characterised, require prolonged differentiation and are potentially subject to batch-to-batch variation, ethical concerns and country-specific legislations. Recently, a number of human induced pluripotent stem cell (iPSC)-derived neurons became commercially available that may circumvent these concerns.

We therefore used immunofluorescent stainings to demonstrate that human iPSC-derived neurons from various suppliers form mixed neuronal cultures, consisting of different types of (excitatory and inhibitory) neurons. Using multiwell micro-electrode array (mwMEA) recordings, we demonstrate that these human iPSC-derived cultures develop spontaneous neuronal activity over time, which can be modulated by different physiological, toxicological and pharmacological compounds. Additional single cell calcium imaging illustrates the presence of functional GABA, glutamate, and acetylcholine receptors as well as voltage-gated calcium channels.

While human iPSC-derived neuronal cultures appear not yet suitable to fully replace the rat primary cortical model, our data indicate that these rapidly differentiating, commercially available human iPSC-derived neuronal cultures are already suitable for in vitro prioritisation and effect screening studies. Further characterisation and toxicological validation is now required to facilitate acceptance and large-scale implementation of these animal-free, physiologically-relevant human iPSC-based models for future neurotoxicity testing.

Keywords: In vitro neurotoxicity screening, micro-electrode array (MEA), human induced pluripotent stem cell-derived neurons, mixed neuronal cultures, alternatives to animal testing 


\section{INTRODUCTION}

Current neurotoxicity testing heavily relies on animal experiments. These in vivo experiments are not only ethically debated, but are also expensive and time consuming and therefore unsuitable for screening large numbers of chemicals (Bal-Price et al., 2008). As a result, there is a clear need for the development of innovative (high-throughput) in vitro test strategies. Such in vitro strategies not only reduce animal use, but also increase mechanistic insight in the modes of action of the tested chemicals (Bal-Price et al., 2008; Llorens et al., 2012; Van Thriel et al., 2012). However, in vitro neurotoxicity testing receives considerable criticism and scepticism, in particular from a regulatory perspective, as it has been suggested that in vitro models are too simplified to mimic the complexity of the in vivo brain and are not predictive enough. However, these concerns seem unjustified if the in vitro models used and endpoints assessed meet some defined basic requirements (Westerink, 2013).

In vitro screening models should represent the in vivo situation as closely as possible. For neurotoxicity testing this basically translates into an in vitro model that forms functional neuronal networks consisting of a mixed population of multiple types of (excitatory and inhibitory) neurons as well as supportive cells (e.g., astrocytes) to capture the complexity of the brain. The cellular morphology and heterogeneity of the formed neuronal networks can be assessed using immunofluorescent staining of specific proteins to identify the presence of astrocytes and (excitatory and inhibitory) neurons. While subsequent electrophysiological assessment of neuronal function is ideally suited to investigate chemically-induced changes in neuronal function and transmission (de Groot et al., 2013), it is often too endpoint-specific, requires training and expertise, and lacks the required throughput.

The recent introduction of micro-electrode array (MEA) recordings bypasses these limitations. MEAs consist of a cell culture surface with an integrated array of micro-electrodes that allows for the simultaneous and non-invasive recordings of (extracellular) local field potentials at different locations in an in vitro neuronal network at millisecond time scale (for review see Johnstone et al., 2010). Neuronal networks grown on MEAs possess many characteristics of neurons in vivo, including (the development of) spontaneous activity (Robinette et al., 2011) and responsiveness to neurotransmitters and pharmacological agents (Gross et al., 1997; for review, see Johnstone et al., 2010; de Groot et al., 2013). As such, neuronal networks on MEAs have been proposed as an in vitro neurotoxicity screening method that shows 
consistent reproducibility and reliability across different laboratories (Novellino et al., 2011) as well as high sensitivity and specificity (McConnell et al., 2012; Nicolas et al., 2014; Valdivia et al., 2014).

While many different in vitro models can be cultured on MEAs, primary rat cortical cultures are the current standard (Hogberg et al., 2011; McConnell et al., 2012; Valdivia et al., 2014). These primary cultures are well characterised, widely accepted, easily cultured and recapitulate many aspects of nervous system function (for review, see Johnstone et al., 2010; de Groot et al., 2013). However, as also outlined in NC3Rs' Neuratect Crack-it Challenge (www.NC3Rs.org.uk), the use of human neurons for in vitro neurotoxicity testing is preferred. Nevertheless, the use of human neurons for MEA recordings has been limited (Heikkilä et al., 2009; Ylä-Outinen, 2010; Kapucu et al., 2012), while there is no indication that human-derived neuronal networks will not exhibit the same properties as primary rat cortical cultures. Importantly, interspecies translation is no longer necessary if human-derived (stem) cells are used to assess chemically-induced effects on proliferation, migration and differentiation as well as other endpoints relevant for human (developmental) neurotoxicity (for review, see de Groot et al., 2013). However, human stem cell-derived neurons used to date are less well characterised than cortical neurons and potentially subject to batch-to-batch variation, which could hamper data interpretation. Moreover, these human stem cell-derived neurons require extensive ( $>3$ weeks) and costly differentiation on the MEA, making them less amenable to screening large numbers of chemicals in an efficient and timely manner. Finally, human stem cell-derived neurons may not be readily available for use in all laboratories as a result of restrictions due to ethical concerns and country-specific legislations. In recent years, a considerable number of human induced pluripotent stem cell (iPSC)-derived neurons became commercially available that may circumvent these difficulties. The potential of these human iPSC-derived neurons for in vitro neurotoxicity testing is however not yet firmly established.

We therefore purchased human iPSC-derived neurons from various commercial suppliers to demonstrate their mixed neuronal nature using immunofluorescent staining. Importantly, by characterising both the functional presence of neurotransmitter receptors and ion channels using single cell calcium imaging as well as the development and modulation of spontaneous neuronal network activity using mwMEA recordings we demonstrate their potential for future high-throughput neurotoxicity testing. Upon further characterisation and 
toxicological validation, the use of such physiologically-relevant human in vitro iPSC-based models can facilitate predictions of toxicological profiles and improve human risk assessment, while reducing or even replacing animal experiments for neurotoxicity testing.

\section{MATERIALS AND METHODS}

\subsection{Chemicals}

Neurobasa ${ }^{\circledR}$-A Medium, DMEM with high glucose, 1-glutamine, foetal bovine serum (FBS), N2 supplement, KnockOut Serum Replacement, Natural Mouse Laminin, penicillin-streptomycin (10000 U/mL-10000 $\mathrm{g} / \mathrm{mL}$ ), GlutaMAX, Geltrex, B27 supplement (without vitamin A) and Fura-2 AM were purchased from Life Technologies (Bleiswijk, The Netherlands). iCell Neurons Maintenance Medium (NRM-100-121-001) and iCell Neurons Medium Supplement (NRM-100-031-001) were purchased from Cellular Dynamics international (Madison, WI, USA). PreCoat Solution (GSM-9450), NeuralQ ${ }^{\mathrm{TM}}$ Basal Medium (GSM-9420) and GS21 ${ }^{\mathrm{TM}}$ Neural Supplement (GSM-3100) were purchased from MTI-GlobalStem (Gaithersburg, MD, USA). GlutaGRO was purchased from Corning Incorporated (Corning, NY, USA). DOPA. $4 \mathrm{U}^{\circ}$ Thawing Medium, DOPA. $4 \mathrm{U}^{\circ}$ Culture Medium and DA-supplement were purchased from Axiogenesis (Cologne, Germany). dl-Amphetamine was obtained from Spruyt Hillen (IJsselstein, The Netherlands). Poly-l-Ornithine (PLO), $50 \%$ polyethyleneimine-coated (PEI) solution, laminin, Sodium Borate, Boric Acid and all other chemicals (unless otherwise described) were purchased from SigmaAldrich (Zwijndrecht, The Netherlands).

Saline solution for $\mathrm{Ca}^{2+}$ imaging experiments (containing (in $\mathrm{mM}$ ) $125 \mathrm{NaCl}, 5.5 \mathrm{KCl}$, $2 \mathrm{CaCl}_{2}, 0.8 \mathrm{MgCl}_{2}, 10 \mathrm{HEPES}, 24$ glucose, and 36.5 sucrose) and high-potassium saline solution (containing (in mM) $5.5 \mathrm{NaCl}, 100 \mathrm{KCl}, 2 \mathrm{CaCl}_{2}, 0.8 \mathrm{MgCl}_{2}, 10 \mathrm{HEPES}$, 24 glucose and 36.5 sucrose) were prepared with deionized water (Milli- $Q$; resistivity; $10 \mathrm{M} \Omega \cdot \mathrm{cm}$ ). For both saline solutions, $\mathrm{pH}$ was set at 7.3. Saline solutions containing gamma-aminobutyric acid (GABA $100 \mu \mathrm{M})$, acetylcholine chloride (ACh $100 \mu \mathrm{M})$ or sodium L-glutamic acid (glutamate $100 \mu \mathrm{M}$ ) were prepared in separate aliquots for every experimental day and kept at $-20^{\circ} \mathrm{C}$ until use. 


\subsection{Cell culture}

Cells were cultured at $37^{\circ} \mathrm{C}$ in a humidified $5 \% \mathrm{CO}_{2}$ atmosphere according to supplier instructions. Briefly, iCell Neurons (NRC-100-010-001, Cellular Dynamics international, Madison, WI, USA) were seeded in Complete iCell Neurons Maintenance Medium (with $2 \%$ iCell Neurons Medium Supplement) supplemented with laminin $(10 \mu \mathrm{g} / \mathrm{mL})$. For immunofluorescent stainings, iCell ${ }^{\circ}$ Neurons were seeded at a density of 100.000 cells/chamber on Poly-l-Ornithine-coated ([PLO] $0.01 \%$ ) 8-chamber coverslips (Ibidi GmbH, Planegg, Germany). For MEA experiments, 70.000 cells/well were seeded as a $4 \mu \mathrm{L}$ droplet of cell suspension directly over the electrode field of each well of a 48-well MEA plate (Axion Biosystems Inc., Atlanta, USA) coated with $0.1 \%$ polyethyleneimine ([PEI] solution diluted in borate buffer [24 $\mathrm{mM}$ Sodium Borate $/ 50 \mathrm{mM}$ Boric Acid in Milli-Q adjusted to $\mathrm{pH}$ 8.4]). The droplet of cell suspension was allowed to adhere to the electrode field for $40 \mathrm{~min}$, after which $300 \mu \mathrm{L}$ of Complete iCell Maintenance Medium supplemented with laminin was added to each well. For $\mathrm{Ca}^{2+}$ imaging experiments, cells were seeded at a density of 175.000 cells/dish as a $300 \mu \mathrm{L}$ droplet on the glass of PEI-coated glass-bottom dishes (MatTek, Ashland, MA). The droplet of cell suspension was allowed to adhere to the glass for $40 \mathrm{~min}$ after which $1.5 \mathrm{~mL}$ of Complete iCell Maintenance Medium supplemented with laminin was added. After one to three days in vitro (DIV1 to 3), $50 \%$ of the medium in the coverslips was replaced by Complete Maintenance Medium, and $100 \%$ of the medium in the mwMEA plates and glass-bottom dishes was replaced by Neurobasal ${ }^{\circledR}-\mathrm{A}$ (NBA) medium supplemented with $10 \%$ KnockOut Serum Replacement, $100 \mathrm{U} / \mathrm{mL}$ penicillin and $100 \mu \mathrm{g} / \mathrm{mL}$ streptomycin. Every 3-4 days, $50 \%$ of the medium was refreshed: in coverslips by Complete Maintenance Medium and in the mwMEA plates and glass-bottom dishes by supplemented NBA medium. For iCell $^{\circ}$ Neurons $/ \mathrm{CDI}$ iCell ${ }^{\circ}$ Astrocytes co-cultures, iCell ${ }^{\circ}$ Neurons were seeded as described above. For immunofluorescent stainings, iCell ${ }^{\circ}$ neurons were seeded at a density of 66.000 cells/chamber in $150 \mu \mathrm{L}$ Complete Maintenance Medium supplemented with laminin on PLO-coated coverslips. iCell neurons were allowed to adhere for $40 \mathrm{~min}$, after which iCell ${ }^{\circ}$ Astrocytes (ASC-100-020-001-PT, Cellular Dynamics international, Madison, USA), thawed in DMEM with high glucose + $10 \%$ foetal bovine serum (FBS) and 1\% N2 supplement, were seeded at a density of 22.000 cells/chamber in $150 \mu \mathrm{L}$ laminin-supplemented Complete Maintenance Medium in each chamber on the coverslip. Co-cultures were refreshed as described above for the CDI iCell Neurons. HIP neurons (GSC-4312, MTI-GlobalStem, Gaithersburg, MD, USA) were seeded in Neuronal Maintenance Medium (NeuralQ ${ }^{\mathrm{TM}}$ Basal Medium supplemented with $2 \% \mathrm{GS}_{2} \mathrm{1}^{\mathrm{TM}}$ Neural Supplement and $2 \mathrm{mM}$ GlutaGRO). For immunofluorescent stainings 100.000 cells/chamber were seeded 
on coverslips and for MEA measurements, 90.000 cells/well were seeded as a $4 \mu \mathrm{L}$ droplet directly over the electrode field of each MEA well. Cells were allowed to attach for $20 \mathrm{~min}$ at room temperature (rt) and a subsequent $30 \mathrm{~min}$ in a humidified incubator at $37^{\circ} \mathrm{C}$ and $5 \% \mathrm{CO}_{2}$ after which $300 \mu \mathrm{L}$ Neuronal Maintenance Medium was added to each well. On DIV1 and every 3-4 days thereafter, $50 \%$ of the Neuronal Maintenance Medium was replaced by fresh Neuronal Maintenance Medium. All culture materials were coated with 1x GS Pre-Coat solution and Natural Mouse Laminin (15 $\mu \mathrm{g} / \mathrm{mL})$. DOPA. $4 U^{\circ}$ neurons (Axiogenesis, Cologne, Germany) were thawed in DOPA. $4 \mathrm{U}^{\circ}$ Thawing Medium supplemented with laminin $(10 \mu \mathrm{g} / \mathrm{mL})$ and seeded in DOPA. $4 \mathrm{U}^{\circ}$ Culture Medium (supplemented with 2.5\% DA-supplement and laminin $(10 \mu \mathrm{g} / \mathrm{mL})$ ). For immunofluorescent stainings, 52.000 cells/chamber were seeded on coverslips. For MEA experiments, 40.000 cells/well were seeded as a $5 \mu \mathrm{L}$ droplet directly over to the electrode field of the MEA wells. Cells were allowed to adhere to the electrode field for 1 hour, after which $300 \mu \mathrm{L}$ of laminin-supplemented DOPA. $4 \mathrm{U}^{\circ}$ Culture Medium was added to each well. Medium was refreshed every 2-3 days with DOPA. $4 \mathrm{U}^{\circ}$ Culture Medium. All culture materials were coated with Geltrex. For DOPA. $4 U^{\circ}$ neurons $/ i C e l l^{\circ}$ Astrocytes co-cultures, DOPA. $4 \mathrm{U}^{\circ}$ neurons were seeded as described above. For immunofluorescent stainings, 48.000 cells/chamber were seeded on coverslips in $150 \mu \mathrm{L}$ DOPA. $4 \mathrm{U}^{\circ}$ Culture Medium supplemented with laminin. Cells were allowed to adhere to the coverslips for 1 hour, after which $150 \mu \mathrm{L}$ of iCell Astrocytes, thawed in DMEM with high glucose $+10 \%$ FBS and $1 \%$ N2 supplement, were added in a density of 20.000 cells/chamber in lamininsupplemented DOPA. $4 \mathrm{U}^{\circ}$ Culture Medium. Medium was refreshed every 2-3 days with DOPA. $4 \mathrm{U}^{\circ}$ Culture Medium. All culture materials were coated with Geltrex. Primary rat cortical cultures were isolated from post-natal day (PND)1 Wistar rat pups in accordance with the Dutch law and approved by the Ethical Committee for Animal Experimentation of Utrecht University. Animals were treated humanely and efforts for alleviation of suffering were taken. Culture preparation was done as described previously (Nicolas et al., 2014; De Groot et al., 2016). Briefly, PND1 rat pups were decapitated and cortices were rapidly isolated on ice and kept in dissection medium (Neurobasal-A supplemented with sucrose [25 g/l], L-glutamine [450 $\mu \mathrm{M}]$, glutamate $[30 \mu \mathrm{M}]$, penicillin/streptomycin [1\%], and $\mathrm{FBS}[10 \%], \mathrm{pH} 7.4)$ during the entire isolation. Cells were seeded in dissection medium on poly-L-lysine $(50 \mu \mathrm{g} / \mathrm{mL})$ coated culture materials following which cells were kept at $37^{\circ} \mathrm{C}$ in a $5 \% \mathrm{CO}_{2}$ atmosphere. For MEA experiments, 100.000 cells/well were seeded as a $50 \mu \mathrm{L}$ droplet directly over the electrode field of the MEA wells. Cells were allowed to adhere for 2 hours, after which $450 \mu \mathrm{L}$ of dissection medium was added to each well. At DIV1, $90 \%$ of the dissection medium was replaced with glutamate medium (Neurobasal-A supplemented with 
sucrose $[25 \mathrm{~g} / \mathrm{L}], \mathrm{L}$-glutamine $[450 \mu \mathrm{M}]$, glutamate [30 $\mu \mathrm{M}]$, penicillin/streptomycin [1\%], and B-27 supplement [2\%], pH 7.4). At DIV4 and DIV11, $90 \%$ of the culture medium was replaced with FBS medium (Neurobasal-A supplemented with sucrose [25 g/L], L-glutamine [450 $\mathrm{MM}$ ], penicillin/streptomycin [1\%], and FBS [10\%], pH 7.4).

\subsection{Immunocytochemistry}

Different human iPSC-derived neurons were fixed on DIV8-14 with $4 \%$ paraformaldehyde (PFA) in $0.1 \mathrm{M}$ phosphate buffer ( $\mathrm{pH}$ 7.4) for $15 \mathrm{~min}$ at room temperature (RT). Subsequently, coverslips were quenched for PFA, permeabilised, and incubated with blocking buffer ( $2 \%$ bovine serum albumin and $0.1 \%$ saponin in PBS) containing $20 \mathrm{mM} \mathrm{NH} 4 \mathrm{Cl}$ for $20 \mathrm{~min}$ at RT. Each of the subsequent wash and incubation steps was performed in blocking buffer. Next, coverslips were incubated overnight at $4^{\circ} \mathrm{C}$ with rabbit anti- $\beta I I I$ tubulin (ab18207, Abcam, Cambridge, United Kingdom) at a final dilution of 1:500, goat anti-GFAP (ab53554, Abcam, Cambridge, United Kingdom) at a final dilution of 1:100, mouse anti-vGAT (131 001, Synaptic Systems, Göttingen, Germany) at a final dilution of 1:1000, and/or rabbit anti-vGluT1 (ab104898, Abcam, Cambridge, United Kingdom) at a final concentration of 1:1500. Subsequently, coverslips were washed 3 times with blocking buffer and incubated with donkey anti-rabbit Alexa 488, donkey anti-goat 594, donkey anti-mouse Alexa 488, and/or donkey anti-rabbit 594 (Life Technologies, Bleiswijk, The Netherlands) at a final dilution of 1:100 for $30 \mathrm{~min}$ at $\mathrm{rt}$ in the dark. Nuclear staining was performed by incubating the coverslips with 4',6-diamidino-2-phenylindole (DAPI; Life Technologies, Bleiswjik, The Netherlands) at a concentration of $200 \mathrm{nM}$ for 2-3 min at $\mathrm{rt}$ in the dark. The washing procedure was repeated and coverslips were sealed with FluorSave (Calbiochem, San Diego, California). Immunostained coverslips were visualised using a Leica SPEII Confocal microscope (Leica DMI4000 equipped with TCS SPE-II) using a $\times 20$ oil immersion objective (N.A. 1.4-0.7) and images were captured as *.tif files using Leica Application Suite Advanced Fluorescence software (LAS AF version 2.6.0; Leica Microsystems GmbH, Wetzlar, Germany).

\subsection{MEA measurements}

Different human iPSC-derived neurons were cultured on 48-well MEA plates, with each well containing an electrode array of 16 nanotextured gold micro-electrodes $(\sim 40-50 \mu \mathrm{m}$ diameter; $350 \mu \mathrm{m}$ centre-to-centre spacing) with 4 integrated ground electrodes, yielding a total of 768 channels that can be recorded simultaneously (see Figure I for illustration). Spontaneous electrical activity is recorded as described previously (de Groot et al., 2014; Nicolas et al., 2014; De Groot et al., 2016). Briefly, signals were recorded at various culture durations (DIV4-9) using a Maestro 
768-channel amplifier with integrated heating system and temperature controller and a data acquisition interface (Axion Biosystems Inc.). Axion's Integrated Studio (AxIS 2.0.2.11) was used to manage data acquisition. Prior to the $30 \mathrm{~min}$ recording of spontaneous activity, MEA plates were allowed to equilibrate in the Maestro for $\sim 5$ min. To obtain raw data files, channels were sampled simultaneously at a constant temperature of $37^{\circ} \mathrm{C}$ with a gain of $1200 \times$ and a sampling frequency of $12.5 \mathrm{kHz} /$ channel using a band-pass filter $(200-5000 \mathrm{~Hz})$.

To determine changes in spontaneous neuronal activity of iCell neurons (without added astrocytes), activity was recorded for $30 \mathrm{~min}$ at DIV7-8. Immediately following this baseline recording, cells were exposed ( $33 \mu \mathrm{L} /$ well, i.e. 1:10 dilution) to test compounds or appropriate (solvent) controls and activity was recorded for another 30 min. Stock (10x) solutions of glutamate, GABA, receptor (ant)agonists, endosulfan, and dl-amphetamine were diluted in cell culture medium to obtain the desired concentrations.

\subsection{Intracellular calcium imaging}

For further characterisation of iCell neurons (without added astrocytes), basal and stimulation-evoked changes in [Ca2+]i were measured at DIV4-8 using the Ca2+-sensitive ratio fluorescent dye Fura-2 AM as described previously (de Groot et al., 2014; De Groot et al., 2016). In short, cells were loaded with $5 \mu \mathrm{M}$ Fura-2 AM for $25 \mathrm{~min}$ at rt. Following $20 \mathrm{~min}$ to allow for de-esterification of the dye, Fura-2loaded cells were placed under the microscope under continuous superfusion with saline. Following a 5 min baseline measurement, cells were stimulated for $21 \mathrm{~s}$ with high-K+ saline (100 mM K+), $100 \mu \mathrm{M} \mathrm{GABA}, 100 \mu \mathrm{M}$ glutamate, or $100 \mu \mathrm{M} \mathrm{ACh}$ using an automated continuous superfusion system (AutoMate Scientific Inc., Berkeley, California). Hereafter, superfusion solution was switched back to saline allowing the cells to recover for $10 \mathrm{~min}$. Each dish of cells received only one stimulation to prevent potential effects of foregoing stimuli. 


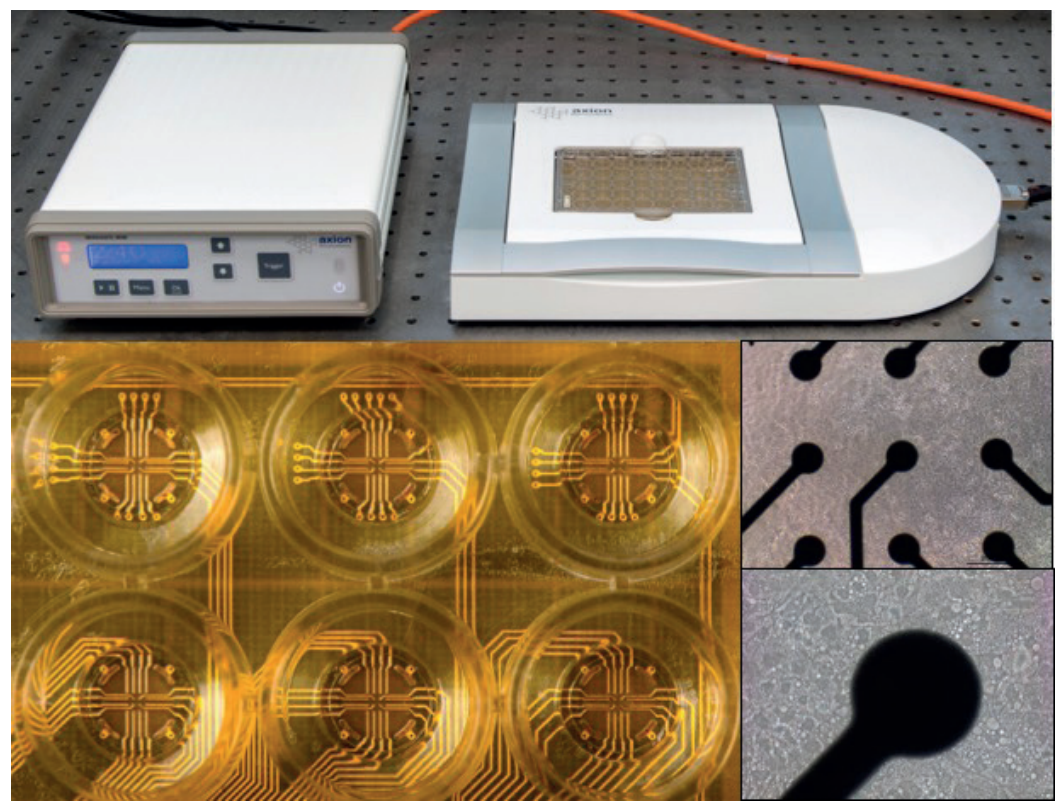

Figure 1. Experimental set up for measurements of spontaneous electrical network activity. Axion's Maestro (top) was used to recorded neuronal activity of human iPSC-derived neurons grown in 48-well MEA plates (bottom). Each well contains an electrode grid (bottom right, 10x magnification (top) and 20x magnification (bottom)) for non-invasive extracellular field recordings.

\subsection{Data analysis and statistics}

In order to calculate the percentage of astrocytes present in the different cultures the average number of astrocytes and total number of cells were counted (independently by two different researchers) from $\mathrm{n}$ images taken from $\mathrm{N}$ different cultures with Leica Application Suite Advanced Fluorescence software. Data are presented as mean \pm SEM.

To analyse (modulation of) spontaneous neuronal activity, raw data files were rerecorded to obtain Alpha Map files for further data analysis in NeuroExplorer. During the re-recording, spikes were detected using the AxIS spike detector (Adaptive threshold crossing, Ada BandFlt v2) with a variable threshold spike detector set at $6 \times$ (human iPSCs) or $7 \times$ (primary cortical culture) standard deviation (SD) of the internal noise level (rms) on each electrode. Next, spike count files were 
loaded into NeuroExplorer 5.007 software (Nex Technologies, Madison, Wisconsin) to determine the average mean spike rate (MSR; spikes/s) of all active electrodes (>0.01 spikes/s) per active well ( $\geq 1$ active electrode).

Custom-made excel macros were used to determine the development of spontaneous activity (MSR) over time and to determine the effect of test compounds on spontaneous activity. For the latter, a treatment ratio (TR) was established by: (MSRexposure/MSRbaseline) $\times 100 \%$. Next, TRs of exposures were normalized to the appropriate medium (glutamate, GABA, amphetamine) or DMSO (endosulfan) controls. Outliers in control and effect data (defined as not within average $\pm 2 \times \mathrm{SD}$ ) were removed $(<5 \%)$. All data are presented as mean \pm SEM from the number of electrodes (n) or wells $(\mathrm{N})$ indicated. A t-test was performed to determine significant changes in MSR in comparison to the control. Differences were considered significant if $\mathrm{p}<0.05$.

For calcium imaging experiments, changes in F340/F380 ratios reflecting changes in [Ca2+]i were analysed using custom-made MS-Excel macros. Recordings were normalized to baseline, and changes in [Ca2+]i are expressed as relative fluorescence units (RFU). Per stimulus condition, the percentage of active cells (defined as change in [Ca2+]i $>1.2$ RFU upon stimulation) was calculated and used for subsequent analysis of the amplitude of [Ca2+]i. Outliers (defined as cells with amplitude [Ca2+] i not within average $\pm 2 \times S D)$ were removed from the data set $(<5 \%)$. All data are presented as mean \pm SEM from the number of cells $(n)$ or dishes $(N)$ indicated.

\section{RESULTS}

\subsection{Immunofluorescent staining of human iPSC-derived neurons}

As an initial characterisation and to demonstrate their mixed neuronal nature, human iPSC-derived neurons were cultured for up to 1-2 weeks and $\beta$ (III)tubulin and glial fibrillary acidic protein (GFAP) antibodies were used to identify the presence of respectively neurons and astrocytes. HIP neurons (Figure 2, upper left) show a mixed phenotype with both neurons and astrocytes ( $21 \pm 7 \%$ astrocytes; $\mathrm{n}$ $=5$ images originating from 3 independent cultures). On the other hand, iCell neurons and DOPA.4U neurons have a more pure neuronal phenotype with very few astrocytes (not shown). Our initial experiments demonstrate that the heterogeneity of these cells can easily be increased by co-culturing the cells with pure astrocytes 
(Figure 2, lower left (DOPA.4U, $14 \pm 3 \%$ astrocytes; $\mathrm{n}=3$ images originating from a single culture) and upper right (iCell Neurons, $20 \pm 2 \%$ astrocytes; $n=4$ images originating from a single culture)). However, these co-cultures contain fewer astrocytes than the primary rat cortical culture ( $46 \pm 4 \% ; n=8$ images originating from 3 independent cultures).

To discriminate between different subtypes of neurons, we performed additional stainings for vesicular transporters of glutamate (vGluT) and GABA (vGAT). HIP neurons (not shown), iCell neurons (not shown) and iCell neurons co-cultured with pure astrocytes (Figure 2, lower right) all show clear expression of vGluT and vGAT, indicating the coexistence of respectively excitatory and inhibitory neuronal contacts.

These combined data indicate that our different human iPSC-derived (co-)cultures consist of excitatory glutamatergic neurons, inhibitory GABAergic neurons and (added) supporting astrocytes. The derived neuronal networks thus represent the cellular heterogeneity as observed in the in vivo brain and could be amenable to neurotoxicity testing.

\subsection{Spontaneous electrical activity of human iPSC-derived neurons}

To further test the applicability of these human iPSC-derived neurons for neurotoxicity testing, cells were cultured (without addition of astrocytes) on mwMEAs for up to 9 days to assess the development of spontaneous neuronal network activity over time. Electrical activity at DIV7-8 is depicted in spike raster plots, activity heat maps and individual spike traces (Figure 3). As can be seen in the raster plots and heat maps, neuronal activity differs between different cell models, with the iCell neurons and rat primary cortex cultures being the most active at DIV78. As also shown in table 1 , HIP neurons develop spontaneous neuronal activity following 4-6 days in culture, which slightly increases following 7-9 days in culture. Similarly, DOPA.4U neurons develop spontaneous neuronal activity that steadily increases over time. While the MSR of DOPA.4U neurons is clearly increased compared to HIP neurons, the \% active wells and \% active electrodes is comparable. 

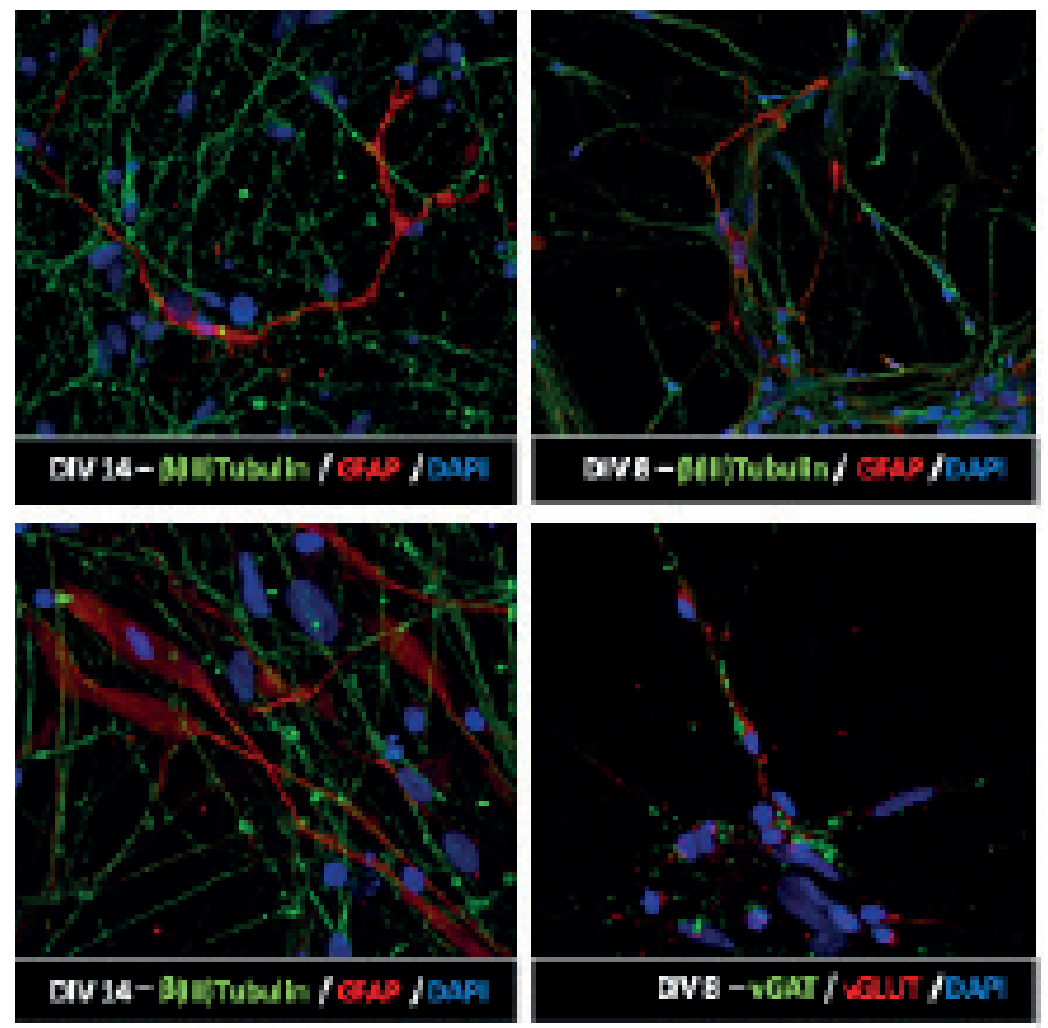

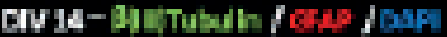

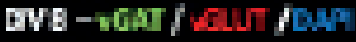

Figure 2. Immunofluorescent staining of human iPSC-derived neurons. Different human iPSCderived neurons were stained with $\beta$ (III)tubulin (green) and GFAP (red) antibodies to identify the presence of respectively neurons and astrocytes (HIP neurons, upper left; DOPA. $4 \mathrm{U}^{\circ}$ neurons, lower left; iCell neurons, upper right). Human iCell neurons were stained with vGAT (green) and vGluT (red) antibodies to demonstrate the presence of inhibitory (GABAergic) and excitatory (glutamatergic) neuronal contacts (lower right). Nuclei are stained with DAPI (blue). 


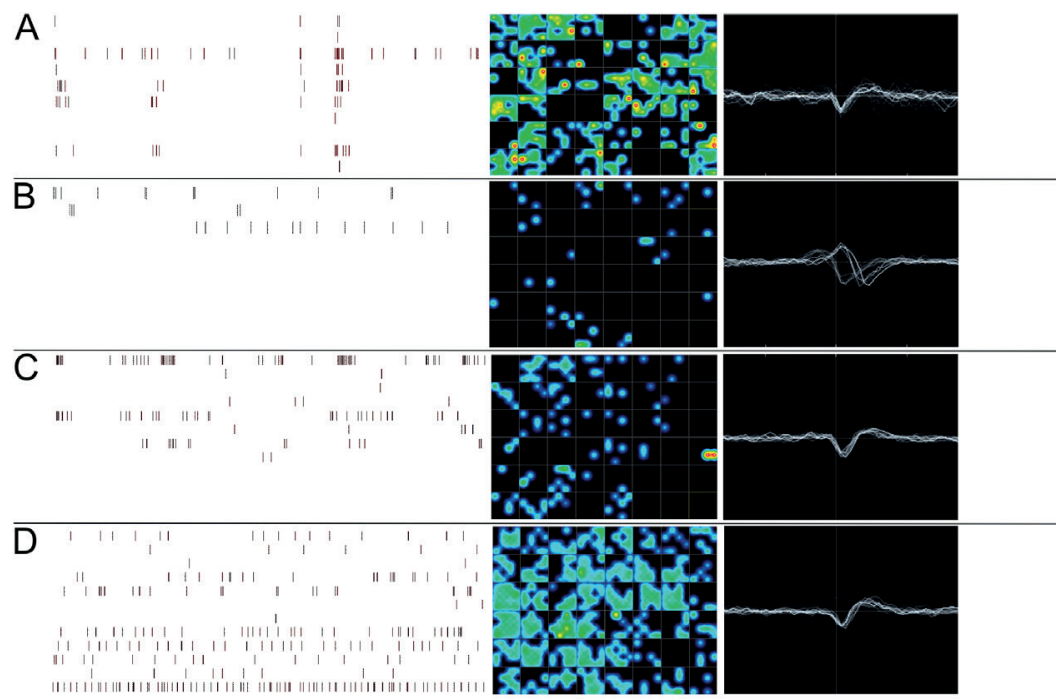

Figure 3. Spike raster plot (left; each row depicts one electrode in a representative example well, with every mark representing one spike in a 20 s interval), activity heat maps (middle; degree of activity in a 48-well plate depicted in false colour) and example traces of individual field potentials (right; recorded at a single representative electrode) illustrating the degree and pattern of neuronal activity of the primary rat cortical culture (A), HIP neurons (B), DOPA.4U neurons (C) and iCell neurons (D) at DIV7-8.

Table 1. Development of spontaneous neuronal activity in different human iPSC-derived neurons.

\begin{tabular}{|c|c|c|c|c|c|c|c|}
\hline & & DIV & & & DIV7- & & \\
\hline & MSR $^{1}$ & $\begin{array}{l}\text { \% active } \\
\text { wells }\end{array}$ & $\begin{array}{l}\text { \% active } \\
\text { electrodes }\end{array}$ & MSR $^{1}$ & $\begin{array}{l}\% \text { active } \\
\text { wells }\end{array}$ & $\begin{array}{l}\text { \% active } \\
\text { electrodes }\end{array}$ & $\mathbf{n} / \mathbf{N}^{2}$ \\
\hline HIP & 0.068 & 35.8 & 3.1 & 0.083 & 52.5 & 6.0 & $1917 / 120$ \\
\hline DOPA.4U & 0.214 & 40.0 & 5.9 & 0.240 & 48.4 & 7.6 & $1519 / 95$ \\
\hline iCell & 0.373 & 88.2 & 19.6 & 0.331 & 81.3 & 17.4 & $2301 / 144$ \\
\hline $\begin{array}{l}\text { Primary rat } \\
\text { cortical cells }\end{array}$ & n.a. ${ }^{3}$ & n.a. ${ }^{3}$ & n.a. ${ }^{3}$ & 0.353 & 94.8 & 32.0 & $768 / 48$ \\
\hline
\end{tabular}


Although MSR of HIP neurons and DOPA.4U neurons may further increase with prolonged differentiation in culture, such prolonged differentiation protocols make the cells less amenable to efficient and timely neurotoxicity screening. On the other hand, iCell neurons appear already maximally active following 4-6 days in culture, with a high MSR, \% active wells and \% active electrodes that slightly decreases with prolonged culture.

Primary rat cortical cultures, which are the current standard model for MEA recordings, are not yet active following 4-6 days in culture. Following 7-9 days of culture, activity of primary rat cortical cultures is comparable to the activity of iCell neurons at DIV4-6 (table 1), although it should be noted that the electrical activity of primary rat cortical cultures will increase with prolonged culture duration (see e.g. de Groot et al., 2016; Dingemans et al., unpublished).

\subsection{Modulation of electrical activity of iCell neurons}

While all tested cell cultures develop spontaneous activity, the rapid development of activity of iCell neurons can clearly be an advantage for neurotoxicity screening. Consequently, we used iCell neurons (without addition of astrocytes) for an initial brief functional screening using physiological, pharmacological and toxicological stimuli (Figure 4A-B). To verify the presence of glutamate and GABA receptors in iCell neurons, cells were exposed to different concentrations of glutamate $(0.3-30$ $\mu \mathrm{M})$ and GABA $(1-3 \mu \mathrm{M})$. Results demonstrate that spontaneous neuronal activity is concentration-dependently inhibited by high concentrations of glutamate and GABA following acute exposure (Figure 4A). Moreover, the neurotoxic insecticide endosulfan, which is a GABAA receptor antagonist, clearly increases neuronal activity (Figure 4B), comparable with primary rat cortical neurons challenged with endosulfan (Dingemans et al., unpublished) or the GABAA receptor antagonist lindane (Wallace et al., 2015). Finally, when iCell neurons were challenged with the widely used drug of abuse amphetamine, the MSR shows a concentrationdependent decrease (Figure 4B) as also observed for primary rat cortical neurons (Hondebrink et al., unpublished). 

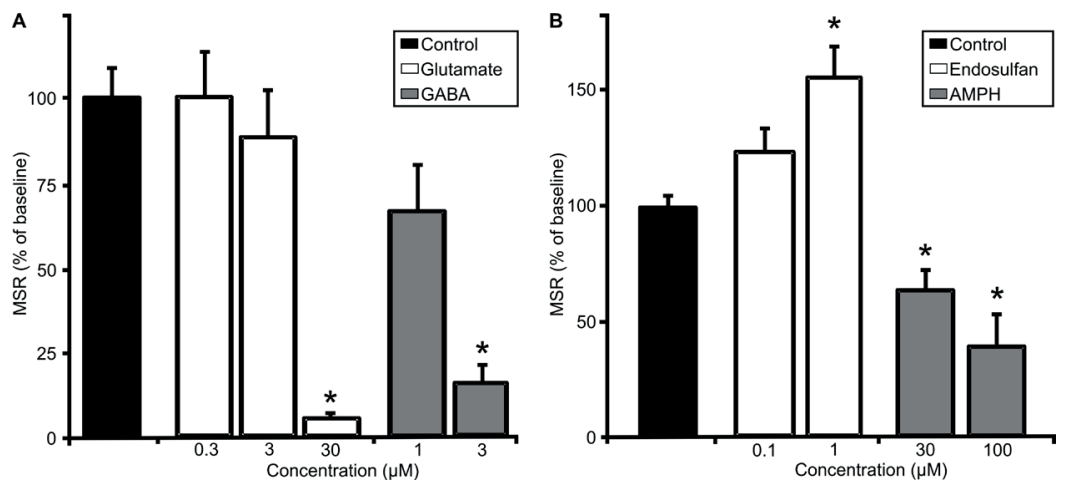

Figure 4. Physiological (A) and pharmacological (B) modulation of spontaneous network activity of iCell neurons. At DIV7-8, human iCell neurons were challenged with glutamate (0.3-30 $\mu \mathrm{M})$, GABA $(1-3 \mu \mathrm{M})$, endosulfan $(0.1$ or $1 \mu \mathrm{M})$ or amphetamine (AMPH; 30 or $100 \mu \mathrm{M}$ ). Data are expressed in MSR as \% of control; mean \pm SEM from 3-20 wells (N).
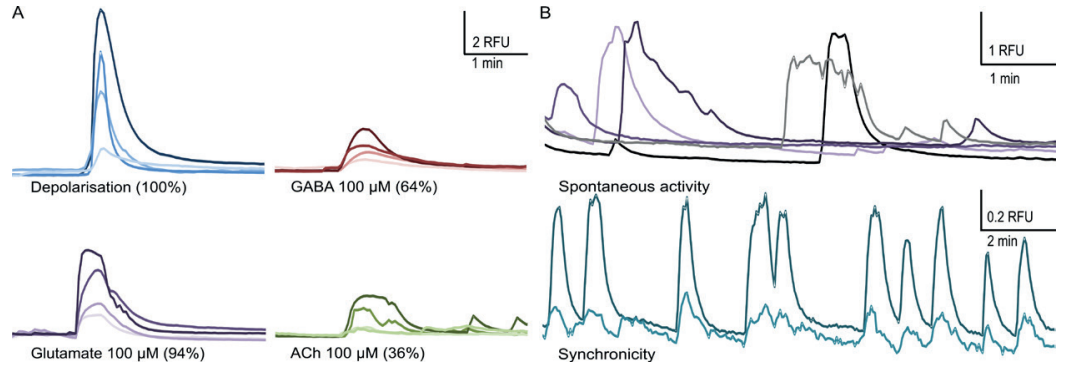

Figure 5. Representative traces demonstrating the increase in [Ca2+]i observed in individual iCell neurons following exposure to different stimuli as indicated (A). Percentages (in between brackets) indicate the fraction of cells responsive to the different stimuli (obtained from 21-57 individual cells in 3-4 independent experiments). Synchronicity and spontaneous activity were also observed (B).

\subsection{Functional characterisation of receptors and ion channels in iCell neurons} To further characterise iCell neurons with respect to the presence of receptors and ion channels, [Ca2+]i was measured using single-cell fluorescence microscopy. iCell neurons display a robust transient increase in [Ca2+]i upon depolarisation with $\mathrm{K}+$-containing saline (Figure $5 \mathrm{~A}$ ), demonstrating the presence of functional voltage-gated calcium channels. Also, stimulation with glutamate (100 $\mu \mathrm{M})$, GABA 
$(100 \mu \mathrm{M})$ and $\mathrm{ACh}(100 \mu \mathrm{M})$ results in an transient increase in [Ca2+]i, indicative for the presence of $\mathrm{Ca2}+$ permeable (ionotropic) glutamate receptors, GABA receptors and nicotinic ACh receptors (nACh-R), respectively (Figure 5A). In addition to stimulation-evoked transient increases in $[\mathrm{Ca2}+] \mathrm{i}$, iCell neurons exhibited spontaneous activity and occasionally synchronicity could be observed (Figure 5B).

\section{DISCUSSION}

Reliable detection of compound-induced neurotoxicity is challenging due to the high degree of integration of neuro-cellular processes in the (central) nervous system (CNS). Consequently, neurotoxicity testing currently relies strongly on animal studies, which are mandatory for new chemicals and CNS drugs, but require hundreds of rodents and up to a million euro per test compound. Successful and cost-effective replacement of these in vivo tests will thus drastically impact animal use for regulatory requirements. The use of integrated endpoints such as neuronal network activity allows for assessment of physiologically-relevant effects with considerable throughput and high sensitivity and specificity in vitro (McConnell et al., 2012; Nicolas et al., 2014; Valdivia et al., 2014), whereas direct effects on ion channels or neurotransmitter receptors could be assessed with targeted approaches such as single-cell calcium imaging. Importantly, the use of human cells prevents the need for interspecies extrapolation. While the costs of human iPSC-derived neurons are still high compared to primary cultures, the availability of human iPSC-derived neurons from particular brain areas and/or patients suffering from a particular neurological disorder opens the way to disease-specific neurotoxicity research (e.g., chemical-induced dopaminergic neurotoxicity or drugs screening using dopaminergic neurons like DOPA.4U).

Our immunocytochemical data (Figure 2) demonstrate that human iPSC-derived neurons from different suppliers efficiently capture the complexity and the high degree of integration of the brain in an in vitro system. iCell neurons, HIP neurons, DOPA.4U neurons and rat primary cortical cells all develop networks of inhibitory and excitatory neuronal cells when grown in vitro. The DOPA.4U and iCell neuron cultures had a relatively homogeneous neuronal nature with very little astrocytes present, whereas the HIP neuron culture had a more heterogeneous neuronal nature with a considerable fraction of astrocytes. The rat primary cortical cultures formed a heterogeneous network with presence of neurons and astrocytes, comparable with earlier findings ( $45 \%$ astrocytes; Görtz et al., 2004). 
While it is long known that astrocytes can affect the vulnerability of primary cultures to toxic insults (e.g. Dugan et al., 1995), adding astrocytes to pure neuronal cultures may also alter neuronal development and enhance cell signalling (Clarke and Barres, 2013; Odawara et al., 2014). Moreover, addition of astrocytes may affect chemical sensitivity as it has been shown using a mixed culture of NTERA-2 (NT2) cells that astrocytes are more sensitive to e.g. lead chloride and aluminium nitrate than neurons (Laurenza et al., 2013). We therefore also created co-cultures of astrocytes with DOPA.4U neurons or iCell neurons. It is in theory possible to design cell cultures with different ratios of neurons vs. astrocytes, however, it remains to be determined in future research if and how different ratios of astrocytes affect development of neuronal activity and sensitivity of human iPSC-derived neurons to toxic insults.

Using mwMEA recordings, human neuronal cultures were shown to be functional (i.e., spontaneously active) and amenable to neurotoxicity screening, with the iCell neurons (without addition of astrocytes) being most active at a short culture duration (DIV4-6; table 1, Figure 3). In accordance with previous studies (Robinette et al., 2011; Dingemans et al., unpublished), primary rat cortical cultures did not show any activity until DIV7. Around DIV7-9, primary cortical cultures and iCell neurons show a comparable degree of electrical activity. It should be noted though, that primary rat cortical cultures exhibit higher and stable levels of bursting and spiking following more prolonged culture (DIV10-14; Robinette et al., 2011; De Groot et al., 2016; Dingemans et al., unpublished) and that over time the degree of neuronal activity in DOPA.4U and HIP cultures may still develop to levels that equal or exceed those of iCell neurons at DIV4-6. While this should be taken into account in future studies, such studies may also need to focus on exploring the influence of different culture media on the speed and degree of neuronal development (see e.g. Bardy et al., 2015). Notably, our data on spontaneous network activity is derived from iCell and DOPA.4U neurons in the absence of (added) astrocytes. Future studies may therefore also need to focus on co-cultures of human iPSC-derived neurons with astrocytes, as it has been shown that this can enhance firing frequency and bursting levels (Odawara et al., 2014).

Next to MSR, a number of additional parameters could theoretically be extracted from the MEA recordings, including the percentage of active wells or percentage of active electrodes. However, in comparison to primary rat cortical neurons, the percentage of active wells is somewhat lower in human iPSCs, especially for HIP neurons and DOPA.4U neurons (table 1). This low percentage of active electrodes 
effectively hampers analysis of bursting parameters and synchrony of electrical network activity. While rat primary cultures develop synchronised bursting (already visible at DIV7-8, Figure 3), our data indicate that DOPA.4U neurons exert only little bursting behaviour (Figure 3 ) and bursting was virtually absent in iCell neurons and HIP neurons. Bursting parameters may help identify different groups of chemicals (Mack et al., 2014; Alloisio et al., 2015), although it has previously been shown that adding bursting parameters does not result in an increased sensitivity to detect neurotoxic chemicals in MEA-based screening approaches (Shafer et al., 2008; Defranchi et al., 2011; Dingemans et al., unpublished) and the MSR alone provides sufficient information for a first screening (McConnell et al., 2012). Additionally, the increasing throughput of mwMEA systems, currently up to 96-wells with only 8 electrodes per well, will continue to hamper detailed analysis of network synchrony. Nevertheless, future experiments using human iPSCs should focus on identification of culture conditions that allow bursting behaviour and increase MSR as well as the percentage of active wells and the percentage of active electrodes. In this respect it should be noted that co-culturing human iPSC-derived neurons with astrocytes may enhance synaptic maturation and transmission (Clarke and Barres, 2013) as well as firing frequency and bursting behaviour (Odawara et al., 2014).

While DOPA.4U and HIP neurons may become more active over time, the rapid development of electrical activity and the higher spike rate of iCell neurons is a clear advantage for fast and efficient neurotoxicological screening. We therefore used the iCell neurons for an explorative functional screening using different physiological, pharmacological and toxicological stimuli. This initial assessment demonstrated that MEA data obtained with iCell neurons are largely in line with previous data from primary cultures (Hogberg et al., 2011; McConnell et al., 2012; Dingemans et al., unpublished; Hondebrink et al., unpublished) and human embryonic stem cell-derived neurons (Ylä-Outinen, 2010). Moreover, iCell neurons challenged with endosulfan or amphetamine (Figure 4), show changes in neuronal activity comparable with primary rat cortical neurons (Dingemans et al., unpublished; Hondebrink et al., unpublished), indicating not only the presence and functionality of different neurotransmitter receptors, but also the potential for chemical neurotoxicity screening.

The presence of functional neurotransmitter receptors (GABA, glutamate and acetylcholine receptors) and voltage-gated calcium channels early in culture was confirmed using single cell calcium imaging (Figure 5). These findings extend on data from Dage et al. (2014), who showed that iCell neurons respond with robust calcium 
transients in response to GABA and NMDA exposure up till DIV28. Nevertheless, it is important to characterise any iPSC-derived model and elucidate the receptor profile at different days in culture prior to neurotoxicity testing as it has been shown that cellular properties can vary over time due to the maturation process of the cells (Dage et al., 2014; Odawara et al., 2014).

In conclusion, we show that human iPSC-derived neurons from different suppliers all form neuronal structures and develop spontaneous network activity. We also show that the cultures contain different neuronal subtypes, such as GABAergic and glutamatergic neurons. The human origin and short culture duration are major benefits of these commercial iPSCs, in particular for iCell neurons which are already spontaneously active following 4-6 DIV. However, the absence of bursting in these cells indicates that human iPSC-derived neuronal cultures may not yet be able to fully replace the rat primary cortical model. While addition of astrocytes and/or changes in culture conditions as well as additional characterisation, optimisation and toxicological validation are urgent pre-requisites, our data already indicate that human iPSC-derived neurons resemble primary cortical cultures when exposed to different physiological and pharmacological stimuli. Despite current limitations and disadvantages (high cost, absence of bursting, absence of astrocytes), human iPSC-derived neurons may enable animal-free neurotoxicity testing in the future and can already be used as a quick screening tool for chemical prioritisation and effect screening studies prior to animal studies or studies using primary cells.

\section{ACKNOWLEDGEMENTS}

Milou Dingemans, Aart de Groot, Gina van Kleef and other members of the Neurotoxicology Research Group as well as Jos Joore, Paul Vulto, Karlijn Wilschut, Remko van Vught and Nienke Wevers (Mimetas, Leiden, The Netherlands) are gratefully acknowledged for helpful discussions. We thank Rob Bleumink (IRAS, Utrecht University) for help with immunostainings. This work was funded by a grant from the National Centre for the Replacement, Refinement and Reduction of Animals in Research (NC3Rs; project number 50308-372160) and by the Faculty of Veterinary Medicine (Utrecht University, The Netherlands). 


\section{CONFLICT OF INTEREST STATEMENT}

The authors declare that they have no conflict of interests. 
Chapter 6

Human iPSC-Derived Neuronal Models for In Vitro Neurotoxicity Assessment

Anke M. Tukker, Fiona M.J. Wijnolts, Aart de Groot and Remco H.S. Westerink

Neurotoxicology Research Group, Toxicology and Pharmacology Division, Institute for Risk Assessment Sciences (IRAS), Faculty of Veterinary Medicine, Utrecht University, P.O. Box 80.177, NL-3508 TD Utrecht, The Netherlands.

NeuroToxicology (2018), volume 67, pp. $215-225$

DOI:10.1016/j.neuro.2018.06.007

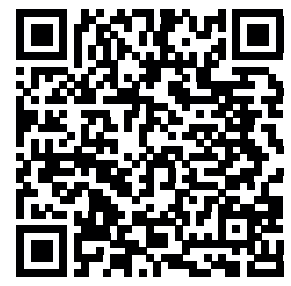




\section{ABSTRACT}

Neurotoxicity testing still relies on ethically debated, expensive and time consuming in vivo experiments, which are unsuitable for high-throughput toxicity screening. There is thus a clear need for a rapid in vitro screening strategy that is preferably based on human-derived neurons to circumvent interspecies translation. Recent availability of commercially obtainable human induced pluripotent stem cell (hiPSC)-derived neurons and astrocytes holds great promise in assisting the transition from the current standard of rat primary cortical cultures to an animalfree alternative.

We therefore composed several hiPSC-derived neuronal models with different ratios of excitatory and inhibitory neurons in the presence or absence of astrocytes. Using immunofluorescent stainings and multi-well micro-electrode array (mwMEA) recordings we demonstrate that these models form functional neuronal networks that become spontaneously active. The differences in development of spontaneous neuronal activity and bursting behaviour as well as spiking patterns between our models confirm the importance of the presence of astrocytes. Preliminary neurotoxicity assessment demonstrates that these cultures can be modulated with known seizurogenic compounds, such as picrotoxin (PTX) and endosulfan, and the neurotoxicant methylmercury (MeHg). However, the chemical-induced effects on different parameters for neuronal activity, such as mean spike rate (MSR) and mean burst rate (MBR), may depend on the ratio of inhibitory and excitatory neurons. Our results thus indicate that hiPSC-derived neuronal models must be carefully designed and characterized prior to large-scale use in neurotoxicity screening.

Keywords: in vitro neurotoxicity screening, human induced pluripotent stem cell-derived neuronal models, micro-electrode array (MEA), alternatives to animal testing, mixed neuronal cultures, astrocytes

\section{Highlights}

- $\quad$ hiPSC-derived models develop into networks with spontaneous activity and bursting

- $\quad$ hiPSC- derived models containing astrocytes exhibit synchronized activity

- Co-cultures can be modulated with seizurogenic compounds and neurotoxicants

- Inhibitory and excitatory neuron ratio may influence the chemical sensitivity

- $\quad$ hiPSC-derived models may already be used as prioritization tool in toxicity testing 


\section{INTRODUCTION}

Human induced pluripotent stem cell (hiPSC)-derived neuronal cultures are becoming increasingly important for in vitro neurotoxicity testing. These neuronal cultures could provide an alternative for costly, time consuming and ethically debated animal experiments or in vitro work with primary cultures. Moreover, there is a clear need for alternatives of human origin since animal-based models do not always mimic the human physiology and can therefore in some cases be poor predictors for human adverse outcomes (Hartung, 2008).

The use of hiPSC-derived neurons for neurotoxicity testing would circumvent the need for interspecies translation. As such, the recent commercial availability of these neuronal models holds great promise in assisting the transition from the current gold standard of rat primary cortical cultures (Hogberg et al., 2011; McConnell et al., 2012; Nicolas et al., 2014; Valdivia et al., 2014; Alloisio et al., 2015; Dingemans et al., 2016; Hondebrink et al., 2016; Vassallo et al., 2017) to hiPSC-derived models (Tukker et al., 2016). We have recently shown that these commercially available models have the potential to develop spontaneously active neuronal networks that can be used for screening and prioritization of chemically induced effects on neuronal activity (Tukker et al., 2016), whereas others have already shown that hiPSC-derived neurons exhibit the behaviour and function of mature neurons (Odawara et al., 2016; Hyysalo et al., 2017; Paavilainen et al., 2018). While costly in comparison to (rodent) primary cultures and (human) neural progenitor cells, an important benefit of hiPSC-derived neurons is the rapid development of a functional neuronal network, in contrast to the time consuming differentiation of hiPSCs into neural progenitor cells that subsequently need to be cultured into functional neurons, a process that can take several weeks (Görtz et al., 2004; Kuijlaars et al., 2016; Hyysalo et al., 2017) till months (Odawara et al., 2016; Paavilainen et al., 2018). Opting for these commercially obtainable cells comes with the additional benefit that they are available in high quantity, allowing for efficient screening (Anson et al., 2011). On the other hand, besides being costly, hiPSC-derived neurons have the disadvantage of being less well characterized for electrophysiological studies and have so far been little used for neurotoxicity screening.

In vitro screening models should represent the in vivo situation as closely as possible and must contain sufficient complexity to answer the research question (Westerink, 2013; Pamies and Hartung, 2017). With regard to neurotoxicity testing, this means that the model must capture the complexity and diversity of cell types of the human 
brain. It thus must form functional networks with a controlled balance of excitatory and inhibitory neurons as well as supportive cells. However, until now, most studies focused on cell cultures containing only hiPSC-derived neurons, whereas other neuronal cell types and/or astrocytes should also be included. Astrocytes, for example, play an important role in the regulation of the development of neurons (Tang et al., 2013) and also have been shown to enhance the development of neuronal networks and action potentials in human iPSC-derived co-cultures (Ishii et al., 2017). The addition of astrocytes to the neuronal model has also been reported to increase synchrony of the networks (Amiri et al., 2013). Moreover, inclusion of astrocytes also adds specific, physiologically relevant targets for toxic insults to the culture that would not be present in a pure neuronal model. Notably in this respect, there is growing evidence that astrocytes can play a protective role against chemicalinduced neurotoxicity (Takemoto et al., 2015; Wu, Yang, et al., 2017).

Currently, however, there is still a knowledge gap that has to be filled before cocultured hiPSC-derived models can replace the primary rat cortical cultures and become the new gold standard for mwMEA experiments. For example, the role of different cell types in hiPSC-derived neuronal cultures is largely unexplored and concerns regarding the immature phenotype, including limited bursting, have been raised (Meneghello et al., 2015). We therefore composed different (co-)cultures of iCell Glutaneurons ${ }^{\circledast}(\sim 90 \%$ excitatory glutamatergic neurons/10\% inhibitory GABAergic neurons) and iCell neurons ${ }^{\circledR}(\sim 30 \%$ excitatory glutamatergic neurons $/ 70 \%$ inhibitory GABAergic neurons) in the absence or presence of astrocytes to explore the role of different cell types in development of neuronal activity and suitability for in vitro neurotoxicity testing, including seizure liability testing.

\section{MATERIALS AND METHODS}

\subsection{Chemicals}

$\mathrm{N} 2$ supplement, penicillin-streptomycin (10.000 U/mL-10.000 $\mu \mathrm{g} / \mathrm{mL}$ ), Geltrex, phosphate-buffered saline (PBS), foetal bovine serum (FBS), DMEM, trypsin-EDTA, 4',6-diamidino-2-phenylindole (DAPI), donkey anti-rabbit Alexa Fluor ${ }^{\circledR} 488$ and donkey anti-mouse Alexa Fluor ${ }^{\circledR} 594$ were obtained from Life Technologies (Bleiswijk, The Netherlands). FluorSave was obtained from Calbiochem (San Diego, California, USA). iCell ${ }^{\circledR}$ Neurons Maintenance medium, iCell ${ }^{\circledR}$ Neurons Medium Supplement and Nervous System Supplement were 
obtained from Cellular Dynamics International (Madison, WI, USA). Rabbit anti- $\beta$ (III)-tubulin (Ab18207), mouse anti-S100 $\beta$ (Ab11178) and rabbit anti-vGluT1 (Ab104898) were obtained from Abcam (Cambridge, United Kingdom). Mouse anti-vGAT (131001) was obtained from Synaptic Systems (Göttingen, Germany). BrainPhys neuronal medium was obtained from StemCell Technologies (Cologne, Germany). Paraformaldehyde (PFA) was obtained from Electron Microscopy Sciences (Hatfield, Pennsylvania, USA). Picrotoxin (PTX), endosulfan ( $\alpha: \beta$ 2:1 99.9\%), methylmercury ( $\mathrm{MeHg}$ ), 50\% polyethyleneimine (PEI) solution, laminin, sodium borate, boric acid, bovine serum albumin and all other chemicals (unless described otherwise) were obtained from Sigma-Aldrich (Zwijndrecht, The Netherlands).

Stock solutions of endosulfan and MeHg were prepared in dimethyl sulfoxide (DMSO). For PTX, stock solutions were freshly prepared in ethanol (EtOH) directly before every experiment.

\subsection{Cell culture}

Cells were cultured at $37^{\circ} \mathrm{C}$ in a humidified $5 \% \mathrm{CO}_{2}$ incubator in line with supplier's instructions. All cell culture surfaces (coverslips and MEA plates) were pre-coated with $0.1 \%$ PEI solution diluted in borate buffer $(24 \mathrm{mM}$ sodium borate $/ 50 \mathrm{mM}$ boric acid in Milli-Q adjusted to $\mathrm{pH}$ 8.4) unless stated otherwise.

iCell ${ }^{\circledast}$ Astrocytes (Cellular Dynamics International, Madison, WI, USA) were thawed and cultured in DMEM with high glucose and 10\% FBS, $1 \%$ N2 supplement and 1\% penicillin-streptomycin, and were grown for 3-4 passages in Geltrex-coated culture flasks before using them in the co-cultures. Culture medium was refreshed every 3-4 days.

iCell ${ }^{\circledR}$ Glutaneurons and iCell ${ }^{\circledR}$ Neurons (Cellular Dynamics International, Madison, WI, USA) were thawed in Complete iCell Neurons Maintenance Medium supplemented with $2 \%$ iCell Neurons medium supplement, $1 \%$ penicillinstreptomycin and $1 \%$ laminin $(10 \mu \mathrm{g} / \mathrm{mL})$.

Before final plating, cells were pre-mixed into one of the four desired cultures: 1 ) monoculture of iCell ${ }^{\circledast}$ Glutaneurons, 2) co-culture of iCell ${ }^{\oplus}$ Glutaneurons and iCell ${ }^{\circledR}$ neurons, 3) co-culture of iCell ${ }^{\circledR}$ Glutaneurons and iCell ${ }^{\circledR}$ Astrocytes and 4) co-culture of iCell ${ }^{\circledR}$ Glutaneurons, iCell ${ }^{\circledR}$ neurons and iCell ${ }^{\circledR}$ Astrocytes. The different cell culture models and total number of cells/well or cells/chamber are described in table 1. 
Table 1. Composition and density of the different cell models.

\begin{tabular}{|c|c|c|}
\hline Type of culture & Cell types and ratio & $\begin{array}{l}\text { Seeding density/well or } \\
\text { chamber }\end{array}$ \\
\hline iCell ${ }^{\circledR}$ Glutaneuron monoculture & $100 \%$ Glutaneurons & 80,000 \\
\hline iCell ${ }^{\circledR}$ Glutaneuron-iCell ${ }^{\circledR}$ neuron co-culture & $\begin{array}{l}20 \% \text { iCell neurons } \\
80 \% \text { Glutaneurons }\end{array}$ & 80,000 \\
\hline $\begin{array}{l}\text { iCell }^{\circledR} \text { Glutaneuron-iCell }{ }^{\circledast} \text {-Astrocyte } \\
\text { co-culture }\end{array}$ & $\begin{array}{l}\text { 50\% Glutaneurons } \\
50 \% \text { Astrocytes }\end{array}$ & 150,000 \\
\hline $\begin{array}{l}\text { iCell }{ }^{\circledast} \text { Glutaneuron-iCell }{ }^{\circledast} \text { neuron- } \\
\text { iCell }{ }^{\circledast} \text { Astrocyte co-culture }\end{array}$ & $\begin{array}{l}40 \% \text { Glutaneurons } \\
10 \% \text { iCell neurons } \\
50 \% \text { Astrocytes }\end{array}$ & 140,000 \\
\hline
\end{tabular}

For immunofluorescent stainings, the different cell culture models were seeded on 8-chamber coverslips (Ibidi GmbH, Planegg, Germany) in $10 \mu \mathrm{L}$ droplets of cell suspension with a density of 80,000 cells/ droplet for the iCell ${ }^{\circledR}$ Glutaneuron monoculture and iCell ${ }^{\circledR}$ Glutaneuron-iCell ${ }^{\circledR}$ neuron co-culture; 150,000 cells/ droplet for the iCell ${ }^{\circledR}$ Glutaneuron-iCell ${ }^{\circledR}$ Astrocyte co-culture and 140,000 cells/ droplet for the iCell ${ }^{\circledR}$ Glutaneuron-iCell ${ }^{\circledR}$ neuron-iCell ${ }^{\circledR}$ Astrocyte co-culture. For multi-well micro-electrode array (mwMEA) experiments, cells were seeded as $10 \mu \mathrm{L}$ droplets of cell suspension, with the same seeding density/droplet as described above, directly over the electrode field of each well of a 48-well MEA plate (Axion Biosystems Inc., Atlanta, GA, USA). The droplets of cell suspension, both on the coverslips and on the MEA plates, were allowed to adhere for $1 \mathrm{~h}$ in a humidified incubator at $37^{\circ} \mathrm{C}$ and $5 \% \mathrm{CO}_{2}$. Next, room temperature (RT) supplemented BrainPhys medium (BrainPhys neuronal medium supplemented with $2 \%$ iCell Neuron supplement, $1 \%$ Nervous System supplement, $1 \%$ penicillin-streptomycin, $1 \% \mathrm{~N} 2$ supplement and $0.1 \%$ laminin $(10 \mu \mathrm{g} / \mathrm{mL}))$ was added ( $300 \mu \mathrm{L}$ for MEA, $200 \mu \mathrm{L}$ for chambers), and cells were placed back in the humidified incubator. After 1 day in vitro (DIV1), $50 \%$ of the medium was refreshed with RT supplemented BrainPhys medium. Hereafter, 50\% medium changes took place 3 times a week up till DIV21. 


\subsection{Immunocytochemistry}

The different hiPSC-derived neuronal (co-)cultures were fixated as described previously (Tukker et al., 2016) on DIV7, 14 and 21 with $4 \%$ PFA in $0.1 \mathrm{M} \mathrm{PBS} \mathrm{(pH}$ 7.4) for $15 \mathrm{~min}$ at RT. Briefly, following fixation coverslips were quenched for PFA, permeabilized and incubated for $20 \mathrm{~min}$ at RT with $20 \mathrm{mM} \mathrm{NH}_{4} \mathrm{Cl}$ in blocking buffer ( $2 \%$ bovine serum albumin and $0.1 \%$ saponin in PBS). Next, coverslips were incubated overnight at $4^{\circ} \mathrm{C}$ with rabbit anti- $\beta$ III tubulin (final dilution 1:250), mouse anti-S100 $\beta$ (final dilution 1:500), mouse anti-vGAT (final dilution 1:1000) and/or rabbit anti-vGluT1 (final dilution 1:1500) in blocking buffer. Hereafter, coverslips were washed 3 times with blocking buffer and incubated with donkey anti-mouse Alexa Fluor ${ }^{\circledR} 488$ and/or donkey anti-rabbit Alexa Fluor ${ }^{\circledR} 594$ (final dilution 1:100) for $30 \mathrm{~min}$ at RT in the dark. Coverslips were incubated for 2-3 min with $200 \mathrm{nM}$ DAPI at RT in the dark for nuclear staining. Coverslips were again washed 3 times with blocking buffer and sealed with FluorSave. The coverslips were stored at $4^{\circ} \mathrm{C}$ in the dark until further use. Immunostained coverslips were visualised with a Leica SPEII Confocal microscope (Leica DMI4000 equipped with TCS-SPE-II) using a $20 x$ oil immersion objective (N.A. 1.4-0.7). Images were captured as .tiff files using Leica Application Suite Advanced Fluorescence software (LAS AF version 2.6.0; Leica Microsystems GmbH, Wetzlar, Germany).

\subsection{MEA measurements}

The different hiPSC-derived neuronal (co-)cultures were cultured on 48-well PEIcoated MEA plates (Axion Biosystems Inc., Atlanta, GA, USA). Each well contains an electrode array of 16 nanotextured gold micro-electrodes (40-50 $\mu$ m diameter; 350 $\mu \mathrm{m}$ centre-to-centre spacing) with 4 integrated ground electrodes, yielding a total of 768 channels, which can be recorded at the same time. Spontaneous network activity was recorded at $37^{\circ} \mathrm{C}$ without further climate control at DIV4, 7, 9, 11, 14, 16, 18 and 21 as described previously (de Groot et al., 2014; Dingemans et al., 2016). Briefly, signals were recorded using a Maestro 768-channel amplifier with integrated heating system and temperature controller and a data acquisition interface (Axion Biosystems Inc., Atlanta, GA, USA). Data acquisition was managed with Axion's Integrated Studio (AxIS version 2.4.2.13). MEA plates were allowed to equilibrate for $\sim 5$ min prior to the 30 min recording of spontaneous activity. Raw data files were obtained by sampling the channels simultaneously with a gain of 1200x and a sampling frequency of $12.5 \mathrm{kHz} /$ channel using a band-pass filter $(200-5000 \mathrm{~Hz})$. 
To determine effects of test compounds on spontaneous activity (spiking and bursting behaviour) of the different hiPSC (co-)cultures, activity was recorded at DIV21. Immediately after this baseline recording, wells were exposed (dilution 1:10) to test compounds or the appropriate solvent control (DMSO or EtOH) and activity was recorded for another $30 \mathrm{~min}$. Stock solutions of endosulfan, $\mathrm{MeHg}$ and PTX were diluted in supplemented BrainPhys culture medium to obtain the desired concentrations. In all experiments the solvent concentration never exceeded $0.1 \% \mathrm{v} / \mathrm{v}$.

\subsection{Data analysis and statistics}

After recording spontaneous activity, raw files were re-recorded to obtain Alpha Map files for further data analysis. During the re-recording, spikes were detected using the AxIS spike detector (Adaptive threshold crossing, Ada BandFIt v2) with a variable threshold spike detector set at $7 \mathrm{x}$ standard deviation (SD) of internal noise level (rms) on each electrode. The resulting spike count files were loaded into NeuroExplorer 5.020 software (Nex Technologies, Madison, Wisconsin) for further analysis. For all experiments, spike files were loaded in Neural Metric (version 2.04, Axion Biosystems). Only active electrodes (MSR $\geq 6 \mathrm{spikes} / \mathrm{min}$ ) in active wells ( $\geq 1$ active electrode) were included in the analysis. Mean burst rate (MBR) was extracted with the Poisson Surprise method with a minimal surprise of 10 (Legéndy and Salcman, 1985) and a minimum bursting frequency of 0.001 bursts/sec. Network bursts were extracted using the adaptive threshold method.

For constructing developmental curves, the full 30 min recordings obtained at DIV4, 7, 9, 11, 14, 16, 18 and 21 were used for data analysis. Neural metric outputs were loaded in a custom-made Excel macro to select only those electrodes that fit the activity criteria. Only wells with a minimum of 2 bursting electrodes were used to calculate network and synchronicity parameters. Percentages of spiking wells were calculated compared to the number of plated wells, percentages of bursting wells were calculated compared to the number of spiking wells, and percentages of network bursting wells were calculated compared to the number of bursting wells.

The effects of test compounds were determined in exposure experiments at DIV21 in which the baseline activity prior to exposure was compared to activity following exposure. To prevent inclusion of artifacts, a correction for the time it took to expose the wells was performed. For example, if it took $3 \mathrm{~min}$ to expose the mwMEA plate, the first 3 min of the recording were not used for data analysis, only the subsequent $30 \mathrm{~min}$. 
For exposure experiments, a custom-made excel macro was used to establish treatment ratios per well for the different metric parameters (table 2) by expressing the parameter ${ }_{\text {exposure }}$ as a percentage change compared to the parameter $_{\text {baseline }}$. Only those electrodes in a well that fit the activity criteria during baseline recording were used to calculate well averages. All electrodes defined as active in baseline recordings were then used to calculate well averages during exposure. Next, treatment ratios were normalized to the appropriate vehicle control. Outliers in control and effect data (defined as not within average \pm 2 $x$ SD) were excluded from further data analysis (2\%). All data are presented as mean \pm SEM from the number of wells ( $n$, with a minimum of 2 wells). SEM is used as statistical measure as it reflects the accuracy of the reported value with respect to the "true mean" of the population assessed, rather than providing a descriptive measure that reflects the variability within the population such as the SD (Pleil, 2016), and for easy comparability with published datasets (e.g. Alloisio et al., 2015; Brown et al., 2016; Dingemans et al., 2016; Hogberg et al., 2011; Strickland et al., 2016). A t-test was performed to determine significant changes $(p<0.05)$ in MSR or MBR compared to the vehicle control. 
Table 2. Overview and description of used metric parameters.

\section{Metric parameter Description}

Mean spike rate (MSR) Total number of spikes divided by recording time ( $\mathrm{Hz})$

ISI coefficient of

variation

Burst duration

Number of spikes per

burst

Mean ISI within burst

Median ISI within

burst

Inter-burst interval

Burst frequency

IBI coefficient of

variation

Burst percentage

Network burst

frequency

Network burst duration Average time from the first spike till the last spike in a network burst (s)

Number of spikes per

network burst

Mean ISI within

network burst

Median ISI within

network burst

Number of spikes per

network burst/channel

Network burst

percentage

Network IBI coefficient Standard deviation of network IBI divided by the mean network IBI. Measure

of variation

Network normalized

duration IQR

Standard deviation ISI (time between spikes) divided by the mean ISI. Measure for spike regularity: 0 indicates perfect spike distribution, >1 signals bursting Average time from the first spike in a burst till the last spike (s)

Average number of spikes occurring in a burst

Mean inter-spike interval within a burst (s)

Median inter-spike interval within a burst (s)

Time between the last spike of a burst and the first spike of a subsequent burst

(s)

Total number of bursts divided by recording time $(\mathrm{Hz})$

Standard deviation of IBI divided by the mean IBI. Measure for burst regularity

Percentage of total number of spikes occurring in a burst

Total number of network bursts divided by recording time $(\mathrm{Hz})$

Average number of spikes occurring in a network burst

Average of the mean ISIs within a network burst (s)

Average of the median ISIs within a network burst (s)

Average number of spikes in a network burst divided by the electrodes

participating in that burst

Percentage of total spikes occurring in a network burst

of network burst rhythmicity: value is small when bursts occur at regular interval and increases when bursts occur more sporadic

Interquartile range of network bursts durations. Measure for network burst duration regularity: larger values indicate wide variation in duration. 
Area under normalized Area under inter-electrode cross-correlation normalized to the auto-

cross-correlation correlations. The higher the value, the greater the synchronicity of the network.

Full width at half Width at half height of the normalized cross-correlogram. Measure for

height of normalized network synchrony: the higher the value, the less synchronised the network is.

cross-correlation

\section{RESULTS}

\subsection{Immunofluorescent stainings of the different human iPSC-derived neuronal models}

As a first characterization, the iCell ${ }^{\circledR}$ Glutaneuron monoculture was cultured for up to 3 weeks and labelled with $\beta$ (III)tubulin antibodies at DIV21 to identify the presence of neurons and to visualise network formation (Fig. IA). To discriminate between subtypes of neurons in the iCell ${ }^{\circledR}$ Glutaneuron-iCell ${ }^{\circledR}$ neuron co-culture, vesicular transporters of glutamate (vGlut) and GABA (vGAT) were stained (Fig. 1B). This co-culture shows a clear expression of vGlut and vGAT indicating the coexistence of respectively excitatory and inhibitory neurons. Both cultures show a pure neuronal phenotype. The heterogeneity of the culture can be increased by co-culturing the neurons with astrocytes.

Co-cultures containing astrocytes were also cultured up to 3 weeks and were labelled with S10o $\beta$ and $\beta$ (III)tubulin antibodies at DIV7, 14 and 21 to identify the presence of respectively astrocytes and neurons (Fig. 2A-B). Both models show network formation with a high degree of complexity. Network density and complexity increases with prolonged culture duration. In both models, neurons and astrocytes distribute evenly to form mixed networks. Visual observation indicates that the ratio astrocyte to neuron is stable during the entire culture duration.

These results show that the different hiPSC-derived (co-)cultures all consist of inhibitory GABAergic and excitatory glutamatergic neurons and, when added, supporting astrocytes. The data also indicate that neuronal networks are formed, suggesting that they could be suitable for neurotoxicity screening. 

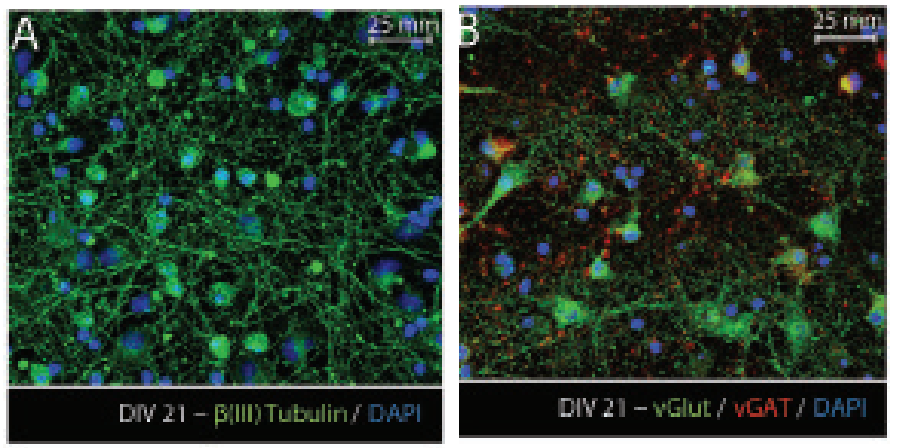

Figure 1. Immunofluorescent stainings of the iCell ${ }^{\circledR}$ Glutaneuron monoculture (A) and iCell ${ }^{\circledast}$ Glutaneuron-iCell ${ }^{\circledast}$ neuron co-culture (B). At DIV21 the monoculture was stained with $\beta($ III) tubulin (green) to identify neurons and show network formation (A). To demonstrate the presence of GABAergic and glutamatergic neurons in the iCell ${ }^{\circledR}$ Glutaneuron-iCell ${ }^{\circledR}$ neuron co-culture cells were stained with vGAT (red) and vGlut (green) (B). Nuclei are stained with DAPI (blue). Scale bar depicts $25 \mu \mathrm{m}$
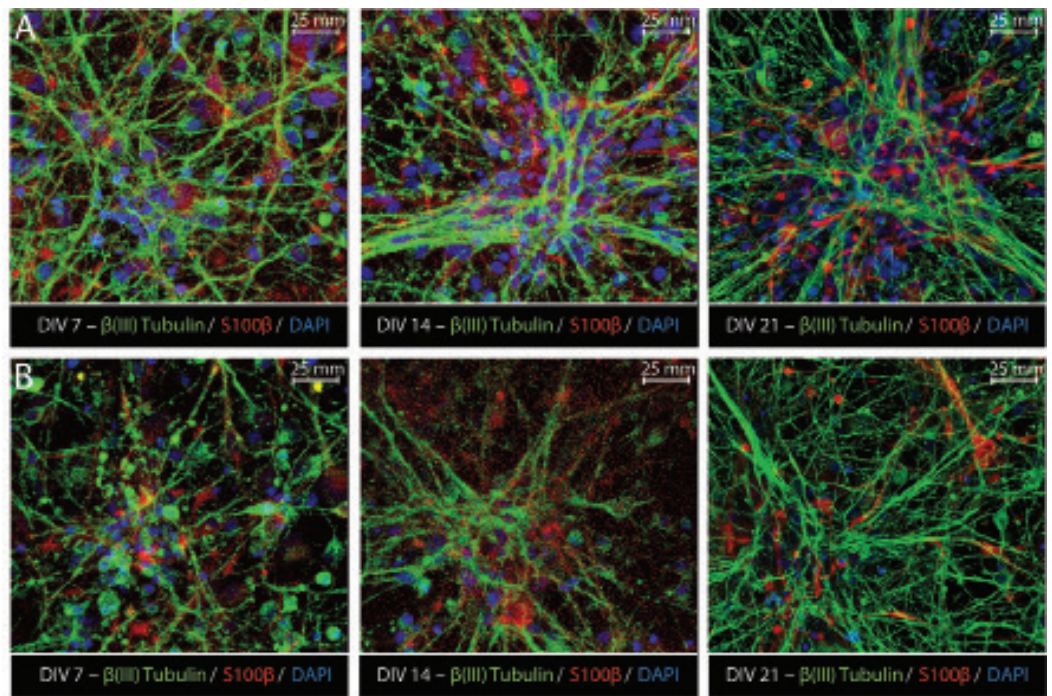

Figure 2. Immunofluorescent stainings of the different hiPSC-derived neuronal co-cultures with astrocytes. At DIV7 (left), 14 (middle) and 21 (right) cultures were stained with $\beta(\mathrm{III})$ tubulin (green) and S100 $\beta$ (red) to identify respectively neurons and astrocytes in the iCell ${ }^{\circledR}$ Glutaneuron-Astrocyte co-culture (A) and iCell ${ }^{\circledast}$ Glutaneuron-iCell ${ }^{\circledast}$ neuron-Astrocyte co-culture (B). Nuclei are stained with DAPI (blue). Scale bar depicts $25 \mu \mathrm{m}$. 


\subsection{Development of spontaneous electrical activity and bursting}

In order to further test the applicability of our different hiPSC-derived neuronal models for neurotoxicity screening, cells were cultured for up to 21 days on mwMEAs. Development of spontaneous neuronal network activity and bursting behaviour over time were assessed. The spontaneous electrical activity at DIV7, 14 and 21 is depicted in spike raster plots (Fig. 3). The plots show that all models develop spontaneous neuronal activity, although the pattern of activity differs strongly between the models. Models without astrocytes (Fig. 3A-B) do not show synchronicity, whereas models with astrocytes synchronize their activity with prolonged culture duration (Fig. 3C-D). All tested models develop bursting, but the amount of bursts differs per culture. The iCell ${ }^{\circledR}$ Glutaneuron monoculture and iCell ${ }^{\circledR}$ Glutaneuron-iCell ${ }^{\circledR}$ neuron co-culture show few bursting electrodes (Fig. 3A-B), in contrast to the co-cultures containing astrocytes where almost all active electrodes show bursting (Fig. 3C-D).

In all tested cultures the mean spike rate (MSR) is relatively stable over time (Fig. $4 \mathrm{~A}-5 \mathrm{~A})$. Addition of the more inhibitory iCell ${ }^{\circledR}$ neurons to iCell ${ }^{\circledR}$ Glutaneurons has limited effect on MSR or the percentage of active wells (Fig. 4A). Similarly, addition of astrocytes to iCell ${ }^{\circledR}$ Glutaneurons or to a co-culture of iCell ${ }^{\circledR}$ Glutaneurons and iCell ${ }^{\circledast}$ neurons (Fig. 5A) has limited effects on spike rates. However, addition of astrocytes does increase the percentage of active wells 

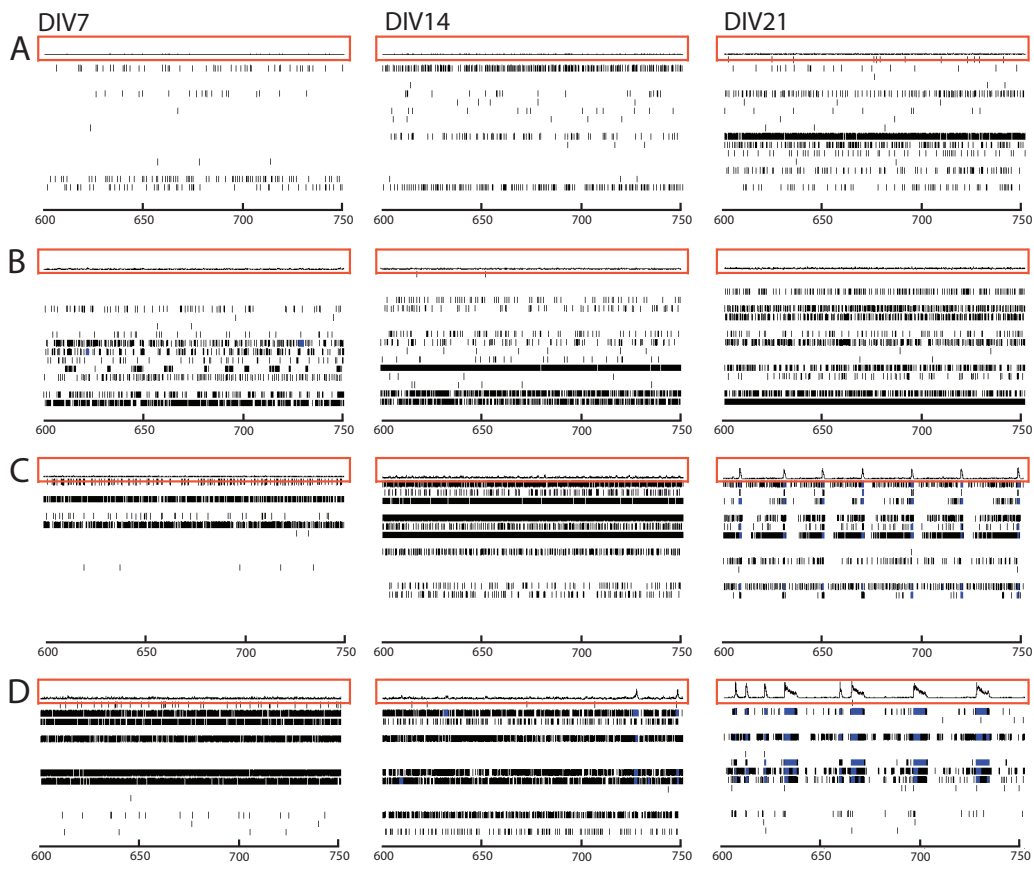

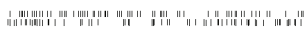
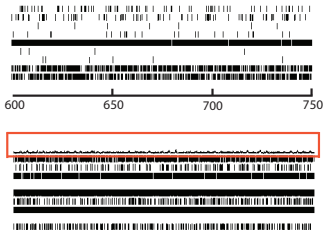

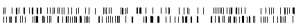
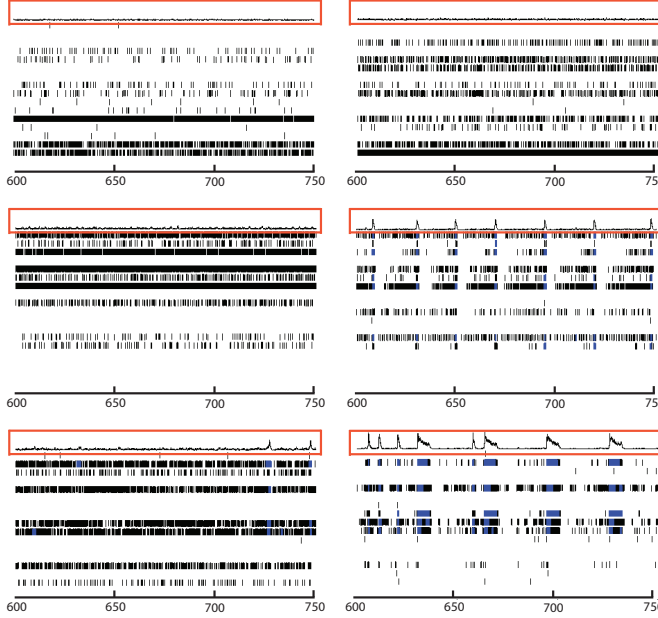

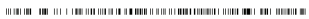
(1)

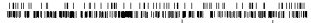

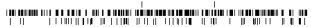

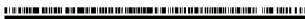

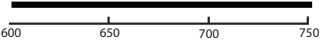

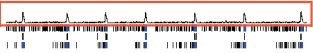

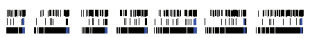

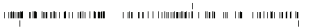

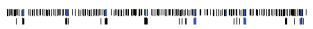

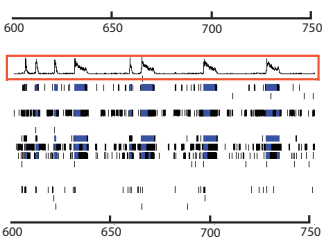

Figure 3. Spike raster plots of different hiPSC-derived cultures. Each row depicts one electrode in a representative example well, with each tick representing one spike (field potential) in a $150 \mathrm{~s}$ interval. This illustrates the degree and pattern of activity of the iCell ${ }^{\circledR}$ Glutaneuron monoculture (A), the iCell ${ }^{\circledast}$ Glutaneuron-iCell ${ }^{\circledast}$ neuron co-culture (B), the iCell ${ }^{\circledR}$ Glutaneuron-Astrocyte coculture (C) and the iCell ${ }^{\circledR}$ Glutaneuron-iCell ${ }^{\circledR}$ neuron-Astrocyte co-culture (D) at DIV7 (left), DIV14 (middle) and DIV21 (right). The cumulative trace in the orange box above each raster plot depicts the population spike time histogram indicating the synchronized activity between the different electrodes (network burst). 

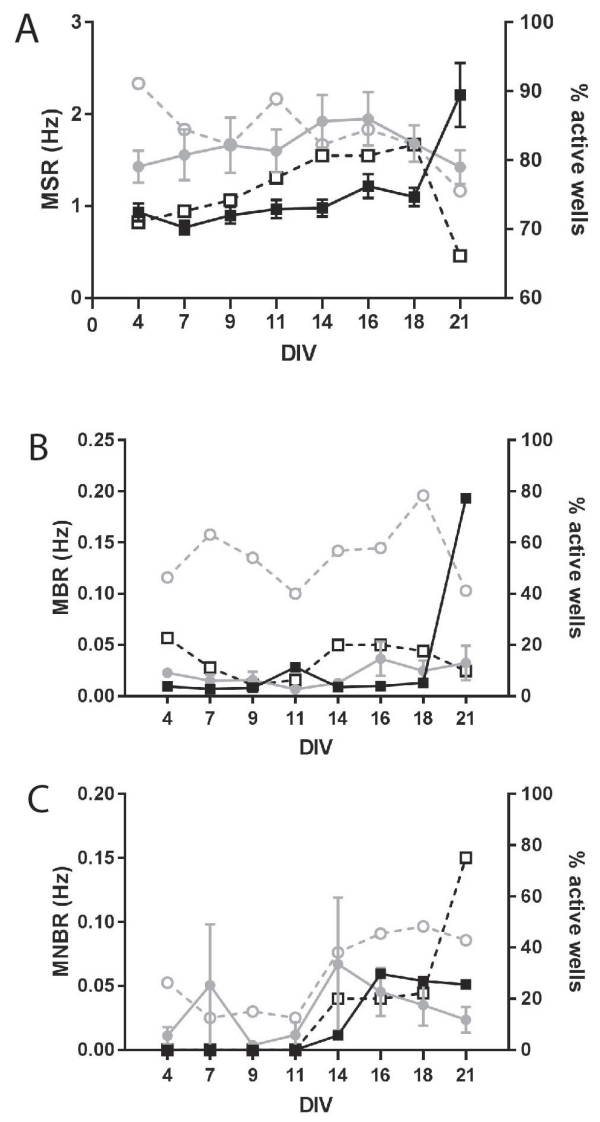
iCell@ Glutaneuron
iCell $($ Glutaneuron - iCell( neuron
- . iCell( Glutaneuron $\%$ active wells
iCell $($ Glutaneuron - iCell neuron $\%$ active wells

Figure 4. Development of spontaneous neuronal activity and (network) bursting in different h-iPSC-derived neuronal (co-)cultures. A) Mean spike rate (MSR ( $\mathrm{Hz}$ ); solid lines) and percentage of spiking wells (dashed lines) are depicted in for the iCell ${ }^{\circledR}$ Glutaneuron monoculture (black lines; $\mathrm{n}=41-51, \mathrm{~N}=3$ ) and iCell $^{\circledR}$ Glutaneuron-iCell ${ }^{\circledR}$ neuron co-culture (grey lines; $\mathrm{n}=34-41, \mathrm{~N}$ = 4). B) Mean burst rate (MBR (Hz); solid lines) and percentage of bursting wells (dashed lines) are depicted for the iCell ${ }^{\circledR}$ Glutaneuron monoculture (black lines; $\mathrm{n}=2-10, \mathrm{~N}=3$ ) and iCell $^{\oplus}$ Glutaneuron-iCell ${ }^{\circledR}$ neuron co-culture (grey lines; $n=14-29, N=4$ ). C) Mean network burst rate (MNBR (Hz); solid lines) and percentage of network bursting wells (dashed lines) are depicted for the iCell ${ }^{\oplus}$ Glutaneuron monoculture (black lines; $\mathrm{n}=2-3, \mathrm{~N}=3$ ) and iCell ${ }^{\circledR}$ Glutaneuron-iCell ${ }^{\oplus}$ neuron co-culture (grey lines; $n=2-14, N=4$ ). Data are expressed as mean \pm SEM from $n$ wells and $\mathrm{N}$ cultures. 
In the absence of astrocytes, the mean burst rate (MBR) is limited and relatively stable over time (Fig. 4B). Addition of astrocytes increases both the MBR and the percentage of bursting wells in a time-dependent manner (Fig $5 \mathrm{~B}$ ). While the iCell ${ }^{\circledR}$ Glutaneuron-Astrocyte co-culture shows a higher percentage of bursting wells than the iCell ${ }^{\circledast}$ Glutaneuron-iCell ${ }^{\circledR}$ neuron-Astrocyte co-culture, both models show an optimal window for MBR and percentage bursting wells around DIV16-21.

The onset of network bursts also shows some time-dependence, with an increase of the mean network burst rate (MNBR) after 1-2 weeks in culture (Fig. $4 \mathrm{C} / 5 \mathrm{C}$ ). In the absence of astrocytes, both the MNBR and the percentage of wells with network bursts are limited. Addition of astrocytes to the iCell ${ }^{\circledR}$ Glutaneuron and iCell ${ }^{\circledR}$ Glutaneuron-iCell ${ }^{\circledast}$ neuron co-culture has only a limited effect on MNBR, but clearly increases the percentage of wells with network bursts later in culture, with an optimal window for MBR and percentage bursting wells around DIV16-21 (Fig. 5C).

Further analysis of different parameters for neuronal activity highlights additional differences between our models. Addition of astrocytes decreases burst duration, but increases network burst duration (Fig. 6A). The inter-burst interval (IBI) decreases in the cultures containing astrocytes (Fig. 6B solid line), whereas the IBI coefficient of variation is comparable at the end of culture time for all models (Fig. 6B dashed line). At the peak of the activity of all models (DIV16-18), the number of spikes in a burst is higher in the cultures without astrocytes (Fig. $6 \mathrm{C}$ solid line). However, at this time the percentage of spikes that occur in a burst is higher in the astrocyte containing cultures (Fig. $6 \mathrm{C}$ dashed line). In regard to the number of spikes per network burst and the percentage of spikes in a network burst, the models behave comparable except for the onset of network bursting (Fig. 6D). Astrocyte containing co-cultures show higher synchrony as indicated by the area under normalized cross correlation (Fig. 6E solid line), which is confirmed by the lower full width at half height (FWHH, Fig. 6E dashed line). 

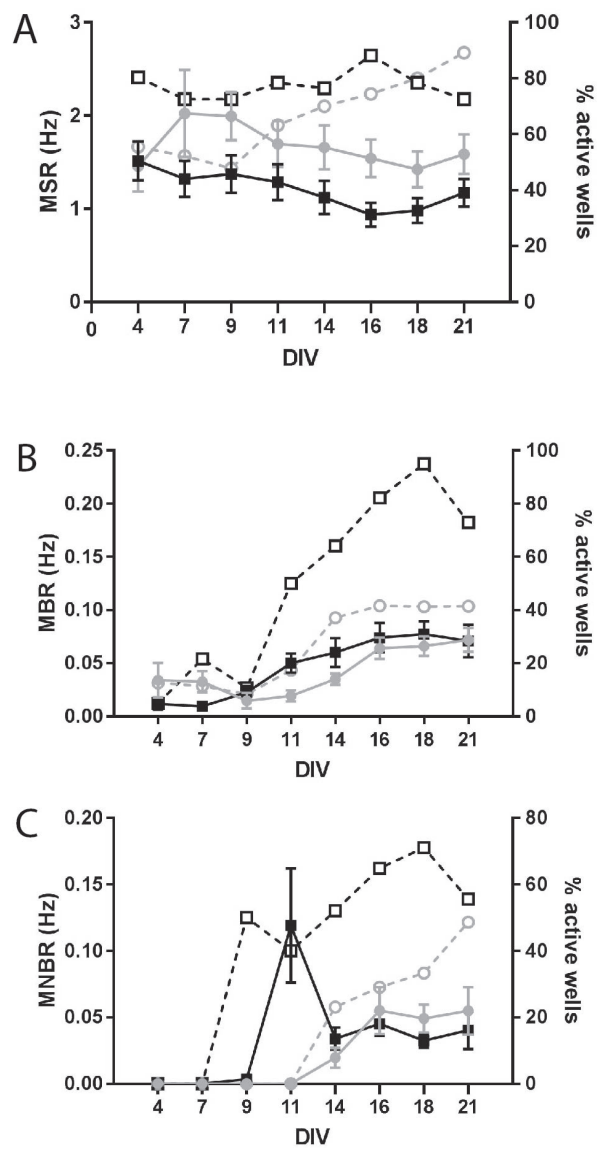

- iCell $\left({ }_{B}\right.$ Glutaneuron Astrocyte MSR iCell@ Glutaneuron - iCell® neuron - Astrocyte MSR

-n. iCellß Glutaneuron Astrocyte \% active wells

iCell( Glutaneuron - iCell(B) -o. neuron - Astrocyte \% active wells

Figure 5. Development of spontaneous neuronal activity and (network) bursting in different hiPSC-derived neuronal co-cultures with astrocytes. A) Mean spike rate (MSR (Hz); solid lines) and percentage of spiking wells (dashed lines) are depicted for the iCell ${ }^{\circledast}$ Glutaneuron-Astrocyte co-culture (black lines; $\mathrm{n}=43-72, \mathrm{~N}=4$ ) and iCell ${ }^{\circledR}$ Glutaneuron-iCell ${ }^{\circledR}$ neuron-Astrocyte coculture (grey lines; $n=34-41, N=4)$. B) Mean burst rate (MBR (Hz); solid lines) and percentage of bursting wells (dashed lines) are depicted for the iCell ${ }^{\circledR}$ Glutaneuron-Astrocyte co-culture (black lines; $\mathrm{n}=2$-38, $\mathrm{N}=4$ ) and iCell $^{\oplus}$ Glutaneuron-iCell ${ }^{\circledR}$ neuron-Astrocyte co-culture (grey lines; $\mathrm{n}$ $=4-37, N=5)$. C) Mean network burst rate (MNBR (Hz); solid lines) and percentage of network bursting wells (dashed lines) are depicted for the iCell ${ }^{\circledR}$ Glutaneuron-Astrocyte co-culture (black lines; $n=2-27, N=4$ ) and iCell ${ }^{\circledast}$ Glutaneuron-iCell ${ }^{\circledast}$ neuron-Astrocyte co-culture (grey lines; $n=$ 6-18, $N=5$ ). Data are expressed as mean \pm SEM from $n$ wells and $N$ cultures. 

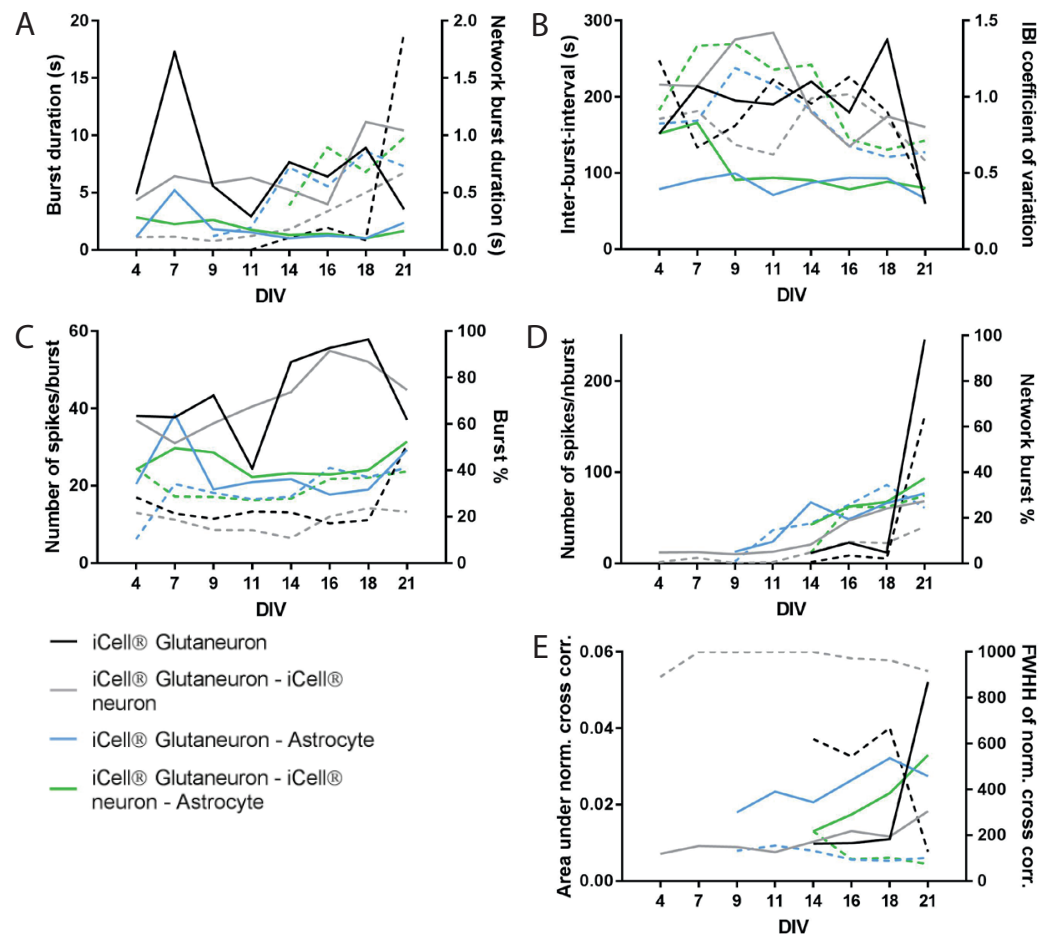

Figure 6. Development of spike and (network) burst activity and synchronicity of the different hiPSC-derived neuronal models. A) Burst duration (s) and network burst duration (s). B) Interburst interval (IBI; (s)) and IBI coefficient of variation. C) Number of spikes/burst and percentage of spikes in burst. D) Number of spikes/network burst and percentage of spikes in network burst. E) Area under normalized cross correlation and full width at half height (FWHH). Solid lines match the left $y$-axis, whereas dashed lines match the right $y$-axis. Data of the iCell ${ }^{\circledR}$ Glutaneuron monoculture are depicted in black, of the iCell ${ }^{\circledR}$ Glutaneuron-iCell ${ }^{\circledR}$ neuron co-culture in grey, of the iCell ${ }^{\circledR}$ Glutaneuron-Astrocyte co-culture in blue, and of the iCell ${ }^{\oplus}$ Glutaneuron-iCell ${ }^{\circledR}$ neuronAstrocyte co-culture in green. Data are expressed as mean; for SEM, $\mathrm{n}$ and $\mathrm{N}$ see table $\mathrm{S} 1$ in the supplemental data. 


\subsection{Preliminary neurotoxicity assessment}

Although all models develop spontaneous neuronal activity and (network) bursting, a culture with astrocytes increase synchronicity (see above), mimics the in vivo brain more closely and thus has a clear advantage for neurotoxicity screening. Therefore, we used the neuronal co-cultures with astrocytes for a preliminary neurotoxicity assessment (Fig. 7A-C).

PTX $(10 \mu \mathrm{M})$, a widely used seizurogenic reference compound, shows limited effects on MSR in both models following acute exposure. However, PTX induces a decrease in MBR in the iCell ${ }^{\circledR}$ Glutaneuron-Astrocyte co-culture, whereas it significantly increases MBR in the iCell ${ }^{\circledast}$ Glutaneuron-iCell ${ }^{\circledast}$ neuron-Astrocyte co-culture (Fig. 7A). The neurotoxic insecticide endosulfan ( $1 \mu \mathrm{M})$, a $\mathrm{GABA}_{\mathrm{A}}$ receptor antagonist, has limited effects on the MSR in the iCell ${ }^{\oplus}$ GlutaneuronAstrocyte co-culture, whereas it increases the MBR of this model. On the other hand, endosulfan exposure significantly reduces MSR in the iCell ${ }^{\circledR}$ GlutaneuroniCell ${ }^{\circledast}$ neuron-Astrocyte co-culture, but shows no increase in MBR (Fig. 7B). Finally, the known neurotoxicant $\mathrm{MeHg}(30 \mu \mathrm{M})$ has no major effects on MSR for both models (Fig. 7 C). MBR is reduced in both models, although significance is reached only for the iCell ${ }^{\circledR}$ Glutaneuron-Astrocyte co-culture.

The difference in chemical sensitivity between the iCell ${ }^{\circledR}$ Glutaneuron-Astrocyte co-culture and the iCell ${ }^{\circledR}$ Glutaneuron-iCell ${ }^{\circledR}$ neuron-Astrocyte co-culture is already apparent from the changes in MSR and MBR. Inclusion of additional metric parameters in a heat map further illustrates these distinctions (Fig. 8).

PTX affects both models differently; where most parameters show a decrease in the iCell ${ }^{\circledR}$ Glutaneuron-Astrocyte co-culture, an increase is visible in the iCell ${ }^{\circledR}$ Glutaneuron-iCell ${ }^{\circledR}$ neuron-Astrocyte co-culture. Similarly, endosulfan changes most of the metric parameters for both co-cultures. Some parameters increase in both models, such as network burst frequency, whereas for most burst-related parameters an opposite effect is seen. Finally, MeHg show a signature that is distinct from those of PTX and endosulfan, although the differences between the two tested co-cultures are less pronounced for MeHg. Overall, these neurotoxicity assessment metrics show that considerable differences exist between both models with respect to chemical sensitivity. 

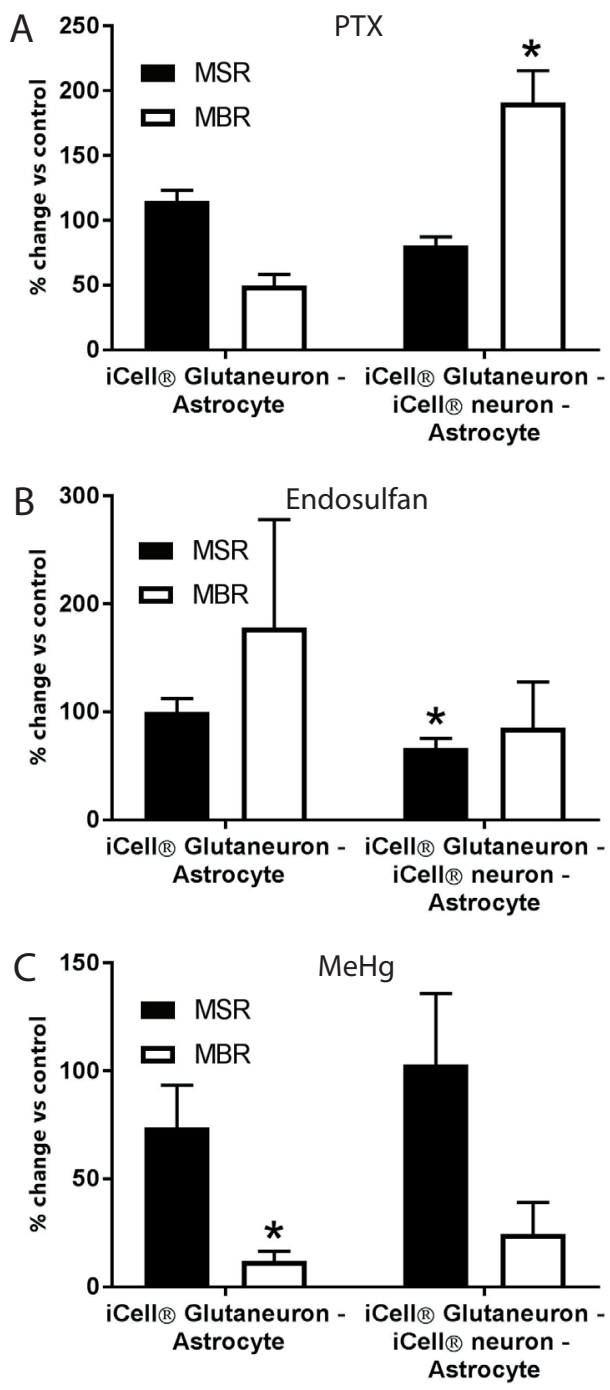

Figure 7. Toxicological modulation of spontaneous activity and bursting of iCell ${ }^{\circledR}$ GlutaneuronAstrocyte co-culture and iCell ${ }^{\circledast}$ Glutaneuron-iCell ${ }^{\circledast}$ neuron-Astrocyte co-culture. Cultures were exposed at DIV21 to PTX (10 $\mu \mathrm{M}$; A), endosulfan ( $1 \mu \mathrm{M} ; \mathrm{B})$ or MeHg (30 $\mu \mathrm{M} ; \mathrm{C})$. Data are expressed in MSR or MBR as \% of control; mean \pm SEM from $n=5-15, N=4(A), n=3-10, N=2$ (B) and $n=$ $3-9, \mathrm{~N}=2(\mathrm{C}) ;{ }^{*} \mathrm{p}<0.05$. 


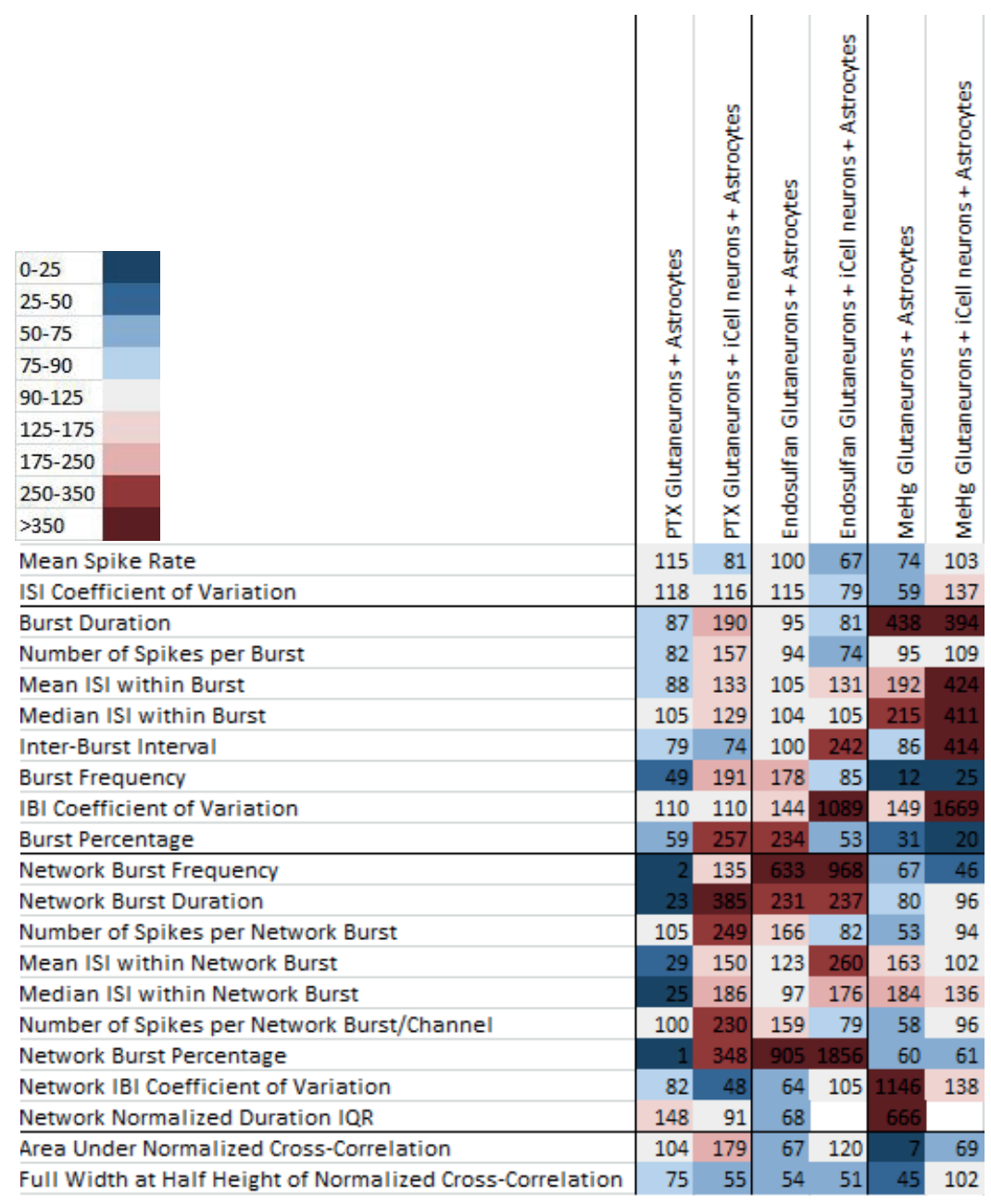

Figure 8. Heat map of the effects of PTX, endosulfan and MeHg on selected metric parameters on iCell ${ }^{\oplus}$ Glutaneuron-Astrocyte co-cultures and iCell ${ }^{\oplus}$ Glutaneuron-iCell ${ }^{\circledR}$ neuron-Astrocyte cocultures. Values in cells are means, expressed as $\%$ of control from $\mathrm{n}=3-15 ; \mathrm{N}=2-4$. Colour scaling is based on the magnitude of the \% of change relative to control. For empty cells no average could be calculated. 


\section{DISCUSSION}

Our immunocytochemistry data demonstrate the homogeneous neuronal nature of the iCell ${ }^{\circledR}$ Glutaneuron monoculture and iCell ${ }^{\circledR}$ Glutaneuron-iCell ${ }^{\circledR}$ co-culture (Fig. 1). Despite the absence of supportive cells, such as astrocytes, these cultures develop into neuronal networks that over time become spontaneously active (Figs. 3-4). These cultures already show neuronal activity and bursting as early as DIV4, which remains fairly stable over time (DIV21; Fig. 4A-B). The development of network burst activity, however, shows a time-dependence with an increase after $\sim 2$ weeks of culture (Fig. 4 C).

Since it is known that astrocytes play an important role in network formation, maturation function (Clarke and Barres, 2013) and protecting neurons from chemical-induced neurotoxicity (Takemoto et al., 2015; Wu, Yang, et al., 2017), we added astrocytes to our models to more closely resemble the in vivo brain. The co-cultures containing astrocytes are more heterogeneous (Fig. 2) and the complexity of the networks increases. Our mwMEA recordings show that co-cultures containing astrocytes, comparable with pure neuronal models, develop spontaneous neuronal activity as early as DIV4, with increased bursting behaviour following prolonged culture (DIV14-21; Figs. 3-5). The iCell ${ }^{\oplus}$ Glutaneuron-iCell ${ }^{\oplus}$ neuron-Astrocyte co-culture shows less active bursting wells than the iCell ${ }^{\circledast}$ Glutaneuron-Astrocyte co-culture, although the MSR and MBR are comparable between both cultures (Fig. 5). While the MNBR and the number of spikes in a network burst (Fig. 6D) are comparable between both astrocyte coculture models, the percentage of wells exhibiting network bursts was highest in the iCell ${ }^{\circledast}$ Glutaneuron-Astrocyte co-culture. Importantly, addition of astrocytes causes our astrocyte co-culture models to fire in a more synchronized manner. This increase in (synchronized) bursting activity by addition of astrocytes is in line with earlier findings (Amiri et al., 2013; Kuijlaars et al., 2016; Ishii et al., 2017). Such synchronized firing is thought to be an important feature for neuronal networks since it is has been suggested to be involved in cognitive and sensory processes (Engel et al., 2001; Ward, 2003). Overall, both our astrocyte co-cultures show a development comparable to that described for rat primary cortical neurons (Brown et al., 2016), with increasing MSR, MBR and number of spikes in network bursts during development (Fig. 5-6). 
As the two astrocyte co-culture models show a stable MSR, with a clear optimum for bursting activity, network burst activity and synchronized firing at DIV14-21, we used these two models to perform a preliminary neurotoxicity assessment at DIV21. Because we want our models to be able to detect seizures we choose two compounds for our preliminary neurotoxicity assessment that reportedly increase neuronal activity: PTX and endosulfan. The seizurogenic GABA receptor antagonists PTX causes an concentration-dependent increase in MSR in rat primary cortical cultures (Mack et al., 2014; Tukker et al., unpublished). However, exposure of the iCell ${ }^{\circledR}$ Glutaneuron-Astrocyte co-culture to PTX was without major effects on MSR and MBR (Fig. 7). Similarly, exposure of the iCell ${ }^{\circledast}$ Glutaneuron-Astrocyte co-culture to endosulfan is without major effects on MSR and MBR, whereas it causes a profound increase in MSR in rat primary cortical cultures (Dingemans et al., 2016). This apparent lack of chemosensitivity of the iCell ${ }^{\circledR}$ GlutaneuronAstrocyte co-culture could be a result of the high fraction of excitatory neurons, which results in a relatively high MSR and MBR that is not easily increased further following exposure to seizurogenic compounds. Alternatively, the apparent lack of chemosensitivity in the iCell ${ }^{\circledR}$ Glutaneuron-Astrocyte co-culture could be a direct result of the sparsity of GABAergic receptors, which may act as a specific target for PTX and endosulfan.

In line with these suggestions, the iCell ${ }^{\circledast}$ Glutaneuron-iCell ${ }^{\circledast}$ neuron-Astrocyte co-culture has a lower MBR than the iCell ${ }^{\circledast}$ Glutaneuron-Astrocyte co-culture, which is likely due to the larger fraction of inhibitory GABAergic neurons. Notably, exposure of the iCell ${ }^{\circledR}$ Glutaneuron-iCell ${ }^{\circledR}$ neuron-Astrocyte co-culture to PTX does result in an apparent increase in MBR, but without effect on MSR. Endosulfan lowers MSR, without affecting the MBR. These findings suggest that the effects of PTX and endosulfan may be distinguished by taking other metric parameters into account. Our heat map (Fig. 8) shows that indeed other parameters such as burst percentage, network burst duration and network burst percentage are affected in a stronger manner. Importantly, the chemosensitivity towards GABA receptor antagonists or seizurogenic compounds in general may thus critically depend on the fraction of inhibitory neurons in the network and/ or the degree of excitability prior to exposure. This would also explain some of the discrepancies reported earlier, e.g. Kuijlaars et al. (2016) report a decrease in bursting frequency on their iPSC-derived model following exposure to PTX. 
While the iCell $^{\circledR}$ Glutaneuron-iCell ${ }^{\circledR}$ neuron-Astrocyte co-culture is more sensitive to PTX and endosulfan than the iCell $^{\circledR}$ Glutaneuron-Astrocyte coculture, the latter is more responsive to MeHg. In rat primary cortical cultures, exposure to MeHg results in a profound decrease of MSR (Dingemans et al., 2016). At the concentrations chosen and limited exposure time $(30 \mathrm{~min})$ it is unlikely that the reported effects are confounded by acute cytotoxicity. This notion is further strengthened by the absence of cytotoxicity in rat primary cortical cultures (Dingemans et al., 2016; Tukker et al., unpublished; Yuan and Atchison, 2007; Yuan et al., 2005). Exposure of the iCell ${ }^{\circledR}$ Glutaneuron-Astrocyte co-culture to $\mathrm{MeHg}$ also causes a clear decrease in MBR, but is without effect in the iCell ${ }^{\circledR}$ Glutaneuron-iCell ${ }^{\circledR}$ neuron-Astrocyte co-culture. Since $\mathrm{MeHg}$ is expected to decrease neuronal activity, it is possible that culture models with a relatively high MSR and MBR are more sensitive towards neurotoxicants that reduce activity, contrary to the chemosensitivity towards seizurogenic compounds which seems to be higher in cultures with less excitation.

When the toxicity heat map data reported here (Fig. 8) is compared to published primary rat cortical data, we find that our iCell ${ }^{\circledR}$ Glutaneuron-Astrocyte coculture exposed to PTX exhibits a similar pattern (Bradley et al., 2018). Network burst duration, number of spikes per network burst, and burst duration all show an increase as compared to control, whereas the full width at half height shows a decrease. The PTX heat map of our iCell Glutaneuron ${ }^{\circledR}$ iCell neuron ${ }^{\circledR}$ Astrocyte co-culture model also matches with Bradley et al. (2018). Number of spikes per burst and median burst duration both show a decrease and the inter burst interval shows a decrease. However, the heat map pattern is clearly concentration-dependent (Bradley et al., 2018), which may also explain why our endosulfan heat map does not match their pattern.

In conclusion, we show that the commercially available hiPSC-derived models investigated in this study all form neuronal networks consisting of inhibitory GABAergic and excitatory glutamatergic neurons. These networks develop spontaneous neuronal activity and bursting. Addition of astrocytes to the cultures changes the activity pattern resulting in synchronized activity of the network. Despite the limited test set of compounds, our data already show that these models exert different responses following exposure to seizurogenic compounds and neurotoxicants. Our preliminary neurotoxicity assessment indicates that the sensitivity of hiPSC-derived neuronal models depends on the ratio of GABAergic and glutamatergic neurons, either because this affects the intrinsic degree of 
neuronal activity (MSR/MBR) or because it changes the degree of available target cells (GABA/glutamate receptors). Further characterization and toxicological validation of the different co-cultures models available are thus urgently needed. While currently hampered by the expense of hiPSCs, such validation should involve a more extensive set of reference compounds (including additional positive and negative controls that cover different modes of action) at multiple concentrations. Nevertheless, our preliminary data already provide a proof-of-principle for the usability of hiPSC-derived neuronal co-culture models for neurotoxicological screening in the mwMEA. A breakthrough of these hiPSC-derived neuronal models may enable animal-free neurotoxicity testing in the future, but may at present already be used as a prioritization tool prior to in vivo studies.

\section{CONFLICT OF INTEREST}

The authors declare that there are no conflicts of interest. Given his role as Editor in Chief of NeuroToxicology, Remco H.S. Westerink had no involvement in the peer-review of this article and has no access to information regarding its peerreview. Full responsibility for the editorial process for this article was delegated to Pamela J. Lein.

\section{ACKNOWLEDGEMENTS}

Members of the Neurotoxicology Research Group, Mimetas (Leiden, The Netherlands), Cellular Dynamics International (Madison, WI, USA) and the $\mathrm{NC} 3 \mathrm{R}$ team are gratefully acknowledged for helpful discussions. This work was funded by a grant from the National Centre for the Replacement, Refinement and Reduction of Animals in Research (NC3Rs; project number 50308-372160), the Netherlands Organisation for Health Research and Development (ZonMW; InnoSysTox project number 114027001) and by the Faculty of Veterinary Medicine (Utrecht University, The Netherlands). 


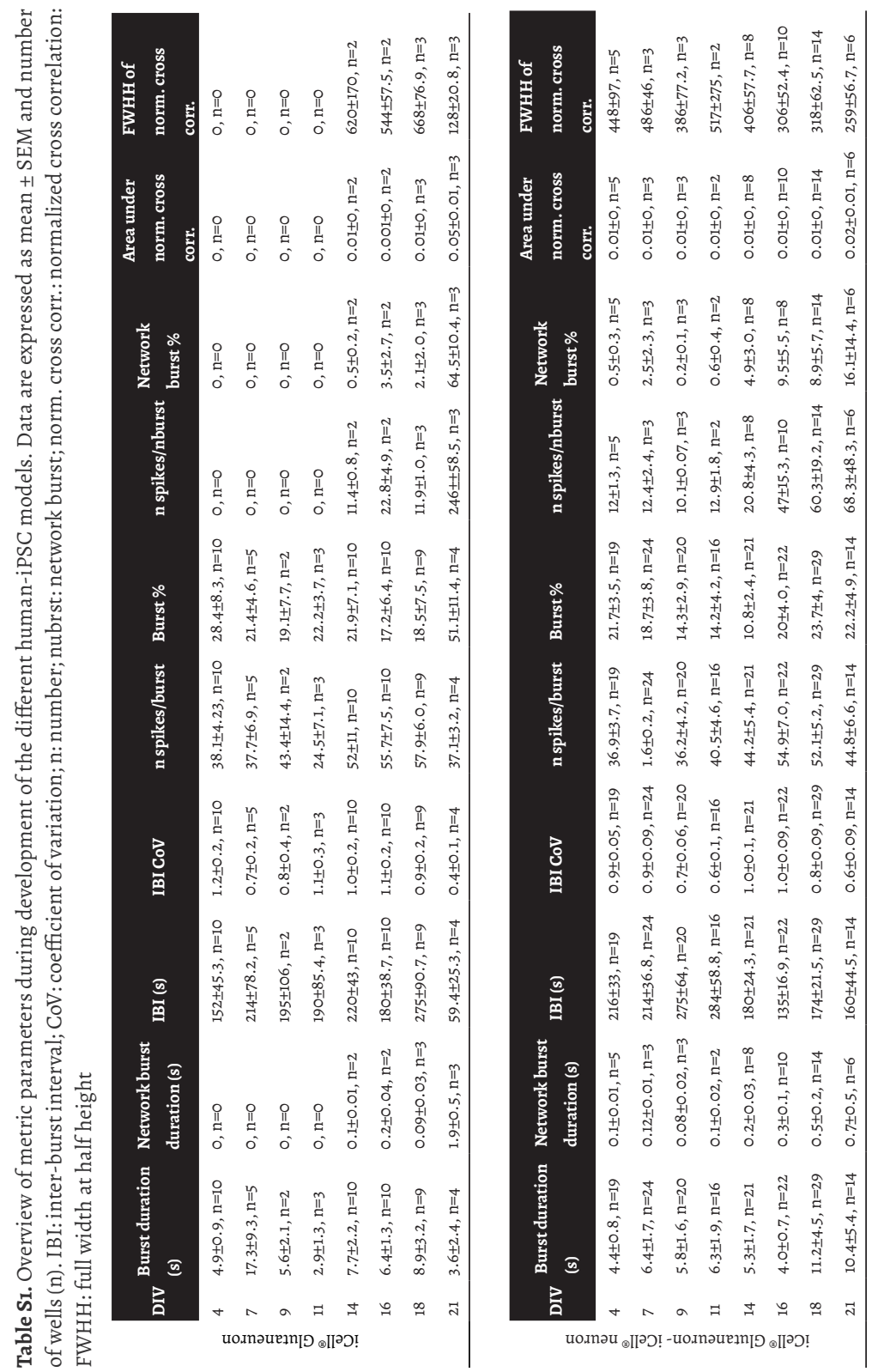




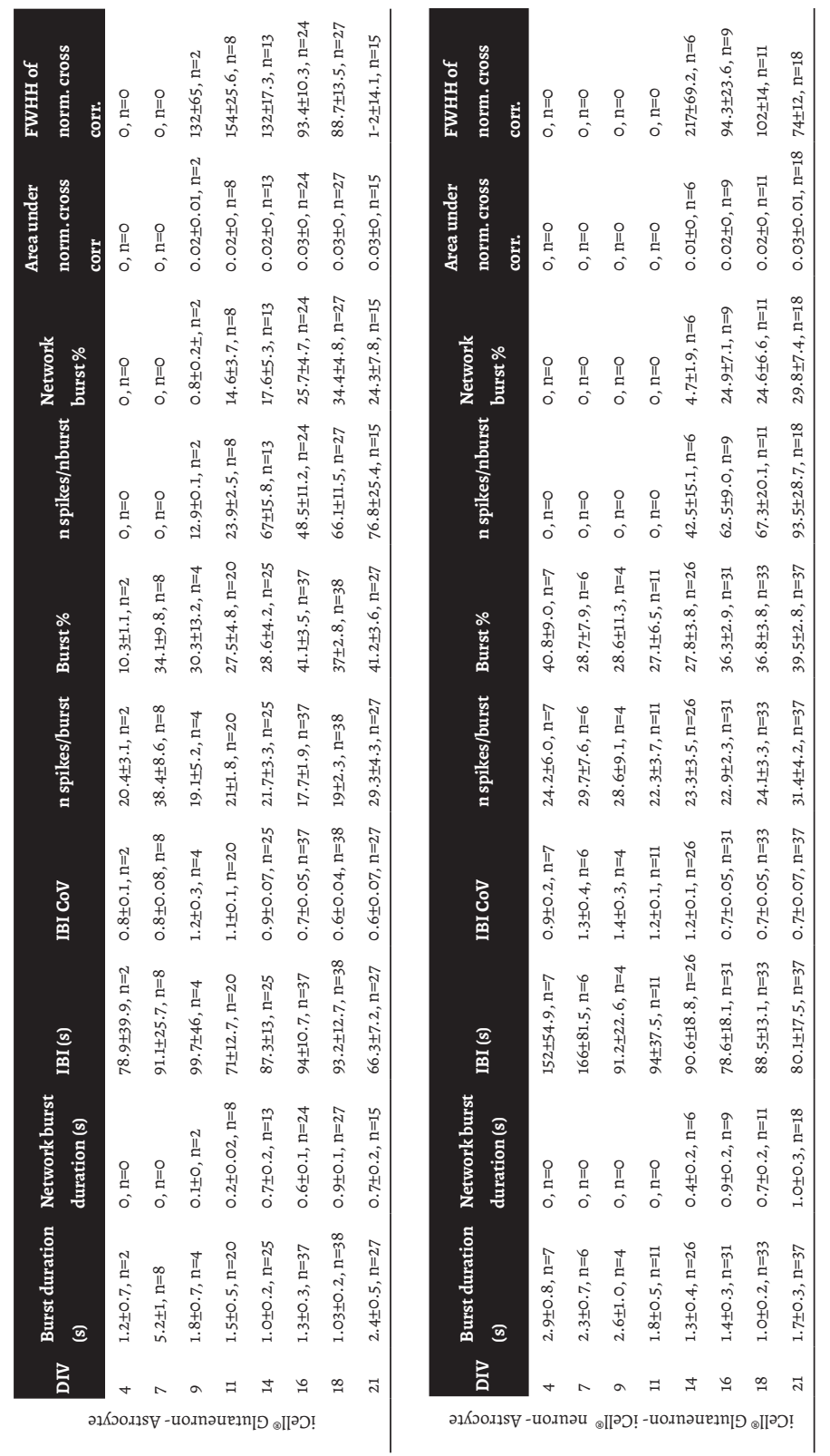




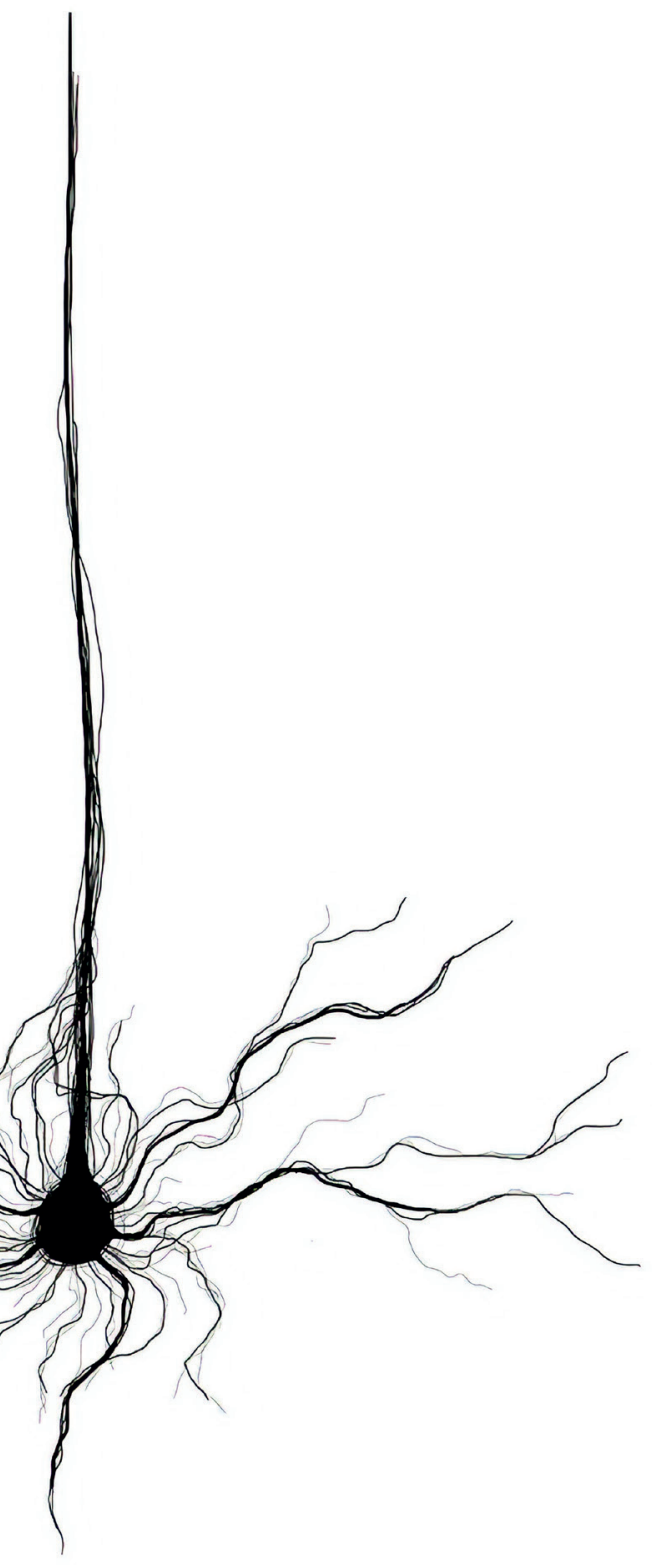


Part IV

\section{HUMAN IPSC-DERIVED} NEURONAL MODELS FOR IN VITRO SEIZURE LIABILITY ASSESSMENT 
Chapter 7

Towards animal-free neurotoxicity screening: applicability of hiPSC-derived neuronal models for in vitro seizure liability assessment

Anke M. Tukker, Regina G.D.M. van Kleef, Fiona M.J. Wijnolts, Aart de Groot and Remco H.S. Westerink

Neurotoxicology Research Group, Toxicology Division, Institute for Risk Assessment Sciences (IRAS), Faculty of Veterinary Medicine, Utrecht University, P.O. Box 80.177, NL-3508 TD Utrecht, The Netherlands.

ALTEX (2020), volume 37, pp 121-135

DOI:10.14573/altex.1907121

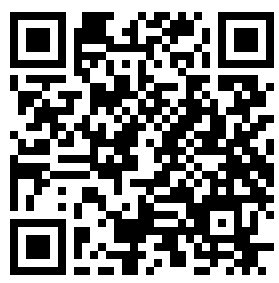




\begin{abstract}
A sizeable proportion of drug attrition is due to drug-induced seizures. Current available animal models frequently fail to predict human seizure liability. Therefore, there is a clear need for in vitro alternatives, preferably based on human-derived neurons to circumvent interspecies translation. The increasing number of commercially available human induced pluripotent stem cell (hiPSC)derived neuronal models holds great promise for replacing the widely accepted rodent primary cultures. We therefore tested three different hiPSC-derived neuronal models for their applicability for in vitro seizure liability assessment.
\end{abstract}

Using immunofluorescent stainings and multi-well micro-electrode arrays we show that all models develop functional neuronal networks that exhibit spontaneous activity and (network) bursting behaviour. Developmental patterns differ between the models, probably due to differences in model composition and seeding density. Nevertheless, neuronal activity and (network) bursting can be reproducibly modulated with the seizurogenic compounds strychnine, picrotoxin (PTX) and 4-aminopyridine (4-AP). However, the sensitivity and degree of chemical-induced effects differs between the models, which can likely be explained by differences in seeding density, maturation and different ratios of inhibitory and excitatory cell types. Importantly, compared to rat primary cortical neurons, the hiPSC-derived neuronal models were equally, or even better in the case of 4-AP, suited to detect seizurogenicity. Overall, our data indicate that hiPSC-derived neuronal models may in the future be used as a first screening tool for in vitro seizure liability assessment. However, before hiPSC-derived neuronal models can fully replace animal experiments, more compounds should be tested and the available models must be further characterised to fully understand their applicability.

Keywords: human induced pluripotent stem cell (hiPSC)-derived neuronal models, seizure liability assessment, alternatives to animal testing, micro-electrode array (MEA) 


\section{INTRODUCTION}

Drug development and safety testing are often done using in vivo and ex vivo experiments. These experiments are however not fully predictive for adverse effects in humans. While human drug approval rates have been rising in recent years, only $10.4 \%$ of drugs in phase I of drug development will ultimately gain final approval (Hay et al., 2014). Most drugs thus fail the trajectory, often due to safety concerns related to the central nervous system (CNS). Even when compounds reach the market, drug attrition as a result of neurotoxicity and CNS problems remains a challenging concern (Onakpoya et al., 2016). An increased risk of seizures, i.e., periods of abnormal, rhythmic and uncontrolled hyperexcitability of neurons characterised by hyper-synchronicity of electrical activity (Easter et al., 2009; Jiruska et al., 2013), is amongst the most encountered CNS related problems during pre-clinical drug development (Authier et al., 2016). Drug-induced seizures can be life threatening and are classified as severe adverse drug reactions. It is therefore of utmost importance that (increased) seizure liability can be detected early during drug development.

Since animal models frequently fail in human seizure liability predictions, their use is debated (Little et al., 2019). Consequently, in vitro alternatives are required to reduce the number of animal tests and to improve safety screening. However, in vitro seizure liability testing is challenging because the CNS contains many different cell types with a diverse range of drug targets that can all have effects on brain function and excitability (Easter et al., 2009). In vitro models for seizure liability screening should ideally thus model the in vivo brain as closely as possible (Westerink, 2013).

The most commonly used ex vivo model for seizure liability assessment in drug research is the brain slice assay (Authier et al., 2016). While the brain slice assay accurately mimics the in vivo organization of the brain (Grainger et al., 2018), including active and intact neuronal networks, these slices have a relatively short life-span (Buskila et al., 2015). More importantly, brain slices are still of animal origin and recordings require specific expertise and equipment, and are labour intensive, thereby limiting high-throughput screening (Grainger et al., 2018). The recent introduction of multiwell micro-electrode array (mwMEA) recordings eliminates some of these concerns. For this technique, cells are grown on a culture surface area with an integrated array of micro-electrodes allowing for non-invasive recordings of extracellular local field potentials simultaneously 
at different locations in the in vitro network (for review see Johnstone et al., 2010). MEA recording yield a broad range of data on parameters that describe the state of the network. Parameters related to activity and synchronicity can be used to detect a hyper-active and/or hyper-synchronised state of the network, linked to seizure like events in the in vivo situation (Ishii et al., 2017; Bradley et al., 2018). Many cell types can be grown on MEAs, but rat primary cortical cultures are the current gold standard (Authier et al., 2016). Primary cortical cultures grown on MEAs possess many characteristics of in vivo neurons, such as development of spontaneous network activity including (network) bursting (Cotterill, Charlesworth, et al., 2016) and responsiveness to neurotransmitters, pharmacological agents and toxicological modulation (Hogberg et al., 2011; McConnell et al., 2012; Nicolas et al., 2014; Valdivia et al., 2014; Hondebrink et al., 2016). Rodent cortical cultures grown on mwMEAs have been used for seizure liability assessment with positive results as known seizurogenic compounds increased spiking, (network) bursting activity and/or synchronicity (Bradley et al., 2018; Kreir et al., 2018; Fan et al., 2019). Although the combination of mwMEA and rodent primary cultures is promising, it is still based on animal cells.

As also outlined in NC3R's Crack-It Neuratect Challenge (http://www.NC3Rs.org. $\mathrm{uk}$ ), there is a clear need for new in vitro drug screening models that are able to reliably detect seizures and can be used in a time and cost-efficient manner. These models should preferably be of human origin to circumvent interspecies translation and reduce the number of animal experiments. Human induced pluripotent stem cell (hiPSC)-derived neurons lack the ethical concerns of embryonic stem cells and animal experiments. It has been shown that hiPSC-derived neurons can be cultured on MEAs and exhibit spontaneous neuronal activity with (network) bursting behaviour of mature neurons (Odawara et al., 2016; Paavilainen et al., 2018; Sasaki et al., 2019). On top of that, these hiPSC-derived neurons can be modulated with known neurotoxicants and drugs (Tukker et al., 2016; Hondebrink et al., 2017; Odawara et al., 2018). However, one of the challenges in using hiPSC-derived neurons is the long time it takes to generate the neurons, which can vary from weeks (Kuijlaars et al., 2016) till even months (Odawara et al., 2016), and the potential variability between batches of differentiated cells (Little et al., 2019). The introduction of commercially available hiPSC-derived neurons can help to overcome these concerns as they can be purchased in large, quality-controlled quantities (Anson et al., 2011; Little et al., 2019) and can be used for neurotoxicity screening following a relatively short culture duration (Tukker et al., 2018). 
With the increasing availability of hiPSC-derived neurons, the differences between these models also increase. Human neuronal models can differ for example in the ratio of excitatory and inhibitory neurons as well as in the presence or absence of astrocytes. These factors greatly affect the (bursting) behaviour of the hiPSC-derived model (Tukker et al., 2018). Also, variations in the differentiation protocol as well as the level of maturation before freezing may affect the culture. Therefore, this research aims to explore whether these models, despite their differences, are suitable for animal-free seizure liability testing. To that aim, we cultured three different commercially available hiPSC-derived neuronal models to assess the development of spontaneous neuronal network activity. Subsequently, these models were challenged with known seizurogenic compounds and the results were compared to data from rat primary cortical cultures that were exposed in parallel.

\section{MATERIALS AND METHODS}

\subsection{Animals}

All experiments were conducted in accordance with the Dutch law and approved by the Ethical Committee for Animal Experiments of Utrecht University. Animals were treated humanely, and all efforts were made to alleviate suffering. Primary cultures of rat cortical neurons were prepared from postnatal day (PND) 0-1 pups of timed pregnant Wistar rat damns (Envigo, Horst, The Netherlands) as described in section 2.2.1.

\subsection{Chemicals}

Neurobasal ${ }^{\circ}$-A medium, Dulbecco's Modified Eagle Medium (DMEM)-F12, Foetal Bovine Serum (FBS), penicillin - streptomycin $(5000 \mathrm{U} / \mathrm{mL}-5000 \mu \mathrm{g} / \mathrm{mL}$ for rat primary cortical culture media and $10.000 \mathrm{U} / \mathrm{mL}-10.000 \mu \mathrm{g} / \mathrm{mL}$ for supplemented Brainphys $^{\mathrm{TM}}$ medium), B27 supplement, $\mathrm{N} 2$ supplement, L-glutamine, 4',6-diamidino-2-phenylindole (DAPI), donkey anti-rabbit Alexa Fluor 488 and donkey anti-mouse Alexa Fluor 594 were obtained from Life Technologies (Bleiswijk, The Netherlands). Goat anti-chicken Alexa Fluor 647 was obtained from Jackson ImmunoResearch Europe (Ely, UK). Paraformaldehyde (PFA) was obtained from Electron Microscopy Sciences (Hatfield, Pennsylvania, USA). Rabbit anti- $\beta$ (III)-tubulin (Ab18207), mouse anti-S10o $\beta$ (Ab11178) and chicken anti-MAP2 (Ab5392) were obtained from Abcam (Cambridge, United Kingdom). 
FluorSave was obtained from Calbiochem (San Diego, California, USA). iCell ${ }^{\circ}$ Neural Supplement B and Nervous System Supplement were provided by Cellular Dynamics International (Madison, WI, USA). BrainPhys ${ }^{\mathrm{TM}}$ neuronal medium was obtained from StemCell Technologies (Cologne, Germany). Neuro.4U ${ }^{\circ}$ basal medium A and CNS. $4 \mathrm{U}^{\circ}$ supplement were provided by Ncardia (Leiden, The Netherlands). SynFire seeding basal medium, short-term basal medium, long-term basal medium and accessory supplements were provided by NeuCyte (Sunnyvale, CA, USA). Laminin (L2020), 50\% polyethyleneimine (PEI) solution, sodium borate, boric acid and all other chemicals (unless stated otherwise) were obtained from Sigma-Aldrich (Zwijndrecht, The Netherlands).

Stock solutions of strychnine $\mathrm{HCl}$ were made in purity-checked dimethyl sulfoxide (DMSO) and stored at $4^{\circ} \mathrm{C}$. 4-Aminopyridine (4-AP) was freshly dissolved in medium at the day of experiments. Stock solutions of picrotoxin (PTX) were made in ethanol (EtOH; VWR, Amsterdam, The Netherlands) on the day of the experiment. Final concentration of solvent in exposure solutions was always kept at or below $0.1 \%$ ( $\mathrm{vol} / \mathrm{vol})$.

\subsection{Cell culture}

Primary rat cortical cultures and all hiPSC-derived neuronal co-cultures were kept at $37^{\circ} \mathrm{C}$ in a humidified $5 \% \mathrm{CO}_{2}$ incubator. 48 -Wells MEA plates (Axion BioSystems Inc., Atlanta, GA, USA) and $\mu$-slide 8-well chambered coverslips (Ibidi $\mathrm{GmbH}$, Planegg, Germany) were pre-coated with $0.1 \%$ PEI solution diluted in borate buffer ( $24 \mathrm{mM}$ sodium borate $/ 50 \mathrm{mM}$ boric acid in Milli- $\mathrm{Q}, \mathrm{pH}$ adjusted to 8.4). See Table 1 for an overview of the culture details of the four different models, including the day at which their chemical sensitivity was assessed (exposure DIV). Notably, the exposure days used to determine the drug response differs between hiPSC-derived neuronal models as a consequence of manufacturer's recommendations. Nevertheless, all models did show a mature network phenotype with (network) bursting on the day of exposure.

\subsection{1 iCell $^{\circ}$ Glutaneurons - iCell ${ }^{\circ}$ Astrocytes co-culture}

iCell $^{\circ}$ Glutaneurons (Lot\# 103288; Cellular Dynamics International, Madison, WI, USA) and iCell ${ }^{\circ}$ Astrocytes (Lot\# 11493, 1414 and 1444; Cellular Dynamics International, Madison, WI, USA) were thawed and cultured according to manufacturer's protocol. Briefly, each cell type was thawed separately in supplemented BrainPhys ${ }^{\mathrm{TM}}$ medium (BrainPhys $\mathrm{s}^{\mathrm{TM}}$ medium supplemented with $2 \%$ iCell $^{\circ}$ Neural Supplement B, $1 \%$ Nervous System Supplement, $1 \%$ N2, $1 \%$ penicillin 
- streptomycin and $0.1 \%$ laminin). The cell pellet was diluted in dotting medium (supplemented BrainPhys ${ }^{\mathrm{TM}}$ medium with $10 \%$ laminin) at a density of $15 \mathrm{k}$ cells $/ \mu \mathrm{L}$ for iCell ${ }^{\circ}$ Glutaneurons or $6,6 \mathrm{k}$ cells $/ \mu \mathrm{L}$ for iCell ${ }^{\circ}$ Astrocytes. Before plating, iCell ${ }^{\circ}$ Glutaneurons and iCell $^{\circ}$ Astrocytes were premixed into a co-culture containing $120 \mathrm{k} \mathrm{iCell}{ }^{\circ}$ Glutaneurons $(85 \%)$ and $20 \mathrm{k} \mathrm{iCell}^{\circ}$ Astrocytes $(15 \%)$ and then plated in $11 \mu \mathrm{L}$ droplets (140k cells/droplet) over the electrode field of pre-coated MEA wells or in a chamber of the $\mu$-slide coverslip. Cells were allowed to adhere for $\sim 1 \mathrm{hr}$ before $300 \mu \mathrm{L}$ (MEA) or $200 \mu \mathrm{L}$ (coverslip) room temperature (RT) supplemented BrainPhys ${ }^{\mathrm{TM}}$ medium was added. $50 \%$ Medium changes with RT supplemented BrainPhys $^{\mathrm{TM}}$ medium took place at DIV1, 2, 4, 6, 8, 10, 12 and 14 .

\subsubsection{CNS. $4 U^{\circ}$ culture}

CNS.4U (Lot\# CN39CL_V_2M and CN59CL_V-x2; Ncardia, Leiden, The Netherlands) were obtained as a mixture of $\sim 40 \%$ glutamatergic neurons, $\sim 40 \%$ GABAergic neurons, $\sim 10 \%$ dopaminergic neurons and $\sim 10 \%$ astrocytes. Cells were thawed and cultured according to manufacturer's protocol. Briefly, a vial was thawed in Neuro. $4 \mathrm{U}^{\circ}$ basal medium A and the cell pellet was dissolved in complete Neuro. $4 \mathrm{U}^{\circ}$ medium A (basal medium and supplement) at a density of $12 \mathrm{k}$ cells/ $\mu \mathrm{L}$. Cells were plated in $3 \mu \mathrm{L}$ droplets ( $36 \mathrm{k}$ cells in total) directly over the electrode field of pre-coated MEA wells or in the chamber of a $\mu$-slide coverslip. Cells were allowed to adhere for $\sim 1 \mathrm{hr}$ before $300 \mu \mathrm{L}$ (MEA) or $200 \mu \mathrm{L}$ (coverslip) RT Neuro. $4 \mathrm{U}^{\circ}$ medium was added. At DIV1, 7, 14 and 21, 100\% medium changes were performed with complete Neuro. $4 \mathrm{U}^{\circ}$ medium. At DIV2, 4, 9, 11, 16, 18, 50\% of the medium was replaced with complete Neuro. $4 \mathrm{U}^{\oplus}$ medium.

\subsubsection{SynFire ${ }^{\circledast}$ iNs co-culture}

SynFire glutamatergic neurons (Lot\#104, 109 and 116), SynFire GABAergic neurons (Lot\#105, 109, 110 and 116) and Synfire astrocytes (Lot\#12854 and 13029; all from NeuCyte, Sunnyvalle, CA, USA) were thawed and cultured according to manufacturer's protocol. In short, each cell type was thawed separately in DMEM-F12 medium. The cell pellet was dissolved in complete seeding medium (containing seeding supplement) at a density of $10 \mathrm{k}$ cells/ $\mu \mathrm{L}$ (for all cell types). Next, a mixture was made containing 140k glutamatergic neurons ( $52 \%$ of total cell number), $60 \mathrm{k}$ GABAergic neurons (22\%) and 70k astrocytes (26\%). The mixture was plated in $50 \mu \mathrm{L}$ droplets ( $270 \mathrm{k}$ cells in total) over the electrode field of precoated MEA wells or in a chamber of the $\mu$-slide coverslip. Cells were left overnight to adhere. Next day (DIV1), $250 \mu \mathrm{L}$ (MEA) or $150 \mu \mathrm{L}$ (coverslip) RT complete shortterm maintenance medium (containing short-term supplement) was added. 
At DIV3 and 5, 50\% medium changes with complete short-term maintenance medium took place. The remaining 50\% medium changes at DIV7, 10, 13, 16, 19, 22 and 25 were performed with RT complete long-term maintenance medium (containing long-term supplement A and B).

Table 1. Composition, density and exposure DIV of the different neuronal culture models.

\begin{tabular}{|c|c|c|c|c|}
\hline Cell models & Cell types (\%) & $\begin{array}{l}\text { Excitatory: inhibitory } \\
\text { neurons (ratio) }\end{array}$ & $\begin{array}{l}\text { Seeding } \\
\text { density }\end{array}$ & $\begin{array}{l}\text { Exposure } \\
\text { DIV }\end{array}$ \\
\hline $\begin{array}{l}\text { iCell }^{\circledR} \text { Glutaneurons - iCell } \\
\text { Astrocytes co-culture }\end{array}$ & $\begin{array}{l}\text { Neurons ( } 85 \% \text {, of which } \\
70 \% \text { glutamatergic and } 30 \% \\
\text { GABAergic) / Astrocytes ( } 15 \% \text { ) }\end{array}$ & $2.3: 1$ & $140 \mathrm{k}$ & DIV14 \\
\hline CNS.4U $\mathrm{U}^{\circledast}$ co-culture & $\begin{array}{l}\text { Glutamatergic neurons }(\sim 40 \%) \\
\text { / GABAergic neurons }(\sim 40 \%) / \\
\text { Dopaminergic neurons }(\sim 10 \%) / \\
\text { Astrocytes }(\sim 10 \%)^{1}\end{array}$ & 1:1 & $36 \mathrm{k}$ & DIV23 \\
\hline SynFire ${ }^{\circledast}$ iNs co-culture & $\begin{array}{l}\text { Glutamatergic neurons (52\%) } \\
\text { / GABAergic neurons (22\%) / } \\
\text { Astrocytes ( } 26 \%)\end{array}$ & $2.3: 1$ & $270 \mathrm{k}$ & DIV28 \\
\hline $\begin{array}{l}\text { Rat primary cortical } \\
\text { neurons }\end{array}$ & $\begin{array}{l}\text { Neurons }(55 \% \text {, of which } 70-80 \% \\
\text { glutamatergic and } 20-30 \% \\
\text { GABAergic }^{2} \text { ) / Astrocytes }(45 \%)^{3}\end{array}$ & $3: 1$ & $100 \mathrm{k}$ & DIV9-11 \\
\hline
\end{tabular}

\subsubsection{Rat primary cortical culture}

Primary rat cortical cells were isolated from PNDO-1 Wistar rat pups as described previously (Dingemans et al., 2016; Tukker et al., 2016). Briefly, PNDo-1 pups were decapitated and cortices were rapidly dissected on ice and kept in dissection medium (Neurobasal ${ }^{\circ}$-A supplemented with $25 \mathrm{~g} / \mathrm{L}$ sucrose, $450 \mu \mathrm{M}$ L-glutamine, $30 \mu \mathrm{M}$ glutamate, $1 \%$ penicillin/streptomycin and 10\% FBS, $\mathrm{pH} 7.4$ ) during the entire procedure. Cortices were dissociated to a single-cell suspension by mincing with scissors, trituration and filtering through a $100 \mu \mathrm{m}$ mesh (EASYstrainer, Greiner). The cell suspension was diluted to $2 \times 10^{6}$ cells $/ \mathrm{mL}$. Droplets of $50 \mu \mathrm{L}$ (10ok cells in total) were placed on the electrode fields in wells of pre-coated MEA plates. Cells were left to adhere for $\sim 2 \mathrm{hr}$ before adding $450 \mu \mathrm{L}$ dissection medium. At DIV2, $90 \%$ of the dissection medium was replaced with glutamate medium (Neurobasal ${ }^{\circ}$-A supplemented with $25 \mathrm{~g} / \mathrm{L}$ sucrose, $450 \mu \mathrm{M}$ L-glutamine, $30 \mu \mathrm{M}$ glutamate, $1 \%$ penicillin/streptomycin and $2 \% \mathrm{~B} 27$ supplement, $\mathrm{pH} 7.4$ ) to prevent glial overgrowth. At DIV4 $90 \%$ of the glutamate medium was replaced with FBS medium (Neurobasal ${ }^{\circ}$-A supplemented with $25 \mathrm{~g} / \mathrm{L}$ sucrose, $450 \mu \mathrm{M}$ 
L-glutamine, $1 \%$ penicillin/streptomycin and 10\% FBS, $\mathrm{pH} 7.4$ ).

\subsection{Immunocytochemistry}

The $\mu$-slide coverslips with different hiPSC-derived neuronal co-cultures were fixated on the day of exposure with 4\% PFA in $0.1 \mathrm{M} \mathrm{PBS}$ ( $\mathrm{pH} 7.4$ ) for $15 \mathrm{~min}$ at RT. Following fixation, coverslips were quenched for PFA, permeabilized and incubated for $20 \mathrm{~min}$ at $\mathrm{RT}$ with $20 \mathrm{mM} \mathrm{NH}_{4} \mathrm{Cl}$ in blocking buffer $(2 \%$ bovine serum albumin and $0.1 \%$ saponin in PBS) as described previously (Tukker, Wijnolts, et al., 2019). Hereafter, coverslips were incubated overnight at $4^{\circ} \mathrm{C}$ with rabbit anti$\beta$ III tubulin (final dilution 1:500), mouse anti-S100 $\beta$ (final dilution 1:500) and chicken anti-MAP2 (final dilution 1:1000) in blocking buffer. Next, coverslips were washed thrice with blocking buffer and incubated with donkey anti-rabbit Alexa Fluor $^{\circledR} 488$, donkey anti-mouse Alexa Fluor 594 and goat anti-chicken Alexa Fluor 647 (final dilution 1:100) for $45 \mathrm{~min}$ at RT in the dark. During the last 2-3 min of this incubation $200 \mathrm{nM}$ DAPI for nuclear staining was added. Coverslips were again washed thrice with blocking buffer and sealed with 1-2 drops of FluorSave. The coverslips were stored in the dark at $4^{\circ} \mathrm{C}$ until further use. Immunostained coverslips were visualised with a Leica SPEII Confocal microscope (Leica DMI4000 equipped with TCS-SPE-II) using a 40x oil immersion objective (ACS APO IMM NA 0.6). Images were captured as .lif files using Leica Application Suite Advanced Fluorescence software (LAS AF version 2.6.0; Leica Microsystems $\mathrm{GmbH}$, Wetzlar, Germany).

\subsection{MEA measurements}

Each well of a 48-well MEA plate contains 16 nanotextured gold micro-electrodes $(\sim 40-50 \mu \mathrm{m}$ diameter; $350 \mu \mathrm{m}$ spacing) with 4 integrated ground electrodes yielding a total of 768 channels that can simultaneously be recorded. Spontaneous electrical activity was recorded on the days of medium changes and on the day of exposure (Table 1) as described previously (Nicolas et al., 2014; Tukker, Wijnolts, et al., 2019). In short, signals were recorded using a Maestro 768-channel amplifier with integrated heating system and temperature controller and a data acquisition interface (Axion BioSystems Inc., Atlanta, GA, USA). Data acquisition was managed with Axion's Integrated Studio (AxIS 2.4.2.13) and recorded as .RAW files. All channels were sampled at the same time with a gain of $1200 \mathrm{x}$ and a sampling frequency of $12.5 \mathrm{kHz}$ / channel with a $200-5000 \mathrm{~Hz}$ band-pass filter. Prior to the recording, MEA plates were allowed to equilibrate for $\sim 10 \mathrm{~min}$ in the Maestro. 
Table 2. Overview and description of different metric parameters. Adapted from Tukker et al., 2018.

\section{Metric parameter}

Mean spike rate (MSR)

ISI coefficient of variation

Number of active electrodes

Number of active wells

Mean burst rate (MBR)

Number of bursting electrodes

Number of bursting wells

Burst duration

Number of spikes per burst

Mean inter-spike interval (ISI) within burst

Inter-burst interval (IBI)

IBI coefficient of variation

Burst percentage

Mean network burst rate (MNBR)

Network burst duration

Number of spikes per network burst

Mean ISI within network burst

Number of electrodes participating in network burst

Number of network bursting wells

Network burst percentage

\section{Description}

Total number of spikes divided by recording time $(\mathrm{Hz})$

Standard deviation ISI (time between spikes) divided by the mean ISI. Measure for spike regularity: 0 indicates perfect spike distribution, >1 signals bursting Average number of electrodes with a spike rate of at least 0.1 spike/s

Number of wells that exhibits activity that meets spike criteria

Total number of bursts divided by recording time $(\mathrm{Hz})$ Average number of electrodes in the well with number of bursts/second that is higher than the burst criterion of 0.005 bursts/s

Number of wells that meets the bursting criteria Average time from the first spike in a burst till the last spike (s)

Average number of spikes occurring in a burst

Mean inter-spike interval within a burst (s)

Time between the last spike of a burst and the first spike of a subsequent burst (s)

Standard deviation of IBI divided by the mean IBI. Measure for burst regularity

Percentage of total number of spikes occurring in a burst Total number of network bursts divided by recording time $(\mathrm{Hz})$

Average time from the first spike till the last spike in a network burst (s)

Average number of spikes occurring in a network burst Average of the mean ISIs within a network burst (s) Average number of electrodes with spikes that participate in the network burst

Number of wells that meets the network burst criteria Percentage of total spikes occurring in a network burst 
Network IBI coefficient of variation

Network normalised duration IQR

Area under normalised cross-correlation

Full width at half height (FWHH) of

normalised cross-correlation
Standard deviation of network IBI divided by the mean network IBI. Measure of network burst rhythmicity: value is small when bursts occur at regular interval and increases when bursts occur more sporadic Interquartile range of network bursts durations. Measure for network burst duration regularity: larger values indicate wide variation in duration.

Area under inter-electrode cross-correlation normalised to the auto-correlations. The higher the value, the greater the synchronicity of the network.

Width at half height of the normalised cross-correlogram. Measure for network synchrony: the higher the value, the less synchronised the network is.

To determine effects of the known seizurogenic compounds PTX, 4-AP and strychnine on spontaneous activity (spiking and (network) bursting behaviour) of the different cell cultures, activity was recorded prior to exposure to generate a baseline recording. Immediately following this recording, cells were exposed to the seizurogenic compounds or the appropriate solvent control and activity was recorded for another $30 \mathrm{~min}$ plus the time it took to expose all wells. Concentrations tested were determined by the NC3R CRACK-it team based on the list from the HESI NeuTox MEA Subteam, and were in line with earlier studies assessing seizure liability (Kreir et al., 2018). Each well was exposed to only one single concentration of one compound in order to prevent receptor (de)sensitisation or other compensatory cellular processes. For each experimental condition, MEA plates from at least two different plating rounds or culture preparations were used.

\subsection{Data analysis and statistics}

To determine (modulation of) spontaneous activity, .RAW data files were rerecorded to obtain Alpha Map files. In this re-recording, spikes were detected with the AxIS spike detector (Adaptive threshold crossing, Ada BandFIt v2) and a variable threshold spike detector set at 7x (rat primary cortical cultures) or 5.5x (all hiPSCderived neuronal cultures) standard deviation (SD) of internal noise level (rms) on each electrode. Post/pre-spike duration was set to 3.6/2.4 ms respectively. For further data analysis, spike files were loaded in NeuralMetric Tool (version 2.2.4, Axion BioSystems) and only active electrodes (MSR $\geq 0.1$ spikes/s) in active wells ( $\geq$ 1 active electrode) were included in data analysis. Bursts were extracted with the 
Poisson Surprise method (Legéndy and Salcman, 1985) with a minimal surprise of 10 and a minimum bursting frequency of 0.005 burst/s. Network bursts were determined with an adaptive threshold algorithm.

For developmental curves full recordings from the days of medium change and the baseline recording of the day of exposure were used for data analysis and the means of different network parameters (Table 2.) were calculated based on well averages. The percentage of active wells depicts the percentage of wells that fulfil the activity criteria relative to the total number of seeded wells. The percentage of busting wells depicts the percentage of wells that meet the bursting criteria and is calculated with respect to the number of active wells. Finally, the percentage of network bursting wells is the percentage of wells that exhibits network bursts relative to the number of bursting wells. This implicates that only the most active wells are included in the network burst developmental analysis, automatically resulting in a relatively high number of network bursting electrodes.

The effects of seizurogenic compounds on spontaneous activity were determined by comparing the baseline activity with activity following exposure. A custom-made MS Excel macro was used to calculate treatment ratios (TR) per well for the different metric parameters (Table 2) by: (parameter ${ }_{\text {exposure }} /$ parameter $_{\text {baseline }}$ ) x 100\%. Hereafter, TRs were normalised to appropriate vehicle control (medium, DMSO or EtOH). To prevent inclusion of exposure artefacts, effect analysis was performed in the window of 20-30 min post-exposure for all tested compounds.

Wells that showed an effect two times SD above or below average were considered outliers and removed for further data analysis ( $4 \%$ for iCell ${ }^{\circ}$ Glutaneurons - iCell $^{\circ}$ Astrocytes co-culture; $3 \%$ for CNS. $4 \mathrm{U}^{\circ}$ co-culture; $4 \%$ for SynFire ${ }^{\bullet}$ iNs co-culture and $5 \%$ for rat primary cortical culture). Concentration-dependent effects were determined by a one-way Welch ANOVA followed by a post hoc Dunnett test. $P$-values $<0.05$ were considered statistically significant. All statistical analyses were performed in $\mathrm{R}$ version 3.6.0 ( $\mathrm{R}$ core team 2019) using the DescTools package (by Signorell et al., 2019, version 0.99.28). Spider plots and heat maps were also created in $\mathrm{R}$ with the packages fmsb (by Nakazawa, 2018, version 0.6.3) and pheatmap (by Kolde, 2019, version 1.0.12) respectively. All data are presented as mean \pm standard error of the mean (SEM) from the number of wells (n) indicated, derived from at least 2 independent plating rounds $(\mathrm{N})$. 


\section{RESULTS}

\subsection{Immunofluorescent stainings of the different hiPSC-derived neuronal models}

As a first characterisation, the three different hiPSC-derived neuronal co-cultures were labelled with microtubule-associated protein 2 (MAP2, marker for mature neurons), $\beta($ III)tubulin (general neuronal marker) and S100 calcium binding protein $\beta$ (S10o $\beta$, marker for astrocytes) antibodies to visualise the heterogeneity and complexity of the in vitro networks. The iCell ${ }^{\circ}$ Glutaneurons - iCell ${ }^{\circ}$ Astrocytes co-culture (A), CNS. $4 \mathrm{U}^{\circ}$ co-culture (B) and SynFire iNs co-culture (C) all show network formation with a high degree of complexity at their corresponding days of exposure (Table 1). Images also indicate that neurons and astrocytes are spread evenly through the culture.

These results show that all three different hiPSC-derived neuronal co-cultures consist of mature neurons and astrocytes. The data also indicate that neuronal networks are formed. The network complexity and heterogeneity are comparable to the degree of the complexity present in the rat primary cortical culture (Hondebrink et al., 2016). These data thus suggest that all models may exhibit spontaneous network activity.
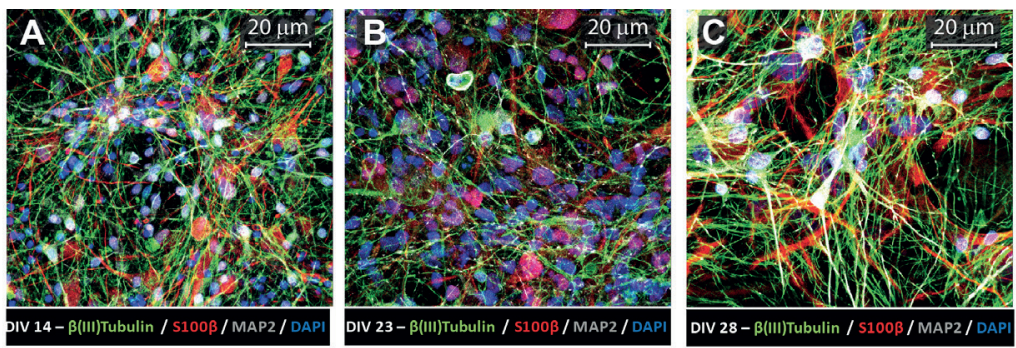

Figure 1. Immunofluorescent images of iCell $^{\circ}$ Glutaneurons - iCell $^{\circ}$ Astrocytes co-culture (A), CNS.4U $\mathrm{U}^{\circ}$ co-culture (B) and SynFire iNs co-culture (C) illustrating (neuronal) network structures with MAP2 (mature neurons; grey), $\beta(\mathrm{III})$ tubulin (general neuronal marker; green) and S100 $\beta$ (astrocytes; red). Nuclei are stained with DAPI (blue). Scale bar depicts $20 \mu \mathrm{m}$. 


\subsection{Development of spontaneous neuronal activity and (network) bursting behaviour}

To further test the applicability of these different hiPSC-derived neuronal models for seizure liability assessment, cells were cultured according to continuously improving manufacturer's recommendations up to DIV14 (iCell ${ }^{\circ}$ Glutaneurons - iCell ${ }^{\circ}$ Astrocytes co-culture), DIV23 (CNS.4U ${ }^{\circ}$ co-culture) or DIV 28 (SynFire iNs co-culture) on mwMEAs to assess the development of spontaneous neuronal network activity and bursting behaviour over time. All models develop spontaneous activity (Fig. 2). The mean spike rate (MSR) is relatively stable for the iCell $^{\circ}$ Glutaneurons - iCell $^{\circ}$ Astrocytes co-culture and $\mathrm{CNS}_{4} \mathrm{U}^{\circ}$ co-culture (Fig 2A/B, left), whereas MSR of the SynFire iNs co-culture increases during the entire culture period (Fig. $2 \mathrm{C}$, left). The percentage of spiking wells is stable and comparable $(>75 \%)$ for all models.

The mean burst rate (MBR) of the CNS. $4 \mathrm{U}^{\circ}$ co-culture is stable during the whole culture period, however the rate is low compared to the other two models, especially at the day of exposure (Fig. 2B, middle). The SynFire iNs co-culture has the highest MBR at the end of the culture period (Fig. $2 \mathrm{C}$, middle). The percentage of bursting wells is $>75 \%$ for all three models at the day of exposure.

At the beginning of the culture period, the $\mathrm{iCell}^{\circ}$ Glutaneurons - $\mathrm{iCell}^{\circ}$ Astrocytes co-culture has a high mean network burst rate (MNBR; Fig 2A, right), especially compared to the other two models (Fig. $2 \mathrm{~B} / \mathrm{C}$, right). However, this rate decreases rapidly over time to levels comparable with the other 2 models. At the day of exposure, all cultures exhibit a comparable MNBR, but the percentage of wells that exhibit network bursts is low in the CNS. $4 \mathrm{U}^{\circ}$ co-culture $(<50 \%)$ compared to the other two models ( $>75 \%)$.

Further analysis of additional (network) burst related parameters reveals additional differences between the models, as is depicted in spider plots (Fig. 3). It becomes clear from the lay-out of these graphs that the models develop in a different manner. Whereas the burst duration becomes shorter during prolonged culture of the iCell $^{\circ}$ Glutaneurons - iCell ${ }^{\circ}$ Astrocytes co-culture (Fig. 3A), it slightly increases for the other two models (Fig. 3B-C). For the iCell ${ }^{\circ}$ Glutaneurons - iCell ${ }^{\circ}$ Astrocytes co-culture (Fig. 3A) and the CNS. $4 \mathrm{U}^{\circ}$ co-culture (Fig. 3B) the number of spikes per network burst and network burst percentage increase over time, in contrast to the SynFire iNs co-culture (Fig. $3 \mathrm{C}$ ) where this pattern is relatively stable. The IBI is longest in early culture DIVs for the iCell $^{\circ}$ Glutaneurons - iCell $^{\circ}$ 
Astrocytes co-culture and the SynFire ${ }^{\circ}$ iNs co-culture, whereas for the CNS. $4 \mathrm{U}^{\circ}$ co-culture the IBI is relatively stable during the entire culture period. The number of spikes per burst increases during the culture period resulting in an increasing burst percentage for the iCell ${ }^{\circ}$ Glutaneurons - iCell $^{\circ}$ Astrocytes co-culture and the SynFire iNs, whereas this is relatively stable for the CNS. $4 \mathrm{U}^{\circ}$ co-culture.
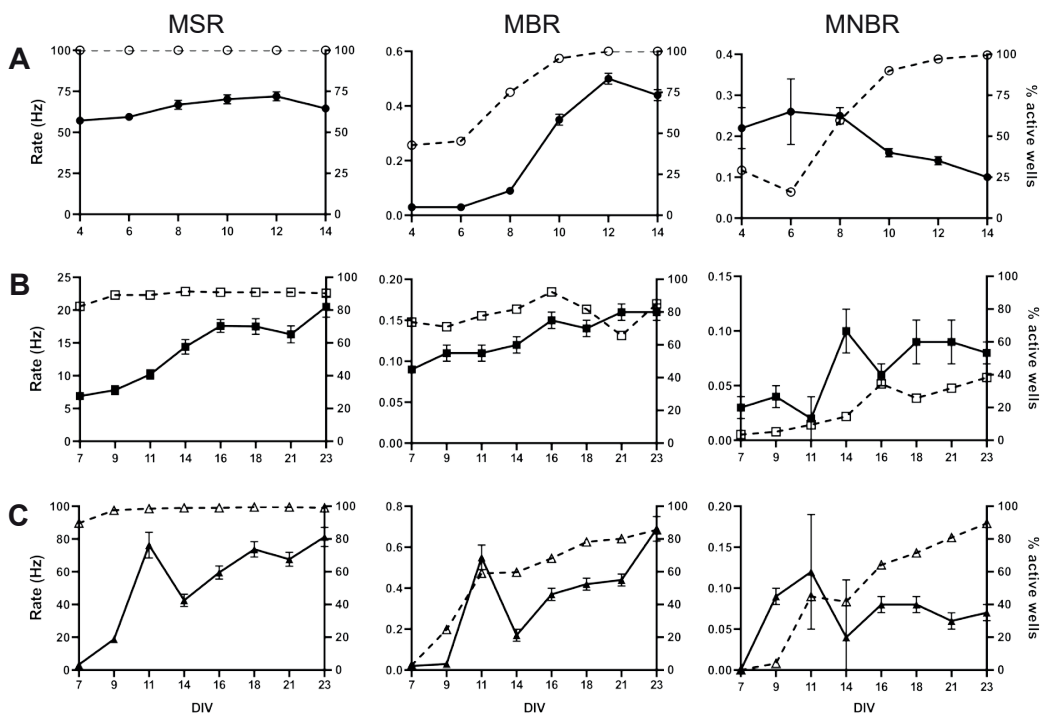

Figure 2. Development of mean spike rate (MSR; left), mean burst rate (MBR; middle) and mean network burst rate (MNBR: right) in solid lines and percentage of spiking wells (left), bursting wells (middle) and network bursting wells (right) in dashed lines for iCell ${ }^{\circ}$ Glutaneurons - iCell ${ }^{\circ}$ Astrocytes co-culture (A), CNS. $4 \mathrm{U}^{\circ}$ co-culture (B) and SynFire iNs co-culture (C). Data are expressed as mean \pm SEM from $n=26-208$ wells, $N=5$ plates for the iCell ${ }^{\circ}$ Glutaneurons - iCell $^{\circ}$ Astrocytes co-culture; $\mathrm{n}=4-170$ wells, $\mathrm{N}=5$ plates for the $\mathrm{CNS} .4 \mathrm{U}^{\circ}$ co-culture and $\mathrm{n}=2-202$ wells, $\mathrm{N}=8$ plates for the SynFire ins co-culture.

The pattern of spontaneous electrical activity at the exposure DIV, the day in vitro at which the chemical sensitivity of the compound was assessed, is depicted in spike raster plots (Fig. 4) and further described by different metric parameters (Table 3). The spike raster plots show that all three models exhibit a mature phenotype with synchronised firing with (network) bursts. The raster plots also confirm that the pattern and level of activity differs between the models. The higher MSR for the iCell ${ }^{\circledR}$ Glutaneurons - iCell ${ }^{\circledR}$ Astrocytes co-culture (Fig. 4A) 
A

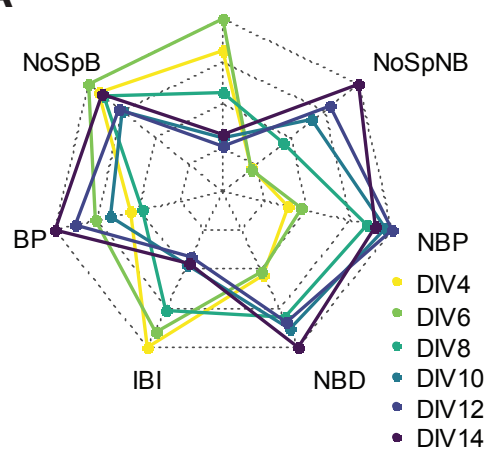

B

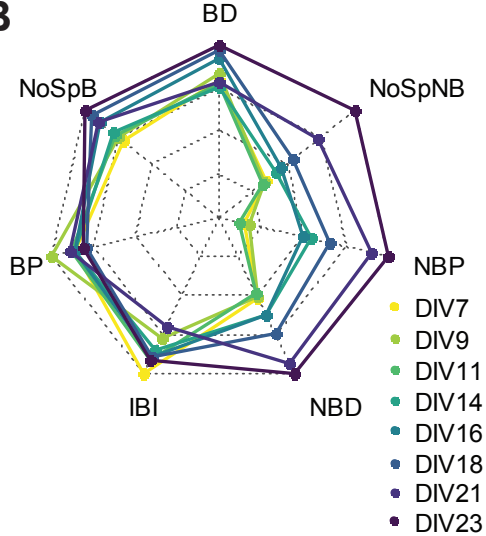

C

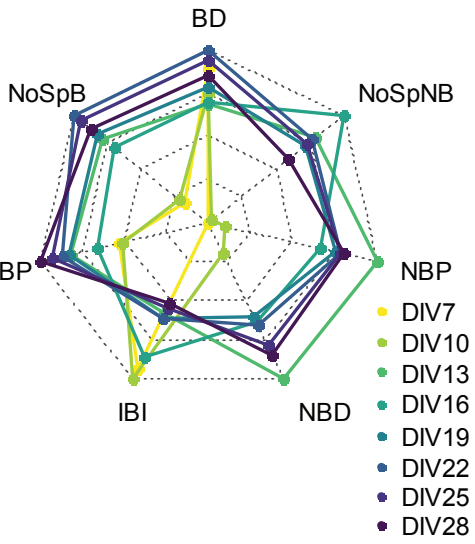

Figure 3. Spider plots illustrating the differences in development of neuronal activity between the iCell $^{\circ}$ Glutaneurons - iCell $^{\circ}$ Astrocytes co-culture (A), CNS. $4 \mathrm{U}^{\circ}$ co-culture (B) and SynFire iNs co-culture (C). The yellow and lighter green lines depict earlier developmental DIVs, whereas darker shades belong to DIVs later in development. The grey grid represents $0 \%$ at the centre, whereas the outer ring represents $100 \%$. For each model, the highest value of a parameter during the culture period of that model is set to $100 \%$. The axis represent (counter clockwise): burst duration (BD), number of spikes per burst (NoSpB), burst percentage (BP), inter-burst interval (IBI), network burst duration (NBD), network burst percentage (NBP) and number of spikes per network burst (NoSpNB), from $n=15-208$ wells, $N=$ 5 plates for the iCell ${ }^{\circ}$ Glutaneurons - iCell $^{\circ}$ Astrocytes co-culture; $n=4-167$ wells, $N=5$ plates for the $\mathrm{CNS} .4 \mathrm{U}^{\circ}$ co-culture; $\mathrm{n}=2-202$ wells, $\mathrm{N}=8$ plates for the SynFire iNs coculture. 
Table 3. Mean \pm SEM of different metric parameters at the exposure DIV.

\begin{tabular}{|c|c|c|c|c|}
\hline Metric parameter (unit) & $\begin{array}{l}\text { iCell }^{\circledR} \\
\text { Glutaneurons - } \\
\text { iCell }^{\circledR} \text { Astrocytes } \\
\text { (DIV14) }\end{array}$ & $\begin{array}{l}\text { CNS.4U } \\
\text { (DIV23) }\end{array}$ & $\begin{array}{l}\text { SynFire }^{\circledR} \text { iNs } \\
\text { (DIV28) }\end{array}$ & $\begin{array}{l}\text { Rat cortical } \\
\text { neurons } \\
\text { (DIV11) }\end{array}$ \\
\hline $\operatorname{MSR}(\mathrm{Hz})$ & $64.5 \pm 2.39$ & $20.5 \pm 1.60$ & $81.3 \pm 5.86$ & $21.4 \pm 0.82$ \\
\hline ISI coefficient of variation & $1.86 \pm 0.029$ & $1.93 \pm 0.046$ & $3.36 \pm 0.15$ & $4.74 \pm 0.10$ \\
\hline Number of active electrodes & $14.4 \pm 0.13$ & $8.68 \pm 0.29$ & $13.3 \pm 0.27$ & $13.4 \pm 0.23$ \\
\hline Number of active wells & 208 & 166 & 201 & 236 \\
\hline $\mathrm{MBR}(\mathrm{Hz})$ & $0.44 \pm 0.017$ & $0.16 \pm 0.014$ & $0.69 \pm 0.057$ & $0.77 \pm 0.028$ \\
\hline Number of bursting electrodes & $9.78 \pm 0.28$ & $3.96 \pm 0.28$ & $11.5 \pm 0.43$ & $13.2 \pm 0.23$ \\
\hline Number of bursting wells & 208 & 141 & 172 & 234 \\
\hline Burst duration (s) & $0.98 \pm 0.036$ & $1.15 \pm 0.06$ & $0.97 \pm 0.033$ & $0.32 \pm 0.012$ \\
\hline Number of spikes per burst & $38.9 \pm 1.35$ & $28.7 \pm 1.07$ & $73.3 \pm 3.50$ & $22.7 \pm 0.55$ \\
\hline Mean ISI within burst (s) & $0.03 \pm 0.0007$ & $0.045 \pm 0.002$ & $0.023 \pm 0.001$ & $0.02 \pm 0.001$ \\
\hline IBI (s) & $33.8 \pm 0.97$ & $44.0 \pm 2.22$ & $37.66 \pm 2.47$ & $25.5 \pm 0.94$ \\
\hline IBI coefficient of variation & $0.69 \pm 0.019$ & $0.80 \pm 0.031$ & $0.80 \pm 0.046$ & $0.83 \pm 0.037$ \\
\hline Burst percentage (\%) & $34.8 \pm 0.76$ & $34.7 \pm 1.62$ & $52.3 \pm 1.84$ & $83.3 \pm 0.52$ \\
\hline $\operatorname{MNBR}(\mathrm{Hz})$ & $0.1 \pm 0.006$ & $0.08 \pm 0.012$ & $0.074 \pm 0.005$ & $0.073 \pm 0.002$ \\
\hline Network burst duration (s) & $0.92 \pm 0.088$ & $0.88 \pm 0.076$ & $2.12 \pm 0.18$ & $0.75 \pm 0.015$ \\
\hline Number of spikes per network burst & $306 \pm 20.1$ & $166 \pm 22.6$ & $1350 \pm 106$ & $285 \pm 7.83$ \\
\hline Mean ISI within network burst (s) & $0.004 \pm 0.0002$ & $\begin{array}{l}0.007 \pm \\
0.0004\end{array}$ & $0.003 \pm 0.0002$ & $0.004 \pm 0.00$ \\
\hline Number of electrodes network burst & $13.2 \pm 0.14$ & $10.1 \pm 0.36$ & $14.4 \pm 0.20$ & $13.6 \pm 0.15$ \\
\hline Number of network bursting wells & 206 & 54 & 154 & 225 \\
\hline Network burst percentage (\%) & $37.1 \pm 1.32$ & $29.8 \pm 2.87$ & $64.7 \pm 2.53$ & $93.0 \pm 0.62$ \\
\hline Network IBI coefficient of variation & $0.73 \pm 0.023$ & $0.56 \pm 0.039$ & $0.83 \pm 0.064$ & $0.78 \pm 0.035$ \\
\hline Network normalised duration IQR & $0.51 \pm 0.043$ & $0.51 \pm 0.091$ & $0.56 \pm 0.13$ & $0.71 \pm 0.030$ \\
\hline $\begin{array}{l}\text { Area under normalised cross- } \\
\text { correlation }\end{array}$ & $0.11 \pm 0.003$ & $0.016 \pm 0.002$ & $0.25 \pm 0.012$ & $0.56 \pm 0.011$ \\
\hline $\begin{array}{l}\text { FWHH of normalised cross- } \\
\text { correlation }\end{array}$ & $128 \pm 1.60$ & $210 \pm 18.0$ & $734 \pm 21.3$ & $22.8 \pm 0.36$ \\
\hline
\end{tabular}


and SynFire ${ }^{\circledR}$ iNs co-culture (Fig. 4 C), as described in Table 3 and Fig. 2, is also visible in the raster plots. The spike time histogram (green box, Fig. 4) clearly shows the different levels of synchronicity that are outlined in Table 3 , and indicate the high number of spikes within a network burst in the SynFire ${ }^{\circledR}$ iNs co-culture. Further details regarding the other developmental days of the hiPSC-derived cocultures can be found in the supplemental material (Table S1-S3 and Fig. S1).

As can be seen in the raster plots, rat primary cortical neurons exhibit a spike frequency comparable to the CNS.4U ${ }^{\circledR}$ co-culture (Fig. 4D, Table 3), whereas the burst frequency is more in line with the SynFire ${ }^{\circledR}$ iNs co-culture. Network burst activity of rat primary cortical neurons occurs in a frequency comparable to the CNS.4U ${ }^{\circledR}$ co-culture and the SynFire ${ }^{\circledR}$ iNs co-culture. The burst duration of the primary rodent culture is much lower than in the hiPSC-derived co-cultures. In rat primary cortical neurons most spikes are incorporated in (network) bursts as is reflected in the (network) burst percentage. This is in sharp contrast with hiPSC-derived neuronal models, where single spikes occur.

\subsection{Seizure liability assessment}

Since all models developed spontaneous neuronal activity and (network) bursting behaviour, all hiPSC-derived neuronal models are amenable for neurotoxicity testing. To assess their applicability for seizure liability assessment, the different models were exposed to known seizurogenic compounds with different modes of action: strychnine $(0.3-30 \mu \mathrm{M})$, 4-AP (1-100 $\mu \mathrm{M})$ and PTX $(0.1-10 \mu \mathrm{M})$. We also exposed rat primary cortical neurons as the current gold standard for MEA seizure liability assessment.

Exposure to the known neurotoxicant strychnine, a glycine receptor antagonist, results in an increase of the MSR followed by a decrease at higher test concentrations in the SynFire iNs co-culture and the rat primary cortical culture (Fig. 5A; left). However, compared to the rat primary cortical neurons, the increase starts in the SynFire iNs co-culture at a concentration 3 times lower. Strychnine significantly decreases the MSR of the $\mathrm{iCell}^{\circ}$ Glutaneurons - $\mathrm{iCell}^{\circ}$ Astrocytes coculture and the CNS. $4 \mathrm{U}^{\circ}$ co-culture. MBR increases in the CNS. $4 \mathrm{U}^{\circ}$ co-culture, SynFire ${ }^{\circ}$ iNs co-culture and rat primary cortical culture (Fig 5B; left). However, this increase is only significant in the latter. At the highest concentration tested, the MBR significantly decreases in the $\mathrm{iCell}^{\circ}$ Glutaneurons - iCell ${ }^{\circ}$ Astrocytes coculture and the CNS. $4 \mathrm{U}^{\circ}$ co-culture. Strychnine exposure significantly increases the MNBR in the rat primary cortical culture (Fig. 5C; left). However, this cannot be observed in the hiPSC-derived neuronal models. Burst duration increases in 
A

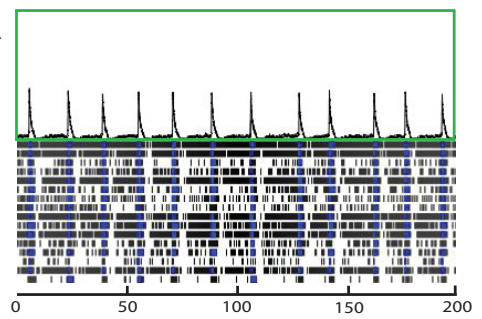

C

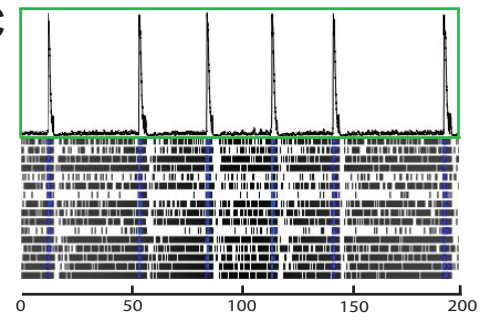

B
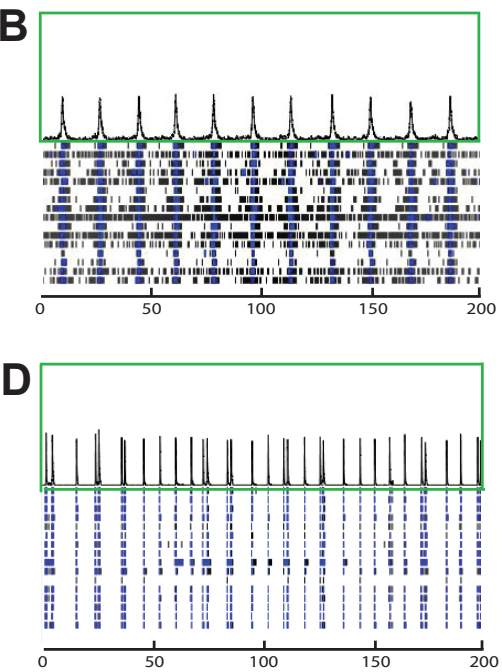

Figure 4. Spike raster plots of the different hiPSC-derived co-cultures. Each row depicts one electrode, each tick mark represents one spike in a $200 \mathrm{~s}$ interval illustrating the pattern of

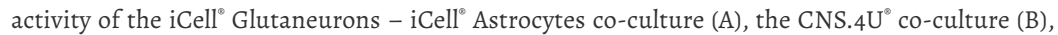
the SynFire iNs co-culture (C) and the primary rat cortical neurons (D) at the exposure DIV. The cumulative trace (green box) above each plot depicts the population spike time histogram, indicating synchronized activity between the different electrodes.

the CNS. $4 \mathrm{U}^{\circledast}$ co-culture and the iCell ${ }^{\oplus}$ Glutaneurons - $\mathrm{iCell}^{\circledR}$ Astrocytes co-culture, but only significantly in the latter (Fig. 5D; left). A significant decrease in network burst duration following strychnine exposure can be observed in rat primary cortical neurons (Fig. 5E; left).

PTX, a widely used seizurogenic reference compound and $\mathrm{GABA}_{\mathrm{A}}$-receptor antagonist, shows limited effects on the MSR of $\mathrm{iCell}^{\circ}$ Glutaneurons - $\mathrm{iCell}^{\circ}$ Astrocytes co-culture and CNS. $4 \mathrm{U}^{\circ}$ co-culture (Fig. 5A; middle). However, PTX significantly increases the MSR of the SynFire iNs co-culture and the rat primary cortical culture at the higher concentrations tested. Higher concentrations of PTX significantly increase the MBR of the SynFire iNs co-culture and the rat primary cortical culture, but have little effect on $\mathrm{iCell}^{\circ}$ Glutaneurons - iCell ${ }^{\circ}$ Astrocytes co-culture and the CNS. $4 \mathrm{U}^{\circ}$ co-culture (Fig. 5B; middle). In all cultures, PTX increases the MNBR (Fig. 5C; middle), but only significantly in the rat primary cortical culture. A significant increase of burst duration can be observed for the 
iCell $^{\circ}$ Glutaneurons - iCell $^{\circ}$ Astrocytes co-culture, whereas a decrease occurs in the rat primary cortical culture (Fig. 5D; middle). Network burst duration significantly increases for the iCell ${ }^{\circ}$ Glutaneurons - iCell $^{\circ}$ Astrocytes co-culture and the SynFire iNs co-culture (Fig. 5E; middle). However, network burst duration decreases in the rat primary cortical culture.

4-AP, a known CNS stimulant and potassium channel blocker, only increases the MSR of the SynFire iNs co-culture (Fig. 5A; right). MBR increases when the iCell $^{\circ}$ Glutaneurons - iCell ${ }^{\circ}$ Astrocytes co-culture and SynFire iNs co-culture are exposed, although not significantly (Fig. 5B; right). Exposure to 4-AP significantly decreases the MBR of the CNS. $4 \mathrm{U}^{\circ}$ co-culture. This is in contrast with the increased MNBR following 4-AP exposure of the $\mathrm{CNS} .4 \mathrm{U}^{\circ}$ co-culture (Fig. $5 \mathrm{C}$; right). An increase can also be observed in the SynFire iNs co-culture, whereas the iCell ${ }^{\circ}$ Glutaneurons - iCell ${ }^{\circ}$ Astrocytes co-culture and rat primary cortical culture are relatively unaffected. Following exposure to 4-AP, burst duration significantly decreases in all hiPSC-derived co-cultures, but this decrease occurs at different concentrations (Fig. 5D; right). Network burst duration increases in the SynFire ${ }^{\circ}$ iNs co-culture, but decreases in the $\mathrm{CNS}_{4} 4 \mathrm{U}^{\circ}$ co-culture and rat primary cortical culture (Fig. $5 \mathrm{E}$; right).

The activity patterns of the different models change following exposure. More details can be found in the supplemental data (Figure S2 for strychnine, S3 for PTX and S4 for 4-AP).

We created a heatmap of the concentration-response curves of the three test compounds on the four different models to further illustrate the effects on different metric parameters (Fig. 6). By the different colour pattern in the heatmap, it becomes clear that strychnine, PTX and 4-AP have different mechanisms of action. For all compounds, but particularly for PTX, it is visible that network burst related parameters are most affected when it comes to excitation. Burst related parameters are more sensitive to inhibition, especially following 4-AP or strychnine exposure.

Several similarities and differences between the models can be observed. Following exposure to strychnine, seizurogenicity is most noticeable in the network burst related parameters in the SynFire ${ }^{\circ}$ iNs co-culture. Inhibition following strychnine exposure is most pronounced in the CNS. $4 \mathrm{u}^{\circ}$ co-culture. 
When the models are exposed to PTX, all models show a clear excitation. The iCell ${ }^{\circ}$ Glutaneurons - iCell ${ }^{\circ}$ Astrocytes co-culture and the CNS. $4 \mathrm{U}^{\circ}$ co-culture react in a comparable pattern, whereas the SynFire iNs co-culture shows more similarities with the rat primary cortical culture.

The only model that is excitable with 4-AP on all three traditional parameters (MSR, MBR and MNBR) is the SynFire iNs co-culture. The iCell ${ }^{\circ}$ Glutaneurons - iCell $^{\circ}$ Astrocytes co-culture shows excitability on burst and network burst parameters at concentrations lower than the rat primary cortical culture. Overall, the SynFire iNs co-culture is most easily excited, whereas the $\mathrm{CNS}^{\circ} 4 \mathrm{U}^{\circ}$ co-culture most easily inhibited. 

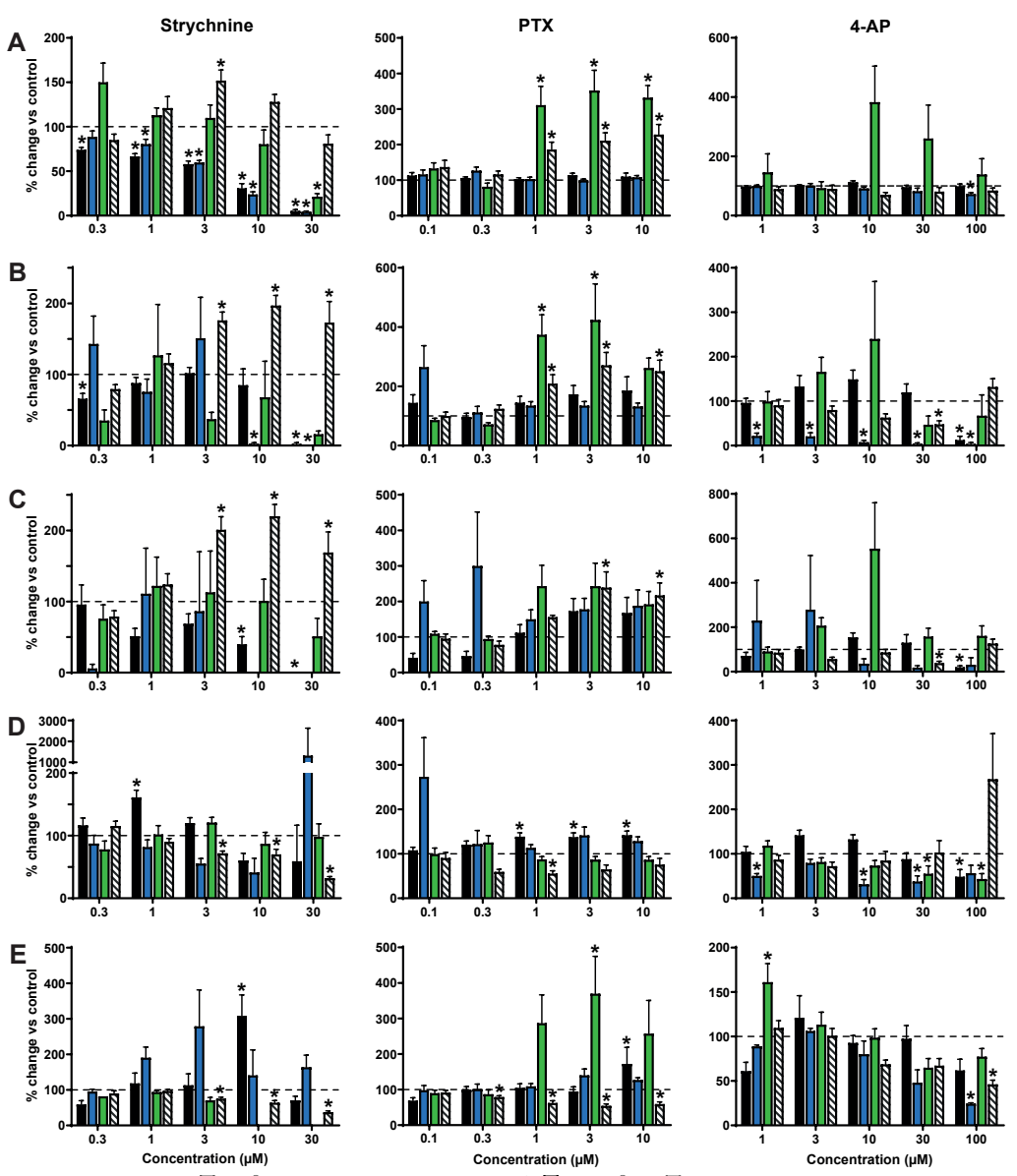

Figure 5. Toxicological modulation of spontaneous network activity with strychnine (left), PTX (middle) and 4-AP (right) of iCell ${ }^{\circ}$ Glutaneurons - iCell ${ }^{\circ}$ Astrocytes co-culture (black), CNS.4U ${ }^{\circ}$ co-culture (blue), SynFire iNs co-culture (green) and rat cortical neurons (striped). Effects are depicted on mean spike rate(MSR;A), mean burst rate (MBR; B), mean network burst rate (MNBR; C), burst duration (D) and network burst duration (E) as average in \% change of control (solvent control set to $100 \%$; dashed line) \pm SEM from $n=3-17$ wells, $N=2-3$ plates for iCell ${ }^{\circ}$ Glutaneurons - iCell ${ }^{\circ}$ Astrocytes co-culture; $\mathrm{n}=2-16$ wells, $\mathrm{N}=4$ plates for $\mathrm{CNS} .4 \mathrm{U}^{\circ}$ co-culture; $\mathrm{n}=3-14$ wells, $\mathrm{N}=4-5$ plates for SynFire iNs co-culture and $\mathrm{n}=19-29, \mathrm{~N}=5-8$ for rat primary cortical culture. $" p<0.05$. 


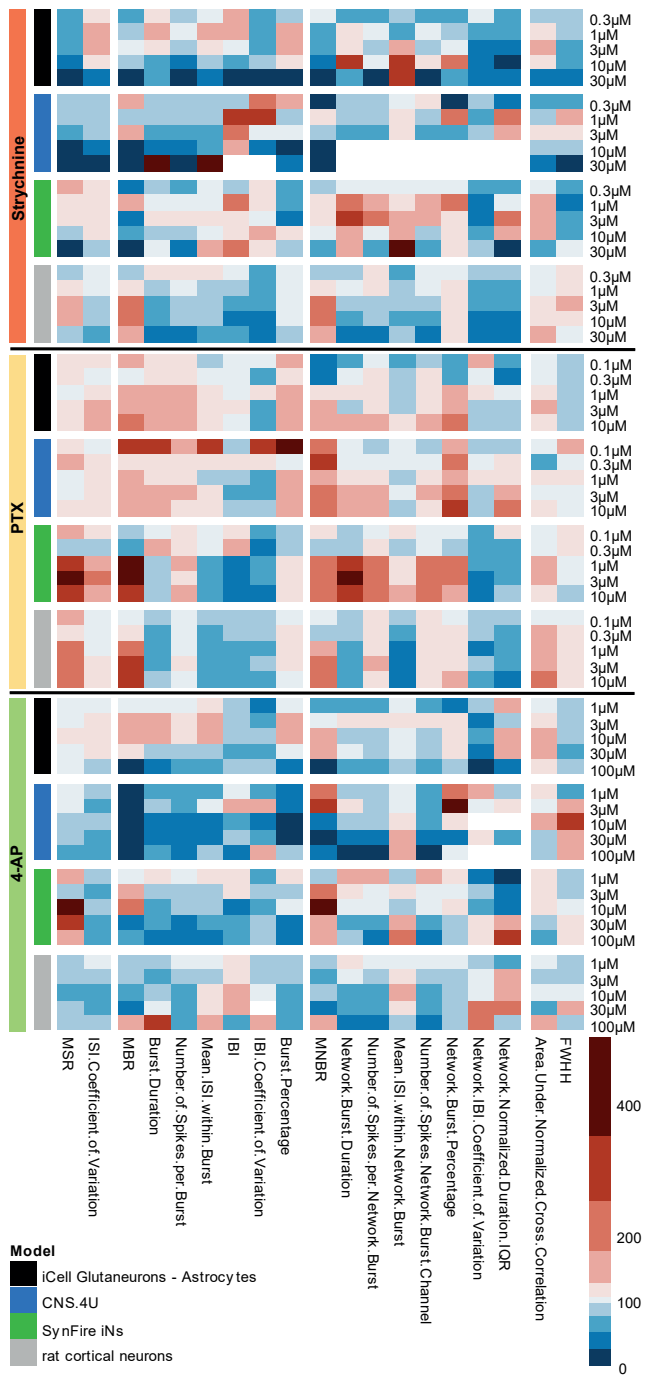

Figure 6. Heatmap of the effects of strychnine (0.3-30 $\mu \mathrm{M}$; top), PTX (0.1 - $10 \mu \mathrm{M}$; middle) and 4-AP (1 - $100 \mu \mathrm{M}$; bottom) on selected metric parameters on iCell $^{\circ}$ Glutaneurons iCell $^{\circ}$ Astrocytes co-culture (black), CNS. $4 \mathrm{U}^{\circ}$ co-culture (blue) SynFire iNs co-culture (green) and rat primary cortical neurons (grey). Colour scaling is based on the magnitude of the $\%$ of change relative to the control based on $\mathrm{n}=$ 1-18 wells, $N=2-3$ plates for iCell $^{\circ}$ Glutaneurons - iCell $^{\circ}$ Astrocytes co-culture; $\mathrm{n}=1-16$ wells, $\mathrm{N}=4$ plates for $\mathrm{CNS} .4 \mathrm{U}^{\circ}$ co-culture; $\mathrm{n}=2-15$ wells, $\mathrm{N}$ $=4-5$ plates for SynFire iNs co-culture and $\mathrm{n}=11-30$ wells, $\mathrm{N}=5-8$ plates for rat primary cortical culture. For white compartments no average could be calculated. 


\section{DISCUSSION}

In this study we investigated the applicability of three different human iPSCderived neuronal models for more predictive in vitro seizure liability assessment without the use of animals.

Our immunocytochemistry data (Fig. 1) demonstrated the mixed nature of the different hiPSC-derived neuronal models. The images show that all models form highly complex neuronal networks comparable with the ones formed by rat primary cortical cultures (Hondebrink et al., 2016). All three models develop spontaneous neuronal network activity and (network) bursting behaviour over time (Fig. 2-4). However, the level of activity and bursting varies between the different models, despite culturing according to manufacturer's protocol. There are several possible explanations for these differences. One likely explanation relates to cell density as the amount of cells plated in mwMEAs has been shown to greatly influence the spontaneous firing rate, with higher densities resulting in a higher spontaneous activity rate (Jun et al., 2007). In line with this notion, the model with the lowest seeding density ( $\mathrm{CNS} .4 \mathrm{U}^{\circ}$ co-culture; Table 1) also has the lowest spike and burst activity (Fig. 2). The cell density of the $\mathrm{CNS} .4 \mathrm{U}^{\circ}$ co-culture is also low compared to densities reported in literature (Odawara et al., 2016; Matsuda et al., 2018; Sasaki et al., 2019). Cell densities of the iCell ${ }^{\circ}$ Glutaneurons - iCell ${ }^{\circ}$ Astrocytes co-culture and in particular of the SynFire iNs co-culture are more in line with literature and this is paralleled by a higher spontaneous activity level. Spontaneous firing rate is also enhanced by the presence of astrocytes (Tang et al., 2013; Ishii et al., 2017; Tukker et al., 2018). The higher ratio of astrocytes in the $\mathrm{iCell}^{\circ} \mathrm{Glutaneurons}-\mathrm{iCell}^{\circ}$ Astrocytes co-culture and SynFire iNs co-culture (Table 1) could contribute to their higher spike and burst rate (Fig $2 \mathrm{~A}-\mathrm{B}$ ). The SynFire iNs co-culture is the culture with the highest ratio of astrocytes, which coincides with the highest degree of synchronisation (Fig. 4). Additionally, human iPSC-derived neuronal cultures with a high ratio of glutamatergic neurons exhibit more synchronous bursting events compared to cultures with a low proportion glutamatergic neurons (Sasaki et al., 2019). At the first culture days, the iCell ${ }^{\circ}$ Glutaneurons - iCell $^{\circ}$ Astrocytes co-culture, the culture with the highest number of glutamatergic neurons, exhibited the highest MNBR (Fig. 2C). However, at the day of exposure, MNBR was comparable for all cultures. Despite their differences, all the models tested, exhibited a pattern of development comparable to that of the rat primary cortical culture (Brown et al., 2016) with MSR, presence of (network) bursts and synchronised activity increasing over time. 
Since all models develop spontaneous activity, a neurotoxicity screen was performed using the known seizurogenic compounds strychnine, PTX and 4-AP. These compounds are used in in vivo studies to induce seizures (Peña and Tapia, 2000; Mackenzie et al., 2002; Alachkar et al., 2018). Strychnine and 4-AP have also been reported to cause seizures in humans (Pickett and Enns, 1996; van Berlo-van de Laar et al., 2015). Overall, our data indicate the potential of the hiPSC-derived neuronal models for in vitro seizure liability assessment as all three compounds increased activity and synchronicity of the networks.

The potential for seizure liability assessment is further confirmed when comparing our data with two seizure prediction patterns described in literature (Bradley et al., 2018). Seizure prediction pattern 1 is indicative for the response induced by $\mathrm{GABA}_{\mathrm{A}}$-receptor antagonists, such as PTX. This pattern is characterised by an overall increase in activity as well as an increased organisation and synchronisation, reflected in an increased number of spikes per burst, increased ISI coefficient of variation, increased burst duration and a decrease in IBI. All four tested models showed an increase in overall activity following exposure to PTX (Fig. 5). Spiking activity increased in a concentration-dependent manner only in the SynFire iNs co-culture and primary rat cortical neurons similar to results reported in literature for rat primary cortical cultures (Mack et al., 2014; Kreir et al., 2018) and hiPSC-derived neuronal co-cultures (Kreir et al., 2019). A rise in activity was observed for all models in (network) bursting and comparable to results from Kreir et al. (2019). Also, the organisation and synchronisation are enhanced according to prediction pattern 1 (Fig. 6).

Seizure prediction pattern 2 is characterised by a decrease in overall activity and a deterioration of network organisation, reflected in an increased IBI, a decreased number of spikes per burst and a decreased ISI coefficient of variation and burst duration. This pattern is indicative for exposure to the glycine receptor antagonist strychnine (Bradley et al., 2018). The iCell ${ }^{\bullet}$ Glutaneurons - iCell $^{\oplus}$ Astrocytes co-culture and $\mathrm{CNS}_{4} 4 \mathrm{U}^{\circ}$ co-culture exhibited decreased activity (Fig. 5) and network disintegration (Fig. 6) following the described prediction pattern. Changes in spike and burst activity at the lowest test concentration in the iCell ${ }^{\circledR}$ Glutaneurons - iCell ${ }^{\circledR}$ Astrocytes co-culture are statistically significant, indicating a high sensitivity of this model for the tested glycine receptor agonist. However, this does not necessarily indicate biological relevance. In contrast to earlier reported findings (Bradley et al., 2018; Kreir et al., 2018), activity of the rat primary cortical culture increased following strychnine exposure. However, 
this can be due to the differences in maturation stage between E18-19 and PND1 cortices. During embryonic development, glycine receptors have an excitatory function, whereas they become inhibitory later on (Dutertre et al., 2012).

Exposure to the potassium channel blocker 4-AP is reported to follow seizure prediction pattern 1 (Bradley et al., 2018), however our data do not support that notion (Fig. 5-6). An increase in overall activity is only observed in the SynFire iNs co-culture and an increase in bursting activity in the iCell $^{\circ}$ Glutaneurons - iCell $^{\circ}$ Astrocytes co-culture (Fig. 5), but the increased organisation and synchronisation to match pattern 1 is not present (Fig. 6). Kreir et al. (2019) reported an increase in spike and network burst frequency following exposure of hiPSC-derived neuronal cultures to strychnine. This matches with our SynFire iNS co-culture data. The other cultures showed a decreased activity pattern, but with a network deterioration that did not match prediction pattern 2. Rat hippocampal neurons cultured on MEA plates exhibit increased activity (Fan et al., 2019). It could thus be that a more hippocampal-like phenotype is required to detect the seizurogenic activity of 4-AP.

When comparing the differences in sensitivity of the models, the aforementioned points in regards to ratio of excitatory neurons to inhibitory neurons, number of astrocytes present and seeding density must be kept in mind, since these can all affect chemical sensitivity. Although cells were exposed at a moment of network maturation and according to continuously improving manufacturer's protocol, network complexity (e.g. the number of contacts that the different cell types establish) can still be different for the different models. This may influence how the models react to chemical insults. Also, receptor expression may differ between the different hiPSC-derived neuronal models. Spontaneous activity and drug responses are dictated by a complex interplay of a large number of different receptor types, ion channels and transporters all consisting of different subtypes. To better understand the differences in chemical sensitivity between the models, full protein profiles of the expression levels of the different receptors, channels and transporters present must be made. However, not only the number of receptors, channels and transporters are crucial, also the interplay, location and distribution of the receptors, channels and transporters define chemical sensitivity.

We showed that all tested models were able to form spontaneously active networks with (network) bursting behaviour. Our data also indicated that these models can be used for animal-free in vitro seizure liability assessment. More importantly, the 
iPSC-derived models were capable of detecting seizures at the same level or even better than the rat primary cortical neuronal culture. However, in order to detect seizures in vitro with an MEA system, multiple parameters must be assessed. When only spike behaviour is investigated, seizure-like activity might be missed. Also, when comparing data from hiPSC-derived neuronal models with rat primary cortical cultures, it must be kept in mind that there are differences between rodents and humans. Hence, rodent experiments are not always predictive for human risk (Olson et al., 2000). It is thus logical that results differ between in vitro assays that are performed using human cells and assays using rodent cells (Hondebrink et al., 2017). This in turn results in different hits and sensitivities (Malik et al., 2014). However, there are also cases where the rodent and human system show comparable results (Kasteel and Westerink, 2017). The discrepancies between the rat primary rodent cortical culture and our hiPSC-derived neuronal models should not be the main concern. Rather, attention must be on further characterisation and optimisation of hiPSC-derived neuronal models to make them more closely resemble the human brain, specifically those parts that are involved in epileptic activity such as the amygdala, hippocampus and neocortex (Rogawski and Löscher, 2004). Also, it must be kept in mind that this study was done with a limited number of test compounds. To fully understand the applicability of hiPSC-derived neuronal models for in vitro seizure liability assessment, a broader set of compounds should be tested, including besides seizurogenics, anti-seizurogenic and negative compounds.

Nevertheless, the current data show that hiPSC-derived neuronal models may already be used as a first screen for epileptic activity before performing follow-up studies. With that we are one step closer to animal-free in vitro seizure liability assessment. Nevertheless, to fully move away from animal testing for in vitro seizure liability assessment, hiPSC-models must be developed in which all targets involved in the onset and duration of seizures are present and validated using a large library of diverse reference compounds. With respect to the comparison of hiPSC-derived neuronal cultures with rodent primary neuronal cultures; such a comparison cannot be performed one on one. Actually, our data show some striking differences between the different models, highlighting the need to move to human model systems, thereby eliminating the need for interspecies extrapolation. 


\section{CONFLICT OF INTERREST}

The authors declare that they have no conflict of interest.

\section{ACKNOWLEDGEMENTS}

We gratefully acknowledge members of the Neurotoxicology Research Group for helpful discussions. We thank Richard Wubbolts and Esther van 't Veld (Center for Cell Imaging, Utrecht University) for help with the microscopy work and Barbara Vreede (University library, Utrecht University) for assisting with $\mathrm{R}$ programming of the heatmap. This work was funded by a grant from the National Centre for the Replacement, Refinement and Reduction of Animals in Research (NC3Rs; project number 50308-372160) and by the Faculty of Veterinary Medicine (Utrecht University, The Netherlands). 


\section{SUPPLEMENTAL DATA}
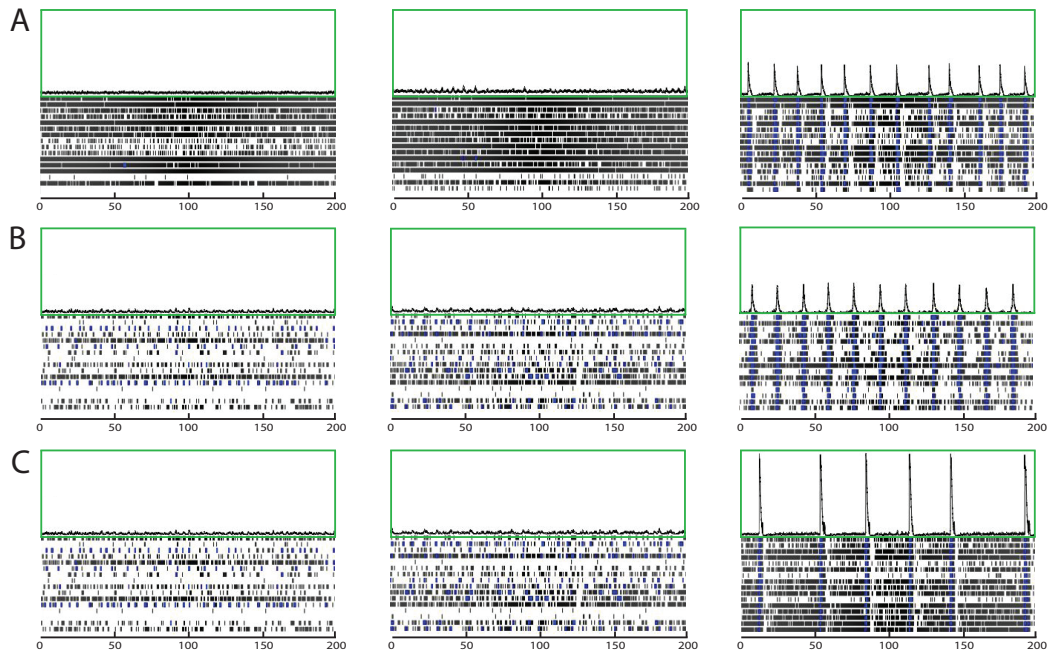

Figure S1. Spike raster plots of the different hiPSC-derived co-cultures. Each row depicts one electrode, each tick mark represents one spike in a $200 \mathrm{~s}$ interval illustrating the pattern of activity of the iCell ${ }^{\circ}$ Glutaneurons - iCell ${ }^{\circ}$ Astrocytes co-culture (A) at DIV4 (left), DIV8 (middle) and DIV14 (right); the CNS.4U $\mathrm{U}^{\circ}$ co-culture (B) at DIV7 (left), DIV16 (middle) and DIV23 (right) and the SynFire iNs co-culture (C) at DIV7 (left), DIV19 (middle) and DIV28 (right). The cumulative trace (green box) above each plot depicts the population spike time histogram, indicating synchronized activity between the different electrodes. 


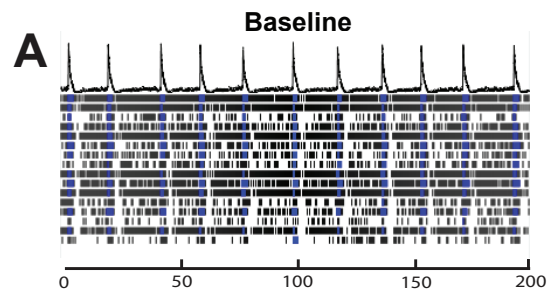

\section{Exposure $3 \mu \mathrm{M}$}

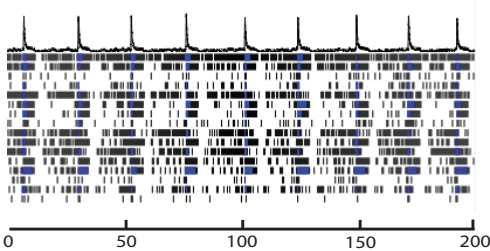

B
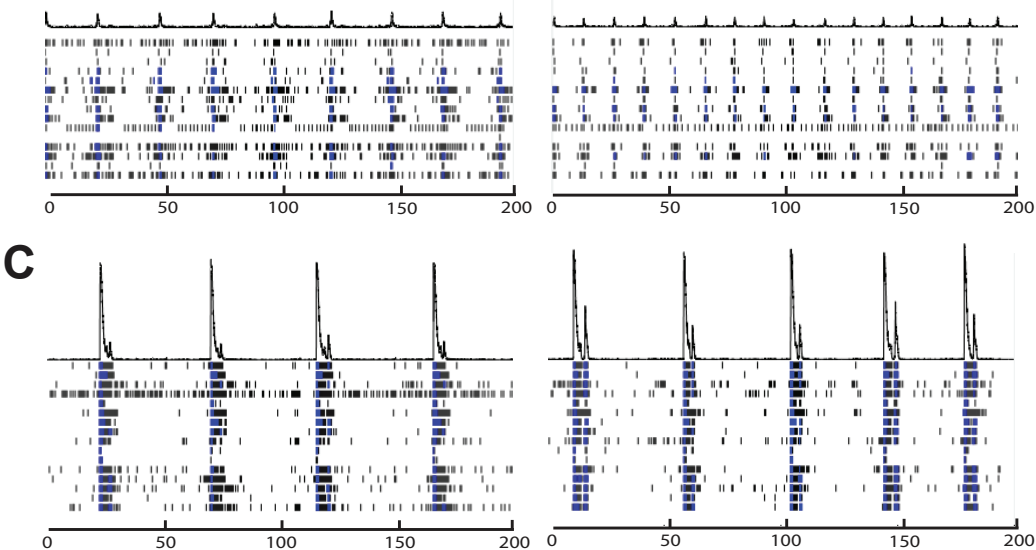

D
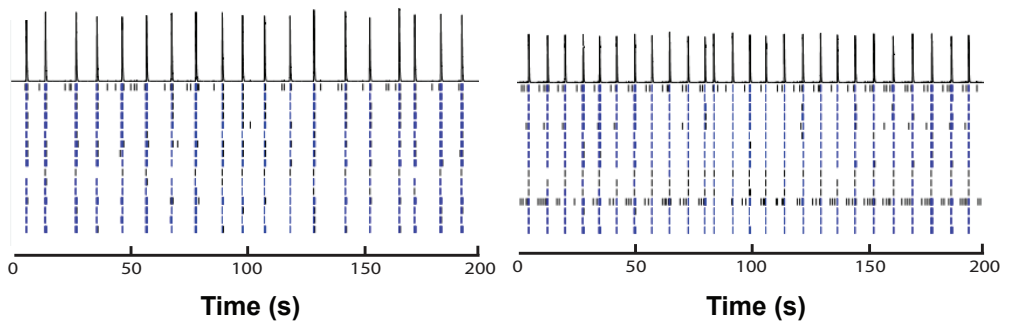

Figure S2. Spike raster plots of Cell ${ }^{\circ}$ Glutaneurons - iCell ${ }^{\circ}$ Astrocytes co-culture (A), CNS. $4 \mathrm{U}^{\circ}$ coculture (B), SynFire iNs co-culture (C) and rat primary cortical neurons (D) before (left) and after exposure to $3 \mu \mathrm{M}$ strychnine (right). Each row depicts one electrode, each tick mark represents one spike in a $200 \mathrm{~s}$ interval illustrating the pattern of activity. The cumulative trace above each plot depicts the population spike time histogram, indicating the level of synchronized activity between the different electrodes. 

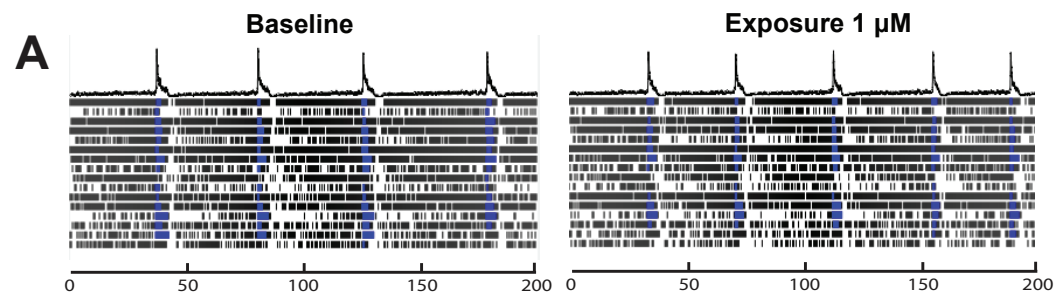

B
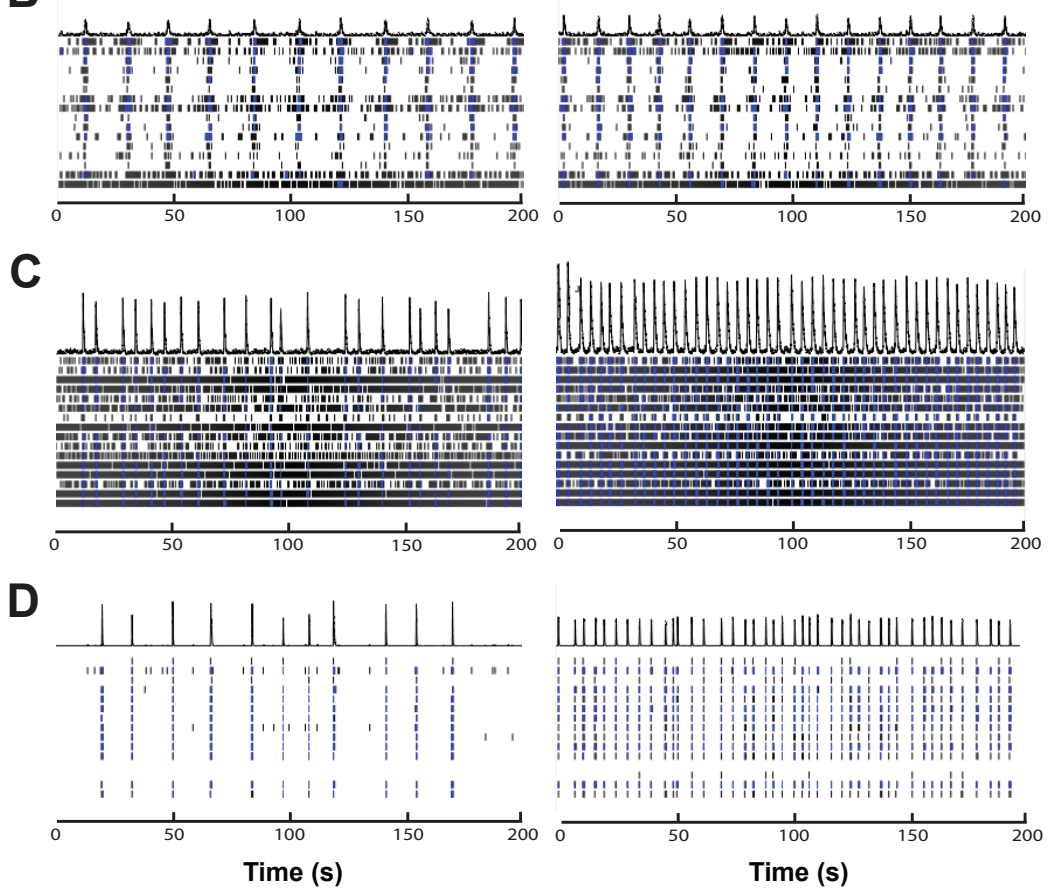

Figure S3. Spike raster plots of Cell ${ }^{\circ}$ Glutaneurons - iCell ${ }^{\circ}$ Astrocytes co-culture (A), CNS.4U $\mathrm{U}^{\circ}$ coculture (B), SynFire iNs co-culture (C) and rat primary cortical neurons (D) before (left) and after exposure to $1 \mu \mathrm{M}$ PTX (right). Each row depicts one electrode, each tick mark represents one spike in a $200 \mathrm{~s}$ interval illustrating the pattern of activity. The cumulative trace above each plot depicts the population spike time histogram, indicating the level of synchronized activity between the different electrodes. 
A

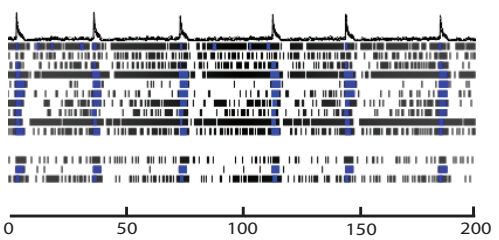

B
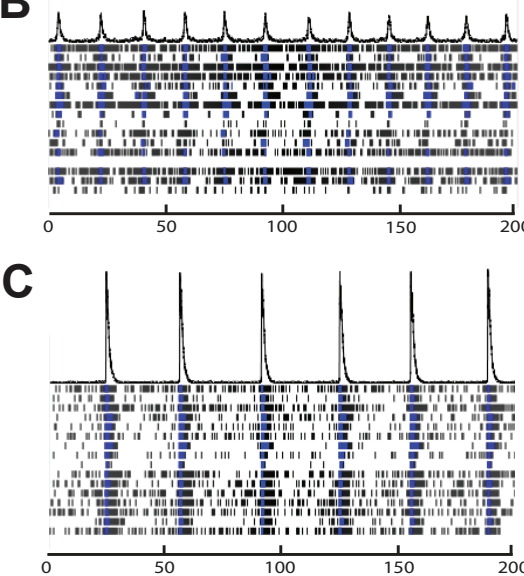

D

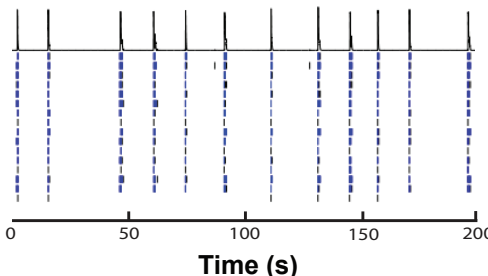

Exposure $10 \mu \mathrm{M}$

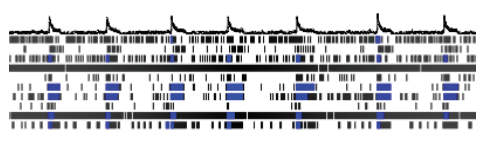

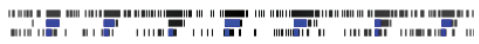
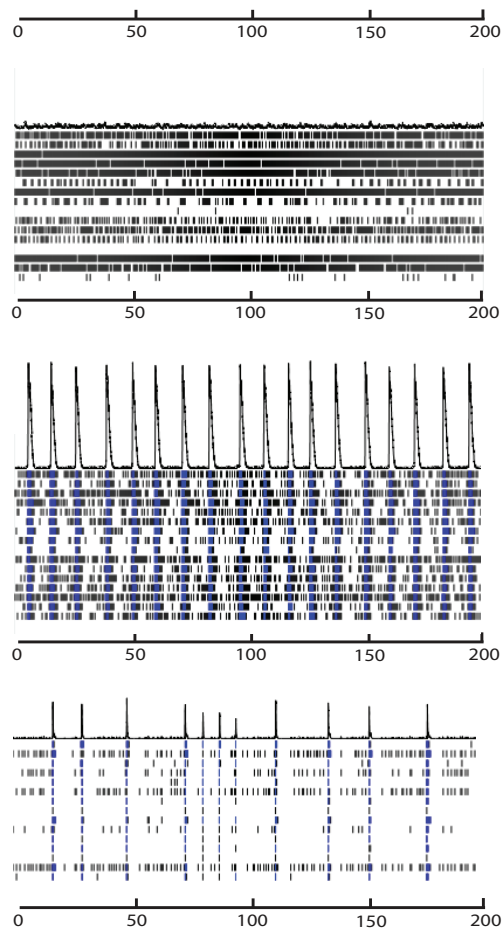

Time (s)

Figure S4. Spike raster plots of $\mathrm{Cell}^{\circ}$ Glutaneurons - iCell ${ }^{\circ}$ Astrocytes co-culture (A), CNS.4U co-culture (B), SynFire iNs co-culture (C) and rat primary cortical neurons (D) before (left) and after exposure to $10 \mu \mathrm{M}$ 4-AP (right). Each row depicts one electrode, each tick mark represents one spike in a $200 \mathrm{~s}$ interval illustrating the pattern of activity. The cumulative trace above each plot depicts the population spike time histogram, indicating the level of synchronized activity between the different electrodes. 


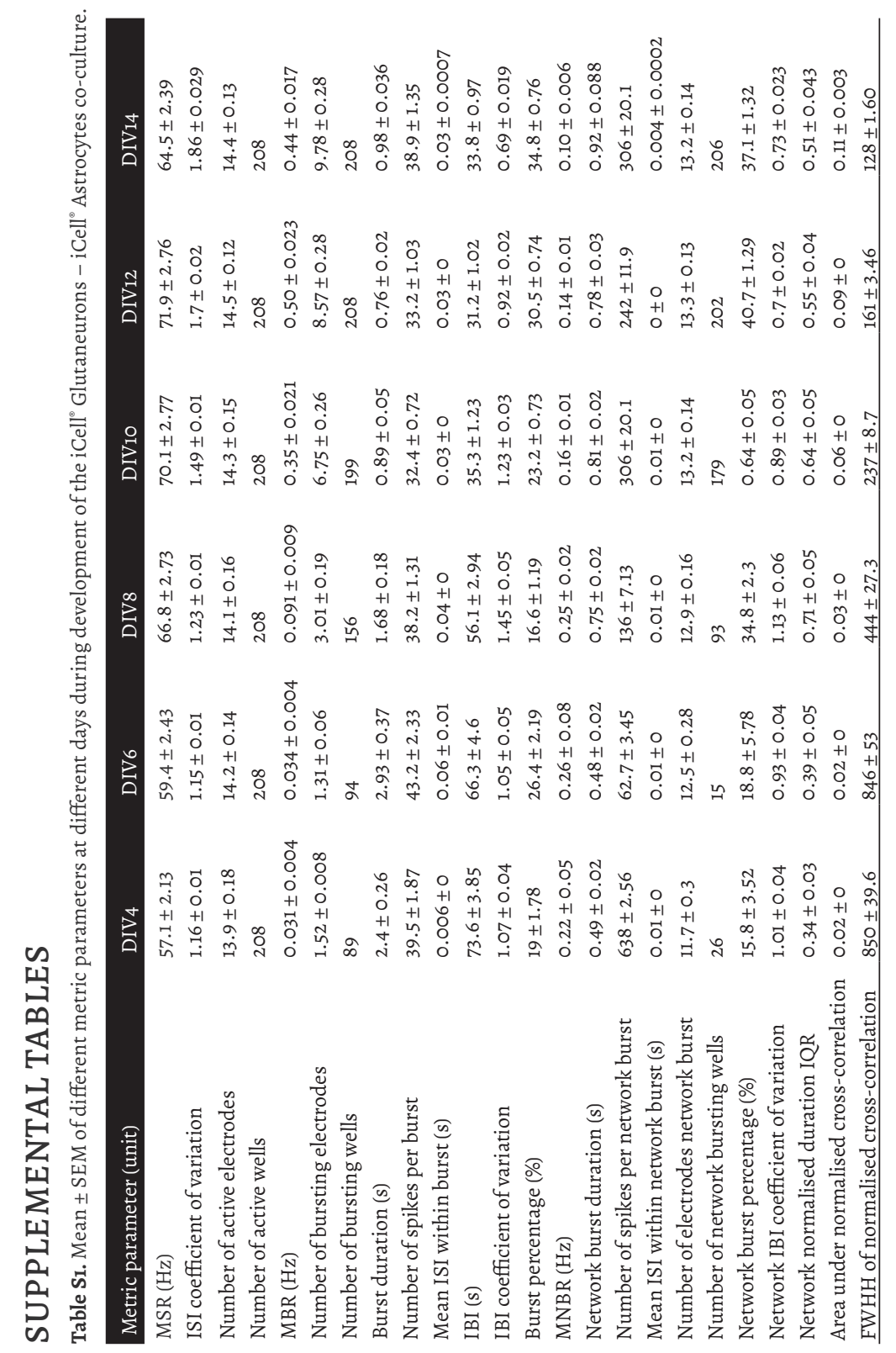




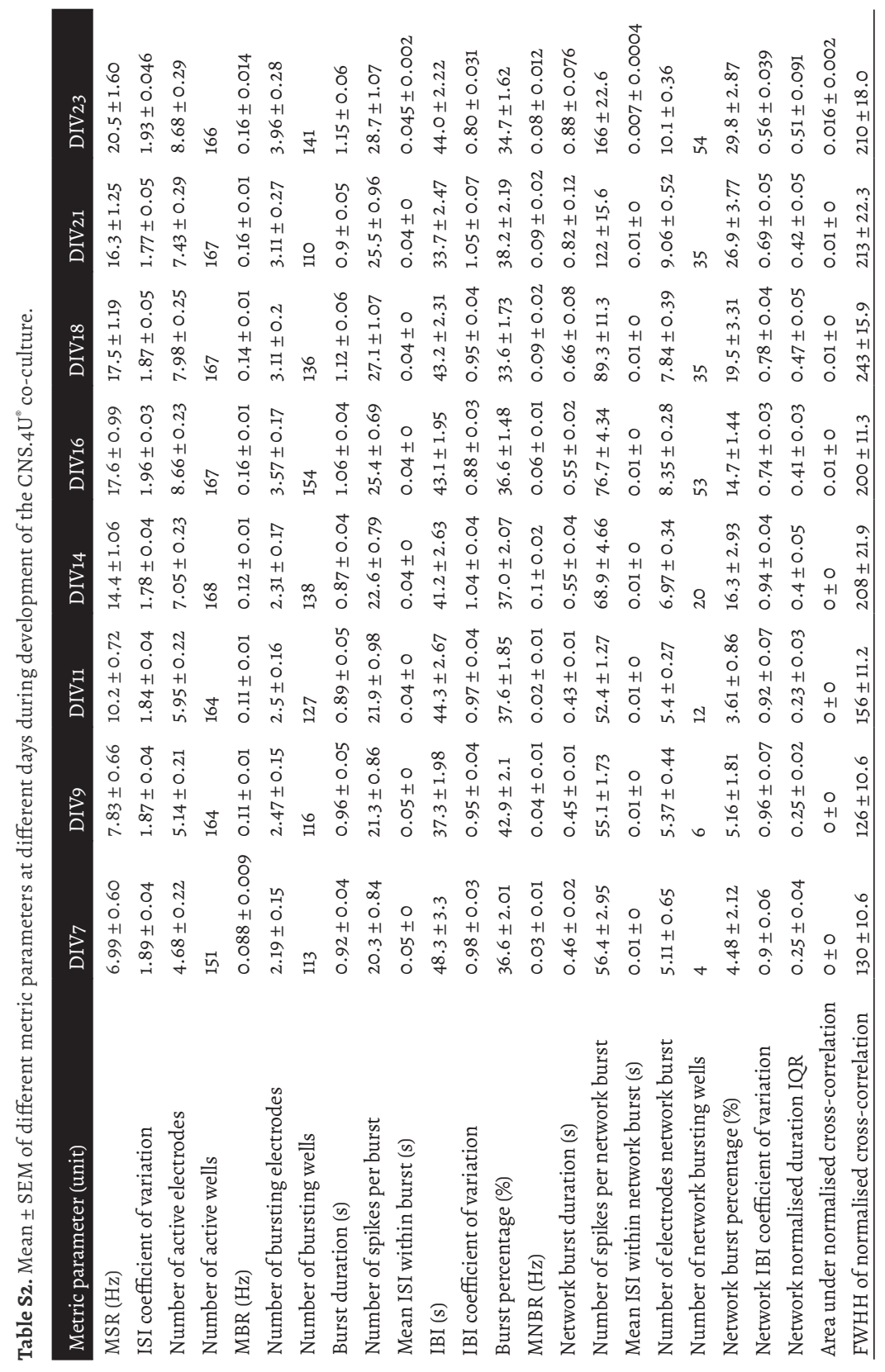




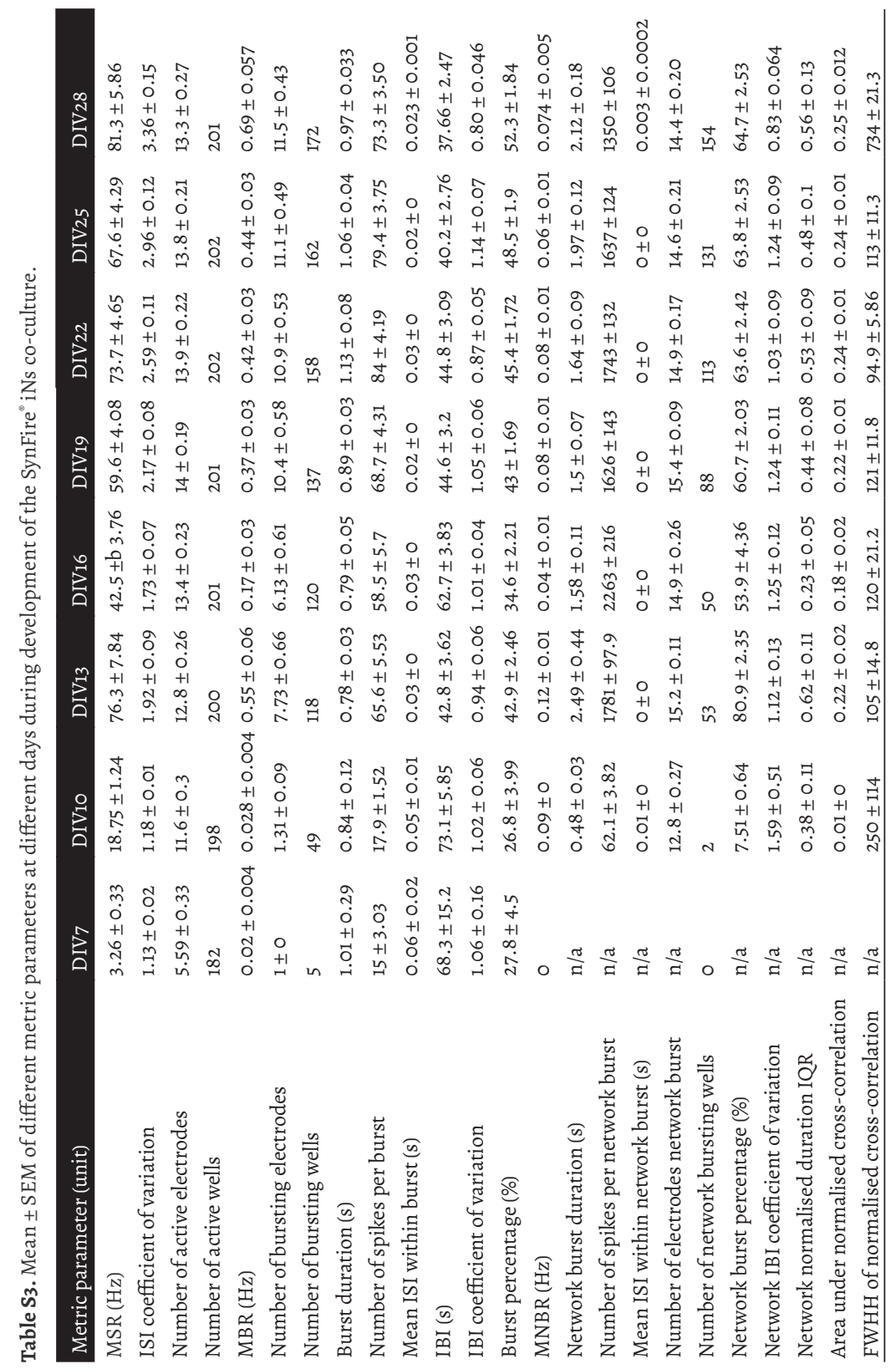


Chapter 8

\section{Applicability of hiPSC-derived neuronal co-cultures and rodent primary cortical cultures for in vitro seizure liability assessment}

Anke M. Tukker, Fiona M.J. Wijnolts, Aart de Groot and Remco H. S. Westerink

Neurotoxicology Research Group, Toxicology Division, Institute for Risk Assessment Sciences (IRAS), Faculty of Veterinary Medicine, Utrecht University, P.O. Box 80.177, NL-3508 TD Utrecht, The Netherlands.

Submitted to Toxicological Sciences, December 2019

Under revision 


\begin{abstract}
Seizures are life-threatening adverse drug reactions that are not investigated until late in drug development. Current safety assessment heavily relies on rodent models that are not always predictive for human outcome. Consequently, if seizures are detected, a lot of time, money and animals have already been used. Thus, there is a need for in vitro screening models using human cells to circumvent inter-species translation. We therefore assessed the suitability of co-cultures of human induced pluripotent stem cell (hiPSC)-derived neurons and astrocytes compared to rodent primary cortical cultures for in vitro seizure liability assessment using micro-electrode arrays (MEA).
\end{abstract}

The hiPSC-derived and rodent primary cortical neuronal co-cultures were exposed to nine known (non-)seizurogenic compounds (pentylenetetrazol (PTZ), amoxapine, enoxacin, amoxicillin, linopirdine, pilocarpine, chlorpromazine (CPZ), phenytoin and acetaminophen) to assess the effects on neuronal network activity using MEA recordings and principal component analysis (PCA). Our data indicate that all compounds, except PTZ and enoxacin, affect activity in the hiPSCderived co-culture. In rodent primary cultures all compounds, except amoxicillin and pilocarpine, changed activity. Both models had a comparable sensitivity to detect seizures following exposure to amoxapine (LOEC $1 \mu \mathrm{M}$ ). However, human iPSC-derived cultures were 10 times more sensitive for linopirdine (LOEC $3 \mu \mathrm{M}$ ) than rodent cultures (LOEC $30 \mu \mathrm{M}$ ). For (non-seizurogenic) phenytoin, the rodent cultures were more sensitive (LOEC $30 \mu \mathrm{M}$ ) than hiPSC-derived cultures (LOEC $100 \mu \mathrm{M})$. The same was true for $\mathrm{CPZ}$ where rodent cultures were 10 times more sensitive (LOEC $0.1 \mu \mathrm{M}$ ) than hiPSC-derived cultures (LOEC $1 \mu \mathrm{M}$ ).

Our data indicate that hiPSC-derived neuronal co-cultures may outperform rodent primary cortical cultures with respect to detecting seizures, thereby paving the way towards animal-free seizure assessment.

Keywords: alternatives to animal testing, human induced pluripotent stem cell (hiPSC)derived neuronal models, micro-electrode array (MEA), rodent primary cortical cultures, seizure liability assessment 


\section{INTRODUCTION}

Even though many test strategies are in place to detect adverse drug reactions, drug attrition still occurs frequently. Often this is due to central nervous system (CNS) related safety issues (Arrowsmith and Miller, 2013; Onakpoya et al., 2016). Adverse CNS effects are responsible for $\sim 10 \%$ of the drug attrition rate between 1960 and 1999 (Fung et al., 2001), emphasizing the need for continued improvement of pre-clinical test strategies. Seizures and convulsions, defined as periods of abnormal hyper-excitability of neurons that fire in a highly synchronized way (Easter et al., 2009; Jiruska et al., 2013), are a commonly encountered life threatening problem during pre-clinical drug development (Authier et al., 2016). Especially drugs that target the CNS give a higher risk on seizures due to higher brain penetration and higher affinity for CNS targets (Gao et al., 2016). However, the potential of drugs to induce seizures is not investigated until late in the drug development process during in vivo studies. If seizures are detected, the need to develop alternative compounds arises, causing delays in the drug developmental process. Also, animal experiments could have been avoided if seizure liability was detected earlier in in vitro studies. There is thus a clear need for better CNS safety testing prior to clinical studies. Hence, it would be beneficial if a reliable high throughput in vitro system becomes available for seizure liability assessment. This system would preferably use human cells to avoid inter-species translation and ethically debated animal experiments.

Currently, the rat hippocampal slice assay is one of the most used methods for in vitro seizure liability assessment (Authier et al., 2016). Brain organisation is largely maintained in this assay (Grainger et al., 2018), with active and intact neuronal networks. However, this ex vivo system is not suitable for high-throughput screens as slices have a short life-span (Buskila et al., 2015) and recordings are labour intensive requiring specific expertise and equipment (Grainger et al., 2018). Also, these brain slices are still of rodent origin. Some of these drawbacks can be resolved by multiwell micro-electrode array (mwMEA) recordings. This non-invasive tool makes it possible to record electrical activity of neuronal networks without affecting the integrity of the cells (for review see: Johnstone et al., 2010). All cell types that exhibit spontaneous activity can be cultured on mwMEAs, but rodent primary cortical cultures are the current gold standard (Authier et al., 2016). Primary cortical neurons grown on mwMEAs have many characteristics of in vivo neurons, including (spontaneous network) burst activity (Cotterill, Charlesworth, et al., 2016). The cortical cultures can be modulated with 
neurotransmitters and pharmacological and toxicological agents (Hogberg et al., 2011; McConnell et al., 2012; Nicolas et al., 2014; Hondebrink et al., 2016). It has been shown that rodent primary neuronal networks can be successfully used for seizure liability assessment (Bradley et al., 2018; Kreir et al., 2018; Fan et al., 2019; Tukker et al., 2020).

In vitro seizures can present themselves in different ways. According to Bradley et al. (2018), two patterns of in vitro seizurogenicity can be distinguished. Distinctive for pattern one is an overall increased activity, but more important is the higher synchronicity and organisation of bursts. This increased network organisation is reflected in the higher number of spikes that occur within a burst (burst percentage) and the increased inter-spike-interval coefficient of variation (ISI $\mathrm{CoV}$ ). In the case of $\mathrm{GABA}_{\mathrm{A}}$ receptor antagonists also an increase in burst duration can be observed. Picrotoxin (PTX), PTZ and 4-aminopyridine (4-AP) are examples of compounds that match this pattern (Bradley et al., 2018). Characteristics of pattern two are a decrease in activity and a deterioration of organisation and synchronicity reflected in a decreased ISI CoV, shorter bursts and longer interburst-intervals (IBIs) (Bradley et al., 2018). However, there is often only a narrow concentration window in which this pattern is visible, because of the rapid decrease in firing rate, ultimately resulting in a silent network. Examples of compounds that match this pattern are strychnine, linopirdine and amoxapine (Bradley et al., 2018).

The recent introduction of human induced pluripotent stem cell (hiPSC)-derived neurons offers an interesting opportunity to circumvent the use of animals for neurotoxicity testing. These cells lack the ethical concerns of embryonic stem cells and in vivo experiments. Human iPSC-derived neurons can be cultured on mwMEAs and develop spontaneous network activity and (network) bursting comparable to mature neurons (Odawara et al., 2016; Paavilainen et al., 2018; Sasaki et al., 2019). Also, these hiPSC-derived neuronal networks can be modulated with neurotransmitters and known neurotoxicants (Tukker et al., 2016; Odawara et al., 2018). Over the last years, an increasing number of hiPSCderived neurons became commercially available. The use of commercial iPSCs significantly reduces the time these cells need to be in culture till they develop spontaneous activity from months (Kuijlaars et al., 2016; Odawara et al., 2016) till weeks (Meneghello et al., 2015; Tukker et al., 2020). This short culture duration enhances the time- and cost-efficiency of in vitro drug screening methods. The commercial availability also reduces the potential variability between self- 
cultured batches as they can be purchased in large, quality controlled quantities (Anson et al., 2011; Little et al., 2019).

In this study, we investigated the potential of hiPSC-derived neuronal co-cultures compared to rodent primary cortical cultures for seizure liability assessment using MEA recordings.

\section{MATERIALS AND METHODS}

\subsection{Chemicals}

Neurobasal ${ }^{\circ}$-A Medium, L-glutamine, fetal bovine serum (FBS), B27 supplement (without vitamin A), N2 supplement and penicillin - streptomycin $(5000 \mathrm{U} / \mathrm{mL}$ $-5000 \mu \mathrm{g} / \mathrm{mL}$ for rat primary cortical culture media and $10.000 \mathrm{U} / \mathrm{mL}-10.000$ $\mu \mathrm{g} / \mathrm{mL}$ for hiPSC medium) were obtained from Life Technologies (Bleiswijk, The Netherlands). iCell ${ }^{\circ}$ Neural Supplement B and Nervous System Supplement were obtained from Cellular Dynamics International (Madison, WI, USA). BrainPhys ${ }^{\mathrm{TM}}$ neuronal medium was obtained from StemCell Technologies (Cologne, Germany). Ethanol (EtOH) was obtained from VWR Chemicals (Amsterdam, The Netherlands). Laminin (L2020), 50\% polyethyleneimine (PEI) solution, sodium borate, boric acid and all other chemicals (unless described otherwise) were obtained from Sigma-Aldrich (Zwijndrecht, The Netherlands).

A set of nine compounds was chosen as reference set (Table 1). An additional set of three compounds (PTX, strychnine and 4-AP; Table 1) of which data has been published previously (Tukker et al., 2020) has been added to the heatmaps for more extensive mode of action comparisons. Stock solutions of compounds dissolved in dimethyl sulfoxide (DMSO) were stored in the freezer till further use. Solutions of compounds dissolved in medium or $\mathrm{EtOH}$ were freshly prepared on the day of the experiment. 
Table 1. Compound reference set, including solvent used and reported mode(s) of action.

\begin{tabular}{|c|c|c|c|c|}
\hline & Compound & Solvent & Description & References \\
\hline \multirow{4}{*}{ 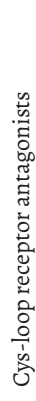 } & $\begin{array}{l}\text { Pentylenetetrazol } \\
\text { (PTZ) }\end{array}$ & Medium & $\begin{array}{l}\mathrm{GABA}_{\mathrm{A}} \text { receptor antagonist, used in } \\
\text { research as kindling model in mice and } \\
\text { rats. }\end{array}$ & $\begin{array}{l}\text { Dhir, 2012; Singh et } \\
\text { al., } 2019\end{array}$ \\
\hline & Picrotoxin $(\mathrm{PTX})^{1}$ & $\mathrm{EtOH}$ & $\begin{array}{l}\mathrm{GABA}_{\mathrm{A}} \text { receptor antagonist, used in } \\
\text { research to induce seizures }\end{array}$ & $\begin{array}{l}\text { Mackenzie et al., } \\
2002\end{array}$ \\
\hline & Amoxapine & DMSO & $\begin{array}{l}\text { Tricyclic antidepressant, blocks reuptake of } \\
\text { norepinephrine and serotonin, also blocks } \\
\text { the dopamine receptor. Reported to cause } \\
\text { seizures. }\end{array}$ & $\begin{array}{l}\text { Kapur et al., 1999; } \\
\text { Kumlien and } \\
\text { Lundberg, } 2010\end{array}$ \\
\hline & Strychnine- $\mathrm{HCl}^{1}$ & DMSO & $\begin{array}{l}\text { Glycine receptor antagonist, known to } \\
\text { induce seizures in humans and rats. }\end{array}$ & $\begin{array}{l}\text { Alachkar et al., 2018; } \\
\text { Burn et al., } 1989\end{array}$ \\
\hline \multirow{2}{*}{ 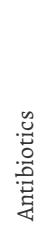 } & Enoxacin & DMSO & $\begin{array}{l}\text { Broad-spectrum antibiotic acting as } \\
\text { GABA }_{A} \text { receptor antagonist. Known to } \\
\text { trigger seizures and/or lower seizure } \\
\text { threshold. }\end{array}$ & $\begin{array}{l}\text { De Sarro et al., } \\
\text { 1993; Kawakami et } \\
\text { al., } 1997\end{array}$ \\
\hline & Amoxicillin & Medium & $\begin{array}{l}\text { Antibiotic acting as } \mathrm{GABA}_{\mathrm{A}} \text { receptor } \\
\text { antagonist. Reported to be epileptogenic. }\end{array}$ & Raposo et al., 2016 \\
\hline 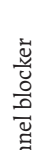 & Linopirdine & DMSO & $\begin{array}{l}\text { Kv7.x channel blocker that enhances the } \\
\text { release of neurotransmitter. Reported } \\
\text { to cause epileptic behaviour in rat } \\
\text { hippocampal slices. }\end{array}$ & $\begin{array}{l}\text { Maslarova et al., } \\
\text { 2013; Qiu et al., } 2007\end{array}$ \\
\hline 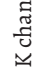 & $\begin{array}{l}\text { 4-Aminopyridine } \\
(4-\mathrm{AP})^{1}\end{array}$ & Medium & $\begin{array}{l}\text { Potassium channel blocker, used to induce } \\
\text { seizures in in vivo experiments. }\end{array}$ & $\begin{array}{l}\text { Peña and Tapia, } \\
2000\end{array}$ \\
\hline 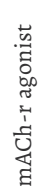 & Pilocarpine $\mathrm{HCl}$ & Medium & $\begin{array}{l}\text { Muscarinic ACh receptor agonist used as } \\
\text { eye-drop against high eye pressure. Also } \\
\text { used as in vivo model for epilepsy. }\end{array}$ & $\begin{array}{l}\text { Marchi et al., 2007; } \\
\text { Zimmerman, } 1981\end{array}$ \\
\hline \multirow{2}{*}{ 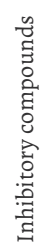 } & $\begin{array}{l}\text { Chlorpromazine } \\
\text { (CPZ) } \mathrm{HCl}\end{array}$ & Medium & $\begin{array}{l}\text { Antipsychotic drug, antagonist of } \\
\text { dopamine } \mathrm{D}_{2} \text { receptor. Reported to cause } \\
\text { seizures. }\end{array}$ & $\begin{array}{l}\text { Boyd-Kimball et al., } \\
2018 \text {; Kumlien and } \\
\text { Lundberg, } 2010\end{array}$ \\
\hline & Phenytoin & DMSO & $\begin{array}{l}\text { Anti-seizure medication by causing a block } \\
\text { of voltage gated sodium channels. }\end{array}$ & $\begin{array}{l}\text { Rogawski and } \\
\text { Löscher, } 2004\end{array}$ \\
\hline 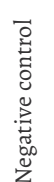 & Acetaminophen & $\mathrm{EtOH}$ & $\begin{array}{l}\text { COX- } 2 \text { inhibitor used to treat pain and } \\
\text { fever, included as negative control. }\end{array}$ & $\begin{array}{l}\text { Aminoshariae and } \\
\text { Khan, } 2015\end{array}$ \\
\hline
\end{tabular}




\subsection{Cell cultures}

Both rat primary cortical cells and hiPSC-derived neuronal co-cultures were cultured at $37^{\circ} \mathrm{C}$ in a humidified $5 \% \mathrm{CO}_{2}$ incubator. All cell culture surfaces were pre-coated with $0.1 \%$ PEI solution diluted in borate buffer $(24 \mathrm{mM}$ sodium borate/50 mM boric acid in Milli-Q adjusted to $\mathrm{pH}$ 8.4).

Primary rat cortical cells were isolated from post-natal day (PND) I Wistar rat pups (Envigo, Horst, The Netherlands) in accordance with Dutch law and approved by the Ethical Committee for Animal Experimentation of Utrecht University. Animals were treated humanely and all efforts were made to alleviate suffering. Cortical cultures were prepared as described previously (Dingemans et al., 2016; Tukker et al., 2016). Briefly, PND1 pups were decapitated and cortices were rapidly dissected on ice and kept in dissection medium (Neurobasal ${ }^{\circ}$-A supplemented with $25 \mathrm{~g} / \mathrm{L}$ sucrose, $450 \mu \mathrm{M}$ L-glutamine, $30 \mu \mathrm{M}$ glutamate, $1 \%$ penicillin/ streptomycin and 10\% FBS, pH 7.4) during the entire procedure. Cortices were dissociated to a single-cell suspension by mincing, trituration and filtering through a $100 \mu \mathrm{m}$ mesh (EASYstrainer, Greiner). The cell suspension was diluted to $2 \times 10^{6}$ cells $/ \mathrm{mL}$. Then, a $50 \mu \mathrm{L}$ droplet was placed on the electrode field of a precoated 48-well MEA plate (Axion BioSystems Inc., Atlanta, GA, USA). Cells were left for $\sim 2 \mathrm{hr}$ to adhere before adding $450 \mu \mathrm{L}$ dissection medium. To prevent glial overgrowth, $90 \%$ of the dissection medium was replaced by glutamate medium (Neurobasal ${ }^{\circ}$-A supplemented with $25 \mathrm{~g} / \mathrm{L}$ sucrose, $450 \mu \mathrm{M} \mathrm{L}$-glutamine, $30 \mu \mathrm{M}$ glutamate, $1 \%$ penicillin/streptomycin and $2 \% \mathrm{~B}-27$ supplement, $\mathrm{pH} 7.4$ ) at day in vitro (DIV) 1. At DIV4, $90 \%$ of the glutamate medium was replaced with FBS medium (Neurobasal ${ }^{\circ}$-A supplemented with $25 \mathrm{~g} / \mathrm{L}$ sucrose, $450 \mu \mathrm{M}$ L-glutamine, $1 \%$ penicillin/streptomycin and 10\% FBS, pH 7.4).

iCell $^{\oplus}$ Glutaneurons (Lot\# 103288; Cellular Dynamics International, Madison, WI, USA) and iCell $^{\circledR}$ Astrocytes (Lot\# 103956 or 11493; Cellular Dynamics International, Madison, WI, USA) were thawed and cultured according to manufacturer's protocol as described previously (Tukker et al., 2020). Briefly, cells were thawed separately in BrainPhys ${ }^{\mathrm{TM}}$ medium supplemented with $2 \% \mathrm{iCell}^{\circ}$ Neural Supplement B, 1\% Nervous System Supplement, 1\% N2, 1\% penicillin - streptomycin $(10.000 \mathrm{U} / \mathrm{mL}-10.000 \mu \mathrm{g} / \mathrm{mL})$ and $0.1 \%$ laminin. The cell pellet was dissolved in dotting medium (i.e. supplemented BrainPhys ${ }^{\mathrm{TM}}$ medium with $10 \%$ laminin). Before plating, iCell $^{\circ}$ Glutaneurons and iCell $^{\circ}$ Astrocytes were premixed into a co-culture containing $15.000 \mathrm{iCell}^{\circ}$ Glutaneurons/ $\mu \mathrm{L}$ and 6.667 $\mathrm{iCell}^{\circ}$ Astrocytes/ $\mu \mathrm{L}$ so that each well contains $120.000 \mathrm{iCell}^{\circ}$ Glutaneurons and 
$20.000 \mathrm{iCell}^{\circ}$ Astrocytes. Cells were plated in $11 \mu \mathrm{L}$ droplets (140.000 cells/droplet with $85 \%$ iCell $^{\circ}$ Glutaneurons and $15 \%$ iCell $^{\circ}$ Astrocytes) over the electrode field of 48-well MEA plates. Following plating, cells were allowed to adhere for $\sim \mathrm{hhr}$ after which $300 \mu \mathrm{L}$ of room temperature (RT) supplemented BrainPhys ${ }^{\mathrm{TM}}$ medium was added. Hereafter, $50 \%$ medium changes with RT supplemented BrainPhys ${ }^{\mathrm{TM}}$ medium took place at DIV1, 2, 4, 6, 8, 10, 12 and 14 .

\subsection{MEA recordings}

Each well of a 48-well MEA plate contains 16 nanotextured gold micro-electrodes ( $\sim 40-50 \mu \mathrm{m}$ diameter; $350 \mu \mathrm{m}$ spacing) with 4 integrated ground electrodes. This yields a total of 768 channels that can be recorded simultaneously (for review see: Johnstone et al., 2010). Spontaneous electrical activity was recorded as described previously (Nicolas et al., 2014; Tukker et al., 2019). Briefly, signals were recorded at the day of experiments (DIV9-11 for rat primary cortical cultures or DIV14 for hiPSC-derived co-cultures) using a Maestro 768-channel amplifier with integrated heating system and temperature controller and a data acquisition interface (Axion BioSystems Inc., Atlanta, GA, USA). Data acquisition was managed with Axion's Integrated Studio (AxIS 2.4.2.13) and recorded as .RAW files. All channels were sampled simultaneously with a gain of $1200 \mathrm{x}$ and a sampling frequency of $12.5 \mathrm{kHz} /$ channel, using a 200-5000 Hz band-pass filter. Prior to the recording, MEA plates were allowed to equilibrate for 5-10 min in the Maestro.

In order to determine the effects of reference compounds on spontaneous neuronal activity (spiking and (network) bursting behaviour) on the two types of cell cultures, a $30 \mathrm{~min}$ baseline recording was made. Following this recording, wells were exposed (10 x dilution for primary rat cortical cultures, $30 \mathrm{x}$ dilution for hiPSC-derived co-cultures) to the reference compounds or the appropriate solvent control. Then activity was recorded for another $30 \mathrm{~min}$. Stock solutions of compounds dissolved in DMSO or EtOH were diluted in culture medium to obtain desired concentrations. In all experiments, the solvent concentration never exceeded $0.1 \% \mathrm{v} / \mathrm{v}$. In order to prevent receptor (de)sensitization, each well was exposed to a single concentration. For each experimental condition, MEA plates from at least two different plating rounds were used.

\subsection{Data analysis and statistics}

To determine (modulation of) spontaneous activity, .RAW data files were rerecorded to obtain Alpha Map files for further data analysis. In this re-recording, spikes were detected using the AxIS spike detector (Adaptive threshold crossing, 
Ada BandFIt v2) with a variable threshold spike detector set at $7 \mathrm{x}$ (primary cortical culture) or 5.5x (hiPSC culture) standard deviation (SD) of internal noise level (rms) on each electrode. Post/pre-spike duration was set to 3.6/2.4 ms. For further analysis, spike files were loaded in NeuralMetric Tool (version 2.2.4, Axion BioSystems). Only active electrodes (MSR $\geq 6$ spikes/min) in active wells ( $\geq 1$ active electrode) were included in the data analysis. The (network) bursting behaviour was analyzed as it is crucial for (in vivo) transmission of information (Izhikevich et al., 2003) using the Poisson Surprise method (Legéndy and Salcman, 1985) with a minimal surprise of 10 and a minimum bursting frequency of 0.3 bursts $/ \mathrm{min}$. Network bursts were extracted with the adaptive threshold algorithm.

Effects of reference compounds on the spontaneous activity pattern were determined in exposure experiments where baseline activity prior to exposure was compared with activity following exposure. To prevent inclusion of exposure artefacts, windows for data analysis were corrected for the time it took to expose the whole plate. In other words, if exposure took $2 \mathrm{~min}$, the first 2 min were not included in data analysis or when a window of 20-30 min post exposure was analyzed, analysis started at $20+2 \mathrm{~min}$. Optimum effect windows were determined separately for all reference compounds. For all reference compounds the window of 20-30 min post exposure was used for analysis of effects.

A custom-made MS Excel macro was used to calculate treatment ratios (TR) per well for the different metric parameters (Table 2) by: (parameter ${ }_{\text {exposure }}$ l parameter $_{\text {baseline }}$ ) $100 \%$. Hereafter, TRs were normalized to appropriate vehicle control. Outliers defined as not within average $\pm 2 \times$ SD were removed $(3.4 \%$ for hiPSC-derived neuronal data and $4.8 \%$ for rat primary cortical data). To test for concentration-dependent effects a one-way Welch's ANOVA was performed. If the outcome of this test was significant, a post-hoc Dunnett's test was performed. For both tests, $p$-values $<0.05$ were considered significant. All statistical analyses were performed in $\mathrm{R}$ version 3.6.0 ( $\mathrm{R}$ core team 2019) using the DescTools package (by Signorell et al., 2019, version 0.99.28). Heatmaps were also created in R using the pheatmap package (by Kolde, 2019, version 1.0.12). Data are presented as mean \pm SEM from the number of wells ( $\mathrm{n}$ ) indicated, derived from at least 2 independent plating rounds. Lowest observed effect concentrations (LOECs) are defined as lowest statistically significant concentrations at one of the activity parameters. 
Table 2. Description of different metric parameters. Adapted from Tukker et al., 2020. Parameters in italic are included in the principal component analysis.

\begin{tabular}{|c|c|c|}
\hline \multirow{3}{*}{ 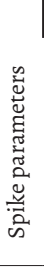 } & Metric parameter & Description \\
\hline & Mean spike rate (MSR) & $\begin{array}{l}\text { Total number of spikes divided by recording time } \\
(\mathrm{Hz}) \text {. }\end{array}$ \\
\hline & $\begin{array}{l}\text { Inter-spike interval (ISI) coefficient of variation } \\
(\mathrm{CoV})\end{array}$ & $\begin{array}{l}\text { Standard deviation ISI (time between spikes) } \\
\text { divided by the mean ISI. Measure for spike } \\
\text { regularity: } 0 \text { indicates perfect spike distribution, } \\
>1 \text { signals bursting. }\end{array}$ \\
\hline \multirow{9}{*}{ 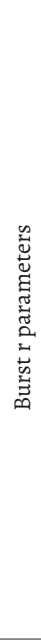 } & Mean burst rate (MBR) & $\begin{array}{l}\text { Total number of bursts divided by recording time } \\
(\mathrm{Hz}) \text {. }\end{array}$ \\
\hline & Burst duration & $\begin{array}{l}\text { Average time from the first spike in a burst till the } \\
\text { last spike (s). }\end{array}$ \\
\hline & Number of spikes per burst & Average number of spikes occurring in a burst. \\
\hline & Mean ISI within burst & Mean ISI within a burst (s). \\
\hline & Median ISI within burst & Median ISI within a burst (s). \\
\hline & Median / mean ISI within burst & $\begin{array}{l}\text { Median / mean ISI within a burst. Values close to I } \\
\text { indicate a symmetric distribution of spikes within } \\
\text { a burst. }\end{array}$ \\
\hline & Inter-burst interval (IBI) & $\begin{array}{l}\text { Time between the last spike of a burst and the first } \\
\text { spike of a subsequent burst (s). }\end{array}$ \\
\hline & IBI CoV & $\begin{array}{l}\text { Standard deviation of IBI divided by the mean IBI. } \\
\text { Measure for burst regularity. }\end{array}$ \\
\hline & Burst percentage & $\begin{array}{l}\text { Percentage of total number of spikes occurring } \\
\text { in a burst. }\end{array}$ \\
\hline \multirow{4}{*}{ 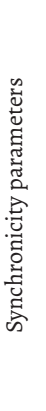 } & Area under normalised cross-correlation & $\begin{array}{l}\text { Area under inter-electrode cross-correlation } \\
\text { normalized to the auto-correlations. The higher } \\
\text { the value, the greater the synchronicity of the } \\
\text { network. }\end{array}$ \\
\hline & Area under cross-correlation (AUC) & Area under inter-electrode cross-correlation. \\
\hline & $\begin{array}{l}\text { Full width at half height (FWHH) of } \\
\text { normalized cross-correlation }\end{array}$ & $\begin{array}{l}\text { Width at half left height of the normalized cross- } \\
\text { correlogram to half right height. Measure for } \\
\text { network synchrony: the higher the value, the less } \\
\text { synchronized the network is. }\end{array}$ \\
\hline & FWHH of cross-correlation & $\begin{array}{l}\text { Width at half left height of the cross-correlogram } \\
\text { to the half right height. }\end{array}$ \\
\hline
\end{tabular}


Mean network burst rate (MNBR)

Network burst duration

Number of spikes per network burst

Mean ISI within network burst

Median ISI within network burst

Number of electrodes participating in network burst

Number of spikes per network burst per channel

Network burst percentage

Network IBI CoV

Network normalised duration IQR
Total number of network bursts divided by recording time $(\mathrm{Hz})$.

Average time from the first spike till the last spike in a network burst (s).

Average number of spikes occurring in a network burst.

Average of the mean ISIs within a network burst (s).

Median ISI within a network burst (s).

Median / mean ISI within a network burst. Values close to 1 indicate a symmetric distribution of spikes within a burst.

Average number of electrodes with spikes that participate in the network burst.

Average number of spikes in a network burst, divided by the number of electrodes that participate in the network burst.

Percentage of total spikes occurring in a network burst.

Standard deviation of network IBI divided by the mean network IBI. Measure of network burst rhythmicity: value is small when bursts occur at regular interval and increases when bursts occur more sporadic.

Interquartile range of network bursts durations. Measure for network burst duration regularity: larger values indicate wide variation in duration.

\subsection{Principal component analysis}

Principal component analysis (PCA) can be used to visualise the level of variation between different culture models following exposure to compounds with the same mode of action. Therefore, a PCA was performed to segregate MEA results of hiPSC-derived co-cultures and rat primary cortical cultures following exposure to $\mathrm{GABA}_{\mathrm{A}}$ antagonists (PTZ, PTX, amoxapine, enoxacin and amoxicillin) and visualise a (potential) inter-species difference. The class of $\mathrm{GABA}_{\mathrm{A}}$ antagonists was chosen, because they form the majority of compounds in this study. The PCA was conducted in $\mathrm{R}$ using the packages FactoMineR (Lê et al., 2008) and FactoExtra (by Mundt, 2017; version 1.0.5). To prevent empty cells in the matrix, average 
values per parameter of the middle test concentration were calculated. Next, highly correlated parameters were removed from the data set. Correlation was determined based on visual inspection of the correlation matrix and the heatmap, resulting in removal of 10 parameters. The resulting 14 parameters included in the analysis are depicted in italic in Table 1, including MSR, MBR, MNBR, parameters related to (network) burst duration and percentage and synchronicity parameters. A scree plot was created to visualise the percentage of variation explained by each component and eigen-values were calculated. The loading of each parameter to a component was calculated. Parameters contributing more than the expected contribution of $7 \%$ (expected value $=(1 /$ number of variables $\mathrm{x}$ $100 \%)$ ) were considered important for a component. The 14-dimensional vector was projected on the plane created by the first 2 principal components that explained most of the variation to visualise the level of (potential) inter-species variation between the two models.

\section{RESULTS}

\subsection{Effects on spontaneous network activity}

In a previous study we have showed that hiPSC-derived neuronal co-cultures and the rat primary cortical cultures develop spontaneous activity with (network) bursting behaviour (Tukker et al., 2020). The average mean spike rate is $64.5 \pm$ $2.39 \mathrm{~Hz}$ for hiPSC-derived neuronal co-cultures and $21.4 \pm 0.82 \mathrm{~Hz}$ for rat primary cortical cultures. The mean burst rate is on average $0.44 \pm 0.017 \mathrm{~Hz}$ and $0.77 \pm$ 0.028 , whereas the mean network burst rate is $0.1 \pm 0.006 \mathrm{~Hz}$ and $0.073 \pm 0.002$ $\mathrm{Hz}$ for hiPSC-derived neuronal co-cultures and rat primary cortical cultures, respectively (Tukker et al., 2020). To assess and compare the suitability of hiPSCderived neuronal co-cultures and rat primary cortical cultures for seizure liability assessment, both models were exposed to compounds with different modes of action (Table 1). Observed neurotoxic effects were not caused by cytotoxicity (see supplemental data Fig. S1).

As a negative control, cells were exposed to acetaminophen. Exposure to this compound did not result in changes in neuronal activity in rat primary cortical cultures (Zwartsen et al., 2019) or hiPSC-derived neuronal co-cultures (supplemental data Fig. S2). 


\subsubsection{Cys-loop receptor antagonists}

PTZ increases the MSR in rat primary cortical cultures, but has little effect on the hiPSC-derived neuronal co-culture (Fig. IA; left). The MBR increases in both cultures following exposure, but only significantly in the rat primary cortical culture (Fig. 1A; middle). Both models show an increased MNBR, but this increase is not significant (Fig. 1A; right).

Amoxapine increases the MSR significantly in both cultures, but at the highest concentration tested, a significant decrease in activity occurs (Fig. 1B; left). The same trend occurs in the MBR (Fig. 1B; middle) and MNBR (Fig. 1C; right). Although for the latter, the change is not significant. Overall, exposure to amoxapine increases activity in both models, but activity decreases following exposure to $10 \mu \mathrm{M}$.
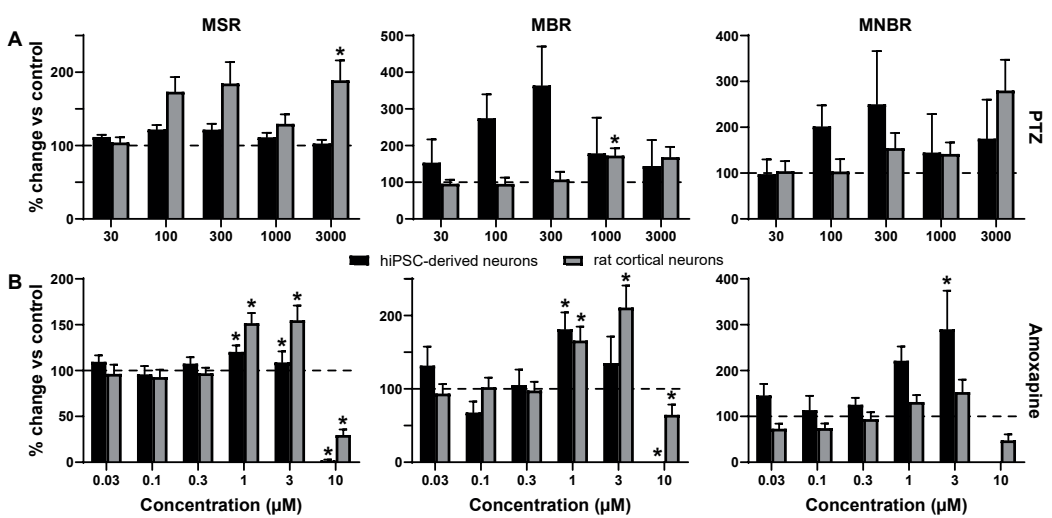

Figure 1. Effects of modulation of hiPSC-derived co-cultures (black) and rat primary cortical cultures (grey) with PTZ (A) and amoxapine (B) on mean spike rate (MSR; left), mean burst rate (MBR; middle) and mean network burst rate (MNBR; right). Effects are depicted as average \% change of control (solvent control set to $100 \%$; dashed line) \pm SEM from $n=4$ - 13 wells for hiPSC-derived neuronal cocultures and $\mathrm{n}=13-33$ wells for rat primary cortical cultures. ${ }^{*} p<0.05$.

\subsubsection{Antibiotics}

MSR of hiPSC-derived neuronal co-cultures is not affected following exposure to enoxacin (Fig. 2A; left). However, a statistically significant increase can be observed when rat primary cortical cultures are exposed. Enoxacin has little effect on MBR of hiPSC-derived co-cultures, but increases MBR significantly at the highest test concentration $(10 \mu \mathrm{M})$ in rat primary cortical cultures (Fig. 2A; middle). At low test concentrations, enoxacin decreases MNBR in hiPSC-derived 
co-cultures, but not significantly (Fig. 2A; right). In rat primary cortical cultures MNBR increases, however not significantly.

Amoxicillin has no effect on MSR or MNBR in either of the models (Fig. 2B; left/ right respectively), but increases MBR significantly in hiPSC-derived co-cultures (Fig. 2B; middle).
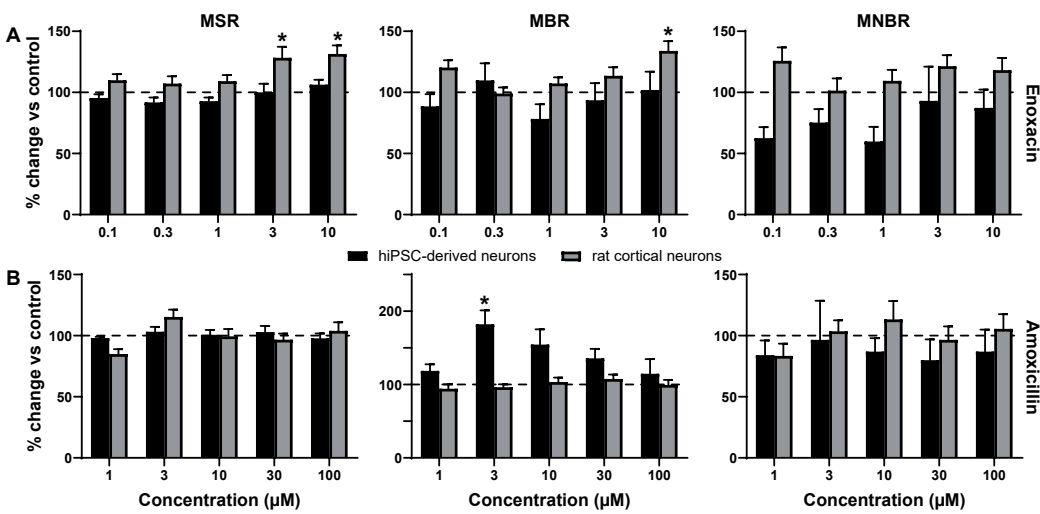

Figure 2. Effects of modulation of hiPSC-derived co-cultures (black) and rat primary cortical cultures (grey) with enoxacin (A) and amoxicillin (B) on mean spike rate (MSR; left), mean burst rate (MBR; middle) and mean network burst rate (MNBR; right). Effects are depicted as average \% change of control (solvent control set to $100 \%$; dashed line) \pm SEM from $\mathrm{n}=10-12$ wells for hiPSCderived neuronal co-cultures and $n=26-35$ wells for rat primary cortical cultures. ${ }^{*} p<0.05$.

\subsubsection{Muscarinic acetylcholine receptor agonist}

Pilocarpine significantly increases MSR in hiPSC-derived neuronal co-cultures following exposure to $1 \mu \mathrm{M}$ and significantly decreases spike activity following exposure to $30 \mu \mathrm{M}$ (Fig. 3A). In rat primary cortical cultures MSR decreases, but this change is not statistically significant. An increase followed by a significant decrease at $30 \mu \mathrm{M}$ in $\mathrm{MBR}$ can be observed following pilocarpine exposure on hiPSC-derived co-cultures (Fig. 3B). In rat primary cortical cultures exposure with low concentration results in a decrease of burst activity, whereas at higher concentrations there is little effect. Pilocarpine has little effect on MNBR of hiPSC-derived co-cultures, but increases network bursting in rat primary cortical cultures following exposure to 3 and $10 \mu \mathrm{M}$ (Fig. 3C). However, this change is not significant. 


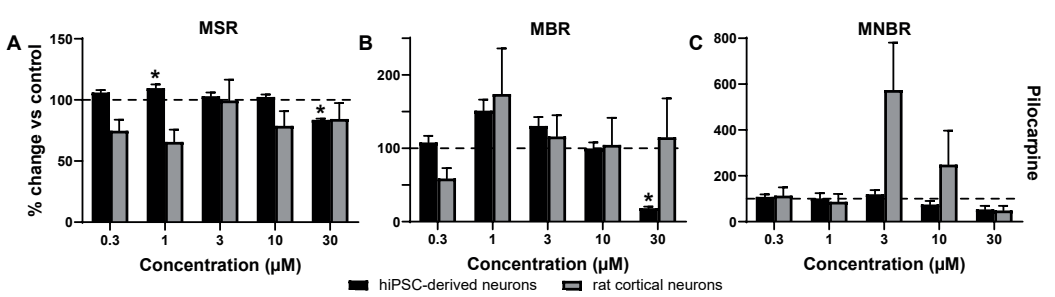

Figure. 3. Effects of modulation of hiPSC-derived co-cultures (black) and rat primary cortical cultures (grey) with pilocarpine on mean spike rate (MSR;A), mean burst rate (MBR; B) and mean network burst rate (MNBR;C). Effects are depicted as average \% change of control (solvent control set to $100 \%$; dashed line) \pm SEM from $n=9-12$ wells plates for hiPSC-derived neuronal co-cultures and $\mathrm{n}=15-24$ wells for rat primary cortical cultures. ${ }^{*} p<0.05$.

\subsubsection{Potassium channel blocker}

Linopirdine significantly decreases MSR in hiPSC-derived co-cultures, but not in rat primary cortical cultures (Fig. 3A). MBR increases significantly following exposure to $3 \mu \mathrm{M}$ linopirdine in hiPSC-derived co-cultures, followed by a decrease at higher test concentrations (Fig. 3B). This trend is not observed in rat primary cortical cultures where MBR is not affected by linopirdine. At low concentrations linopirdine increases MNBR of hiPSC-derived co-cultures, but this is followed by a decrease at higher concentrations (Fig. $3 \mathrm{C}$ ). None of these changes are statistically significant. In rat primary cortical cultures MNBR increases and the increase is significant at $30 \mu \mathrm{M}$.
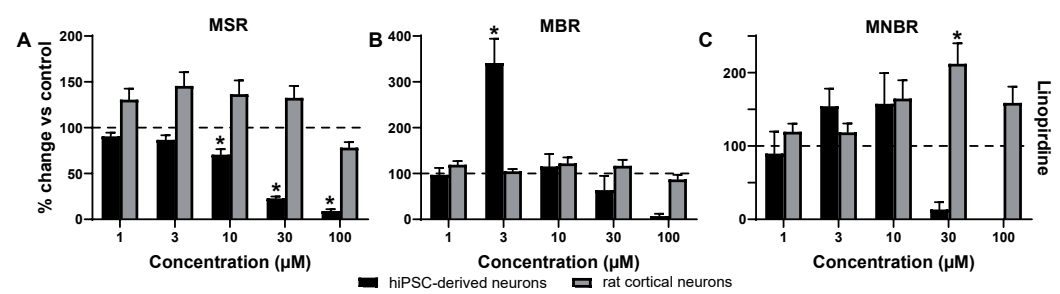

Figure 4. Effects of modulation of hiPSC-derived co-cultures (black) and rat primary cortical cultures (grey) with linopirdine on mean spike rate (MSR;A), mean burst rate (MBR; B) and mean network burst rate (MNBR;C). Effects are depicted as average \% change of control (solvent control set to $100 \%$; dashed line) \pm SEM from $n=7$ - 12 wells plates for hiPSC-derived neuronal co-cultures and $\mathrm{n}=26-29$ wells for rat primary cortical cultures. ${ }^{*} p<0.05$. 


\subsubsection{Inhibitory compounds}

CPZ increases MSR significantly in both models followed by a decrease at higher test concentrations (Fig. 5 A; left). Exposure of both models to low concentrations of $\mathrm{CPZ}$ does not affect bursting, however at a high concentration there is a significant decrease of MBR (Fig. 5A; middle). MNBR decreases following exposure in hiPSC-derived co-cultures, but significantly decreases in rat primary cortical cultures (Fig. 5A; right).

Phenytoin significantly decreases MSR in both models at high test concentration (Fig. 5B; left). At low test concentrations burst activity increases in both models. However, this increase is not significant (Fig. 5B; middle). A significant decrease in MBR can be observed at the highest test concentration $(100 \mu \mathrm{M})$ in the rat primary cortical culture. MNBR increases following phenytoin exposure in hiPSC-derived neuronal co-culture (Fig. 5B; right). In rat primary cortical cultures, MNBR decreases.
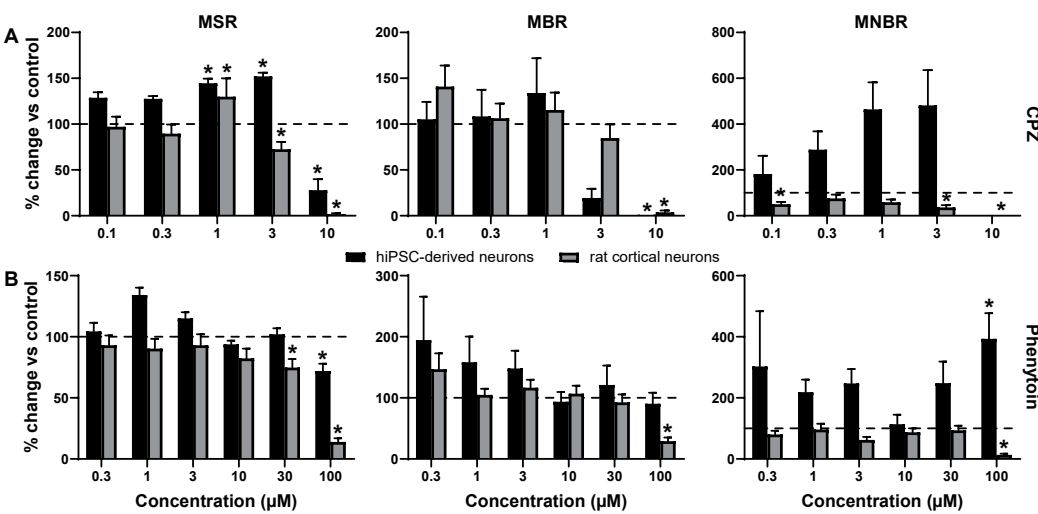

Figure 5. Effects of modulation of hiPSC-derived co-cultures (black) and rat primary cortical cultures (grey) with CPZ (A) and phenytoin (B) on mean spike rate (MSR; left), mean burst rate (MBR; middle) and mean network burst rate (MNBR; right). Effects are depicted as average \% change of control (solvent control set to $100 \%$; dashed line) \pm SEM from $n=4-16$ wells plates for hiPSC-derived neuronal co-cultures and $\mathrm{n}=21-32$ wells for rat primary cortical cultures. ${ }^{*} p<0.05$. 


\subsection{Finger prints of the different compound classes}

Heatmaps of the concentration-response curve of all tested compounds on hiPSC-derived neuronal co-cultures and rodent primary cortical cultures were created to further illustrate the effect on different parameters. These heatmaps also visualise that compounds with the same mode of action have a comparable chemical fingerprint (Fig 6-7). Because these heatmaps show more parameters than just the classical activity parameters as depicted in Fig. 1-5, they create a completer overview of how a network is affected by exposure. For example, when looking at the heatmaps, it is immediately clear that parameters such as (network) burst percentage, mean and median ISI within (network) burst and area under cross correlation are strongly affected by exposure to these (non-) seizurogenic compounds. These heatmaps thus provide a valuable tool for visualising changes in neuronal networks following modulation, including potential seizurogenicity patterns as described by Bradley et al., 2018.

When the hiPSC-derived neuronal co-cultures are exposed to PTZ or PTX, both $\mathrm{GABA}_{\mathrm{A}}-\mathrm{R}$ antagonists, a comparable pattern occurs and strongest effects can be seen on burst related parameters (Fig 6A top). However, effects are more profound following exposure to PTZ. Exposure of hiPSC-derived co-cultures to amoxapine, also a $\mathrm{GABA}_{\mathrm{A}}-\mathrm{R}$ antagonist, increases MSR, MBR, MNBR, network burst duration and the area under cross correlation, just like PTZ and PTX. However, the strong increasing effect on burst related parameters is not so clear following exposure to amoxapine. The fourth compound in the cys-loop receptor antagonist category, strychnine, shows a different pattern with a clear decrease in activity when exposed to hiPSC-derived co-cultures. This is also the only compound with a different target, the glycine receptor. Exposure of rat primary cortical cultures to the same set of cys-loop receptor antagonists yields a pattern different from the pattern of hiPSC-derived neuronal co-cultures (Fig. 6A bottom). Overall, activity decreases following exposure of rat primary cortical cultures. The pattern for all four compounds is comparable, with increases in MSR, MBR, MNBR and the area under cross correlation.

The next group consists of two antibiotics, both $\mathrm{GABA}_{\mathrm{A}}$-receptor antagonists. When looking at the pattern resulting from exposure of hiPSC-derived cocultures it can be seen that it is comparable with the pattern following exposure to the GABA-receptor antagonists PTZ and PTX, but effects are milder (Fig 6B top). Especially exposure to amoxicillin results in a very comparable pattern. The same holds true for rat primary cortical cultures. When these cells are exposed 
to enoxacin or amoxicillin, a comparable, but milder, pattern as in the cys-loop receptor antagonists occurs (Fig 6B bottom).

Exposure of both models to the mACh-R agonist pilocarpine results in a pattern that indicates this different mode of action as it has little similarities with other compounds (Fig. 7A). There are also little similarities between the pattern in hiPSC-derived neuronal co-cultures and rat primary cortical co-cultures.

Two potassium channel blockers were tested (Fig. 7B). For neither cell model it is clear from the heatmap that the compounds have the same mode of action. In both cell models, linopirdine affects network burst parameters strongest, whereas 4-AP has more effect on burst related parameters.

The last group consists of two inhibitory compounds. CPZ has the D2-receptor as target and strongly increases $M B R, M N B R$ and network burst percentage and decreases the IBI in hiPSC-derived neuronal networks (Fig. 7C). This same increase can be observed when hiPSC-cells are exposed to phenytoin, a sodium channel blocker. Both compounds have relatively mild effects on spike parameters. When rat primary cortical cultures are exposed to $\mathrm{CPZ}$ or phenytoin, spike parameters decrease. At the lowest test concentrations there is an increase of MBR, but bursting decreases with increasing concentration. The MNBR decreases. Overall, effects on rat primary cortical cultures are milder than on hiPSC-derived co-cultures.

Figure 6. Heatmap of the effects of A) cys-loop antagonists (green; PTZ, PTX, amoxapine and strychnine) and B) antibiotics (orange; enoxacin and amoxicillin) on hiPSC-derived neuronal cocultures (black) and rat primary cortical cultures (grey). Color scaling is based on the percentage of change relative to the control with red indicating an increase and blue a decrease. White areas indicate that no average could be calculated. Data is based on $n=1-18$ wells for hiPSC-derived neuronal co-cultures and $\mathrm{n}=9-35$ wells for rat primary cortical cultures. PTX and strychnine data have been published previously in Tukker et al., 2020. 


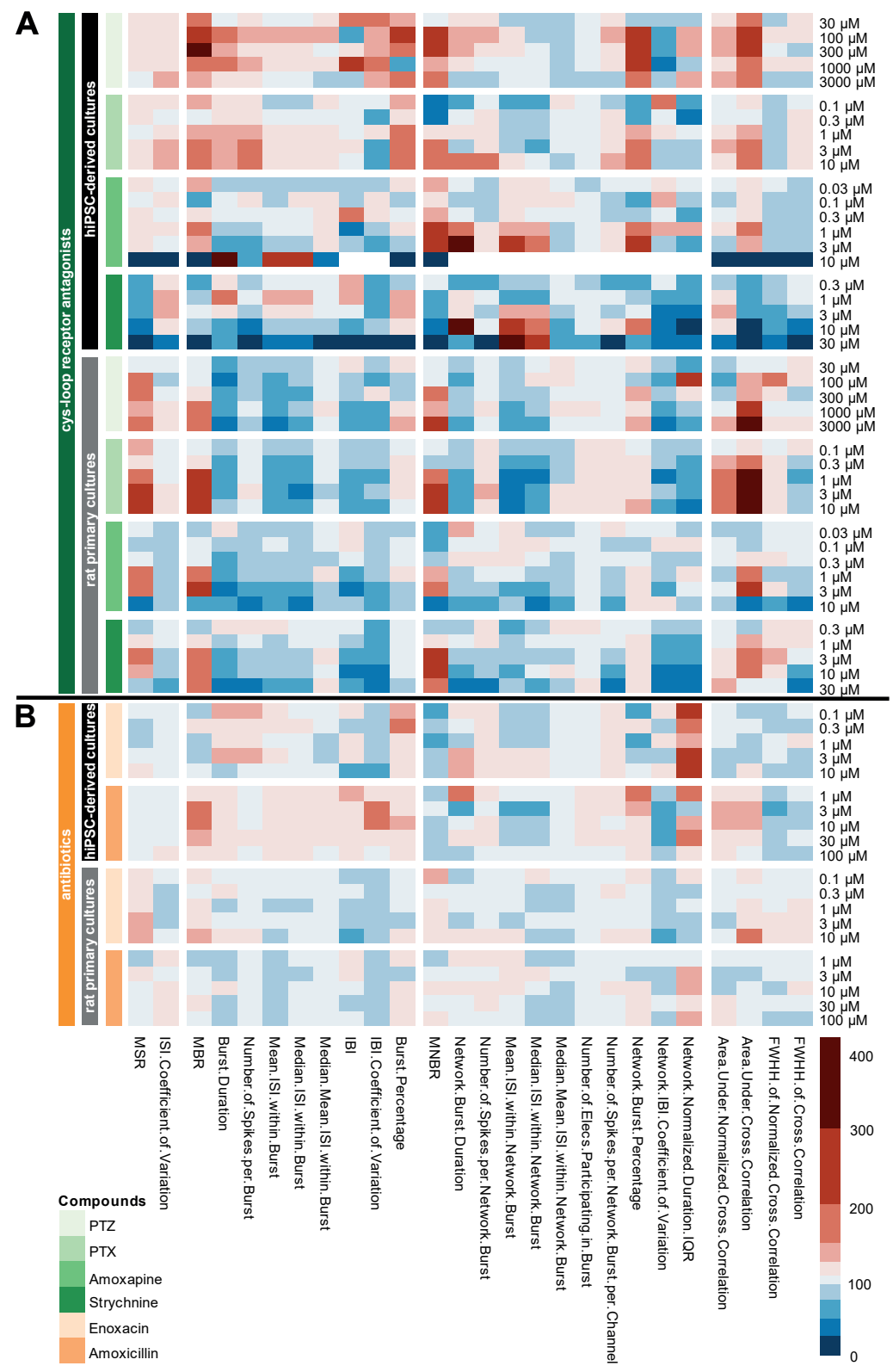




\subsection{Principal component analysis to distinguish between human and rodent model}

In order to visualise the potential inter-species differences following exposure to $\mathrm{GABA}_{\mathrm{A}}-\mathrm{R}$ antagonists between the hiPSC-derived neuronal co-culture and rat primary cortical culture, a PCA was performed. PCA efficiently clusters MEA data based on the activity profile of the networks following exposure.

A PCA was performed on 10 items (5 compounds per model) and 14 parameters. Four principal components have an eigenvalue greater than 1. Together, these components explain $92.2 \%$ of the variability as becomes clear from the scree plot (Fig. 8A). In total, 8 parameters score higher than the expected $7 \%$ contribution to component 1 (Fig. 8B) and 5 parameters for component 2 (Fig. 8C). What is interesting to note is that the most important parameters contributing to PCA1 relate to duration of (network) bursts and the number of spikes in bursts. This is in contrast to PCA2 where classical activity parameters (mean burst rate and mean network burst rate) as well as parameters related to synchronicity are more important. Interestingly, the mean spike rate has a negative contribution to PCA1 (Fig. 8D). What becomes clear from the biplot (Fig. 8D) is that there is a clear segregation between data points from hiPSC-derived neuronal co-cultures and rat primary cortical neurons (Fig. 8D). With the exception of amoxicillin on rat primary cortical cultures, all rat primary cortical culture data is presented in left quadrants of the graph, whereas the hiPSC-derived neuronal co-culture data is presented in the right quadrants.

Figure 7. Heatmap of the effects of A) a muscarinic acethylcholine receptor agonist (blue; pilocarpine), B) potassium channel blockers (purple; linopirdine and 4-AP) and C) inhibitory compounds (red; CPZ and phenytoin) on hiPSC-derived neuronal co-cultures (black) and rat primary cortical cultures (grey). Rpc means rat primary cultures. Color scaling is based on the percentage of change relative to the control with red indicating an increase and blue a decrease. White areas indicate that no average could be calculated. Data is based on $n=2-16$ wells for hiPSC-derived neuronal co-cultures and $\mathrm{n}=95-31$ wells for rat primary cortical cultures. 4-AP data have been published previously in Tukker et al., 2020. 


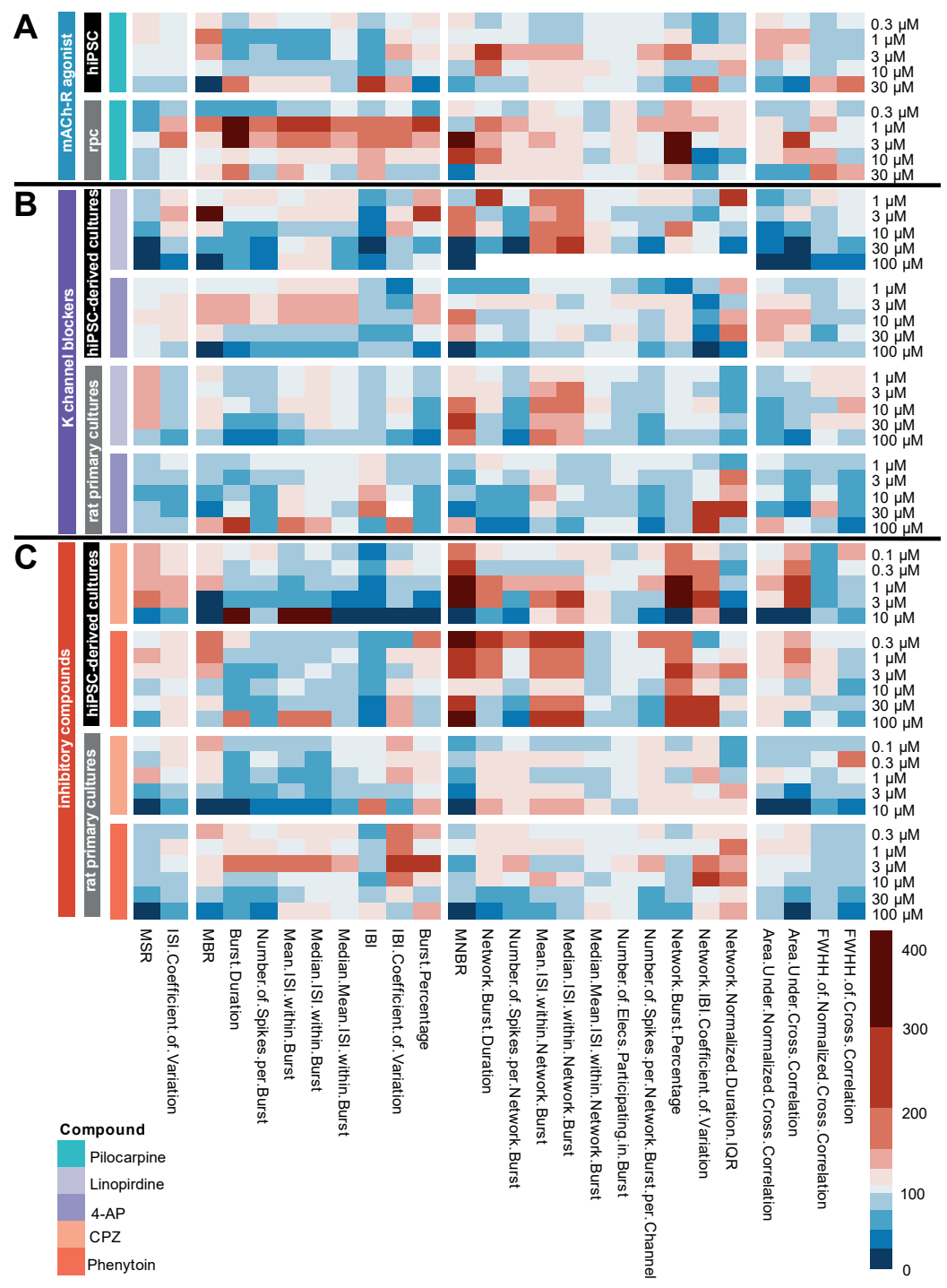


Table 3. LOECs in $\mu \mathrm{M}$ for the different compounds on different activity parameters mean spike rate (MSR), mean burst rate (MBR) and mean network burst rate (MNBR) for the human and rodent cell model. LOECs in bold illustrate the lowest LOEC in case there is a difference between the two models. - indicates no LOEC could be calculated.

\begin{tabular}{lllllll}
\hline \multicolumn{6}{l}{ hiPSC-derived neuronal co-culture } & \multicolumn{3}{l}{ Rat primary cortical culture } \\
& MSR & MBR & MNBR & MSR & MBR & MNBR \\
\hline PTZ & - & - & - & 3000 & 1000 & - \\
Amoxapine & 1 & 1 & - & 1 & 3 & - \\
Enoxacin & - & - & - & 3 & 10 & - \\
Amoxicillin & - & 3 & - & - & - & - \\
Pilocarpine & 1 & 30 & - & - & - & - \\
Linopirdine & 10 & 3 & - & - & - & 30 \\
CPZ & 1 & 10 & - & 1 & 10 & 0.1 \\
Phenytoin & 100 & - & 100 & 30 & 100 & 100 \\
\hline
\end{tabular}

\subsection{Comparison of seizure liability between the two models}

In order to compare the suitability and sensitivity for seizure liability assessment between the two models, LOECS were calculated (Table 3). For two compounds, PTZ and enoxacin, no LOECS could be calculated for exposure of hiPSC-derived neuronal co-cultures. In the case of rat primary cortical cultures, they could not be determined following exposure to amoxicillin and pilocarpine. Exposure to amoxapine results in a LOEC of $1 \mu \mathrm{M}$ on both models. In the case of linopirdine, the hiPSC-derived neuronal model is ten times more sensitive (LOEC $3 \mu \mathrm{M}$ ) than the rat primary cortical cultures (LOEC $30 \mu \mathrm{M}$ ), whereas the rodent culture is ten times more sensitive following exposure to CPZ (LOEC $0.1 \mu \mathrm{M}$ for rodent culture and $1 \mu \mathrm{M}$ for hiPSC-derived culture). However, following exposure to phenytoin these rodent cells are more sensitive (LOEC $30 \mu \mathrm{M}$ ) than the hiPSC-derived neuronal co-cultures (LOEC $100 \mu \mathrm{M}$ ). 

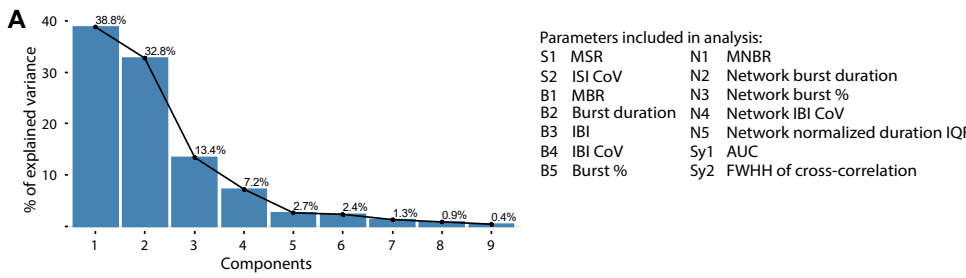

B 15 .

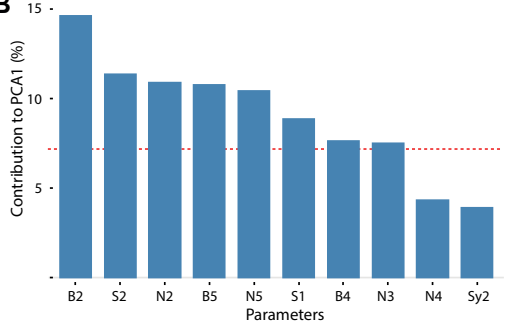

C

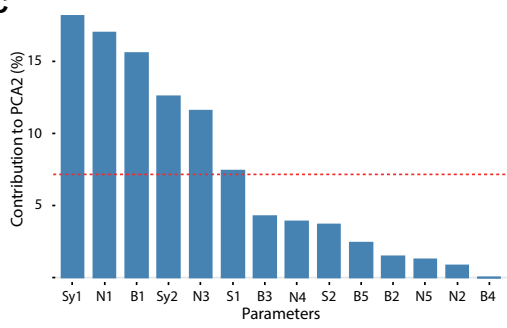

D

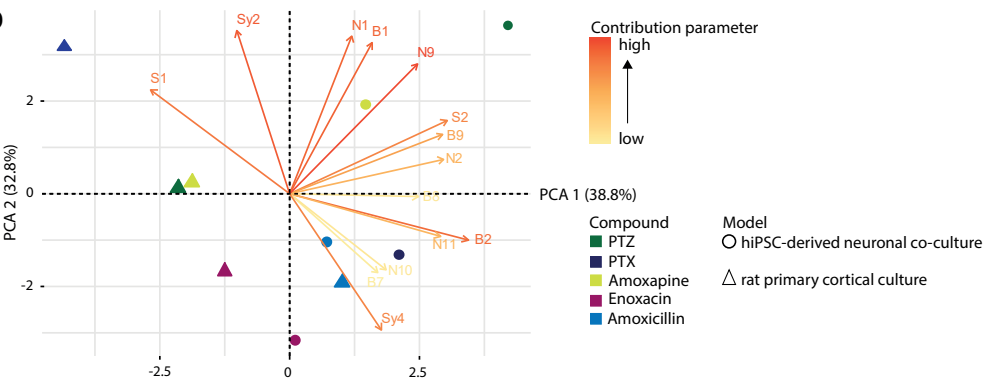

Figure 8. Scree plot displaying the percentage of variety explained by each component (A) for different $\mathrm{GABA}_{\mathrm{A}}-\mathrm{R}$ antagonists. Contribution of each parameter to $\mathrm{PCAl}(\mathrm{B})$ and $\mathrm{PCA2}(\mathrm{C})$. The red line indicates the expected contribution (7\%). Bars above the red line indicate importance for the component. Biplot depicting the contribution of parameters to the two components in arrows, darker arrows indicate higher contribution. The individual compounds are plotted in circles (hiPSC-derived neuronal co-culture) and triangles (rat primary cortical cultures) visualising a clear segregation between the two models (D). Amount of variation explained by a component is indicated in parenthesis. 


\section{DISCUSSION}

The aim of this study was to assess the suitability of hiPSC-derived neuronal cocultures for seizure liability assessment using MEA-recordings. We compared data obtained with human neuronal co-cultures with data obtained in parallel from rat primary cortical cultures.

Our MEA recordings (Fig. 1-5) show that this type of experiment is suitable for the detection of changes in network activity following modulation by different drugs. Parameters that are important for in vitro seizure liability assessment include parameters that describe the activity and the organization of the activity (Bradley et al., 2018). Examples of these parameters are spike, and (network) burst rate, but also the percentage of spikes that is incorporated in (network) bursts as well as parameters related to synchronicity. Importance of these parameters becomes clear from the heatmaps (Fig. 6-7) and PCA (Fig. 8). If in vitro seizures are defined by changes in these aforementioned parameters, then our data confirm that both models are able to detect in vitro seizures. With the exception of PTZ and enoxacin, all compounds caused changes in activity in hiPSC-derived co-cultures. No changes were detected following exposure to amoxicillin and pilocarpine in the rodent culture. However, sensitivity for the different test compounds differs between the human and rodent model in the case of linopirdine and phenytoin. (Table 3). Our heatmaps indicate that it is possible to create fingerprints and group compounds with comparable mechanisms of action (Fig. 6-7). From these heatmaps it becomes clear that burst and network burst parameters are more sensitive for excitatory changes in hiPSC-derived co-cultures than in rat primary cortical cultures. Spike parameters are more affected in rodent primary cultures than in hiPSC-derived co-cultures.

Looking at just PTZ, a compound used to induce seizures in laboratory rodents (Klioueva et al., 2001; Singh et al., 2019) and thus a known seizurogenic compound, activity significantly increased in the rodent model at concentrations comparable to concentrations reported in literature (Fig 1A; Bradley et al., 2018; Kreir et al., 2018). In the hiPSC-derived neuronal model, activity increased already at the lowest concentration tested, though not significantly. The observed trend of increased bursting after PTZ exposure on hiPSC-derived co-cultures, followed by a decrease at higher concentrations, is comparable with previous studies (Odawara et al., 2018). The cyclic anti-depressant amoxapine is known cause seizures as adverse drug reaction (Litovitz and Troutman, 1983; Kumlien and 
Lundberg, 2010). Not surprisingly, amoxapine significantly increased activity in both models (Fig. 1B). Increase in network activity was only significant in the hiPSC-derived neuronal co-culture. However, in both models, activity decreased to a minimum at the highest concentration tested. A previous study reported no significant change in spike rate following exposure of rat primary cortical cultures to amoxapine (Kreir et al., 2018), nor did this research found seizures in vivo when rats were dosed intravenously, indicating a potential inter-species difference in amoxapine sensitivity.

The fluoroquinolone antibiotic enoxacin is known to cause seizures as adverse drug reaction (Simpson and Brodie, 1985). No significant effects in the hiPSCderived model following enoxacin exposure were observed (Fig. 2A). In the rodent model, spike and burst rate did significantly increase. However, in rat hippocampal slices, seizures were found at $30 \mu \mathrm{M}$ (Gao et al., 2017). This is above our highest test concentration. Exposure to the penicillin antibiotic amoxicillin had a more profound effect on hiPSC-derived co-cultures as compared to rat primary cortical cultures (Fig. 2B), with a significant increase on burst rate. Lack of effect on rat primary cortical cultures following amoxicillin exposure is in line with previous reports (McConnell et al., 2012). However, amoxicillin has been reported to cause seizures as an adverse drug reaction in humans (Raposo et al., 2016). This thus matches our hiPSC-derived neuronal data.

When looking at the $\mathrm{GABA}_{\mathrm{A}}$ receptor antagonists (PTZ, PTX, amoxapine, enoxacin and amoxicillin) as a group in the heatmap (Fig. 6) it can be observed that all increased the overall activity in both models, with the exception of enoxacin on the hiPSC-derived neuronal co-culture. However, burst duration did not increase following exposure of rat primary cortical cultures. This in contrast to hiPSC-derived co-cultures, where an increase in burst duration was frequently observed. The hiPSC-derived co-cultures thus follow pattern one. Bradley et al. (2018) named amoxapine as a compound following pattern two. However, that was based on an exposure of $5 \mu \mathrm{M}$. Our data indicate that activity and network synchronicity decreased between 3 and $10 \mu \mathrm{M}$ on both models following amoxapine exposure. At this point, seizurogenic pattern two can be observed (Fig. 6), indicating that at low test concentrations amoxapine follows pattern one and then at higher concentrations pattern two. Both antibiotics, enoxacin and amoxicillin, are $\mathrm{GABA}_{\mathrm{A}}$ receptor antagonists and follow pattern one in both models. However, in both models and with both compounds, the observed effect is not as strong as with the other $\mathrm{GABA}_{\mathrm{A}}$ receptor antagonists. Overall, 
the increased network organization and synchronicity are more pronounced following exposure of hiPSC-derived co-cultures as compared to rat primary cortical cultures. The differences between the human and rodent model following exposure to $\mathrm{GABA}_{\mathrm{A}}$ receptor antagonists were confirmed by PCA (Fig. 8). This analysis efficiently identified differences between the two models and thus emphasizes inter-species differences. In this study, PCA has been performed on one set of compounds with a similar mode of action. However, this type of analysis holds great potential for further identifying differences and similarities between models following exposure to groups of compounds with other modes of action. Strychnine has been shown to follow pattern two. In our data, exposure of hiPSC-derived co-cultures to strychnine clearly results in pattern two (Fig. 6). In the case of rodent primary cortical cultures activity increases. However, network organization decreases in line with pattern two, as reflected in the decreased ISI $\mathrm{CoV}$, burst duration and burst percentage.

Pilocarpine is used to model epilepsy in vivo in rodents (Marchi et al., 2007). At low test concentrations pilocarpine increased activity of hiPSC-derived neuronal co-cultures, but at the highest test concentration a decrease was observed (Fig. $3 A-B)$. This biphasic effect with a strong decrease in activity following exposure to high concentrations has been reported for other compounds such as endosulfan, lindane and fipronil (Wallace et al., 2015; Dingemans et al., 2016). It could also be the result of receptor desensitization. The strong decrease could also be the result of a non-specific effect on ion channels, such as inhibition of voltage-gated calcium channels (Heusinkveld et al., 2010). Rat primary cortical cultures were not affected following pilocarpine exposure. This is in contrast to other studies that reported seizurogenic effects on rodent primary cultures and hippocampal slices following exposure to comparable concentrations (Nagao et al., 1996; Kreir et al., 2018). The data presented here do not allow for a clear indication that pilocarpine follows either seizurogenicity pattern. This problem was also mentioned by Bradley et al. (2018).

In both tested models, linopirdine decreased activity (Fig. 4) at concentrations in line with previous reports (Bradley et al., 2018). Exposure of rat primary cortical cultures to linopridine $(60 \mu \mathrm{M})$ has been shown to result in seizurogenicity pattern two (Bradley et al., 2018). Our data showed that in both models exposure to linopirdine at a comparable concentration resembles pattern two (Fig. 7). The other potassium channel blocker shown (Fig. 7), 4-AP, should resemble pattern one (Bradley et al., 2018). However, this is not clear from our data. 
CPZ significantly increased spiking activity in hiPSC-derived neuronal cocultures and rat primary cortical cultures (Fig. 5A). At higher test concentrations activity decreased. Bursting and network bursting decreased following exposure to this compound. These findings are in line with literature (Kreir et al., 2018). In rat hippocampal slices, CPZ has been shown to induce seizures at $1-10 \mu \mathrm{M}$ (Easter et al., 2007), in line with our data. Exposure of hiPSC-derived co-cultures to $\mathrm{CPZ}$ results in pattern one. However, this pattern cannot be observed in rat primary cortical cultures.

Phenytoin is designed as an antiepileptic drug and therefore expected to decrease network activity. Phenytoin (Fig. 5B) significantly decreases activity at the highest test concentration in hiPSC-derived co-cultures, at similar concentrations as others found with hiPSC-cultures (Odawara et al., 2018). Rat primary cortical cultures were more sensitive for this compound. Pattern one can be observed when hiPSC-derived co-cultures are exposed to phenytoin, whereas this is not clear in rat primary cortical cultures (Fig. 7).

We have shown that hiPSC-derived co-cultures can be used already as an initial screen for seizurogenic activity in vitro. Our data indicates differences between the human and rodent model, highlighting the need to move towards human iPSC-derived neuronal co-culture models for more predictive in vitro seizure liability assessment. However, these cells must be further characterized and validated using a larger set of test compounds before they can replace the current gold standard of rodent primary cortical cultures. 


\section{CONFLICT OF INTEREST}

The authors declare no conflict of interest.

\section{FUNDING}

This work was funded by a grant from the National Centre for the Replacement, Refinement and Reduction of Animals in Research (NC3Rs; project number 50308-372160) and by the Faculty of Veterinary Medicine (Utrecht University, The Netherlands).

\section{ACKNOWLEDGEMENTS}

We gratefully acknowledge members of the Neurotoxicology Research Group for helpful discussions. 


\section{SUPPLEMENTAL DATA}

To test if the observed neurotoxic effects are not related to cytotoxicity, cell viability was investigated with an Alamar blue, CFDA and Neutral Red assay. These assays investigate mitochondrial activity, membrane integrity and lysosomal integrity respectively.

Rat primary cortical cells were plated in transparant 96-wells plates and cultured as described in section 2.2, but at DIV4 phenol-red free medium was used. At the same day that MEA exposure took place, cells were exposed in phenol red free medium for $24 \mathrm{~h}$ at $37^{\circ} \mathrm{C}$ and $5 \% \mathrm{CO}_{2}$. Following exposure, cells were incubated with $12 \mu \mathrm{M}$ Alamar blue solution and $4 \mu \mathrm{M}$ CFDA-AM for $30 \mathrm{~min}$ at $37^{\circ} \mathrm{C}$ and $5 \% \mathrm{CO}_{2}$. Then, as a measure of cell viability, fluorescence of resorfun (product Alamar blue) and hydrolyzed CFDA were measured respectively at 530/590 nm and 493/541 nm (excitation/ emission) using a Tecan Infinite M1000 plate reader (Tecan Group LtD; Männendorf, Switzerland).

Subsequently to the combined Alamar blue - CFDA-AM assay, a Neutral Red assay was performed. One row of cells was lysed using sodium dodecyl sulfate (SDS) to be able to correct for background values. Following $20 \mathrm{~min}$, SDS and medium were removed and all wells were incubated for $1 \mathrm{~h}$ with $12 \mu \mathrm{M}$ Neutral Red solution at $37{ }^{\circ} \mathrm{C}$ and $5 \% \mathrm{CO}_{2}$. Then all cells were lysed with Neutral Red lysis buffer ( $1 \%$ glacial acetic Acid, $49 \% \mathrm{H}_{2} \mathrm{O}$ and $50 \%$ ethanol) after which plates were placed in the dark on a shaker for $45 \mathrm{~min}$. Hereafter, fluorescence was measured, again with a Tecan Infinite M1000 plate reader, at 530/ 645 (excitation/ emission). 

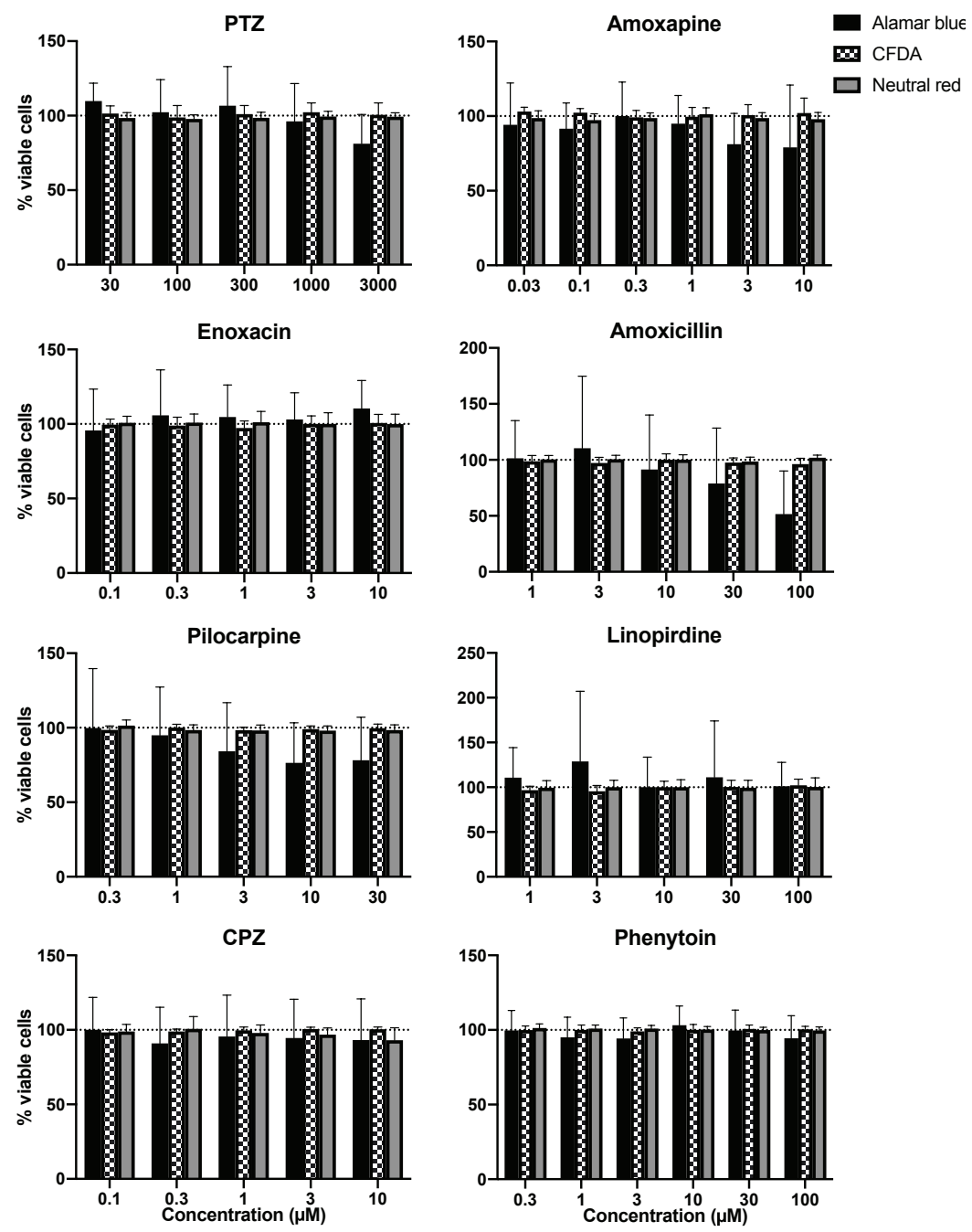

Figure S1. Cytotoxicity data of PTZ, amoxapine, enoxacin, amoxicillin, pilocarpine, linopirdine, $\mathrm{CPZ}$ and phenytoin from an alamar blue (black), CFDA (squared) and neutral red (grey) assay measured $24 \mathrm{~h}$ after a $30 \mathrm{~min}$ exposure of rat primary cortical cultures. No significant cytotoxicity can be observed. $n=15-18$ wells, $\mathrm{N}=3$ plates. Data is presented as mean $\pm \mathrm{SD}$. 


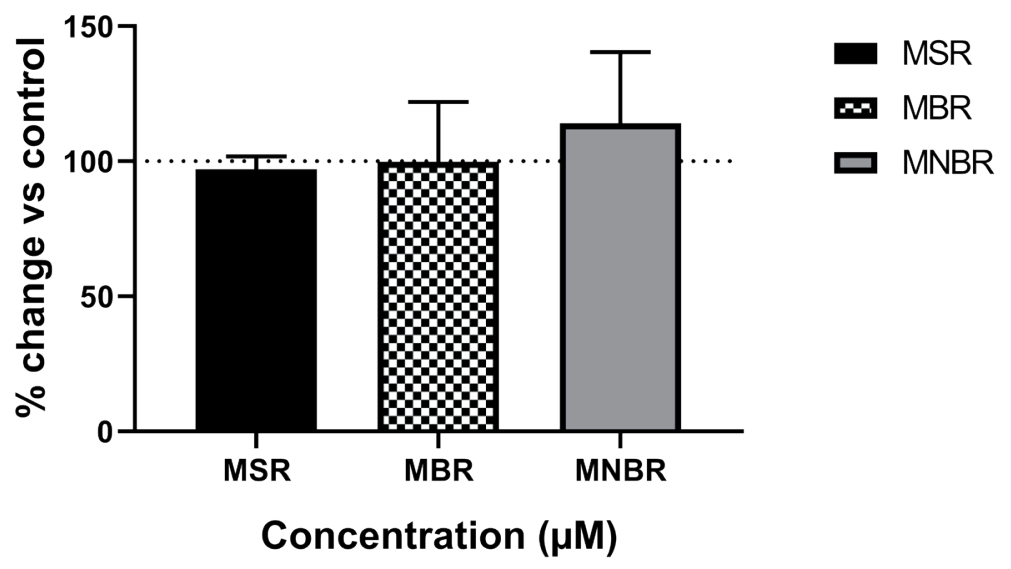

Figure S2. Acute exposure to $100 \mu \mathrm{M}$ acetaminophen did not affect mean spike rate (MSR;black), mean burst rate (MBR; blocked) or mean network burst rate (MNBR; grey) of hiPSC-derived neuronal co-cultures. $n=9-12$ wells, $N=3$ plates. Effects are depicted as average $\%$ change of control (solvent control set to $100 \%$; dashed line) \pm SEM. 


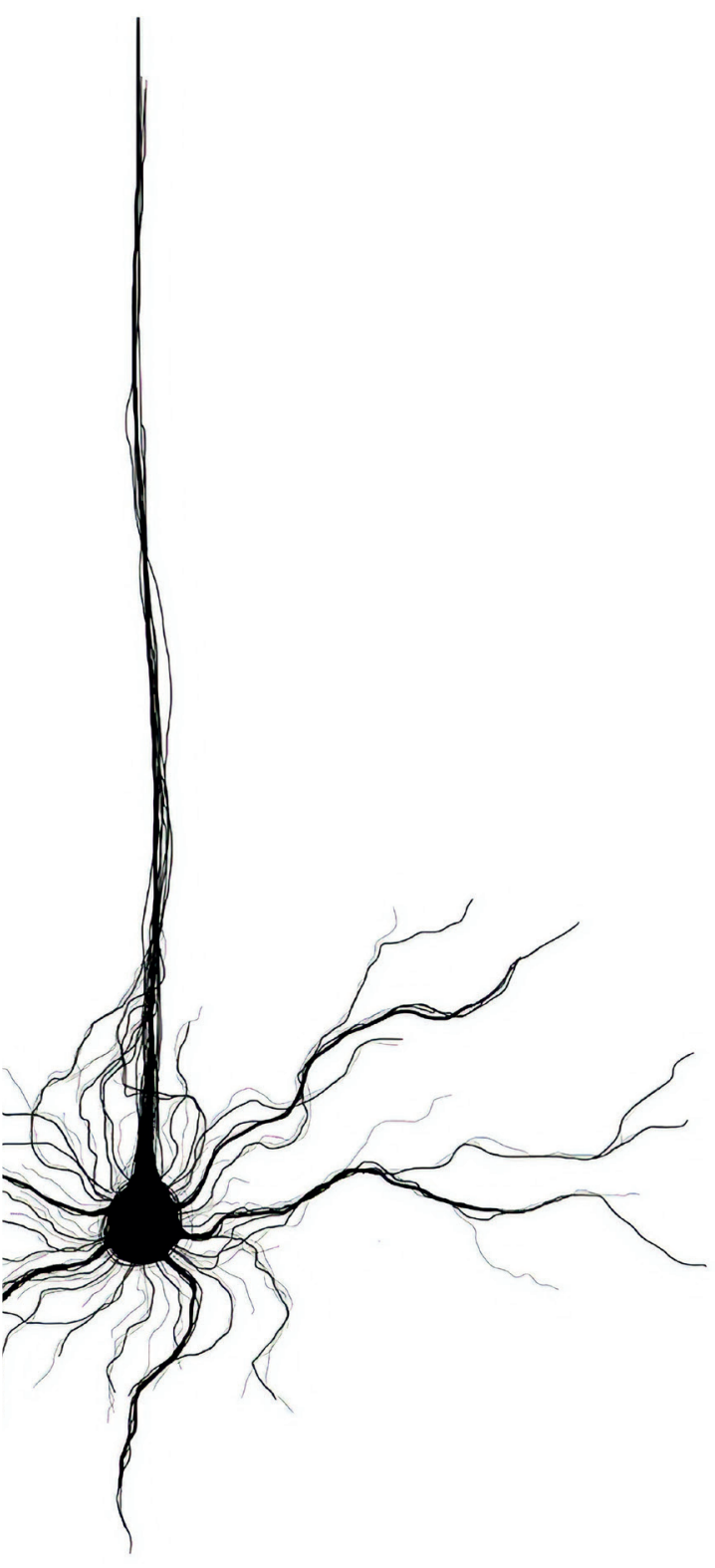


Part V

SUMMARY, GENERAL

DISCUSSION AND

CONCLUSIONS 
Chapter 9

Summary, General Discussion and Conclusions 
Due to the increase in patients with mental illnesses or neurodegenerative diseases, there is an increasing demand for drugs targeting the central nervous system. Because such drugs have a higher brain penetration and a higher affinity for targets in the central nervous system, they are also more prone to cause seizures as an adverse drug reaction. Reliable detection of compoundinduced seizures is challenging because the central nervous system (CNS) is highly complex with a high degree of integrated neuro-cellular processes. Due to regulations, current models used to detect the potential of drugs to cause seizures are either in vivo or ex vivo models, thereby relying strongly on animals. The current strategies use a large amount of test animals, making testing labour intensive, expensive and time consuming. Moreover, using test animals is ethically debated and the outcome not always predictive for human risk. Successful and cost-effective replacement of these in vivo and ex vivo models will drastically reduce the numbers of animals used for drug-development. Therefore, there is a clear need for alternative approaches. Recently, hiPSC-derived neurons have become commercially available, offering an opportunity for new test strategies. In this thesis, the applicability of human neuronal models based on commercially available iPSCs for in vitro seizure liability and neurotoxicity testing was assessed. The main questions asked whilst developing these models were:

- Does the model form a neuronal network resembling the in vivo complexity?

- Does the network become spontaneously active exhibiting synchronised bursting behaviour?

- Does the model exhibit seizure-like activity following exposure to a seizurogenic compound?

- How does the model compare to the current gold standard of rat primary cortical cultures?

\subsection{Test strategies for in vitro seizure liability assessment}

In order to answer the first question, a number of techniques needed to be established to verify the formation of active networks. The first starting point to confirm the presence of networks and thus answering question one is immunocytochemistry. Staining with antibodies allows for visualisation of different cell types present in the networks and thereby the networks themselves as outlined in chapter 3. Also, the state of maturation can be confirmed. The presence of neurotransmitters or direct effects of toxicants on ion channels or neurotransmitter receptors can be assessed with targeted approaches such as single-cell calcium imaging (chapter 5). This technique also allows for the 
assessment of the presence of active networks as outlined in chapter 3 and thus provides an answer to question two. A more high-throughput technique for assessment of network activity involves MEA recordings (chapter 3). The benefit of using the MEA technique is that it allows for the study of neuronal network activity. This process reflects integrated endpoints. Thus, in contrast to the voltage-clamp technique which provides information on the effect of a compound on a single receptor (chapter 2 and 4), MEA recordings reflect the physiologically-relevant effects on the full network (McConnell et al., 2012; Nicolas et al., 2014; Valdivia et al., 2014). Also, since it is non-invasive, it allows for repeated measurements over time in the same plate, allowing the study of network development. Besides studying network activity and bursting behaviour, the MEA technique allows for modulation of neuronal networks of the hiPSC-derived model and the rat primary cortical model with toxicants such as for example PFOS and PFOA (chapter 4). This technique thus aid in answering question three. Application of the techniques outlined here demonstrated that they can be used to study network formation and activity as well as network modulation (chapter 2-4).

\subsection{Human iPSC-derived models for in vitro seizure liability assessment}

At the start of this research project, there was no well characterised commercially available hiPSC-derived model for in vitro seizure liability assessment and neurotoxicity testing, nor was there knowledge on how these neurons compare to the current gold standard of rat primary cortical cultures. Therefore, we started with obtaining different hiPSC-derived neurons from different suppliers that existed at that time.

As shown in chapter 5 , mono-cultures of three different commercially available hiPSC-derived neurons form complex neuronal networks with excitatory and inhibitory neurons. These mono-cultures thus contain different types of neurons, such as GABAergic and glutamatergic neurons, but no supporting cells. Using single cell calcium imaging, we showed the presence of functional neurotransmitter receptors (GABA, glutamate and acetylcholine) and VGCCs on the membranes of these neurons at early culture stage. These mono-cultures also develop spontaneous network activity as shown using MEA recordings. However, this activity is relatively low level when compared to rat primary cortical cultures. Also, (network) bursting behaviour is (almost) completely absent. Even though activity is at a different level than rat primary cortical cultures, human cultures behave comparably following pharmacological and physiological modulation, 
indicating their potential for neurotoxicity testing. These data thus answer question one and two. The three different hiPSC-derived mono-cultures develop complex networks with spontaneous network activity. When comparing the activity of these mono-cultures with rodent primary cortical cultures (question four), activity is lower. However, addition of astrocytes to neuronal mono-cultures may enhance synaptic maturation and transmission (Clarke and Barres, 2013) and increase firing frequency and bursting behaviour (Tang et al., 2013; Odawara et al., 2014; Ishii et al., 2017). Astrocytes may also influence the sensitivity of cultures towards toxicological insults (Takemoto et al., 2017). This is especially important when it comes to excitotoxicity, an event that is associated with the development of seizures. Besides astrocytes, the ratio of excitatory glutamatergic to inhibitory GABAergic neurons can greatly influence behaviour of neuronal cultures. We therefore developed co-cultures with and without hiPSC-derived astrocytes and containing various ratios of glutamatergic to GABAergic neurons. In this next step, the best performing neuronal co-cultures were chosen for further research.

To further answer the question concerning network formation, the different cocultures with varying ratios of glutamatergic neurons and GABAergic neurons in presence or absence of astrocytes developed more complex networks than mono-cultures as demonstrated in chapter 6 . In order to further answer question two, developmental curves to investigate the development of activity of the cocultures were made. Co-cultures with astrocytes, i.e. one model with addition of extra GABAergic neurons ( $30 \%$ of total neurons) and one without ( $10 \%$ of total neurons), developed more synchronised networks than mono-cultures. In contrast to mono-cultures, the co-cultures containing astrocytes developed pronounced bursting behaviour. Also, network bursting activity is higher in cultures containing astrocytes. This is in line with the notion that adding astrocytes would aid network synchronisation and thereby enhances bursting and network bursting. These co-cultures with astrocytes exhibit a pattern of development that is comparable to rat primary cortical cultures with increased spiking and (network) bursting activity over time and a higher number of spikes occurring in (network) bursts. The co-culture containing extra GABAergic neurons exhibited, logically, a lower activity. The two co-cultures with astrocytes were selected for a primary neurotoxicity assessment. Since the ultimate model must be able to detect seizures, two compounds that are known to increase neuronal activity in rodent primary cortical cultures: PTX (Bradley et al., 2018; Kreir et al., 2018) and endosulfan (Dingemans et al., 2016) were used. Data obtained from this study helped answering question three, whether or not the activity of the model 
increased following exposure to seizurogenic compounds. We found that there was a difference in chemical sensitivity and the manner in which the different models reacted to chemical insults. In the culture without extra added GABAergic neurons, activity did not increase following exposure. Lack of effect could be due to the sparsity of GABAergic receptors (targets for the compounds), the already high level of activity in cultures of mainly excitatory glutamatergic neurons, or a combination of both. The fraction of inhibitory neurons in the network and/ or the degree of excitability present in the network may thus be critical for the chemosensitivity of these models for seizurogenic compounds and GABA receptor antagonists. When both models were exposed to $\mathrm{MeHg}$, a compound that decreases activity in rat primary cortical cultures (Dingemans et al., 2016), the culture without extra GABAergic neurons was more responsive. It thus seems that a culture with a high activity is more sensitive towards neurotoxicants that decrease neuronal activity. We now thus showed that astrocytes greatly influence level and pattern of neuronal activity. They are needed to synchronise activity and to enhance (network) bursting behaviour. However, too many astrocytes will suppress activity and cause cell clustering. This cell clustering significantly hampers MEA analysis. It is thus critical to find an optimum balance between level of synchronisation and cell clustering. The hiPSC-derived neuronal co-cultures used in this model contain $50 \%$ astrocytes. It does not seem recommendable to use a higher percentage. This study also indicates that the sensitivity of a model towards a toxicant depends on the ratio of GABAergic to glutamatergic neurons. For seizure liability assessment, presence of GABAergic neurons in a percentage higher than $10 \%$ thus seems to be important.

After establishing the importance of astrocytes as well as the ratio of inhibitory to excitatory neurons, we obtained hiPSC-derived neurons and astrocytes from three different commercial suppliers and created co-cultures (chapter 7). The applicability of these models for in vitro seizure liability was assessed. The three different hiPSC-derived neuronal co-cultures formed complex neuronal networks that are comparable with rat primary cortical cultures (Hondebrink et al., 2016). As the human models were cultured according to manufacturers' protocols, they differed in seeding density, ratio of neurons to astrocytes and ratio GABAergic to glutamatergic neurons. Nevertheless, all three models developed neuronal activity with bursting and network bursting over time. However, models with higher seeding densities exhibit higher network activity. As said earlier, activity and synchronisation are also enhanced by astrocytes. Not surprisingly, the model with the highest percentage of astrocytes present is the model with highest level 
of synchronisation. Although the percentage of astrocytes in this model was lower than the $50 \%$ of the models in chapter 6 , activity was higher. Despite the differences, all three models developed neuronal activity in a manner comparable to rat primary cortical cultures (Brown et al., 2016). The next step was to assess whether these models are suitable for in vitro seizure liability assessment and whether they perform comparable to or better than rat primary cortical cultures, thereby thus focussing on questions three and four. To that aim, hiPSC-derived models and rat primary cortical cultures were exposed to known-seizurogenic compounds PTX, 4-AP and strychnine. Data from this study indicate that all hiPSC-derived models have potential for in vitro seizure liability assessment because all three tested compounds affected activity and synchronicity. Even more interesting, the hiPSC-derived neuronal co-cultures detected seizures at the same or even a lower level or than rat primary cortical cultures. An important finding here is that when using MEA recordings for seizure liability assessment, multiple parameters must be studied and not just activity parameters. Seizures affect networks in a multitude of ways, therefore more parameters describing the state of the network must be taken into account. Also, a one to one comparison of hiPSC-derived neuronal co-culture data to rat primary cortical data is difficult. Differences between humans and rodents exist and in turn can result in different sensitivities and hits.

The previous study (chapter 7) was performed with a small number of compounds. We therefore selected the model with the shortest culture duration and a high network activity for further testing as described in chapter 8. This study mainly focusses on questions three, the suitability of the model for seizure liability assessment and four, a comparison of hiPSC-derived neuronal co-culture data with rat primary cortical cultures. In this study, nine additional known (non-) seizurogenic compounds (PTZ, amoxapine, enoxacin, amoxicillin, pilocarpine, linopirdine, $\mathrm{CPZ}$, phenytoin and acetaminophen) were chosen to further test on one selected hiPSC-derived neuronal model. In parallel, rat primary cortical cultures were exposed to the same compounds and compared to the selected hiPSC-derived neuronal model. Both models reacted upon exposure to seizurogenic compounds. However, not all compounds were a hit in both models. PTZ and enoxacin could not be detected in the hiPSC-derived neuronal coculture. The rat primary cortical culture could not be modulated with amoxicillin and pilocarpine. Also, the human and rodent model behaved differently following exposure and for some compounds, the sensitivity differed. The hiPSC-derived neuronal co-culture was ten times more sensitive for linopirdine than rat primary 
cortical cultures, whereas the latter was about three times more sensitive for phenytoin. Our MEA data showed that in hiPSC-derived neuronal co-cultures (network) burst parameters are more sensitive for excitatory changes, whereas in rodent cultures, spike parameters are more affected. Because seizurogenic compounds can be detected in a way comparable to the current gold standard of rat primary cortical cultures, this study thus shows that hiPSC-derived neuronal co-cultures can already be used as a first screen in in vitro seizure liability assessment. However, this study also highlights, as in chapter 7, that there are differences between the human and rodent model. These differences are again highlighted in the principal component analysis that showed that the different parameters are affected differently depending on the model. Although hiPSCderived neuronal co-cultures are able to detect seizurogenic compounds, it is too early to state that they can replace rat cortical cultures as the current gold standard, as becomes clear in the following section.

\subsection{Risk assessment and hiPSC-derived neurons}

The research presented in this thesis shows that hiPSC-derived neuronal cocultures can be used as a first screen in neurotoxicity testing and seizure liability assessment. However, in order to move towards animal-free neurotoxicity testing, the predictive value of results obtained with hiPSC-derived neuronal cocultures must be elucidated. Consequently, a connection with in vivo outcomes must be made. However, in vitro exposures often do not match one to one with in vivo exposures. This is because in the in vitro situation, exposure is directly to the neuronal networks, in contrast to the (human) in vivo situation, where processes of absorption, distribution, metabolism and excretion (ADME) are present. These ADME processes can alter the actual target concentration compared to the (e.g., orally) exposed concentration. Thus, an in vitro hit concentration is not necessarily a concentration that causes adverse drug reactions or health concerns in real life. To translate the laboratory (in vitro) setting to the real in vivo world, an in vitro to in vivo extrapolation (IVIVE) must be performed. Extrapolation of in vitro to in vivo results can be based on physiologically-based pharmacokinetic (PBPK) models (Bal-Price et al., 2008; Krewski et al., 2010). However, for these models to function properly, values for parameters such as body weight, brain volume, brain to blood partition coefficient and liver to blood partition coefficient must be used as input to the model (Croom et al., 2015). These values can be derived from either intoxication or pharmacovigilance case reports or, if no human data is available, animal experiments. 
In this study, marketed drugs that are known to cause seizures as adverse drug reaction (amoxapine, enoxacin, amoxicillin, pilocarpine, CPZ and linopirdine) as well as known seizurogenic compounds (PTZ, PTX, strychnine and 4-AP) were tested (chapter 7-8). The marketed drugs have gone through thorough testing during the drug development pipeline by the pharmaceutical company before they could enter the market. Therefore, a broad range of data must be available from pharmaceutical companies that could be used as input for PBPK models. The outcome of the PBPK model will provide information about the predictive value of hiPSC-derived models for seizure liability and neurotoxicity. Unfortunately, until today this data is not easily accessible for researchers, needlessly hampering validation of hiPSC-models. This also restricts replacement of in vivo studies with in vitro studies. Future efforts should thus be made to make data from approved drugs publicly (or at least for research purposes) available. Both academia and industry could benefit from such a cooperative environment.

The challenge on the in vitro side for PBPK modelling and risk assessment in general is to decide which parameter of the MEA-analysis to include. MEArecordings yield a wealth of data that can be translated in many metric parameters. Till today there is no consensus on what the most predictive MEA parameter is. As became clear from chapter 7-8, classic activity parameters such as spike rate and (network) burst rate do not always reflect changes occurring in the network following exposure to seizurogenic compounds. Other parameters such as burst duration, burst percentage or synchronicity related metrics might be more informative. On top of that, in vitro $\mathrm{EC}_{50}$ values are frequently used for modelling approaches (Croom et al., 2015; Sharma et al., 2017). This is problematic with seizurogenicity data where there often is an increase in activity followed by a decrease, probably as a result of overexcitation of the network. The shape of the resulting curve hampers proper $\mathrm{EC}_{50}$ calculations. Therefore, a threshold approach could be used, meaning that the LOEC or NOEC might be more suitable. A challenging factor here is that value of the NOEC, LOEC and $\mathrm{EC}_{50}$ depends on the MEA parameter chosen. One approach could be to use the most sensitive parameter and to decide this on compound to compound basis.

\subsection{Human iPSC culture conditions and neuronal differentiation}

For risk assessment, regulatory purposes and research in general, it is of uttermost importance to obtain reliable and reproducible results. Generation of hiPSCs is a relatively new technique (Takahashi and Yamanaka, 2006), as well as the differentiation steps that follow. Many researchers and companies now 
start to produce hiPSC-derived neurons, under slightly different conditions with slightly different protocols. Those on first sight small differences, can have a big impact on the resulting batch of hiPSC-derived neurons, thus potentially hampering reproducibility.

\subsubsection{Handling and maintenance hiPSCs}

Maintenance of hiPSCs requires technical skills since the undifferentiated state is instable and cells are sensitive for mechanical stress (Konagaya et al., 2015). Also, slight changes in culture and growth conditions can result in unwanted differentiation (Little et al., 2019), thereby creating a batch of cells that is different from the expected ones. A study in which the same iPSC line was tested across five different laboratories detected poor cross-site reproducibility and identified the laboratory as the largest source of variation (Volpato et al., 2018). However, with advances in technology such as automated robots for culturing (Konagaya et al., 2015) and culture media with growth factors that can maintain growth for multiple days reducing the number of handling moments can help reduce this issue. Besides enhancing reproducibility, automation has as additional benefit that it strongly reduces labour costs and intensity (Jenkins et al., 2016). In the case of commercially available hiPSC-derived neurons, researchers do not have to differentiate the cells themselves, saving a lot of time. Also these cells come in large quality controlled batches (Little et al., 2019), ensuring that researchers can perform a complete study on the same cells.

\subsubsection{Medium composition}

Commercial suppliers often provide cells in full packages with supplements and sometimes even basal medium. Though this strongly enhances the ease of use of commercially available hiPSC-derived neuronal cells, it makes comparison between models difficult. As shown in chapter 8 , all three hiPSC-derived neuronal models developed spontaneous neuronal network activity, but activity levels varied. This could be due to differences in seeding density, but could also be due to differences in medium composition. It has been shown that concentrations of salt and serum (or serum-replacement factors) in media directly affect neuronal network activity (Bardy et al., 2015). Chapter 8 also demonstrated that all three models could be modulated with PTX, strychnine and 4-AP. However, since these cells came from three different suppliers, they were cultured in three different media types. It has been shown that media substantially influence the effect of toxic insults on hiPSC-derived neurons (Joshi et al., 2019). It is thus hard to know whether the different outcomes are the result of different cell densities, model 
composition, receptor profile, medium composition or a combination. Especially now multiple companies produce hiPSC-derived neurons, all with their own basal media and media components, variation in culture conditions and thus possible variation in study outcomes is likely to increase.

\subsubsection{Characterisation and maturity of hiPSC-derived neurons}

For neurotoxicological purposes it is extremely meaningful to choose a model that fits the research question and mimics the in vivo situation as closely as possible, whilst keeping the model as simple as possible (Westerink, 2013). In order to do this a full model characterisation should be done elucidating at least the receptor profile. This will illustrate the targets present in the model. Unfortunately, this is a laborious process and cannot been done quickly. However, when developing hiPSC-derived neurons, it must be done. Again, commercially available hiPSCderived neurons may offer a solution. Frequently, companies have performed a thorough characterisation and should thus have a full receptor profile available.

Following the differentiation process, hiPSC-derived neurons can becryopreserved and stored without losing functionality (D'Aiuto et al., 2014; Wakeman et al., 2017) or affecting the neuronal differentiation program (Nishiyama et al., 2016). However, there is probably an optimal maturation stage at which neurons can be frozen for optimal recovery, as is the case for hiPSC-derived cardiomyocytes (Preininger et al., 2016). The behaviour of thawed hiPSC-derived neurons is thus likely to be affected by the maturation stage at which they were frozen.

\subsection{4 (Human) iPSC database}

All the factors mentioned above make it challenging to estimate what a good hiPSC-derived neuronal cell type is and which one to use to answer the research question. Often most information is available, but not easily accessible. The field would thus benefit from the development of an hiPSC database. This database should contain information on disease-relevant and healthy control hiPSCderived neuronal cell types with documented donor consent where applicable, quality control data and a full characterisation of the receptor profile (Engle et al., 2018). Furthermore, this databank should contain information on medium composition and culture protocols. Especially with the rapidly developing field of commercially available hiPSC-derived neurons, more transparency is a must. Due to the rapid developments in the field of hiPSC-derived neurons, the number of companies and cells to choose from rises, presumably lowering the costs of these still expensive cells. A downside of the rapid development is that the variety 
of media and cell types and protocols only increases, clearly indicating an urge for structure. Here, the database could be of help. On top of that, the suggested database could help enabling the acceptance of hiPSC-derived neurons for regulatory purposes as it provides a starting point for standardisation of culture and exposure protocols.

\subsection{Genetic make-up}

When using hiPSC-derived neuronal cultures for neurotoxicity testing and seizure liability assessment, it is important to realise how these cells are generated. Often hiPSC-derived neurons are clonally derived (Hong et al., 2013; Willmann et al., 2013). Also, when this is not the case, all hiPSC-derived neurons in one batch come from the same donor. This means that in either way the genetic variation is low and cells will behave comparably. It could also be that the cell or donor in question has a non-lethal spontaneous DNA mutation or has undergone genetic changes during the reprogramming process, thereby not representing the normal situation. Whereas iPSC-derived neurons obtained from one donor might be very informative, as will be described in the following section, the genotype of the donor is of paramount importance for interpretation of the results. It thus raises some questions in regards to human risk assessment for the general population. It is very unlikely that this one donor represents the general population. Consequently, it could be argued that for a proper risk estimate, a pooled sample of hiPSC-derived neurons from multiple donors must be used. A downside of pooling could be that specific effects are averaged out due to a part of the sample population being extremely sensitive and another part being insensitive. Another option would then be to create a test battery in which cells from different donors are subsequently tested and an average result can be used for risk estimates. To do this for every compound would be a very laborious process. However, the classical safety factor 10 for human-to-human variability in risk assessment might benefit from refinement. A way to circumvent laborious testing and still refine the safety factor could be to test a selection of compounds, with known in vivo effects, on the hiPSC-derived neuronal battery. Outcomes of these test can show whether the safety factor 10 is adequate. If not, a new safety factor for interindividual differences can be derived from the obtained data.

Once going down the road of improving risk assessment, there is a lot of potential for tailoring the process. It has long been known that the elderly (Nolan and O'Malley, 1988; Routledge et al., 2003) and children (Landrigan, 1999) are more vulnerable for adverse drug reactions. Therefore, a test battery in which 
subsequently hiPSC-derived neurons from the general population, elderly and children are tested could improve quality of the predictions and provide more tailor-made advice. Taking that even on step further, recent insights on sexspecific differences in drug efficacy and adverse drug reactions (Nicolson et al., 2010), indicating that women suffer more adverse drug reactions than men (Gochfeld, 2017), argue in favour of a test strategy with male and female hiPSCderived neurons tested separately. The suggested test strategies could help prevent drug attrition.

\subsection{Patient-derived hiPSCs}

Whereas the previous section concentrated on opportunities for improving risk assessment, hiPSC-derived neurons can further be used during drug development. Human iPSC-derived neurons from different disease backgrounds can help provide clues about differences in vulnerability towards certain drugs. Patient-derived hiPSC-derived neurons have already been successfully cultured from patients suffering several neurodegenerative diseases such as Huntington's (Zhang et al., 2010), Alzheimer's (Ochalek et al., 2017) or Parkinson's disease (Schöndorf et al., 2014). Pharmaceutical companies could integrate these hiPSCderived disease models in their test battery. Following successful culturing of these cells, high throughput screenings can be performed to identify promising novel compounds (Avior et al., 2016; Little et al., 2019) that alleviate or reverse disease symptoms. Besides efficacy identification and novel compound discovery, these models could later on be used for safety testing. Incorporation of patientderived cells in the drug development pipeline creates a more targeted approach and will most likely enhance the success rate of drug development. Also, it can contribute to reduction of drug attrition numbers. Additionally, the use of patient-specific hiPSC-derived neurons paves the road towards personalised medication. Implementation of these neurons in healthcare could significantly reduce adverse drug reactions because susceptibility and vulnerability of the patient may be established on forehand. Tests can be performed to deduct optimal dosing. Also, since patients frequently use several types of medication, cross reactions can be tested. Although this patient-derived work will be very labour intensive and time consuming with the currently available technology, it holds great promise for the future and enables the way to disease-specific neurotoxicity and safety testing. 


\subsection{Model improvements?}

Throughout this thesis, the developed hiPSC-derived neuronal co-cultures have been compared to the current gold standard of rat primary cortical cultures. This is certainly a good starting point to explore the possibilities of human models. Many compounds that have been tested on the MEA on rat primary cortical cultures, have also been tested in vivo, allowing for an in vitro to in vivo comparison (see for example Kreir et al., 2018), thereby confirming the predictivity of the rodent cell model. Comparing human in vitro data with rodent in vitro data will certainly aid validation of the models, but the best validation is comparing human in vitro data with human in vivo data, for example with pharmacovigilance reports. Since humans and rodents diverted 60 million years ago, their brain structures are very different (Clowry et al., 2010). It is therefore likely that their in vitro behaviour differs. Thus, instead of optimising the hiPSCderived neuronal co-cultures in a way that they resemble rat primary cortical cultures, other points of optimisation might be interesting to investigate.

The hiPSC-derived neuronal models presented in this thesis are all cultured in a two dimensional (2D) plane. A criticism of the use of $2 \mathrm{D}$ models is that they might not capture the complexity of the three dimensional (3D) in vivo brain. Due to the absence of layers, 2D models lack the hierarchical structure, connectivity and matrix environment that is found in 3D. Organoids and spheroids from hiPSC-derived neurons have been shown to capture early development of the human brain (Gabriel and Gopalakrishnan, 2017; Pamies et al., 2018; Leite et al., 2019). These models look very promising, but will be challenging to effectively couple to MEA-recordings. Up until now, MEA plates are designed in such a way that the electrodes are located at the bottom of a well. This means that they only record activity from cells in their direct (2D) vicinity. Although signals travel throughout the network, there is no guarantee that signals from the top of the well always reach the bottom of the well. For this reason, the measurement most likely does not represent full network activity. Therefore, the MEA technique is not yet suitable for measurements of $3 \mathrm{D}$ cultures. MEA plates that contain electrodes rising from the bottom of the well, thereby making it possible to also record activity at the top of the organoid or spheroid, would greatly increase the suitability of $3 \mathrm{D}$ cultures to replace $2 \mathrm{D}$ cultures for in vitro seizure liability assessment. At this moment, imaging approaches as described in chapter 3 are likely more suitable for seizure liability assessment and neurotoxicity screens in 3D cultures. Another challenging issue with $3 \mathrm{D}$ organoids or spheroids is, is that they often lack 
vasculature, preventing the inner cells from receiving oxygen and nutrients and limiting the extent to which they can grow (Sun, Ng, et al., 2018).

In real life, drugs have to cross the blood-brain barrier. Thus, to ideally mimic drug exposures in vitro, addition of a blood-brain barrier to neuronal co-cultures is favourable. Nowadays, blood-brain barriers, including the microvessels, can be cultured with hiPSC-derived cells (Linville et al., 2019). These hiPSC-derived blood-brain barriers have been incorporated with neurons and astrocytes on microfluidic chips (Vatine et al., 2019). Incorporating a blood-brain barrier in the neuronal co-culture allows for a direct assessment of the given dose on the neuronal network. If the drug is actively transported over the blood brain barrier, all of the administered compound might reach the brain. In cases where this does not happen, the exposure will be lower than the given dose. To continue the research presented in this thesis, it would be interesting to incorporate a bloodbrain barrier in the MEA well together with the astrocyte-neuron co-culture. This way, it will become clear in one assay whether a test compound passes the bloodbrain barrier and if it has an effect on the neurons. However, once it is known that the compound can pass the blood-brain barrier, addition of such a barrier might unnecessarily complicate the model.

In addition to interesting research opportunities such as adding a blood-brain barrier to the model or attempting to grow the model in 3D, an easier but maybe even more interesting topic can be studied. As mentioned in the introduction, human iPSC-derived neuroprogenitor cells can be differentiated into several types of neurons such as dopaminergic (Swistowski et al., 2010), glutamatergic (Zeng et al., 2010), cholinergic (Hu et al., 2016), serotonergic (Vadodaria et al., 2018) and GABAergic neurons (Liu and Zhang, 2011) as well as several types of supportive cells. By mixing these different types of neurons and supportive cells in the right ratios, specific brain regions of interest can be modelled. Brain regions of interest for seizure liability assessment include the neocortex, hippocampus and amygdala (Rogawski and Löscher, 2004). This is in contrast to for example Parkinson's disease where dopaminergic brain areas, such as the substantia nigra (Balestrino and Schapira, 2019), are more interesting to study. Models developed in this thesis most closely resemble the (rodent) cortex (Görtz et al., 2004; Markram et al., 2004; Tukker et al., 2016) and will thus mainly provide information on seizure liability in the cortex. It would therefore be very interesting to tweak these models in such a way that they resemble the other seizure onset regions and see how that affect our results. However, a complicating factor is that exact ratios 
of the different cell types are not always known for these regions and may differ between different life stages. For better seizure liability assessment, multiple hiPSC-derived neuronal co-cultures mimicking the different brain regions of interest might be needed.

The points mentioned above are suggestions for model improvement. That said, it really depends on the research question how complicated the model should be. If it is already known that a compound can cross the blood brain barrier, there is no need for inclusion of such a barrier in the model. Similarly, if it turns out that $2 \mathrm{D}$ models have the same predictivity as $3 \mathrm{D}$ models, there is no need to culture more complicated models. Every step taken to add complexity may increase the variability and cause problems with reproducibility.

\subsection{Concluding remarks and recommendations}

The research presented in this thesis aimed at developing an hiPSC-derived neuronal model for in vitro seizure liability assessment. In order to assess the suitability of the developed model, four main questions related to network formation, network activity, seizure liability and comparison with the current gold standard were main themes in this thesis. The data presented show that hiPSC-derived neurons develop spontaneously active networks that exhibit (network) bursting. Addition of astrocytes enhances activity and network synchronisation. In addition, the level and pattern of activity is greatly influenced by the ratio of glutamatergic to GABAergic neurons. Also, the way networks react to exposure to different known (non-)seizurogenic compounds is greatly influenced by the composition of the network. The selected hiPSC model could be modulated with known seizurogenic compounds such as for example PTZ, PTX and amoxapine and with non-seizurogenic compounds such as phenytoin and CPZ. This thus answers questions one, two and three. Human iPSC-derived neuronal models form complex in vitro networks resembling in vivo complexity (question one), whilst developing spontaneous network activity and (network) bursting behaviour (question two) that can be modulated with seizurogenic compounds (question three). However, answering question four is more difficult. In some case the human model was equally sensitive as rodent primary cortical cultures, whereas in other cases sensitivity was different. However, this is most likely a logical result of the differences between humans and rodents. As a one to one comparison of the data is not yet possible, the question now is, which model is more predictive for human seizure liability? Is that an in vivo rodent model with an intact physiology and metabolism, an ex vivo rodent model or is it an in 
vitro human model that circumvents inter-species differences? If in vitro models are used, do they need to be in 3D? Must a blood brain barrier be incorporated? Do we need to move towards human-on-a-chip models? Though this thesis does not answer the previous questions, the data presented here indicate that hiPSCderived neuronal co-cultures perform just as well or even outperform the current gold standard of rat primary cortical cultures when it comes to in vitro seizure liability assessment. Therefore, hiPSC-derived neurons can trigger a paradigm shift in in vitro neurotoxicological research and seizure liability assessment towards animal-free testing in the future.

To sum up the main findings and recommendations of this thesis:

- Human iPSC-derived neuronal co-cultures can be used as a first screening tool for in vitro neurotoxicity testing and seizure liability assessment

- Thorough characterisation of new hiPSC-derived neuronal models is critical prior to incorporating them in in vitro seizure liability assessment strategies

- It is important to choose a model that resembles the brain region of interest, i.e. with a ratio of glutamatergic to GABAergic neurons and neurons to astrocytes that mimics the in vivo brain area

- To better map the complexity of the brain, different models mimicking different brain regions of interest might be needed

- Astrocytes play a critical role in (network) bursting behaviour and synchronicity

- The ratio of glutamatergic to GABAergic neurons and the ratio neurons to astrocytes strongly influences sensitivity towards toxicological modulation

- Human iPSC-derived neuronal co-cultures perform comparable or, in some cases, better than rodent primary cortical cultures in in vitro seizure liability assessment

- A database with information on hiPSC-derived neurons can aid standardisation and trigger acceptance by regulatory authorities

- Transparency of pharmacological companies will aid validation of hiPSCderived neuronal models

- Human iPSC-derived neurons offer great opportunities for improvement of risk assessment

- A suitable hiPSC-derived neuronal co-culture for in vitro seizure liability assessment does not necessarily have to behave the same as the current gold standard of rat primary cortical cultures 
APPENDICES 


\section{REFERENCES}

Abud, E. M., Ramirez, R. N., Martinez, E. S. et al. (2017). iPSC-Derived Human Microglialike Cells to Study Neurological Diseases. Neuron 94, 278-293.e9. https://doi.org/10.1016/j. neuron.2017.03.042.

Alachkar, A., Łażewska, D., Latacz, G. et al. (2018). Studies on Anticonvulsant Effects of Novel Histamine $\mathrm{H}_{3} \mathrm{R}$ Antagonists in Electrically and Chemically Induced Seizures in Rats. Int J Mol Sci 19, 3386. https://doi.org/10.3390/ijms19113386.

Alloisio, S., Nobile, M. and Novellino, A. (2015). Multiparametric characterisation of neuronal network activity for in vitro agrochemical neurotoxicity assessment. Neurotoxicology $48,152-$ 165. https://doi.org/10.1016/j.neuro.2015.03.013.

Aminoshariae, A. and Khan, A. (2015). Acetaminophen: Old Drug, New Issues. J Endod 41, 588-593. https://doi.org/10.1016/j.joen.2015.01.024.

Amiri, M., Hosseinmardi, N., Bahrami, F. et al. (2013). Astrocyte- neuron interaction as a mechanism responsible for generation of neural synchrony: a study based on modeling and experiments. J Comput Neurosci 34, 489-504. https://doi.org/10.1007/s10827-012-0432-6.

Anson, B. D., Kolaja, K. L. and Kamp, T. J. (2011). Opportunities for use of human iPS cells in predictive toxicology. Clin Pharmacol Ther 89, 754-758. https://doi.org/10.1038/clpt.2011.9.

Antonio, L. L., Anderson, M. L., Angamo, E. A. et al. (2016). In vitro seizure like events and changes in ionic concentration. J Neurosci Methods 260, 33-44. https://doi.org/10.1016/j. jneumeth.2015.08.014.

Antunes Fernandes, E. C., Hendriks, H. S., van Kleef, R. G. D. M. et al. (2010). Activation and Potentiation of Human GABAA Receptors by Non-Dioxin-Like PCBs Depends on Chlorination Pattern. Toxicol Sci 118, 183-190. https://doi.org/10.1093/toxsci/kfq257.

Antunes Fernandes, E. C., Hendriks, H. S., van Kleef, R. G. D. M. et al. (2010). Potentiation of the Human GABA A Receptor As a Novel Mode of Action of Lower-Chlorinated Non-Dioxin-Like PCBs $\uparrow$. Environ Sci Technol 44, 2864-2869. https://doi.org/10.1021/es902321a.

Arrowsmith, J. and Miller, P. (2013). Phase II and Phase III attrition rates 2011-2012. Nat Rev Drug Discov 12, 569-569. https://doi.org/10.1038/nrd4090.

Authier, S., Arezzo, J., Delatte, M. S. et al. (2016). Safety pharmacology investigations on the nervous system: An industry survey. J Pharmacol Toxicol Methods 81, 37-46. https://doi.org/10.1016/J. VASCN.2016.06.001.

Avior, Y., Sagi, I. and Benvenisty, N. (2016). Pluripotent stem cells in disease modelling and drug discovery. Nat Rev Mol Cell Biol 17, 170-182. https://doi.org/10.1038/nrm.2015.27.

Bal-Price, A. K., Suñol, C., Weiss, D. G. et al. (2008). Application of in vitro neurotoxicity testing for regulatory purposes: Symposium III summary and research needs. Neurotoxicology 29, 520-531. https://doi.org/10.1016/j.neuro.2008.02.008.

Balestrino, R. and Schapira, A. H. V. (2019). Parkinson disease. Eur J Neurol, ene.14108. https://doi. org/10.1111/ene.14108.

Barclay, J. W., Morgan, A. and Burgoyne, R. D. (2005). Calcium-dependent regulation of exocytosis. Cell Calcium. https://doi.org/10.1016/j.ceca.2005.06.012.

Bardy, C., Van Den Hurk, M., Eames, T. et al. (2015). Neuronal medium that supports basic synaptic functions and activity of human neurons in vitro. Proc Natl Acad Sci U S A 112, E2725-E2734. https://doi.org/10.1073/pnas.1504393112. 
Barnard, E. A., Miledi, R. and Sumikawa, K. (1982). Translation of exogenous messenger RNA coding for nicotinic acetylcholine receptors produces functional receptors in Xenopus oocytes. Proc R Soc London - Biol Sci 215, 241-246. https://doi.org/10.1098/rspb.1982.0040.

Ben-Ari, Y. (2002). Excitatory actions of GABA during development: The nature of the nurture. Nat Rev Neurosci 3, 728-739. https://doi.org/10.1038/nrn920.

van Berlo-van de Laar, I. R. F., Arbouw, M. E. L. and Bles, C. M. A. (2015). Strychnine poisoning: uncommon, but does still happen. Ned Tijdschr Geneeskd 159, A8877. Available at: http://www. ncbi.nlm.nih.gov/pubmed/26173662 [Accessed September 17, 2019].

Berntsen, H. F., Bjørklund, C. G., Audinot, J.-N. et al. (2017). Time-dependent effects of perfluorinated compounds on viability in cerebellar granule neurons: Dependence on carbon chain length and functional group attached. Neurotoxicology 63, 70-83. https://doi. org/10.1016/j.neuro.2017.09.005.

Berntsen, H. F., Bjørklund, C. G., Strandabø, R. et al. (2018). PFOS-induced excitotoxicity is dependent on Ca2+ influx via NMDA receptors in rat cerebellar granule neurons. Toxicol Appl Pharmacol 357, 19-32. https://doi.org/10.1016/j.taap.2018.08.015.

Boyd-Kimball, D., Gonczy, K., Lewis, B. et al. (2018). Classics in Chemical Neuroscience: Chlorpromazine. ACS Chem Neurosci, acschemneuro.8b00258. https://doi.org/10.1021/ acschemneuro.8bo0258.

Bradley, J. A., Luithardt, H. H., Metea, M. R. et al. (2018). In Vitro Screening for Seizure Liability Using Microelectrode Array Technology. Toxicol Sci 163, 240-253. https://doi.org/10.1093/toxsci/ kfyo29.

Bradley, J. A. and Strock, C. J. (2019). Screening for Neurotoxicity with Microelectrode Array. Curr Protoc Toxicol 79, e67. https://doi.org/10.1002/cptx.67.

Brown, D. D. (2004). A Tribute to the Xenopus laevis Oocyte and Egg. https://doi.org/10.1074/jbc. $\mathrm{X} 400008200$.

Brown, J. P., Hall, D., Frank, C. L. et al. (2016). Evaluation of a microelectrode array-based assay for neural network ontogeny using training set chemicals. Toxicol Sci 154, 126-139. https://doi. org/10.1093/toxsci/kfw147.

Burn, D. J., Tomson, C. R. V., Seviour, J. et al. (1989). Strychnine poisoning as an unusual cause of convulsions. Postgrad Med J 65, 563-564. https://doi.org/10.1136/pgmj.65.766.563.

Buskila, Y., Breen, P. P., Tapson, J. et al. (2015). Extending the viability of acute brain slices. Sci Rep 4, 5309. https://doi.org/10.1038/srepo5309.

Butenhoff, J. L., Ehresman, D. J., Chang, S.-C. et al. (2009). Gestational and lactational exposure to potassium perfluorooctanesulfonate (K+PFOS) in rats: Developmental neurotoxicity. Reprod Toxicol 27, 319-330. https://doi.org/10.1016/j.reprotox.2008.12.010.

Carpenter, T. S., Lau, E. Y. and Lightstone, F. C. (2013). Computational Probing of a Modulatory Binding Site on the GABAA-Receptor Using Picrotoxin. Chem Res Toxicol. https://doi. org $/ 10.1021 / \mathrm{tx} 400167 \mathrm{~b}$.

Cellular Dynamics International iCell GlutaNeurons | Cellular Dynamics. Available at: https:// fujifilmcdi.com/products-services/icell-products/glutaneurons/ [Accessed January 3, 2020].

Chakraborty, S., Lennon, J. C., Malkaram, S. A. et al. (2019). Serotonergic system, cognition, and BPSD in Alzheimer's disease. Neurosci Lett 704, 36-44. https://doi.org/10.1016/j. neulet.2019.03.050.

Chen, N., Li, J., Li, D. et al. (2014). Chronic exposure to perfluorooctane sulfonate induces behavior defects and neurotoxicity through oxidative damages, in vivo and in vitro. J. Kim (ed.),. PLoS One 9, el13453. https://doi.org/10.1371/journal.pone.0113453. 
Clapham, D. E. (2007). Calcium Signaling. Cell 131, 1047-1058. https://doi.org/10.1016/J. CELL.2007.11.028.

Clarke, L. E. and Barres, B. A. (2013). Emerging roles of astrocytes in neural circuit development. Nat Rev Neurosci 14, 311-321. https://doi.org/10.1038/nrn3484.

Cleland, J. C. and Griggs, R. C. (2007). Channelopathies of the Nervous System. In Neurobiology of Disease (319-332). Elsevier. https://doi.org/10.1016/B978-012088592-3/50033-5.

Clowry, G., Molnár, Z. and Rakic, P. (2010). Renewed focus on the developing human neocortex. J Anat 217, 276-288. https://doi.org/10.1111/j.1469-7580.2010.01281.x.

Cotterill, E., Charlesworth, P., Thomas, C. W. et al. (2016). A comparison of computational methods for detecting bursts in neuronal spike trains and their application to human stem cell-derived neuronal networks. J Neurophysiol 116, 306-321. https://doi.org/10.1152/jn.00093.2016.

Cotterill, E., Hall, D., Wallace, K. et al. (2016). Characterization of Early Cortical Neural Network Development in Multiwell Microelectrode Array Plates. J Biomol Screen 21, 510-9. https://doi. org/10.1177/1087057116640520.

Croom, E. L., Shafer, T. J., Evans, M. V. et al. (2015). Improving in vitro to in vivo extrapolation by incorporating toxicokinetic measurements: A case study of lindane-induced neurotoxicity. Toxicol Appl Pharmacol 283, 9-19. https://doi.org/10.1016/j.taap.2014.11.006.

D'Aiuto, L., Zhi, Y., Kumar Das, D. et al. (2014). Large-scale generation of human iPSC-derived neural stem cells/early neural progenitor cells and their neuronal differentiation. Organogenesis 10, 365-77. https://doi.org/10.1080/15476278.2015.1011921.

D'Hulst, C., Atack, J. R. and Kooy, R. F. (2009). The complexity of the GABAA receptor shapes unique pharmacological profiles. Drug Discov Today 14, 866-875. https://doi.org/10.1016/J. DRUDIS.2009.06.009.

Dage, J. L., Colvin, E. M., Fouillet, A. et al. (2014). Pharmacological characterisation of ligandand voltage-gated ion channels expressed in human iPSC-derived forebrain neurons. Psychopharmacology (Berl) 231, 1105-1124. https://doi.org/10.1007/s00213-013-3384-2.

Defranchi, E., Novellino, A., Whelan, M. et al. (2011). Feasibility assessment of micro-electrode chip assay as a method of detecting neurotoxicity in vitro. Front Neuroeng, 1-12. https://doi. org/10.3389/fneng.2011.00006.

Dhir, A. (2012). Pentylenetetrazol (PTZ) Kindling Model of Epilepsy. In Current Protocols in Neuroscience (Unit9.37). Hoboken, NJ, USA: John Wiley \& Sons, Inc. https://doi. org/10.1002/0471142301.ns0937s58.

Dingemans, M. M. L., Schütte, M. G., Wiersma, D. M. M. et al. (2016). Chronic 14-day exposure to insecticides or methylmercury modulates neuronal activity in primary rat cortical cultures. Neurotoxicology 57, 194-202. https://doi.org/10.1016/j.neuro.2016.10.002.

Dolmetsch, R. and Geschwind, D. H. (2011). The human brain in a dish: The promise of iPSCderived neurons. Cell 145, 831-834. https://doi.org/10.1016/j.cell.2011.05.034.

Dugan, L. L., Bruno, V. M. G., Amagasu, S. M. et al. (1995). Glia modulate the response of murine cortical neurons to excitotoxicity: Glia exacerbate AMPA neurotoxicity. J Neurosci 15, 4545-4555. https://doi.org/10.1523/jneurosci.15-06-04545.1995.

Dutertre, S., Becker, C.-M. and Betz, H. (2012). Inhibitory glycine receptors: an update. J Biol Chem 287, 40216-23. https://doi.org/10.1074/jbc.R112.408229.

Easter, A., Bell, M. E., Damewood, J. R. et al. (2009). Approaches to seizure risk assessment in preclinical drug discovery. Drug Discov Today 14, 876-84. https://doi.org/10.1016/j. drudis.2009.06.003. 
Easter, A., Sharp, T. H., Valentin, J. P. et al. (2007). Pharmacological validation of a semi-automated in vitro hippocampal brain slice assay for assessment of seizure liability. J Pharmacol Toxicol Methods 56, 223-233. https://doi.org/10.1016/j.vascn.2007.04.008.

Ehresman, D. J., Froehlich, J. W., Olsen, G. W. et al. (2007). Comparison of human whole blood, plasma, and serum matrices for the determination of perfluorooctanesulfonate (PFOS), perfluorooctanoate (PFOA), and other fluorochemicals. Environ Res 103, 176-184. https://doi. org/10.1016/J.ENVRES.2006.06.008.

Emdad, L., D'Souza, S. L., Kothari, H. P. et al. (2012). Efficient differentiation of human embryonic and induced pluripotent stem cells into functional astrocytes. Stem Cells Dev. https://doi. org/10.1089/scd.2010.0560.

Engel, A. K., Fries, P. and Singer, W. (2001). Dynamic predictions: Oscillations and synchrony in top-down processing. Nat Rev Neurosci 2, 704-716. https://doi.org/10.1038/35094565.

Engle, S. J., Blaha, L. and Kleiman, R. J. (2018). Best Practices for Translational Disease Modeling Using Human iPSC-Derived Neurons. Neuron 100, 783-797. https://doi.org/10.1016/j. neuron.2018.10.033.

Environmental Protection Agency Federal Facilities Restoration, U. and Office, R. (2017). Technical Fact Sheet - Perfluorooctane Sulfonate (PFOS) and Perfluorooctanoic Acid (PFOA). Available at: https://www.epa.gov/sites/production/files/2017-12/documents/ffrrofactsheet_contaminants_ pfos_pfoa_11-20-17_508_0.pdf [Accessed July 29, 2019].

Evans, M. J. and Kaufman, M. H. (1981). Establishment in culture of pluripotential cells from mouse embryos. Nature 292, 154-156. https://doi.org/10.1038/292154aO.

Fan, J., Thalody, G., Kwagh, J. et al. (2019). Assessing seizure liability using multi-electrode arrays (MEA). Toxicol In Vitro 55, 93-100. https://doi.org/10.1016/j.tiv.2018.12.001.

Fellin, T. and Haydon, P. G. (2005). Do astrocytes contribute to excitation underlying seizures? Trends Mol Med 11, 530-533. https://doi.org/10.1016/j.molmed.2005.10.007.

Forns, J., Iszatt, N., White, R. A. et al. (2015). Perfluoroalkyl substances measured in breast milk and child neuropsychological development in a Norwegian birth cohort study. Environ Int 83, 176-182. https://doi.org/10.1016/J.ENVINT.2015.06.013.

Frank, C. L., Brown, J. P., Wallace, K. et al. (2017). From the Cover: Developmental Neurotoxicants Disrupt Activity in Cortical Networks on Microelectrode Arrays: Results of Screening 86 Compounds During Neural Network Formation. Toxicol Sci 160, 121-135. https://doi. org/10.1093/toxsci/kfx169.

Fromme, H., Tittlemier, S. A., Völkel, W. et al. (2009). Perfluorinated compounds - Exposure assessment for the general population in western countries. Int J Hyg Environ Health 212, 239270. https://doi.org/10.1016/J.IJHEH.2008.04.007.

Fuentes, S., Colomina, M. T., Vicens, P. et al. (2007). Concurrent Exposure to Perfluorooctane Sulfonate and Restraint Stress during Pregnancy in Mice: Effects on Postnatal Development and Behavior of the Offspring. Toxicol Sci 98, 589-598. https://doi.org/10.1093/toxsci/kfm121.

Fujii, S., Jia, Y., Yang, A. et al. (2000). Nicotine reverses GABAergic inhibition of long-term potentiation induction in the hippocampal CA1 region. Brain Res 863, 259-65. Available at: http://www.ncbi.nlm.nih.gov/pubmed/10773216 [Accessed January 7, 2019].

Fung, M., Thornton, A., Mybeck, K. et al. (2001). Evaluation of the Characteristics of Safety Withdrawal of Prescription Drugs from Worldwide Pharmaceutical Markets-1960 to 1999. Drug Inf J 35, 293-317. https://doi.org/10.1177/009286150103500134.

Gabriel, E. and Gopalakrishnan, J. (2017). Generation of ipsc-derived human brain organoids to model early neurodevelopmental disorders. J Vis Exp 2017. https://doi.org/10.3791/55372. 
Gao, M., Igata, H., Takeuchi, A. et al. (2017). Machine learning-based prediction of adverse drug effects: An example of seizure-inducing compounds. J Pharmacol Sci 133, 70-78. https://doi. org/10.1016/j.jphs.2017.01.003.

Gao, Z., Chen, Y., Cai, X. et al. (2016). Predict drug permeability to blood-brain-barrier from clinical phenotypes: drug side effects and drug indications. Bioinformatics 33, btw713. https:// doi.org/10.1093/bioinformatics/btw713.

Ghassabian, A., Bell, E. M., Ma, W. L. et al. (2018). Concentrations of perfluoroalkyl substances and bisphenol A in newborn dried blood spots and the association with child behavior. Environ Pollut 243, 1629-1636. https://doi.org/10.1016/j.envpol.2018.09.107.

Gochfeld, M. (2017). Sex Differences in Human and Animal Toxicology: Toxicokinetics. Toxicol Pathol 45, 172-189. https://doi.org/10.1177/0192623316677327.

Görtz, P., Fleischer, W., Rosenbaum, C. et al. (2004). Neuronal network properties of human teratocarcinoma cell line-derived neurons. Brain Res 1018, 18-25. https://doi.org/10.1016/j. brainres.2004.05.076.

Gotman, J. (2011). A few thoughts on "What is a seizure?" Epilepsy Behav 22, S2. https://doi. org/10.1016/j.yebeh.2011.08.025.

Goudarzi, H., Nakajima, S., Ikeno, T. et al. (2016). Prenatal exposure to perfluorinated chemicals and neurodevelopment in early infancy: The Hokkaido Study. Sci Total Environ 541, 1002-1010. https://doi.org/10.1016/j.scitotenv.2015.10.017.

Goulding, D. R., White, S. S., McBride, S. J. et al. (2017). Gestational exposure to perfluorooctanoic acid (PFOA): Alterations in motor related behaviors. Neurotoxicology 58, 110-119. https://doi. org/10.1016/j.neuro.2016.11.008.

Grainger, A. I., King, M. C., Nagel, D. A. et al. (2018). In vitro Models for Seizure-Liability Testing Using Induced Pluripotent Stem Cells. Front Neurosci 12, 590. https://doi.org/10.3389/ fnins.2018.00590.

Grandjean, P. and Clapp, R. (2015). Perfluorinated Alkyl Substances: Emerging Insights Into Health Risks. New Solut 25, 147-63. https://doi.org/10.1177/1048291115590506.

de Groot, M. W. G. D. M., Dingemans, M. M. L., Rus, K. H. et al. (2014). Characterization of calcium responses and electrical activity in differentiating mouse neural progenitor cells in vitro. Toxicol Sci 137, 428-35. https://doi.org/10.1093/toxsci/kft261.

De Groot, M. W. G. D. M., Van Kleef, R. G. D. M., De Groot, A. et al. (2016). In vitro developmental neurotoxicity following chronic exposure to $50 \mathrm{~Hz}$ extremely low-frequency electromagnetic fields in primary rat cortical cultures. Toxicol Sci 149, 433-440. https://doi.org/10.1093/toxsci/ kfv242.

de Groot, M. W. G. D. M., Westerink, R. H. S. and Dingemans, M. M. L. (2013). Don't judge a neuron only by its cover: Neuronal function in in vitro developmental neurotoxicity testing. Toxicol Sci 132, 1-7. https://doi.org/10.1093/toxsci/kfs269.

Gross, G. W. , Harsch, A., Rhoades, B. K. et al. (1997). Odor, drug and toxin analysis with neuronal networks in vitro: extracellular array recording of network responses. Biosens Bioelectron 12, 373-93. https://doi.org/10.1016/s0956-5663(97)00012-2.

Guan, B., Chen, X. and Zhang, H. (2013). Two-Electrode Voltage Clamp. Methods Mol Biol 998. https://doi.org/10.1007/978-1-62703-351-0_6.

Harada, K. H., Ishii, T. M., Takatsuka, K. et al. (2006). Effects of perfluorooctane sulfonate on action potentials and currents in cultured rat cerebellar Purkinje cells. Biochem Biophys Res Commun 351, 240-245. https://doi.org/10.1016/j.bbrc.2006.10.038. 
Harris, M. H., Oken, E., Rifas-Shiman, S. L. et al. (2018). Prenatal and childhood exposure to perand polyfluoroalkyl substances (PFASs) and child cognition. Environ Int 115, 358-369. https:// doi.org/10.1016/j.envint.2018.03.025.

Hartung, T. (2008). Thoughts on limitations of animal models. Park Relat Disord 14. https://doi. org/10.1016/j.parkreldis.2008.04.003.

Hay, M., Thomas, D. W., Craighead, J. L. et al. (2014). Clinical development success rates for investigational drugs. Nat Biotechnol 32, 40-51. https://doi.org/10.1038/nbt.2786.

Heikkilä, T. J., Ylä-Outinen, L., Tanskanen, J. M. A. et al. (2009). Human embryonic stem cellderived neuronal cells form spontaneously active neuronal networks in vitro. Exp Neurol 218, 109-116. https://doi.org/10.1016/j.expneurol.2009.04.011.

Hendriks, H. S., Antunes Fernandes, E. C., Bergman, Å. et al. (2010). PCB-47, PBDE-47, and 6-OHPBDE-47 Differentially Modulate Human GABAA and $\alpha 4 \beta_{2}$ Nicotinic Acetylcholine Receptors. Toxicol Sci 118, 635-642. https://doi.org/10.1093/toxsci/kfq284.

Hendriks, H. S., van Kleef, R. G. D. M., van den Berg, M. et al. (2012). Multiple Novel Modes of Action Involved in the In Vitro Neurotoxic Effects of Tetrabromobisphenol-A. Toxicol Sci 128, 235-246. https://doi.org/10.1093/toxsci/kfs136.

Heusinkveld, H. J., Thomas, G. O., Lamot, I. et al. (2010). Dual actions of lindane ( $\gamma$-hexachlorocyclohexane) on calcium homeostasis and exocytosis in rat PC12 cells. Toxicol Appl Pharmacol 248, 12-19. https://doi.org/10.1016/j.taap.2010.06.013.

Hodgkin, A. L., Huxley, A. F. and Katz, B. (1952). Measurement of current-voltage relations in the membrane of the giant axon of Loligo. J Physiol 116, 424-448. https://doi.org/10.1113/ jphysiol.1952.spoo4716.

Hogberg, H. T., Sobanski, T., Novellino, A. et al. (2011). Application of micro-electrode arrays (MEAs) as an emerging technology for developmental neurotoxicity: Evaluation of domoic acidinduced effects in primary cultures of rat cortical neurons. Neurotoxicology 32, 158-168. https:// doi.org/10.1016/j.neuro.2010.10.007.

Hondebrink, L., Hermans, E. J. P., Schmeink, S. et al. (2015). Structure-dependent inhibition of the human $\alpha_{1} \beta_{2} \gamma_{2}$ GABAA receptor by piperazine derivatives: A novel mode of action. Neurotoxicology 51, 1-9. https://doi.org/10.1016/j.neuro.2015.09.002.

Hondebrink, L., Kasteel, E. E. J., Tukker, A. M. et al. (2017). Neuropharmacological characterization of the new psychoactive substance methoxetamine. Neuropharmacology 123. https://doi. org/10.1016/j.neuropharm.2017.04.035.

Hondebrink, L., Verboven, A. H. A., Drega, W. S. et al. (2016). Neurotoxicity screening of (illicit) drugs using novel methods for analysis of microelectrode array (MEA) recordings. Neurotoxicology 55, 1-9. https://doi.org/10.1016/j.neuro.2016.04.020.

Hong, S. G., Dunbar, C. E. and Winkler, T. (2013). Assessing the risks of genotoxicity in the therapeutic development of induced pluripotent stem cells. Mol Ther 21, 272-281. https://doi. org/10.1038/mt.2012.255.

Høyer, B. B., Ramlau-Hansen, C. H., Obel, C. et al. (2015). Pregnancy serum concentrations of perfluorinated alkyl substances and offspring behaviour and motor development at age 5-9 years - a prospective study. Environ Heal 14, 2. https://doi.org/10.1186/1476-069X-14-2.

Hu, Y., Qu, Z.-Y., Cao, S.-Y. et al. (2016). Directed differentiation of basal forebrain cholinergic neurons from human pluripotent stem cells. J Neurosci Methods 266, 42-9. https://doi. org/10.1016/j.jneumeth.2016.03.017. 
Hyysalo, A., Ristola, M., Mäkinen, M. E. L. et al. (2017). Laminin $\alpha 5$ substrates promote survival, network formation and functional development of human pluripotent stem cell-derived neurons in vitro. Stem Cell Res 24, 118-127. https://doi.org/10.1016/j.scr.2017.09.002.

ICH (2001). ICH Topic S 7 A Safety Pharmacology Studies for Human Pharmaceuticals Step 5 NOTE FOR GUIDANCE ON SAFETY PHARMACOLOGY STUDIES FOR HUMAN PHARMACEUTICALS DATE FOR COMING INTO OPERATION. Available at: http://www.emea.eu.int [Accessed October 23, 2019].

Ishida, K., Tsuyama, Y., Sanoh, S. et al. (2017). Perfluorooctane sulfonate induces neuronal vulnerability by decreasing GluR2 expression. Arch Toxicol 91, 885-895. https://doi.org/10.1007/ s00204-016-1731-x.

Ishii, M. N., Yamamoto, K., Shoji, M. et al. (2017). Human induced pluripotent stem cell (hiPSC)derived neurons respond to convulsant drugs when co-cultured with hiPSC-derived astrocytes. Toxicology 389, 130-138. https://doi.org/10.1016/j.tox.2017.06.010.

Izhikevich, E. M., Desai, N. S., Walcott, E. C. et al. (2003). Bursts as a unit of neural information: selective communication via resonance. Trends Neurosci 26, 161-167. https://doi.org/10.1016/ So166-2236(03)00034-1.

Jenkins, M., Bilsland, J., Allsopp, T. E. et al. (2016). Patient-specific hiPSC bioprocessing for drug screening: Bioprocess economics and optimisation. Biochem Eng J 108, 84-97. https://doi. org/10.1016/j.bej.2015.09.024.

Jiruska, P., de Curtis, M., Jefferys, J. G. R. et al. (2013). Synchronization and desynchronization in epilepsy: controversies and hypotheses. J Physiol 591, 787-797. https://doi.org/10.1113/ jphysiol.2012.239590.

Johansson, N., Fredriksson, A. and Eriksson, P. (2008). Neonatal exposure to perfluorooctane sulfonate (PFOS) and perfluorooctanoic acid (PFOA) causes neurobehavioural defects in adult mice. Neurotoxicology 29, 160-9. https://doi.org/10.1016/j.neuro.2007.10.008.

Johnston, D. and Brown, T. H. (1984). The synaptic nature of the paroxysmal depolarizing shift in hippocampal neurons. Ann Neurol 16, S65-S71. https://doi.org/10.1002/ana.410160711.

Johnstone, A. F. M., Gross, G. W., Weiss, D. G. et al. (2010a). Microelectrode arrays: A physiologically based neurotoxicity testing platform for the 21st century. Neurotoxicology 31, 331-350. https:// doi.org/10.1016/j.neuro.2010.04.001.

Johnstone, A. F. M., Gross, G. W., Weiss, D. G. et al. (2010b). Microelectrode arrays: A physiologically based neurotoxicity testing platform for the 21st century $\times$. Neurotoxicology $31,331-350$. https:// doi.org/10.1016/j.neuro.2010.04.001.

Joshi, P., Bodnya, C., Ilieva, I. et al. (2019). Huntington's disease associated resistance to Mn neurotoxicity is neurodevelopmental stage and neuronal lineage dependent. Neurotoxicology 75, 148-157. https://doi.org/10.1016/j.neuro.2019.09.007.

Jun, S. B., Hynd, M. R., Dowell-Mesfin, N. et al. (2007). Low-density neuronal networks cultured using patterned poly-l-lysine on microelectrode arrays. J Neurosci Methods 160, 317-326. https://doi.org/10.1016/j.jneumeth.2006.09.009.

Juopperi, T. A., Kim, W. R., Chiang, C.-H. et al. (2012). Astrocytes generated from patient induced pluripotent stem cells recapitulate features of Huntington's disease patient cells. Mol Brain 5, 17. https://doi.org/10.1186/1756-6606-5-17.

Kapucu, F. E., Tanskanen, J. M. A., Mikkonen, J. E. et al. (2012). Burst analysis tool for developing neuronal networks exhibiting highly varying action potential dynamics. Front Comput Neurosci 6. https://doi.org/10.3389/fncom.2012.00038. 
Kapur, S., Cho, R., Jones, C. et al. (1999). Is amoxapine an atypical antipsychotic? positron-emission tomography investigation of its dopamine2 and serotoninz occupancy. Biol Psychiatry 45, 12171220. https://doi.org/10.1016/S0006-3223(98)00204-2.

Kasteel, E. E. J. and Westerink, R. H. S. (2017). Comparison of the acute inhibitory effects of Tetrodotoxin (TTX) in rat and human neuronal networks for risk assessment purposes. Toxicol Lett 270, 12-16. https://doi.org/10.1016/j.toxlet.2017.02.014.

Kawakami, J., Yamamoto, K., Asanuma, A. et al. (1997). Inhibitory Effect of New Quinolones on GABAAReceptor-Mediated Response and Its Potentiation with Felbinac inXenopusOocytes Injected with Mouse-Brain mRNA: Correlation with Convulsive Potencyin Vivo. Toxicol Appl Pharmacol 145, 246-254. https://doi.org/10.1006/TAAP.1997.8137.

Klioueva, I. A., Van Luijtelaar, E. L. J. M., Chepurnova, N. E. et al. (2001). PTZ-induced seizures in rats: Effects of age and strain. Physiol Behav 72, 421-426. https://doi.org/10.1016/S00319384(00)00425-X.

Konagaya, S., Ando, T., Yamauchi, T. et al. (2015). Long-term maintenance of human induced pluripotent stem cells by automated cell culture system. Sci Rep 5. https://doi.org/10.1038/ srep16647.

Kreir, M., De Bondt, A., Van den Wyngaert, I. et al. (2019). Role of Kv7.2/Kv7.3 and Mi muscarinic receptors in the regulation of neuronal excitability in hiPSC-derived neurons. Eur J Pharmacol, 172474. https://doi.org/10.1016/J.EJPHAR.2019.172474.

Kreir, M., Van Deuren, B., Versweyveld, S. et al. (2018). Do in vitro assays in rat primary neurons predict drug-induced seizure liability in humans? Toxicol Appl Pharmacol 346, 45-57. https:// doi.org/10.1016/J.TAAP.2018.03.028.

Krewski, D., Acosta, D., Andersen, M. et al. (2010). Toxicity Testing in the 21st Century: A Vision and a Strategy. J Toxicol Environ Heal Part B 13, 51-138. https://doi.org/10.1080/10937404.2010 .483176 .

Kuijlaars, J., Oyelami, T., Diels, A. et al. (2016). Sustained synchronized neuronal network activity in a human astrocyte co-culture system. Sci Rep 6. https://doi.org/10.1038/srep36529.

Kumlien, E. and Lundberg, P. O. (2010). Seizure risk associated with neuroactive drugs: Data from the WHO adverse drug reactions database. Seizure 19, 69-73. https://doi.org/10.1016/J. SEIZURE.2009.11.005.

Landrigan, P. J. (1999). Risk assessment for children and other sensitive populations. In Annals of the New York Academy of Sciences (1-9). New York Academy of Sciences. https://doi. org/10.1111/j.1749-6632.1999.tbo8073.x.

Laurenza, I., Pallocca, G., Mennecozzi, M. et al. (2013). A human pluripotent carcinoma stem cellbased model for in vitro developmental neurotoxicity testing: Effects of methylmercury, lead and aluminum evaluated by gene expression studies. Int J Dev Neurosci 31, 679-691. https://doi. org/10.1016/j.ijdevneu.2013.03.002.

Lee, H. -G., Lee, Y. J. and Yang, J.-H. (2012). Perfluorooctane sulfonate induces apoptosis of cerebellar granule cells via a ROS-dependent protein kinase C signaling pathway. Neurotoxicology 33, 314320. https://doi.org/10.1016/j.neuro.2012.01.017.

Legéndy, C. R. and Salcman, M. (1985). Bursts and recurrences of bursts in the spike trains of spontaneously active striate cortex neurons. J Neurophysiol 53, 926-939. https://doi.org/10.1152/ jn.1985.53.4.926.

Lein, P. J., Barnhart, C. D. and Pessah, I. N. (2011). Acute hippocampal slice preparation and hippocampal slice cultures. Methods Mol Biol 758, 115-34. https://doi.org/10.1007/978-1-61779170-3_8. 
Leite, P. E. C., Pereira, M. R., Harris, G. et al. (2019). Suitability of 3D human brain spheroid models to distinguish toxic effects of gold and poly-lactic acid nanoparticles to assess biocompatibility for brain drug delivery. Part Fibre Toxicol 16. https://doi.org/10.1186/s12989-019-0307-3.

Lenters, V., Iszatt, N., Forns, J. et al. (2019). Early-life exposure to persistent organic pollutants (OCPs, PBDEs, PCBs, PFASs) and attention-deficit/hyperactivity disorder: A multi-pollutant analysis of a Norwegian birth cohort. Environ Int 125, 33-42. https://doi.org/10.1016/j. envint.2019.01.020.

Lerche, H., Jurkat-Rott, K. and Lehmann-Horn, F. (2001). Ion channels and epilepsy. Am J Med Genet - Semin Med Genet 106, 146-159. https://doi.org/10.1002/ajmg.1582.

Li, X. J., Du, Z. W., Zarnowska, E. D. et al. (2005). Specification of motoneurons from human embryonic stem cells. Nat Biotechnol 23, 215-221. https://doi.org/10.1038/nbt1063.

Li, Y., Fletcher, T., Mucs, D. et al. (2018). Half-lives of PFOS, PFHxS and PFOA after end of exposure to contaminated drinking water. Occup Environ Med 75, 46-51. https://doi.org/10.1136/ oemed-2017-104651.

Li, Z., Liu, Q., Liu, C. et al. (2017). Evaluation of PFOS-mediated neurotoxicity in rat primary neurons and astrocytes cultured separately or in co-culture. Toxicol Vitr 38, 77-90. https://doi. org/10.1016/j.tiv.2016.11.002.

Liao, C.-Y., Cui, L., Zhou, Q.-F. et al. (2009). Effects of perfluorooctane sulfonate on ion channels and glutamate-activated current in cultured rat hippocampal neurons. Environ Toxicol Pharmacol 27, 338-44. https://doi.org/10.1016/j.etap.2008.11.013.

Liao, C.-Y., Li, X.-Y., Wu, B. et al. (2008). Acute enhancement of synaptic transmission and chronic inhibition of synaptogenesis induced by perfluorooctane sulfonate through mediation of voltage-dependent calcium channel. Environ Sci Technol 42, 5335-41. Available at: http://www. ncbi.nlm.nih.gov/pubmed/18754390 [Accessed January 7, 2019].

Liao, C., Wang, T., Cui, L. et al. (2009). Changes in synaptic transmission, calcium current, and neurite growth by perfluorinated compounds are dependent on the chain length and functional group. Environ Sci Technol 43, 2099-104. Available at: http://www.ncbi.nlm.nih.gov/ pubmed/19368220 [Accessed January 7, 2019].

Liew, Z., Ritz, B., Bach, C. C. et al. (2018). Prenatal Exposure to Perfluoroalkyl Substances and IQ Scores at Age 5; a Study in the Danish National Birth Cohort. Environ Health Perspect 126, 067004. https://doi.org/10.1289/EHP2754.

Liew, Z., Ritz, B., Bonefeld-Jørgensen, E. C. et al. (2014). Prenatal Exposure to Perfluoroalkyl Substances and the Risk of Congenital Cerebral Palsy in Children. Am J Epidemiol 180, 574-581. https://doi.org/10.1093/aje/kwu179.

Liew, Z., Ritz, B., von Ehrenstein, O. S. et al. (2015). Attention deficit/hyperactivity disorder and childhood autism in association with prenatal exposure to perfluoroalkyl substances: a nested case-control study in the Danish National Birth Cohort. Environ Health Perspect 123, 367-73. https://doi.org/10.1289/ehp.1408412.

Lindstrom, A. B., Strynar, M. J. and Libelo, E. L. (2011). Polyfluorinated Compounds: Past, Present, and Future. Environ Sci Technol 45, 7954-7961. https://doi.org/10.1021/es2011622.

Linville, R. M., DeStefano, J. G., Sklar, M. B. et al. (2019). Human iPSC-derived blood-brain barrier microvessels: validation of barrier function and endothelial cell behavior. Biomaterials 190-191, 24-37. https://doi.org/10.1016/j.biomaterials.2018.10.023.

Litovitz, T. L. and Troutman, W. G. (1983). Amoxapine overdose. Seizures and fatalities. JAMA 250, 1069-71. Available at: http://www.ncbi.nlm.nih.gov/pubmed/6876345 [Accessed November 14, 2019]. 
Little, D., Ketteler, R., Gissen, P. et al. (2019). Using stem cell-derived neurons in drug screening for neurological diseases. Neurobiol Aging 78, 130-141. https://doi.org/10.1016/J. NEUROBIOLAGING.2019.02.008.

Liu, H. and Zhang, S. C. (2011). Specification of neuronal and glial subtypes from human pluripotent stem cells. Cell Mol Life Sci 68, 3995-4008. https://doi.org/10.1007/s00018-011-0770-y.

Liu, X., Jin, Y., Liu, W. et al. (2011). Possible mechanism of perfluorooctane sulfonate and perfluorooctanoate on the release of calcium ion from calcium stores in primary cultures of rat hippocampal neurons. Toxicol Vitr 25, 1294-1301. https://doi.org/10.1016/j.tiv.2011.04.016.

Llorens, J., Li, A. A., Ceccatelli, S. et al. (2012). Strategies and tools for preventing neurotoxicity: To test, to predict and how to do it. Neurotoxicology 33, 796-804. https://doi.org/10.1016/j. neuro.2012.01.019.

Mack, C. M., Lin, B. J., Turner, J. D. et al. (2014). Burst and principal components analyses of MEA data for 16 chemicals describe at least three effects classes. Neurotoxicology 40, 75-85. https:// doi.org/10.1016/j.neuro.2013.11.008.

Mackenzie, L., Medvedev, A., Hiscock, J. J. et al. (2002). Picrotoxin-induced generalised convulsive seizure in rat: changes in regional distribution and frequency of the power of electroencephalogram rhythms. Clin Neurophysiol 113, 586-596. https://doi.org/10.1016/S13882457(02)00040-8.

Malik, N., Efthymiou, A. G., Mather, K. et al. (2014). Compounds with species and cell type specific toxicity identified in a 2000 compound drug screen of neural stem cells and rat mixed cortical neurons. Neurotoxicology 45, 192-200. https://doi.org/10.1016/j.neuro.2014.10.007.

Marchi, N., Oby, E., Batra, A. et al. (2007). In Vivo and In Vitro Effects of Pilocarpine: Relevance to Ictogenesis. Epilepsia 48, 1934-1946. https://doi.org/10.1111/j.1528-1167.2007.01185.x.

Mariussen, E. (2012). Neurotoxic effects of perfluoroalkylated compounds: mechanisms of action and environmental relevance. Arch Toxicol 86, 1349-1367. https://doi.org/10.1007/s00204-0120822-6.

Markram, H., Toledo-Rodriguez, M., Wang, Y. et al. (2004). Interneurons of the neocortical inhibitory system. Nat Rev Neurosci 5, 793-807. https://doi.org/10.1038/nrn1519.

Maroof, A. M., Keros, S., Tyson, J. A. et al. (2013). Directed differentiation and functional maturation of cortical interneurons from human embryonic stem cells. Cell Stem Cell 12, 559-572. https:// doi.org/10.1016/j.stem.2013.04.008.

Martín-López, D., Jiménez-Jiménez, D., Cabañés-Martínez, L. et al. (2017). The Role of Thalamus Versus Cortex in Epilepsy: Evidence from Human Ictal Centromedian Recordings in Patients Assessed for Deep Brain Stimulation. Int J Neural Syst 27, 1750010. https://doi.org/10.1142/ So129065717500101.

Martin, G. R. (1981). Isolation of a pluripotent cell line from early mouse embryos cultured in medium conditioned by teratocarcinoma stem cells. Proc Natl Acad Sci U S A 78, 7634-7638. https://doi.org/10.1073/pnas.78.12.7634.

Mashayekhi, V., Tehrani, K. H. M. E., Hashemzaei, M. et al. (2015). Mechanistic approach for the toxic effects of perfluorooctanoic acid on isolated rat liver and brain mitochondria. Hum Exp Toxicol 34, 985-96. https://doi.org/10.1177/0960327114565492.

Maslarova, A., Salar, S., Lapilover, E. et al. (2013). Increased susceptibility to acetylcholine in the entorhinal cortex of pilocarpine-treated rats involves alterations in KCNQ channels. Neurobiol Dis 56, 14-24. https://doi.org/10.1016/J.NBD.2013.02.016. 
Matsuda, N., Odawara, A., Katoh, H. et al. (2018). Detection of synchronized burst firing in cultured human induced pluripotent stem cell-derived neurons using a 4-step method. Biochem Biophys Res Commun 497, 612-618. https://doi.org/10.1016/j.bbrc.2018.02.117.

McConnell, E. R., McClain, M. A., Ross, J. et al. (2012). Evaluation of multi-well microelectrode arrays for neurotoxicity screening using a chemical training set. Neurotoxicology 33, 1048-1057. https://doi.org/10.1016/j.neuro.2012.05.001.

Meldrum, B. S. (2000). Glutamate as a Neurotransmitter in the Brain: Review of Physiology and Pathology. J Nutr 130, 1007S-1015S. https://doi.org/10.1093/jn/130.4.1007s.

Meneghello, G., Verheyen, A., Van Ingen, M. et al. (2015). Evaluation of established human iPSCderived neurons to model neurodegenerative diseases. Neuroscience 301, 204-212. https://doi. org/10.1016/j.neuroscience.2015.05.071.

Meyerson, J. R., Kumar, J., Chittori, S. et al. (2014). Structural mechanism of glutamate receptor activation and desensitization. Nature 514, 328-334. https://doi.org/10.1038/nature13603.

Nagao, T., Alonso, A. and Avoli, M. (1996). Epileptiform activity induced by pilocarpine in the rat hippocampal-entorhinal slice preparation. Neuroscience 72, 399-408. https://doi. org/10.1016/0306-4522(95)00534-x.

Neher, E. and Sakaba, T. (2008). Multiple Roles of Calcium Ions in the Regulation of Neurotransmitter Release. Neuron 59, 861-872. https://doi.org/10.1016/j.neuron.2008.08.019.

Neucyte Inc. (2019). NeuCyte | Data. Available at: https://www.neucyte.com/data [Accessed September 11, 2019].

Neve, K. A., Seamans, J. K. and Trantham-Davidson, H. (2004). Dopamine receptor signaling. In Journal of Receptors and Signal Transduction (165-205). https://doi.org/10.1081/RRS200029981.

Nicolas, J., Hendriksen, P. J. M., van Kleef, R. G. D. M. et al. (2014). Detection of marine neurotoxins in food safety testing using a multielectrode array. Mol Nutr Food Res 58, 2369-2378. https://doi. org/10.1002/mnfr.201400479.

Nicolson, T. J., Mellor, H. R. and Roberts, R. R. A. (2010). Gender differences in drug toxicity. Trends Pharmacol Sci 31, 108-114. https://doi.org/10.1016/j.tips.2009.12.001.

Nishiyama, Y., Iwanami, A., Kohyama, J. et al. (2016). Safe and efficient method for cryopreservation of human induced pluripotent stem cell-derived neural stem and progenitor cells by a programmed freezer with a magnetic field. Neurosci Res 107, 20-29. https://doi.org/10.1016/j. neures.2015.11.011.

Nistor, G. I., Totoiu, M. O., Haque, N. et al. (2005). Human embryonic stem cells differentiate into oligodendrocytes in high purity and myelinate after spinal cord transplantation. Glia. https:// doi.org/10.1002/glia.20127.

Niswender, C. M. and Conn, P. J. (2010). Metabotropic Glutamate Receptors: Physiology, Pharmacology, and Disease. Annu Rev Pharmacol Toxicol 50, 295-322. https://doi.org/10.1146/ annurev.pharmtox.011008.145533.

Nolan, L. and O'Malley, K. (1988). Prescribing for the Elderly Part I: Sensitivity of the Elderly to Adverse Drug Reactions*. J Am Geriatr Soc 36, 142-149. https://doi.org/10.1111/j.1532-5415.1988. tbo1785.x.

Novellino, A., Scelfo, B., Palosaari, T. et al. (2011). Development of Micro-Electrode Array Based Tests for Neurotoxicity: Assessment of Interlaboratory Reproducibility with Neuroactive Chemicals. Front Neuroeng 4, 4. https://doi.org/10.3389/fneng.2011.00004. 
Ochalek, A., Mihalik, B., Avci, H. X. et al. (2017). Neurons derived from sporadic Alzheimer's disease iPSCs reveal elevated TAU hyperphosphorylation, increased amyloid levels, and GSK3B activation. Alzheimer's Res Ther 9. https://doi.org/10.1186/s13195-017-0317-z.

Odawara, A., Katoh, H., Matsuda, N. et al. (2016). Physiological maturation and drug responses of human induced pluripotent stem cell-derived cortical neuronal networks in long-term culture. Sci Rep 6. https://doi.org/10.1038/srep26181.

Odawara, A., Matsuda, N., Ishibashi, Y. et al. (2018). Toxicological evaluation of convulsant and anticonvulsant drugs in human induced pluripotent stem cell-derived cortical neuronal networks using an MEA system. Sci Rep 8, 10416. https://doi.org/10.1038/s41598-018-28835-7.

Odawara, A., Saitoh, Y., Alhebshi, A. H. et al. (2014). Long-term electrophysiological activity and pharmacological response of a human induced pluripotent stem cell-derived neuron and astrocyte co-culture. Biochem Biophys Res Commun 443, 1176-1181. https://doi.org/10.1016/j. bbrc.2013.12.142.

Olsen, G. W., Burris, J. M., Ehresman, D. J. et al. (2007). Half-life of serum elimination of perfluorooctanesulfonate,perfluorohexanesulfonate, and perfluorooctanoate in retired fluorochemical production workers. Environ Health Perspect 115, 1298-305. https://doi. org/10.1289/ehp.10009.

Olsen, R. W. and Sieghart, W. (2009). GABAA receptors: Subtypes provide diversity of function and pharmacology. Neuropharmacology 56, 141-148. https://doi.org/10.1016/j. neuropharm.2008.07.045.

Olsen, R. W. and Sieghart, W. (2008). International Union of Pharmacology. LXX. Subtypes of $\gamma$-aminobutyric acidA receptors: Classification on the basis of subunit composition, pharmacology, and function. Update. Pharmacol Rev 60, 243-260. https://doi.org/10.1124/ pr.108.00505.

Olson, H., Betton, G., Robinson, D. et al. (2000). Concordance of the Toxicity of Pharmaceuticals in Humans and in Animals. Regul Toxicol Pharmacol 32, 56-67. https://doi.org/10.1006/ rtph.2000.1399.

Onakpoya, I. J., Heneghan, C. J. and Aronson, J. K. (2016). Post-marketing withdrawal of 462 medicinal products because of adverse drug reactions: a systematic review of the world literature. BMC Med 14, 10. https://doi.org/10.1186/s12916-016-0553-2.

Onishchenko, N., Fischer, C., Wan Ibrahim, W. N. et al. (2011). Prenatal exposure to PFOS or PFOA alters motor function in mice in a sex-related manner. Neurotox Res 19, 452-61. https://doi. org/10.1007/s12640-010-9200-4.

Owen-Smith, J., Scott, C. T. and McCormick, J. B. (2012). Expand and regularize federal funding for human pluripotent stem cell research. J Policy Anal Manage 31, 714-22. Available at: http://www. ncbi.nlm.nih.gov/pubmed/22764380 [Accessed October 25, 2019].

Paavilainen, T., Pelkonen, A., Mäkinen, M. E.-L. et al. (2018). Effect of prolonged differentiation on functional maturation of human pluripotent stem cell-derived neuronal cultures. Stem Cell Res 27. https://doi.org/10.1016/j.scr.2018.01.018.

Pamies, D., Block, K., Lau, P. et al. (2018). Rotenone exerts developmental neurotoxicity in a human brain spheroid model. Toxicol Appl Pharmacol 354, 101-114. https://doi.org/10.1016/j. taap.2018.02.003.

Pamies, D. and Hartung, T. (2017). 21st Century Cell Culture for 21st Century Toxicology. Chem Res Toxicol 30, 43-52. https://doi.org/10.1021/acs.chemrestox.6boo269. 
Peña, F. and Tapia, R. (2000a). Seizures and neurodegeneration induced by 4 -aminopyridine in rat hippocampus in vivo: role of glutamate- and GABA-mediated neurotransmission and of ion channels. Neuroscience 101, 547-561. https://doi.org/10.1016/S0306-4522(00)00400-0.

Peña, F. and Tapia, R. (2000b). Seizures and neurodegeneration induced by 4 -aminopyridine in rat hippocampus in vivo: role of glutamate- and GABA-mediated neurotransmission and of ion channels. Neuroscience 101, 547-561. https://doi.org/10.1016/S0306-4522(00)00400-0.

Perrier, A. L., Tabar, V., Barberi, T. et al. (2004). Derivation of midbrain dopamine neurons from human embryonic stem cells. Available at: www.pnas.orgcgidoi1o.1073pnas.0404700101 [Accessed October 25, 2019].

Petroff, O.A. C. (2002). GABA and glutamate in the human brain. Neuroscientist 8, 562-73. https:// doi.org/10.1177/1073858402238515.

Picciotto, M. R., Higley, M. J. and Mineur, Y. S. (2012). Acetylcholine as a neuromodulator: cholinergic signaling shapes nervous system function and behavior. Neuron 76, 116-29. https:// doi.org/10.1016/j.neuron.2012.08.036.

Pickett, T. A. and Enns, R. (1996). Atypical Presentation of 4-Aminopyridine Overdose. Ann Emerg Med 27, 382-385. https://doi.org/10.1016/So196-0644(96)70277-9.

Pin, J.-P. and Prezeau, L. (2007). Allosteric Modulators of GABAB Receptors: Mechanism of Action and Therapeutic Perspective. Curr Neuropharmacol 5, 195-201. https://doi. org/10.2174/157015907781695919.

Pinkas, A., Slotkin, T. A., Brick-Turin, Y. et al. (2010). Neurobehavioral teratogenicity of perfluorinated alkyls in an avian model. Neurotoxicol Teratol 32, 182-186. https://doi. org/10.1016/j.ntt.2009.11.004.

Pleil, J. D. (2016). Comparing biomarker measurements to a normal range: When to use standard error of the mean (SEM) or standard deviation (SD) confidence intervals tests. Biomarkers 21, 195-199. https://doi.org/10.3109/1354750X.2015.1134666.

Preininger, M. K., Singh, M. and Xu, C. (2016). Cryopreservation of human pluripotent stem cell-derived cardiomyocytes: Strategies, challenges, and future directions. In Advances in Experimental Medicine and Biology (123-135). Springer New York LLC. https://doi. org/10.1007/978-3-319-45457-3_10.

Prince, M., Wimo, A., Guerchet, M. et al. (2015). World Alzheimer Report 2015: The Global Impact of Dementia - An analysis of prevalence, incidence, cost and trends. Alzheimer's Dis Int, 84. https://doi.org/10.1111/j.0963-7214.2004.00293.x.

Qi, Y., Zhang, X. J., Renier, N. et al. (2017). Combined small-molecule inhibition accelerates the derivation of functional cortical neurons from human pluripotent stem cells. Nat Biotechnol 35 , 154-163. https://doi.org/10.1038/nbt.3777.

Qiu, C., Johnson, B. N. and Tallent, M. K. (2007). K+ M-Current Regulates the Transition to Seizures in Immature and Adult Hippocampus. Epilepsia 48, 2047-2058. https://doi.org/10.1111/j.15281167.2007.01193.x.

Quaak, I., de Cock, M., de Boer, M. et al. (2016). Prenatal exposure to perfluoroalkyl substances and Behavioral development in children. Int J Environ Res Public Health 13. https://doi.org/10.3390/ ijerph13050511.

Raposo, J., Teotónio, R., Bento, C. et al. (2016). Amoxicillin, a potential epileptogenic drug. Epileptic Disord 18, 454-457. https://doi.org/10.1684/epd.2016.0875.

Reistad, T., Fonnum, F. and Mariussen, E. (2013). Perfluoroalkylated compounds induce cell death and formation of reactive oxygen species in cultured cerebellar granule cells. Toxicol Lett 218, 56-60. https://doi.org/10.1016/j.toxlet.2013.01.006. 
Represa, A. and Ben-Ari, Y. (2005). Trophic actions of GABA on neuronal development. Trends Neurosci 28, 278-283. https://doi.org/10.1016/j.tins.2005.03.010.

Reubinoff, B. E., Itsykson, P., Turetsky, T. et al. (2001). Neural progenitors from human embryonic stem cells. Nat Biotechnol 19, 1134-1140. https://doi.org/10.1038/nbt1201-1134.

Richards, G. R., Smith, A. J., Parry, F. et al. (2006). A morphology- and kinetics-based cascade for human neural cell high content screening. Assay Drug Dev Technol 4, 143-52. https://doi. org/10.1089/adt.2006.4.143.

Robertson, J. A. (2010). Embryo stem cell research: Ten years of controversy. In Journal of Law, Medicine and Ethics (191-203). https://doi.org/10.1111/j.1748-720X.2010.00479.x.

Robinette, B. L., Harrill, J. a, Mundy, W. R. et al. (2011). In Vitro Assessment of Developmental Neurotoxicity: Use of Microelectrode Arrays to Measure Functional Changes in Neuronal Network Ontogeny1. Front Neuroeng 4, 1. https://doi.org/10.3389/fneng.2011.00001.

Robinton, D. A. and Daley, G. Q. (2012). The promise of induced pluripotent stem cells in research and therapy. Nature 481, 295-305. https://doi.org/10.1038/nature10761.

Rogawski, M. A. and Löscher, W. (2004). The neurobiology of antiepileptic drugs. Nat Rev Neurosci 5, 553-564. https://doi.org/10.1038/nrn1430.

Romanelli, R. J., Williams, J. T. and Neve, K. A. (2010). Dopamine Receptor Signaling: Intracellular Pathways to Behavior. In The Dopamine Receptors (137-173). Humana Press. https://doi. org/10.1007/978-1-60327-333-6_6.

Roses, A. D. (2008). Pharmacogenetics in drug discovery and development: A translational perspective. Nat Rev Drug Discov 7, 807-817. https://doi.org/10.1038/nrd2593.

Routledge, P. A., O'Mahony, M. S. and Woodhouse, K. W. (2003). Adverse drug reactions in elderly patients. Br J Clin Pharmacol 57, 121-126. https://doi.org/10.1046/j.1365-2125.2003.01875.x.

Sakmann, B. and Neher, E. (1984). Patch Clamp Techniques for Studying Ionic Channels in Excitable Membranes. Annu Rev Physiol 46, 455-472. https://doi.org/10.1146/annurev.physiol.46.1.455.

De Sarro, A., Zappalá, M., Chimirri, A. et al. (1993). Quinolones potentiate cefazolin-induced seizures in $\mathrm{DBA} / 2$ mice. Antimicrob Agents Chemother 37, 1497-503. https://doi.org/10.1128/ AAC.37.7.1497.

Sasaki, T., Suzuki, I., Yokoi, R. et al. (2019a). Synchronous spike patterns in differently mixed cultures of human iPSC-derived glutamatergic and GABAergic neurons. Biochem Biophys Res Commun. https://doi.org/10.1016/J.BBRC.2019.03.161.

Sasaki, T., Suzuki, I., Yokoi, R. et al. (2019b). Synchronous spike patterns in differently mixed cultures of human iPSC-derived glutamatergic and GABAergic neurons. Biochem Biophys Res Commun 513, 300-305. https://doi.org/10.1016/j.bbrc.2019.03.161.

Scharfman, H. E. (2007). The neurobiology of epilepsy. Curr Neurol Neurosci Rep 7, 348-354. https://doi.org/10.1007/s11910-007-0053-z.

Schöndorf, D. C., Aureli, M., McAllister, F. E. et al. (2014). IPSC-derived neurons from GBA1associated Parkinson's disease patients show autophagic defects and impaired calcium homeostasis. Nat Commun 5. https://doi.org/10.1038/ncomms5028.

Schwartzentruber, J., Foskolou, S., Kilpinen, H. et al. (2017). Molecular and functional variation in iPSC-derived sensory neurons. Nat Genet. https://doi.org/10.1038/s41588-017-0005-8.

Shafer, T. J., Rijal, S. O. and Gross, G. W. (2008). Complete inhibition of spontaneous activity in neuronal networks in vitro by deltamethrin and permethrin. Neurotoxicology 29, 203-212. https://doi.org/10.1016/j.neuro.2008.01.002. 
Sharma, R. P., Schuhmacher, M. and Kumar, V. (2017). Developing integrated PBPK/PD coupled mechanistic pathway model (miRNA-BDNF): An approach towards system toxicology. Toxicol Lett 280, 79-91. https://doi.org/10.1016/j.toxlet.2017.08.003.

Sherman, S. P. and Bang, A. G. (2018). High-throughput screen for compounds that modulate neurite growth of human induced pluripotent stem cell-derived neurons. DMM Dis Model Mech 11, 5DUMMY. https://doi.org/10.1242/dmm.031906.

Sigel, E. and Minier, F. (2005). The Xenopus oocyte: System for the study of functional expression and modulation of proteins. Mol Nutr Food Res 49, 228-234. https://doi.org/10.1002/ mnfr.200400104.

Sigel, E. and Steinmann, M. E. (2012). Structure, function, and modulation of GABAA receptors. J Biol Chem 287, 40224-40231. https://doi.org/10.1074/jbc.R112.386664.

Simpson, K. J. and Brodie, M. J. (1985). Convulsions related to enoxacin. Lancet 326, 161. https://doi. org/10.1016/S0140-6736(85)90270-3.

Singh, N., Saha, L., Kumari, P. et al. (2019). Effect of dimethyl fumarate on neuroinflammation and apoptosis in pentylenetetrazol kindling model in rats. Brain Res Bull 144, 233-245. https://doi. org/10.1016/j.brainresbull.2018.11.013.

Slotkin, T. A., MacKillop, E. A., Melnick, R. L. et al. (2008). Developmental Neurotoxicity of Perfluorinated Chemicals Modeled in Vitro. Environ Health Perspect 116, 716-722. https://doi. org/10.1289/ehp.11253.

Smetters, D., Majewska, A. and Yuste, R. (1999). Detecting action potentials in neuronal populations with calcium imaging. Methods A Companion to Methods Enzymol 18, 215-221. https://doi. org/10.1006/meth.1999.0774.

Spulber, S., Kilian, P., Wan Ibrahim, W. N. et al. (2014). PFOS Induces Behavioral Alterations, Including Spontaneous Hyperactivity That Is Corrected by Dexamfetamine in Zebrafish Larvae F. Del Bene (ed.),. PLoS One 9, e94227. https://doi.org/10.1371/journal.pone.0094227.

Steffenhagen, C., Kraus, S., Dechant, F.-X. et al. (2011). Identity, fate and potential of cells grown as neurospheres: species matters. Stem cell Rev reports 7, 815-35. https://doi.org/10.1007/s12015011-9251-9.

Strickland, J. D., LeFew, W. R., Crooks, J. et al. (2016). In vitro screening of metal oxide nanoparticles for effects on neural function using cortical networks on microelectrode arrays. Nanotoxicology 10, 619-628. https://doi.org/10.3109/17435390.2015.1107142.

Strøm, M., Hansen, S., Olsen, S. F. et al. (2014). Persistent organic pollutants measured in maternal serum and offspring neurodevelopmental outcomes - A prospective study with long-term follow-up. Environ Int 68, 41-48. https://doi.org/10.1016/j.envint.2014.03.002.

Südhof, T. C. (2014). The Molecular Machinery of Neurotransmitter Release (Nobel Lecture). Angew Chemie Int Ed 53, 12696-12717. https://doi.org/10.1002/anie.201406359.

Sun, A. X., Ng, H.-H. and Tan, E.-K. (2018). Translational potential of human brain organoids. Ann Clin Transl Neurol 5, 226-235. https://doi.org/10.1002/acn3.505.

Sun, P., Nie, X., Chen, X. et al. (2018). Nrf2 Signaling Elicits a Neuroprotective Role Against PFOS-mediated Oxidative Damage and Apoptosis. Neurochem Res 43, 2446-2459. https://doi. org/10.1007/s11064-018-2672-y.

Swistowski, A., Peng, J., Liu, Q. et al. (2010). Efficient generation of functional dopaminergic neurons from human induced pluripotent stem cells under defined conditions. Stem Cells 28 , 1893-1904. https://doi.org/10.1002/stem.499. 
Takahashi, K. and Yamanaka, S. (2006). Induction of Pluripotent Stem Cells from Mouse Embryonic and Adult Fibroblast Cultures by Defined Factors. Cell 126, 663-676. https://doi.org/10.1016/j. cell.2006.07.024.

Takemoto, T., Ishihara, Y., Ishida, A. et al. (2015). Neuroprotection elicited by nerve growth factor and brain-derived neurotrophic factor released from astrocytes in response to methylmercury. Environ Toxicol Pharmacol 40, 199-205. https://doi.org/10.1016/j.etap.2015.06.010.

Tang, X., Zhou, L., Wagner, A. M. et al. (2013). Astroglial cells regulate the developmental timeline of human neurons differentiated from induced pluripotent stem cells. Stem Cell Res 11, 743-757. https://doi.org/10.1016/j.scr.2013.05.002.

Thomson, J. A., Itskovitz-Eldro, J., Shapiro, S. S. et al. (1998). Embryonic stem cell lines derived from human blastocysts. Science (80-) 282, 1145-1147. https://doi.org/10.1126/science.282.5391.1145.

Van Thriel, C., Westerink, R. H. S., Beste, C. et al. (2012). Translating neurobehavioural endpoints of developmental neurotoxicity tests into in vitro assays and readouts. Neurotoxicology 33 , 911924. https://doi.org/10.1016/j.neuro.2011.10.002.

Traynelis, S. F., Wollmuth, L. P., McBain, C. J. et al. (2010). Glutamate receptor ion channels: Structure, regulation, and function. Pharmacol Rev 62, 405-496. https://doi.org/10.1124/ pr.109.002451.

Tukker, A. M., De Groot, M. W. G. D. M., Wijnolts, F. M. J. et al. (2016). Is the time right for in vitro neurotoxicity testing using human iPSC-derived neurons? ALTEX 33, 261-271. https://doi. org/10.14573/altex.1510091.

Tukker, A. M., Van Kleef, R. G. D. M., Wijnolts, F. M. J. et al. (2020). Towards animal-free neurotoxicity screening: Applicability of hiPSC-derived neuronal models for in vitro seizure liability assessment. ALTEX 37, 121-135. https://doi.org/10.14573/altex.1907121.

Tukker, A. M., Wijnolts, F. M. J., de Groot, A. et al. (2018). Human iPSC-derived neuronal models for in vitro neurotoxicity assessment. Neurotoxicology 67, 215-225. https://doi.org/10.1016/J. NEURO.2018.06.007.

Tukker, A. M., Wijnolts, F. M. J., de Groot, A. et al. (2019). In Vitro Techniques for Assessing Neurotoxicity Using Human iPSC-Derived Neuronal Models. In (17-35). Humana, New York, NY. https://doi.org/10.1007/978-1-4939-9228-7_2.

Ulhaq, M., Orn, S., Carlsson, G. et al. (2013). Locomotor behavior in zebrafish (Danio rerio) larvae exposed to perfluoroalkyl acids. Aquat Toxicol 144-145, 332-40. https://doi.org/10.1016/j. aquatox.2013.10.021.

Vadodaria, K. C., Stern, S., Marchetto, M. C. et al. (2018). Serotonin in psychiatry: in vitro disease modeling using patient-derived neurons. Cell Tissue Res 371, 161-170. https://doi.org/10.1007/ s00441-017-2670-4.

Valdivia, P., Martin, M., LeFew, W. R. et al. (2014). Multi-well microelectrode array recordings detect neuroactivity of ToxCast compounds. Neurotoxicology 44, 204-217. https://doi.org/10.1016/j. neuro.2014.06.012.

Vassallo, A., Chiappalone, M., De Camargos Lopes, R. et al. (2017). A multi-laboratory evaluation of microelectrode array-based measurements of neural network activity for acute neurotoxicity testing. Neurotoxicology 60, 280-292. https://doi.org/10.1016/j.neuro.2016.03.019.

Vatine, G. D., Barrile, R., Workman, M. J. et al. (2019). Human iPSC-Derived Blood-Brain Barrier Chips Enable Disease Modeling and Personalized Medicine Applications. Cell Stem Cell 24, 9951005.e6. https://doi.org/10.1016/j.stem.2019.05.011. 
Volpato, V., Smith, J., Sandor, C. et al. (2018). Reproducibility of Molecular Phenotypes after LongTerm Differentiation to Human iPSC-Derived Neurons: A Multi-Site Omics Study. Stem Cell Reports 11, 897-911. https://doi.org/10.1016/j.stemcr.2018.08.013.

Wagner, C. A., Friedrich, B., Setiawan, I. et al. (2000). The use of Xenopus laevis oocytes for the functional characterization of heterologously expressed membrane proteins. Cell Physiol Biochem 10, 1-12. https://doi.org/10.1159/000016341.

Wakeman, D. R., Hiller, B. M., Marmion, D. J. et al. (2017). Cryopreservation Maintains Functionality of Human iPSC Dopamine Neurons and Rescues Parkinsonian Phenotypes In Vivo. Stem Cell Reports 9, 149-161. https://doi.org/10.1016/j.stemcr.2017.04.033.

Wallace, K., Strickland, J. D., Valdivia, P. et al. (2015). A multiplexed assay for determination of neurotoxicant effects on spontaneous network activity and viability from microelectrode arrays. Neurotoxicology 49, 79-85. https://doi.org/10.1016/j.neuro.2015.05.007.

Walz, W. (2000). Role of astrocytes in the clearance of excess extracellular potassium. In Neurochemistry International (291-300). https://doi.org/10.1016/S0197-0186(99)00137-0.

Wang, C., Nie, X., Zhang, Y. et al. (2015). Reactive oxygen species mediate nitric oxide production through ERK/JNK MAPK signaling in HAPI microglia after PFOS exposure. Toxicol Appl Pharmacol 288, 143-151. https://doi.org/10.1016/j.taap.2015.06.012.

Wang, J., Pan, Y., Cui, Q. et al. (2018). Penetration of PFASs Across the Blood Cerebrospinal Fluid Barrier and Its Determinants in Humans. Environ Sci Technol 52, 13553-13561. https://doi. org/10.1021/acs.est.8bo4550.

Wang, Y., Liu, W., Zhang, Q. et al. (2015). Effects of developmental perfluorooctane sulfonate exposure on spatial learning and memory ability of rats and mechanism associated with synaptic plasticity. Food Chem Toxicol 76, 70-76. https://doi.org/10.1016/j.fct.2014.12.008.

Ward, L. M. (2003). Synchronous neural oscillations and cognitive processes. Trends Cogn Sci 7, 553-559. https://doi.org/10.1016/j.tics.2003.10.012.

Westerink, R. (2006). Targeting Exocytosis: Ins and Outs of the Modulation of Quantal Dopamine Release. CNS Neurol Disord - Drug Targets 5, 57-77. https://doi.org/10.2174/187152706784111597.

Westerink, R. H. S. (2013). Do we really want to REACH out to in vitro? Neurotoxicology 39, 169-172. https://doi.org/10.1016/j.neuro.2013.10.001.

Willmann, C. A., Hemeda, H., Pieper, L. A. et al. (2013). To Clone or Not to Clone? Induced Pluripotent Stem Cells Can Be Generated in Bulk Culture. PLoS One 8. https://doi.org/10.1371/ journal.pone.0065324.

World Health Organization (2013). WHO | Mental disorders affect one in four people. WHO. Available at: https://www.who.int/whr/2001/media_centre/press_release/en/ [Accessed October 22, 2019].

Wu, M. Sun, R., Wang, M. et al. (2017). Analysis of perfluorinated compounds in human serum from the general population in Shanghai by liquid chromatography-tandem mass spectrometry (LCMS/MS). Chemosphere 168, 100-105. https://doi.org/10.1016/J.CHEMOSPHERE.2016.09.161.

$\mathrm{Wu}, \mathrm{X}$., Yang, X., Majumder, A. et al. (2017). Astrocytes are protective against chlorpyrifos developmental neurotoxicity in human pluripotent stem cell-derived astrocyte-neuron cocultures. Toxicol Sci 157, 410-420. https://doi.org/10.1093/toxsci/kfxo56.

$\mathrm{Xu}, \mathrm{X}$., Lei, Y., Luo, J. et al. (2013). Prevention of $\beta$-amyloid induced toxicity in human iPS cellderived neurons by inhibition of Cyclin-dependent kinases and associated cell cycle events. Stem Cell Res 10, 213-27. https://doi.org/10.1016/j.scr.2012.11.005. 
Yang, J., Wang, C., Nie, X. et al. (2015). Perfluorooctane sulfonate mediates microglial activation and secretion of TNF- $\alpha$ through Ca2+-dependent PKC-NF-KB signaling. Int Immunopharmacol 28, 52-60. https://doi.org/10.1016/j.intimp.2015.05.019.

Ylä-Outinen, L. (2010). Human cell-based micro electrode array platform for studying neurotoxicity. Front Neuroeng 3. https://doi.org/10.3389/fneng.2010.00111.

Yuan, Y. and Atchison, W. D. (2007). Methylmercury-induced increase of intracellular Ca2+ increases spontaneous synaptic current frequency in rat cerebellar slices. Mol Pharmacol 71, 1109-1121. https://doi.org/10.1124/mol.106.031286.both.

Yuan, Y., Otero-Montañez, J. K. L., Yao, A. et al. (2005). Inwardly rectifying and voltage-gated outward potassium channels exhibit low sensitivity to methylmercury. Neurotoxicology 26, 439-454. https://doi.org/10.1016/j.neuro.2005.03.005.

Zeng, H., Guo, M., Martins-Taylor, K. et al. (2010). Specification of region-specific neurons including forebrain glutamatergic neurons from human induced pluripotent stem cells. PLoS One 5. https://doi.org/10.1371/journal.pone.0011853.

Zhang, L., Li, Y., Zeng, H. et al. (2011). Perfluorooctane Sulfonate Induces Apoptosis in N9 Microglial Cell Line. Int J Toxicol 30, 207-215. https://doi.org/10.1177/1091581810387832.

Zhang, N., An, M. C., Montoro, D. et al. (2010). Characterization of human Huntington's disease cell model from induced pluripotent stem cells. PLoS Curr, 1-11. https://doi.org/10.1371/ currents. RRN1193.

Zhang, Q., Liu, W., Zhao, H. et al. (2019). Developmental perfluorooctane sulfonate exposure inhibits long-term potentiation by affecting AMPA receptor trafficking. Toxicology 412, 55-62. https://doi.org/10.1016/J.TOX.2018.11.015.

Zimmerman, T. J. (1981). 4. Pilocarpine. Ophthalmology 88, 85-88. https://doi.org/10.1016/So161$6420(81) 35072-6$.

Zwartsen, A., Hondebrink, L. and Westerink, R. H. (2019). Changes in neuronal activity in rat primary cortical cultures induced by illicit drugs and new psychoactive substances (NPS) following prolonged exposure and washout to mimic human exposure scenarios. Neurotoxicology 74, 28-39. https://doi.org/10.1016/J.NEURO.2019.05.004.

Zwartsen, A., Hondebrink, L. and Westerink, R. H. (2018). Neurotoxicity screening of new psychoactive substances (NPS): Effects on neuronal activity in rat cortical cultures using microelectrode arrays (MEA). Neurotoxicology 66, 87-97. https://doi.org/10.1016/j. neuro.2018.03.007. 
NEDERLANDSE SAMENVATTING 


\section{NEDERLANDSE SAMENVATTING}

\section{ONTWIKKELING VAN GENEESMIDDELEN}

Wereldwijd stijgt het aantal mensen dat de komende jaren wordt gediagnosticeerd met een psychische of neurodegeneratieve aandoening. Volgens een schatting van de Wereldgezondheidsorganisatie (WHO) lijdt 1 op de 4 mensen aan een psychische aandoening. Psychische en neurodegeneratieve aandoeningen hebben een grote impact op het dagelijks bestaan van patiënten en hun naasten. Medicijnen kunnen het lijden verlichten. De combinatie van de groeiende groep patiënten en de hoge ziektelast leidt tot een verhoogde vraag naar geneesmiddelen die effect hebben op het brein.

Het ontwikkelen van geneesmiddelen is een langdurig proces dat tot wel 15 jaar in beslag kan nemen (zie hoofdstuk 1, Fig. 1). Het proces begint met 1000 stofjes die potentieel zouden kunnen werken, 5 daarvan komen in klinische trials terecht en uiteindelijk komt er 1 als effectief geneesmiddel op de markt. Dit geneesmiddel moet wel aan alle veiligheidseisen voldoen. Zo mag het nieuwe middel geen negatieve effecten op het zenuwstelsel en dus het brein hebben. Met andere woorden, het middel mag niet neurotoxisch zijn. Bij mensen kan neurotoxiciteit zich uiten als veranderingen in gedrag, geheugen, capaciteit om te leren en in veranderingen in beweging. Ook mag het middel geen gevaarlijke (neurotoxische) bijwerkingen hebben. De meest gevaarlijke, en soms fatale, bijwerking op het brein dat een geneesmiddel kan hebben is het veroorzaken van een (epileptische) aanval. Tijdens zo'n aanval, ook wel insult genoemd, is de activiteit van het zenuwstelsel, de neuronale activiteit, sterk verhoogd. Doordat de zenuwcellen, neuronen genaamd, tegelijk actief worden veranderd het patroon van de activiteit. De activiteit is meer gesynchroniseerd. Het gevolg is dat het brein gedurende de tijd die een aanval duurt niet goed functioneert.

\section{VEILIGHEIDSONDERZOEK}

Geneesmiddelen die op het brein werken, geven een verhoogd risico op het veroorzaken van een insult. Dit komt omdat ze de bloed-hersen barrière, een grens die het brein beschermt, over moeten om hun werking te kunnen uitvoeren. Er moet dus onderzoek worden gedaan naar de potentie van een middel om neurotoxiciteit of een insult te kunnen veroorzaken. Voor dit onderzoek worden proefdieren, vaak ratten en muizen, gebruikt. Dat zijn in vivo studies. De studies worden vaak pas laat in het geneesmiddelontwikkelingsproces gedaan. Het 
gevolg is, dat wanneer dan pas ontdekt wordt dat een middel een insult kan veroorzaken of neurotoxisch is, er al veel tijd en proefdieren zijn geïnvesteerd.

Hoewel een insult een zeer ernstige bijwerking is, zijn er geen duidelijke richtlijnen over hoe het onderzoek naar de potentie om een insult te veroorzaken gedaan moet worden. Naast de in vivo studies, is er een aantal in vitro (experimenten die gebruik maken van celsystemen) en ex vivo (experimenten waarin weefsel of cellen van dieren worden gebruikt) technieken die gebruikt kunnen worden om het optreden van een insult op te sporen. Deze technieken zijn soms succesvol in het detecteren van een insult, maar ze gebruiken nog steeds proefdieren. Naast het feit dat proefdiergebruik ethisch discutabel, tijdrovend en duur is, brengt het nog een uitdaging mee. De uitkomsten van studies gedaan op proefdieren, meestal knaagdieren, moeten worden vertaald naar risico's voor de mens. Helaas zijn knaagdieren geen mini-mensen en kan deze vertaalslag niet zomaar worden gemaakt. Het is bekend dat het brein van knaagdieren zich anders ontwikkelt dan het brein van mensen. Daarnaast is bekend dat de neuronen, zenuwcellen, van knaagdieren zich anders gedragen dan neuronen van mensen. Dat het vertalen van resultaten van knaagdieren naar mensen lastig is, reflecteert zich in het feit dat ondanks de vele onderzoeken naar veiligheid er nog steeds geneesmiddel van de markt worden gehaald die toch een insult blijken te kunnen veroorzaken. Dit geeft ook aan dat de huidige modellen om deze insulten mee op te sporen, niet voldoende voorspellend zijn. Het is dus duidelijk dat er behoefte is aan een nieuw model. Bij voorkeur maakt dit model gebruik van menselijke (humane) cellen zodat de vertaling van de resultaten makkelijker wordt en er minder proefdieren nodig zijn.

\section{ALTERNATIEVEN VOOR PROEFDIEREN}

Bezorgdheid over de bruikbaarheid van de resultaten van proefdieronderzoek en het gebruik van proefdieren zelf heeft ertoe geleid dat er veel onderzoek is gedaan naar de ontwikkeling van alternatieven. Een doorbraak was de ontdekking dat fibroblasten zo kunnen worden geprogrammeerd dat ze ieder ander celtype kunnen aannemen. Fibroblasten zijn bindweefselcellen die onder andere in de huid van mensen voorkomen en dus makkelijk beschikbaar zijn. Deze ontdekking resulteerde in de introductie van menselijke/humane, geïnduceerde pluripotente stamcellen (hiPSCs). Deze cellen kunnen weer geprogrammeerd worden tot neuronen (hiPSC-neuronen zie hoofdstuk 1, Fig. 2) en astrocyten (ondersteunde cellen; hiPSC-astrocyten). Het ontwikkelen van deze hiPSC-neuronen en hiPSCastrocyten is erg tijdrovend, maar tegenwoordig zijn deze cellen ook commercieel 
te verkrijgen. Daarmee vormen ze een interessante optie voor neurotoxicologisch onderzoek. Echter, een nadeel van deze cellen is dat er nog weinig over bekend is.

\section{DOEL VAN DIT PROEFSCHRIFT}

Humane iPSC-neuronen en -astrocyten kunnen een alternatief zijn voor proefdiergebruik, maar er is nog weinig over deze cellen bekend. Het doel van het onderzoek dat in dit proefschrift wordt beschreven, is om een in vitro celmodel met hiPSC-neuronen en -astrocyten te ontwikkelen dat kan worden gebruikt voor neurotoxicologisch onderzoek. Speciale aandacht gaat uit naar de mogelijkheid van het model om een insult te kunnen detecteren. Om te onderzoeken of het ontwikkelde hiPSC-model hiervoor geschikt is, staan in de verschillende hoofdstukken steeds de volgende vier vragen centraal.

- Ontwikkelt het hiPSC-model neuronale netwerken die lijken op de netwerken in ons brein?

- Vertonen deze netwerken neuronale activiteit?

- Kan het model een aanval detecteren?

- Hoe verhoudt dit model zich tot de huidige gouden standaard, een ex vivo rat brein model?

\section{NEURONALE ACTIVITEIT EN HET OPSPOREN VAN EEN AANVAL}

Een van de eerste stappen is dus om te onderzoeken of de hiPSC-cellen eigenschappen vertonen die vergelijkbaar zijn met het menselijke brein. De belangrijkste functie van het zenuwstelsel, met het brein als centrum, is het ontvangen, verzenden en interpreteren van signalen. Dit heet ook wel neurontransmissie of neuronale communicatie. Het hele systeem bestaat uit miljoenen neuronen en ondersteunende cellen. Neuronen zijn speciaal uitgerust voor neurotransmissie. Aan de ene kant van hun cellichaam hebben ze dendrieten om signalen mee te ontvangen, en aan de andere kant een axon om signalen mee te versturen (zie hoofdstuk 1, Fig. 3 links). Om het signaal van de ene cel naar de andere cel over te brengen worden signaleringsmoleculen, neurotransmitters gebruikt. Afhankelijk van het type neurotransmitter wordt de ontvangende cel geactiveerd of geremd. De belangrijkste activerende neurotransmitter is glutamaat. GABA is de meest voorkomende remmende neurotransmitter. Neuronen die glutamaat afgeven heten glutamatarge neuronen en neuronen die GABA afgeven GABA-erge neuronen. De ontvangende cel vangt het signaal op via de bijbehorende neurotransmitter receptor. Voor het goed functioneren van neurotransmissie moet er een goede balans zijn tussen stimulerende en remmende neurotransmitters. Een verstoring in deze balans kan een insult veroorzaken. 
Er zijn verschillende technieken om te onderzoeken of cellen (functionele) netwerken vormen. In de eerste instantie is het belangrijk om te weten of er een netwerk gevormd is. Zoals beschreven staat in hoofdstuk 3, kunnen de netwerken worden gevisualiseerd door bepaalde specifieke delen van cellen een fluorescent label te geven en kan vraag 1 worden beantwoord. Aanwezigheid van de benodigde neurotransmitters kan worden bevestigd met calcium imaging (hoofdstuk 5), maar deze techniek kan ook worden gebruikt om te onderzoeken of een netwerk functioneel is (hoofdstuk 3), en dus om vraag 2 te beantwoorden. Vraag 2 kan ook worden beantwoord door gebruik te maken van een meer high-throughput techniek, namelijk de micro-elektrode array (MEA). Voor deze techniek worden neuronale netwerken over elektrodes gegroeid zoals beschreven staat in hoofdstuk 3. Deze elektrodes kunnen de activiteit van een netwerk meten. Aangezien met deze techniek ook veranderingen kunnen worden gemeten na toediening van toxische stoffen of geneesmiddelen, kan het worden gebruikt om een insult mee te detecteren (hoofdstuk 4-8). MEA helpt dus ook bij het beantwoorden van vraag 3. Omdat er verschillende soorten cellen op kunnen worden gekweekt, kan er dus een vergelijking tussen humane en rat cellen worden gemaakt en helpt de techniek bij het beantwoorden van vraag 4 (hoofdstuk 4,7 en 8). Deze techniek vormt een groot contrast met de meer traditionele voltage-clamp techniek waar naar een enkele receptor wordt gekeken (hoofdstuk 2 en 4).

\section{TOEPASBAARHEID VAN HIPSC-NEURONEN EN -ASTROCYTEN}

In hoofdstuk 5 is te zien dat mono-culturen met alleen hiPSC-neuronen complexe, actieve neuronale netwerken vormen met remmende en activerende neuronen. Ook zijn de benodigde neurotransmitter receptoren aanwezig. Ondanks het feit dat deze netwerken actief zijn, is de activiteit veel lager dan in het rat cortex celmodel. Hoewel de activiteit laag is, kan het netwerk wel gemoduleerd worden met de neurotransmitters glutamaat en GABA en met de neurotoxicologische stoffen endosulfan en amfetamine op een manier die vergelijkbaar is met het rat model.

Het is bekend dat de netwerkactiviteit verhoogd kan worden door toevoeging van astrocyten aan het netwerk. Ook kunnen de astrocyten de gevoeligheid van het netwerk voor stoffen beïnvloeden. In hoofdstuk 6 zijn er astrocyten aan de netwerken toegevoegd en werden zogenaamde co-culturen gemaakt. Daarnaast zijn verschillende verhoudingen glutamatarge tot GABA-erge neuronen getest. De verschillende modellen vormden allemaal complexe netwerken. Ook werden alle netwerken actief, waarbij de neuronale activiteit in de co-culturen hoger was dan in mono-culturen. In de co-cultuur met extra GABA-erge neuronen was de activiteit 
wat lager dan in de co-cultuur zonder GABA-erge neuronen. Daarnaast was het patroon van de activiteit beter gesynchroniseerd in deze modellen. De activiteit van de co-culturen was vergelijkbaar met het rat cortex model. Omdat het model insulten moet kunnen detecteren, werden de co-culturen blootgesteld aan stoffen die een insult kunnen veroorzaken: picrotoxine (PTX) en endosulfan. Wat blijkt is dat de co-cultuur met extra GABA-erge neuronen gevoeliger is voor deze stoffen dan de co-cultuur zonder extra GABA-erge neuronen. Uit bovenstaande blijkt dus dat astrocyten belangrijk zijn voor de ontwikkeling van gesynchroniseerde netwerkactiviteit en dat GABA-erge neuronen belangrijk zijn bij het detecteren van een insult.

Voor het onderzoek in hoofdstuk 7 hebben wij van drie verschillende leveranciers hiPSC-neuronen en -astrocyten verkregen. Deze drie modellen verschillen in de hoeveelheid cellen die worden uitgeplaat op de elektrodes van de MEA, de ratio tussen astrocyten en neuronen, en de verhouding glutamatarge en GABA-erge neuronen. Alle drie de modellen ontwikkelden complexe netwerken die op de in vivo brein structuur lijken. Ook vertoonden alle drie de modellen spontane netwerkactiviteit. Het model met de hoogste verhouding astrocyten, ontwikkelde de hoogste activiteit. Hoewel er verschillen tussen de modellen zat, is de ontwikkeling vergelijkbaar met het rat cortex model. Om uit te vinden of de modellen geschikt zijn voor het detecteren van insulten, zijn ze blootgesteld aan PTX, 4-aminopyridine (4-AP) en strychnine. Al deze stoffen kunnen een insult veroorzaken. Om een vergelijking te maken met het rat cortex model, is dit model ook gekweekt en blootgesteld. De verkregen data laat zien dat alle modellen een insult kunnen detecteren op een manier vergelijkbaar of beter dan het rat cortex model.

Het hiPSC-model met de kortste kweektijd en een hoge activiteit werd geselecteerd voor een uitgebreider onderzoek. Dit model werd blootgesteld aan negen extra stoffen (pentylenetetrazole (PTZ), amoxapine, enoxacine, amoxicilline, pilocarpine, linopirdine chloorpromazine (CPZ), fenytoïne en paracetamol). Van deze stoffen is bekend of ze wel of geen insult kunnen veroorzaken. Weer werd ter vergelijking het rat cortex model in parallel blootgesteld. Uit de data blijkt dat beide modellen (rat en humaan) reageren op de blootstelling, maar niet alle stoffen werden in beide modellen even goed opgepikt. PTZ en enoxacin waren geen hit in het hiPSC model. In het rat model konden amoxicilline en pilocarpine niet worden gedetecteerd. Voor sommige stoffen was de gevoeligheid van het model ook anders. Het hiPSC model was gevoeliger voor linopirdine en het rat model voor fenytoïne. Deze studie laat dus zien dat hiPSC modellen op een grotendeels vergelijkbare manier reageren als 
de gouden standaard, het rat cortex model. Net als hoofdstuk 7 laat dit hoofdstuk zien dat er desondanks verschillen zijn tussen het humane en rat model. We laten met dit onderzoek dus zien dat het humane model al wel kan worden gebruikt voor het opsporen van insulten, maar dat de gouden standaard nu nog niet volledig kan worden vervangen.

\section{CONCLUSIES}

Het doel van dit proefschrift was om een hiPSC-model te ontwikkelen dat kan worden gebruikt voor neurotoxicologisch onderzoek en het detecteren van insulten. Gedurende het onderzoek is steeds getracht om antwoord te vinden op vier vragen gerelateerd aan netwerkformatie en -activiteit, het detecteren van een insult en de vergelijking met de huidige gouden standaard. De data gepresenteerd in dit proefschrift laat zien dat hiPSC-neuronen en -astrocyten complexe netwerken vormen en spontane netwerkactiviteit ontwikkelen. Ook hebben we laten zien dat de verhouding tussen astrocyten en neuronen en tussen glutamatarge en GABAerge neuronen een belangrijke rol speelt voor de activiteit én voor de gevoeligheid van de netwerken. Hiermee beantwoorden we dus vraag 1 en 2 . Ook hebben we laten zien dat de hiPSC modellen kunnen worden gebruikt om insulten te detecteren en geven we dus een antwoord op vraag 3 . Het beantwoorden van vraag 4 is moeilijker, want er zijn verschillen tussen het humane en rat model. Dit is waarschijnlijk het logische gevolg van de verschillen tussen mensen en ratten. De vraag is dus, welk model voorspelt het risico op een insult bij mensen beter? Deze vraag moet worden beantwoord voordat een goed antwoord op vraag 4 kan worden gegeven. Ook moet deze vraag worden beantwoord voordat humane modellen het rat model volledig kunnen vervangen en proefdieren niet meer nodig zijn het testen van neurotoxiciteit. Wel heeft het onderzoek in dit proefschrift al laten zien dat hiPSC modellen een belangrijke verschuiving in de manier van onderzoek kunnen veroorzaken richting proefdiervrij onderzoek. 
ACKNOWLEDGEMENTS 


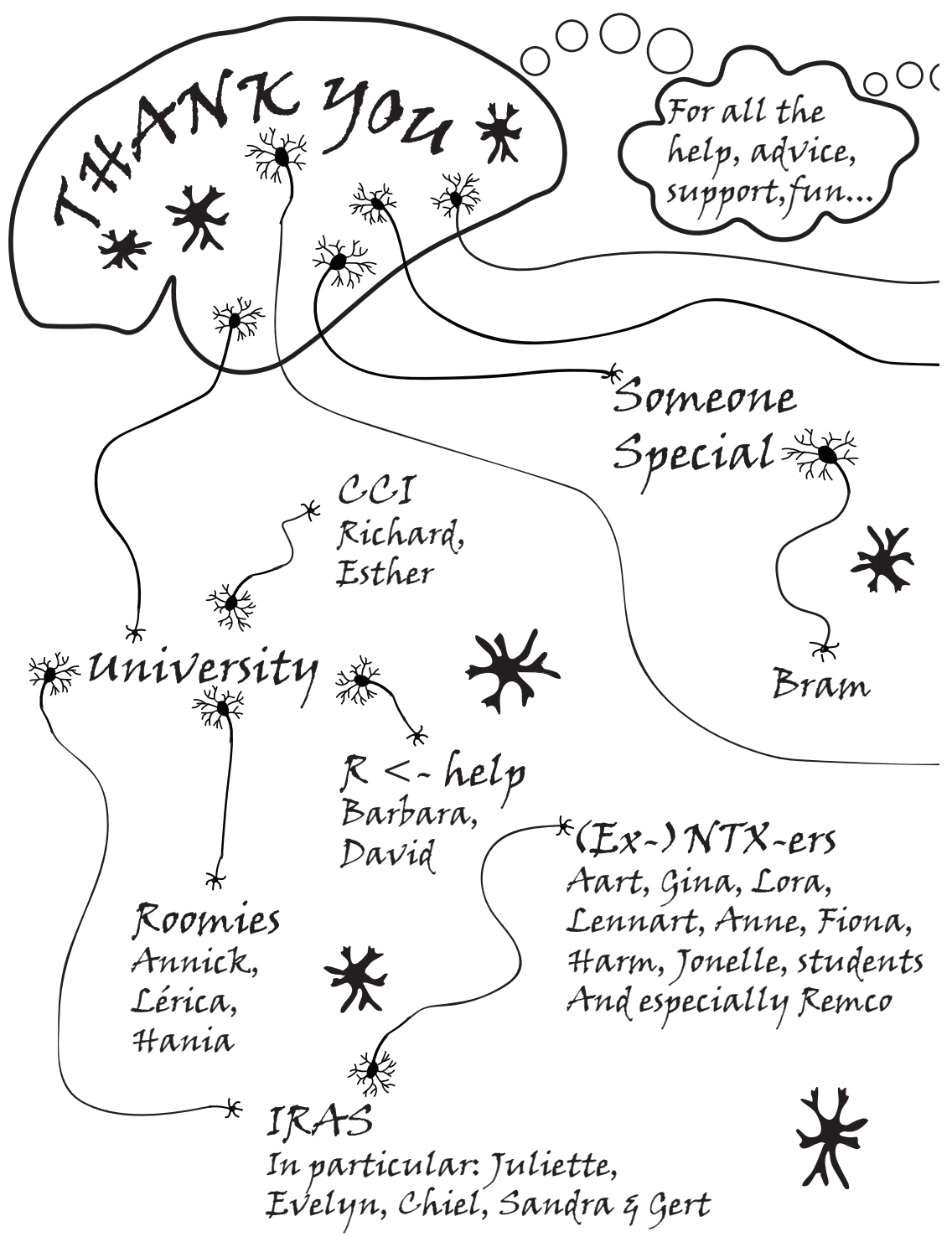



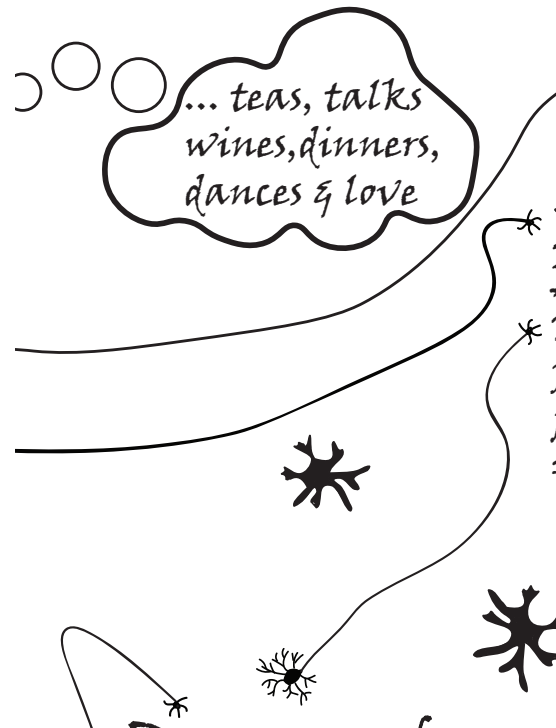
Paranymphs

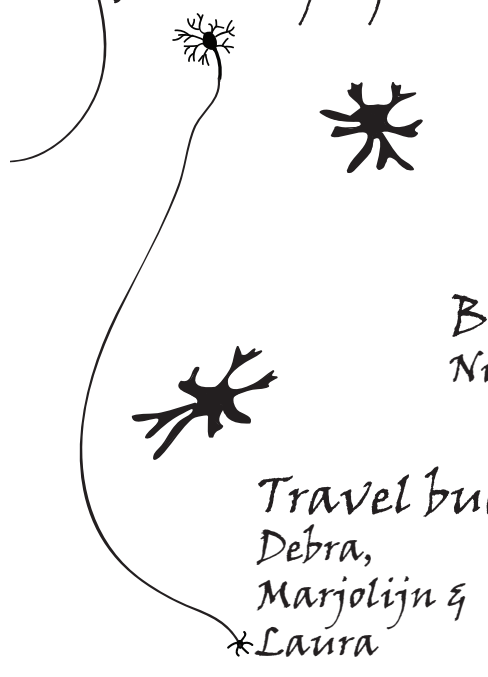

Blues-bug Nico

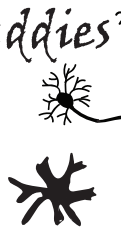

Family

Especially: Pap \&Mam, * Niels, Esther \& Livia

Peter $\overline{\text { Jeanine, }}$ Rolf, Lidi, René, Hanneke, Harmen 5 Geert

Friends

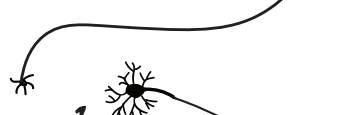

Eveline, Casper,

Darren, Kamil,

Carolina, Jort,

Hugo, Lisette,

Steven 5 Michelle
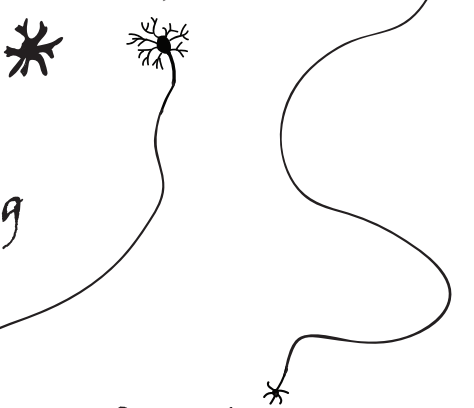

MDWAP-gang

* Rianne, Victoria 5 Melinda 


\section{ABOUT THE AUTHOR}

\section{Anke Tukker}

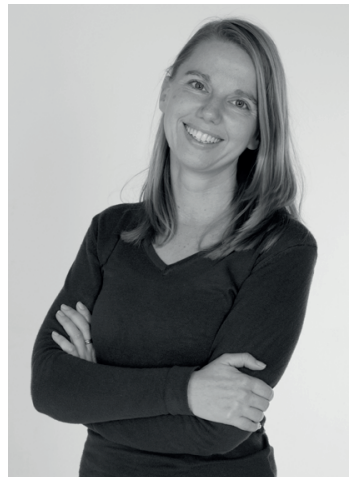

Anke Tukker was born on November $9^{\text {th }} 1986$ in Almere, where she also grew up. After graduating from secondary school at the Goois Lyceum (Bussum, The Netherlands), she started her study Biomedical Sciences at Utrecht University (Utrecht, The Netherlands). Anke obtained her bachelor of Science degree in 2010 and spend the next year teaching and tutoring biology and chemistry at PIOS (Bussum/ Hilversum, The Netherlands). Following this year, she started a master's programme in Latin American studies at the University of Amsterdam (Amsterdam, the Netherlands) for which she conducted four months of field work at a coffee plantation in Nicaragua. At this plantation, she investigated how employees perceived their health in relation to the work they did. Upon obtaining her master of Arts degree (2013), Anke went back to Utrecht University and continued her education with a master of Science in Toxicology and Environmental Health. During this programme she performed an internship at the Institute for Biodiversity and Ecosystem Dynamics (IBED, University of Amsterdam, Amsterdam, The Netherlands) and at the Neurotoxicology Research group at IRAS (Utrecht, The Netherlands). After completion of this master (2015), Anke worked as a regulatory affairs officer at VSM (Alkmaar, The Netherlands) and medical information officer at MSD (Wormerveer, The Netherlands) before starting her PhD. From January 2016 until March 2020, she worked as a PhD student under supervision of Dr. Remco H.S. Westerink and Prof. dr. ir. Juliette Legler on the research presented in this thesis. This research was part of the CRACK-it Neuratect challenge. During her PhD she successfully followed several postgraduate courses of the postgraduate education in toxicology (PET) programme to obtain her registration as a European Registered Toxicologist (ERT). She also helped to develop and coordinate the Neurotoxicology course for this programme. Anke will continue her academic career as a post-doctoral researcher at Purdue University (West Lafayette, IN, USA) in the research group of Prof. dr. Aaron B. Bowman. This group focusses on the role of gene - environment interactions between metal exposure and neurodegenerative diseases. 


\section{LIST OF PUBLICATIONS, AWARDS AND CONFERENCE ORAL PRESENTATIONS}

\section{List of publications}

Tukker AM, de Groot MWGDM, Wijnolts FMJ, Kasteel EEJ, Hondebrink L, Westerink RHS (2016), Is the time right for in vitro neurotoxicity testing using human iPSC-derived neurons?, ALTEX, 33 (3) 261-271, DOI: 10.14573/ altex.1510091

Hondebrink L, Kasteel EEJ, Tukker AM, Wijnolts FMJ, Verboven AHA, Westerink RHS (2017), Neuropharmacological characterization of the new psychoactive substance methoxetamine. Neuropharmacology, 123 pp. 1-9, DOI: 10.1016/j. neuropharm.2017.04.035

Hammer J, Tukker AM, Postma JF, Haftka JJ-H, Hermens JLM, de Voogt P, Kraak MHS (2018). Solubility constraints on aquatic ecotoxicity testing of anionic surfactants. Bulletin of Environmental Contamination and Toxicology, 101, pp. 99-104, DOI: 10.1007/s00128-018-2361-1

Tukker AM, Wijnolts FMJ, de Groot A, Westerink RHS (2018), Human iPSC-derived neuronal models for in vitro neurotoxicity assessment, Neurotoxicology, 67, pp. 215-225, DOI: 10.1016/j.neuro.2018.06.007

Tukker AM, Wijnolts FMJ, Groot de A, Wubbolts RW and Westerink RHS. In vitro techniques for assessing neurotoxicity using human iPSC-derived neuronal models. In: Aschner M, Costa L (eds). Neuromethods. Springer; 2019. Pp. 17-35. DOI: $10.1007 / 978-1-4939-9228-7 \_2$

Tukker AM, van Kleef RGDM, Wijnolts FMJ, de Groot A, Westerink RHS (2020), Towards animal-free neurotoxicity screening: Applicability of hiPSC-derived neuronal models for in vitro seizure liability assessment. ALTEX, 37, pp. 121-135 DOI: $10.14573 /$ altex.1907121

Tukker, A. M., Bouwman, L. M. S., van Kleef, R. G. D. M. et al. (2020). Perfluorooctane sulfonate (PFOS) and perfluorooctanoate (PFOA) acutely affect human $\alpha 1 \beta 2 \gamma_{2} \mathrm{~L}$ GABAA receptor and spontaneous neuronal network function in vitro. Sci Rep 10. DOI: 10.1038/s41598-020-62152-2. 


\section{Awards}

Speed presentation PhD award, $1^{\text {st }}$ place, at the annual meeting of the Netherlands Society for Toxicology (Nederlandse Vereniging voor Toxicologie [NVT]), June 2016, Soesterberg, The Netherlands

David Ray Student Travel Award for best oral presentation at the bi-annual meeting of the International Neurotoxicology Association (INA), May 2017, Florianopolis, Brazil

INA-NTSS travel award at the bi-annual meeting of the INA, May 2017, Florianopolis, Brazil

NVT travel award, October 2019, The Netherlands

Nominated for the Hugo van Poelgeest Award (top three, winner announced on October $2^{\text {nd }}, 2020$ ), December 2019, The Netherlands

\section{Conference oral presentations}

Human iPSC-derived neurons on micro-electrode arrays (MEAs) for in vitro neurotoxicity testing. Annual meeting of the NVT, Soesterberg, The Netherlands (June 2016)

Applications of human induced pluripotent stem cell (3D) models for neurotoxicity testing. Bi-annual meeting of the INA, Florianopolis, Brazil, The Netherlands (May 2017), invited speaker

PFOS and PFOA: What do they do to your brain? Society of Toxicology (SOT) meeting, Baltimore, MD, USA (March 2019)

Towards animal free in vitro neurotoxicity testing: Are we there yet? Bi-annual meeting of the INA, Düsseldorf, Germany (October 2019), invited speaker and session chair 
